\title{
Worn Out or Worn In?
}



Ross Ernest Stevens

2006

This book is submitted as a commentary on Design Research by Composition undertaken for the Degree of Master of Design Victoria University of Wellington. 
Table of Contents

\section{Acknowledgements}

\section{Abstract}

1 Introduction

2. Products to Artifacts

2.1 Weathering and Wear

2.2 The Importance of Artifacts

2.3 The Future and Industrial Design

2.4 Designers as Co-authors

2.5 Designed Evolution

2.6 People as Creators

2.7 Personalising Products

2.8 Softwear Design

2.9 Physical Limitations

2.10 Customising Through Use

2.11 Associations in Products

2.12 Automous Skins

2.13 Generic Commodities

2.14 Conclusion

3. Manufacturing Artifacts

3.1 Visual Diversity

3.2 Conforming Technology

3.3 Manufacturing Multiples

3.4 Reactive Metals

3.4 Process and Form Interaction

3.5 Process and Form
3.6 Unique Materials

$\begin{array}{ll}3.6 & \text { Unique Materials } \\ 3.7 & \text { Beyond Form Constraints }\end{array}$

3.7 Beyond Form Con
3.8 Creation History

3.9 Rapid Prototyping

3.10 Digital Uncertainty

3.11 Wear Revealing Detail

3.12 External and Internal Complexity

3.13 The Sensitivity of Data

3.14 The Sensitivy of Data

3.14 Virtual and Physical Sites
3.15 Re-construction Through Wear

3.16 Conclusion p1

p 5 


\section{Envisaging the Future}

4.1 Physical and Digital Simulation

4.2 Informed Consent

4.3 Connecting People

4.4 Informed Consumption

4.5 Conclusion

\section{Design Experiments}

5.1 Time and Design

5.2 Displaying Wear

5.3 Wear Stages

5.4 Finger Wear

5.5 Interface Wear

5.6 Multiple Layers

5.7 Multiple Messages

5.8 Interface Wear 3D

5.9 Wear Bias

5.10 From $2 \mathrm{D}$ to $3 \mathrm{D}$

5.10 From $2 D$ to

5.11 Growing $3 \mathrm{D}$
5.12 Visual Variation

5.12 Visual Variation
5.13 Surface Wear

5.13 Surface Wear
5.14 Enclosure Wear

5.14 Enclosure Wea
5.15 Applied Design

6 Conclusion

Appendix: Site Observations

p 263

Bibliography

p 319

Animations CD 



\section{Acknowledgements}

This research about the importance of time could not have happened without the generous gift of time by the following people.

Maxe Fisher, Supervisor

Bernard Guy

Edgar Rodriguez

Luke Feast

Michael Patterson

Richard Hartle

Russel Lowe

Vladimir Mako

Mark Elmore

Vien McArthur

Danny Coster

Gary Morrison

Lynley Hill

Petra Alsbach-Stevens 



\section{Abstract}

Worn out or worn in started with the creation of a building. For an industrial designer, this was unfamiliar territory. Through working with a specific site came the recognition of the potential of weathering to add a unique quality to the design that goes beyond its initial concept. The inclusion of this potential in the design required a projection into the future and an acceptance of the inevitability of influences that could not be fully controlled. Rain, sun, footprints and cobwebs would all add or subtract to the initially simplistic design concept. It was another realm of design: a 4 dimensional one.

Where does the equivalent of weathering exist within the familiar scale of mass produced products?

It is in the interaction between the body and the products through use.

The potential of this interaction to add another dimension to a design forms the core of this research. The body is a complex site: fluid, directed, precarious, yet nurturing. While it may at first seem unreasonable that the soft body could erode hard, seemingly durable materials, the evidence is al around us. Though it works at a time scale that is almost invisible to our everyday perception, over time the evidence is recorded in our products.

Through research by reading, observing, designing, making and unmaking products, the concept of designs embedded within products has emerged. Like a box of chocolates with a series of layers, this research addresses how the wearing away of one layer can reveal the existence of another. 



\section{Introduction}


As an experienced designer yet inexperienced academic researcher the temptation was to stay within the familiar, the realm of applied research. This seemed to undervalue the opportunity to undertake research that was not tied directly to the needs and limitations of industry. However, the idea of undertaking purely theoretical work seemed to overlook the design knowledge already obtained through first hand experience.

As such this research is sited between academic theory and industrial design practice. As a field of research it is largely unexplored with limited established research methods and precedent projects. From this situation grew an approach that borrowed from an older and more established field of research, namely science. The approach undertaken borrows a rigorous scientific methodology but also touches on concepts from pure science, applied science and science fiction.

These concepts have been transposed into the discipline of industrial design as pure design, applied design and design fiction.

The methods were adapted from science yet the primary area of investigation was the aesthetic qualities of products as they became worn. These qualities extend beyond ideas and definitions related to the visual qualities of new products that are isolated in time to aesthetic qualities that arise through the interaction between people and products over time. The evidence of a previous existence and the projections of a future existence collectively form the meaning associated with the product.

The scientific approach offered observation of the evidence left through interaction between people and existing products as the first point of entry into the research (see Appendix). As the designer jointly responsible for the twenty year old product on the opposite page I witnessed a design vision transform into a made, used and disposed of product. The evidence of disproportional wear in the product's buttons reveals a lack of understanding when the product was designed. The aim of this research is to develop greater understanding of the potential of time and use as mechanisms capable of creating desired and designed transformations in products throughout their lives. 


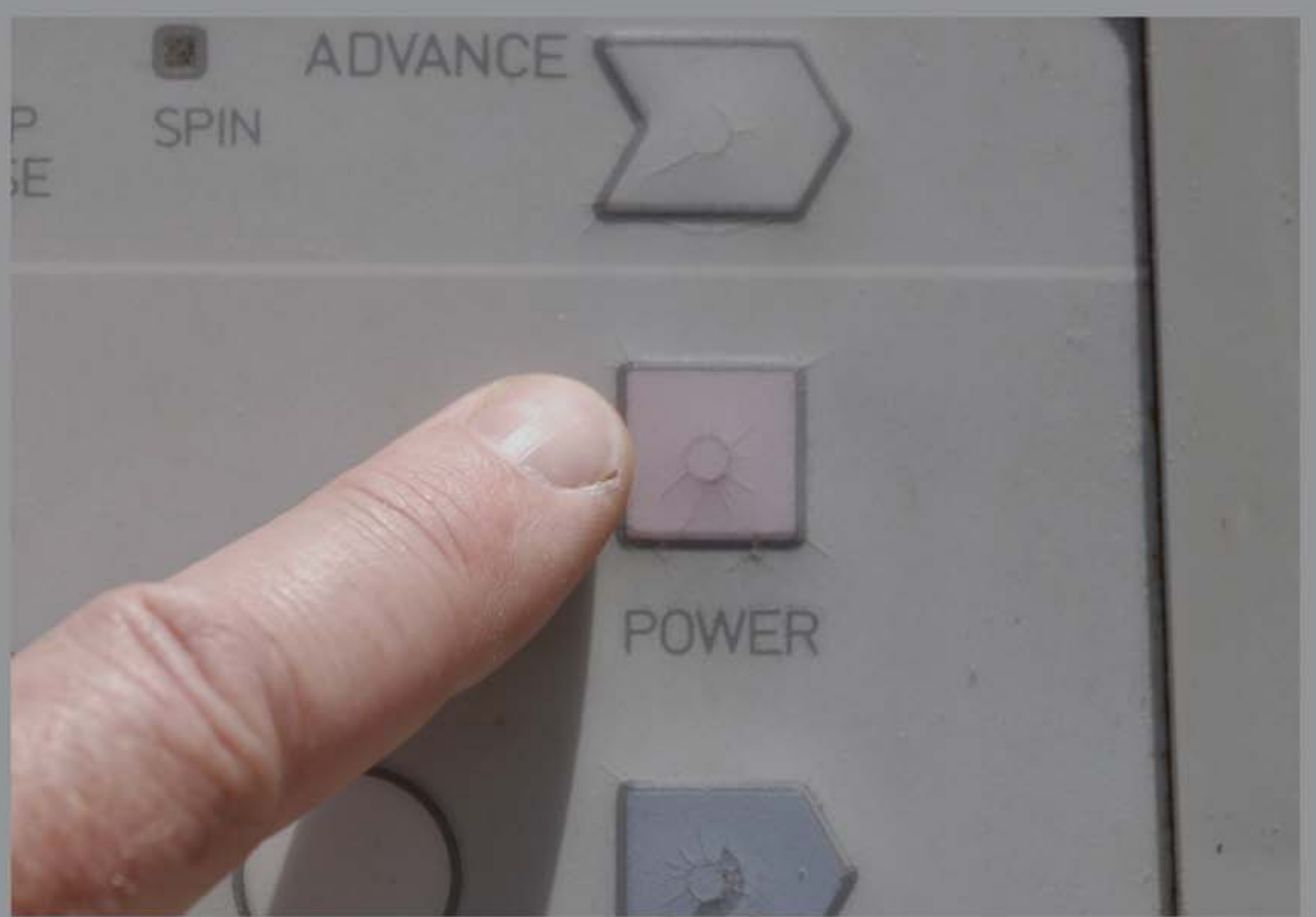

Clothes washing machine, Gentle Annie 1987 

2. Products to Artifacts 
This chapter explores the potential of human interaction to transform mass produced products into meaningful artifacts.

Finishing ends construction, weathering constructs finishes.

In the process of subtracting the "finish" of a construction, weathering adds the finish of the environment. Subtraction leads to final ruination and intimates, therefore, the end of the building as it would the death of a figure. Aging, then, can be seen as either benigh or tragic-or both. This raises

the question: beyond the general category of weathering as a romantic form of aging, are there other specific ways the unending process of deterioration can be understood, and then intended? Is it possible that weathering is not only a problem to be solved, or a fact to be neglected, but is an inevitable occurrence to be recognised and made use of in the uncertainties of its manifestations? 1

\subsection{Weathering and Wear}

Weathering as an inevitable occurrence to be recognised and made use of holds a parallel implication for wear in products capable of creating a site specific finish valued for the uncertainty of its manifestation.

The site observations in the appendix illustrate that the physical interaction between people and products is a viable mechanism to create a reactive site, capable of transforming products through time. Interaction between the hand and products created wear patterns that were

expressive of physical dexterity, intimate connection and extensive use. Portable electronic products, such as cell phones and music players were selected as the primary focus of this research because they offered a dynamic and varied site, relevant to emerging technology and to contemporary culture.

As static products they are inherently incapable of the movement required to create wear. Without mechanisms such as motors and wheels, they rely on a relationship with people to become dynamic. This dynamic movement creates interaction and friction that in turn wears away the surface of the product. These human powered wear patterns form evidence of a person's relationship with the physical world and tangible proof of the person's existence. By denying the environment the chance to add a site specific finish to a building an opportunity is lost. By denying people the right and ability to leave evidence created by wear in their products, the very meaning and value of a person's existence is put to question. 


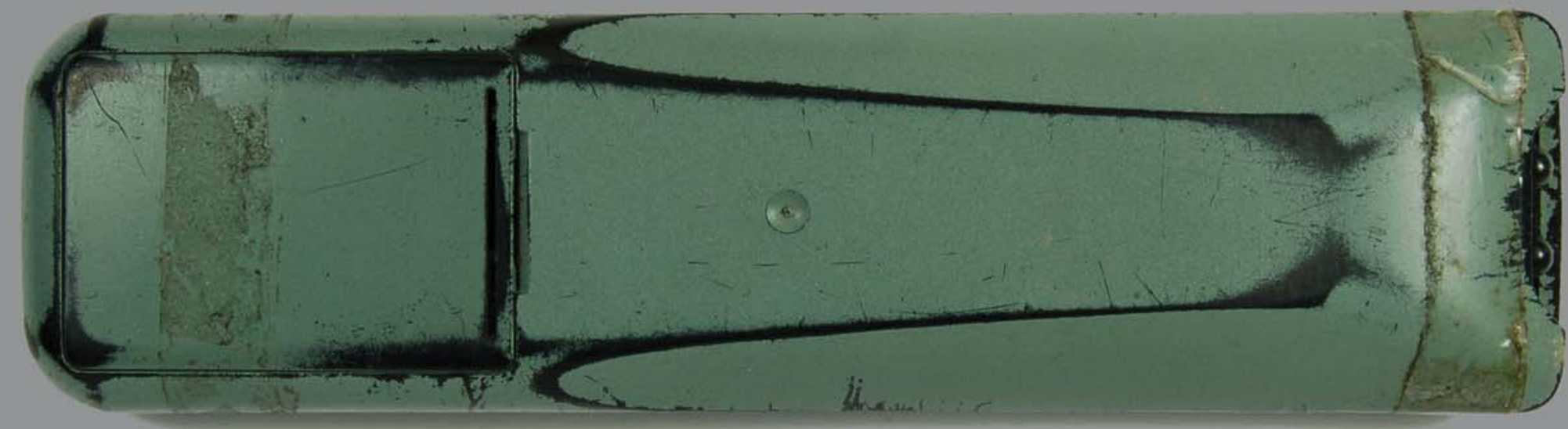

Television remote control. SABA 1993

The wear in this product was not designed, it was an unconsidered act. The contrasting colours of the base material and exterior highlight the subtleties and diversity of wear patterns. Abrupt scratches reveal instant force while the soft feathered edges reveal repetitive interactions. The wear occurs unevenly over the product's surface revealing previously imperceptible subtleties in the form. 


\subsection{Importance of Artifact}

Interpreting artifacts allows an understanding of the culture related to them, derived from evidence formed during the products creation or use

They can be communal artifacts that express qualities broadly related to groups of people or personal artifacts that express the relationship between a specific person and a specific product.

The nail punch on the opposite page is a personal artifact inherited from my grandfather. Without a direct memory connecting them, the associations and meaning derived from it are interpreted from the evidence left in its surface. The buildings and furniture that he created using the punch have become dispersed and are no longer accessible. They reveal his skill but the punch summarises of the amount of work undertaken throughout his life. When the punch was inherited it seemed unimportant until a personal interested in building emerged and then the true value of the artifact became apparent. It revealed to me that I had not only inherited the product but also the genetics of the person who had used it. It served as material evidence of invisible potential within me.

Artifacts collect evidence of the past that offers an understanding of the future.

Our memories give meaning to things, and at the same time the things exist as samples of a bygone experience. Their existence in daily life also creates new experiences, so it is not only the past they reflect, but the present as well. Things have both shared and individual meanings,

long-lasting as well as temporary. The memories associated with an object can give it a strong individual meaning. A souvenir, a remembrance, a memento is attached to a bygone encounter. The event is preserved in a tangible form of a keepsake. The special remembrance value may

disappear with its owner. On the other hand, it can remain as a reminder of its previous owner to relatives, or as a memento of an important person in a museum. Are not actually all museum artefacts reminiscences of a known or unknown user, maker or owner? 2 


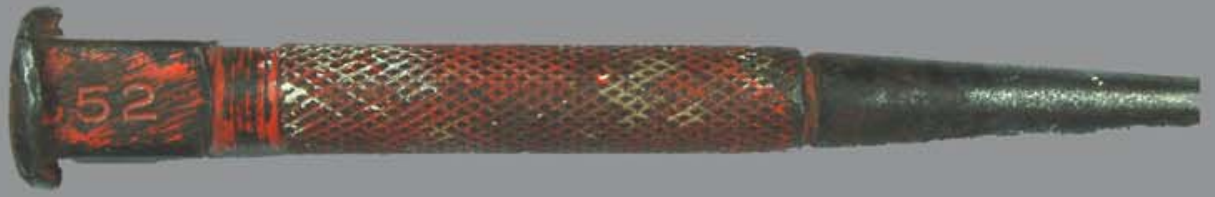

Worn nail punch. 1937 (estimate) 


\subsection{The Future and Industrial Design}

Underlying the philosophies of modernism, postmodernism and consumerism is a perception of products as static entities and of time as a problem to be denied, resisted or consumed. Emerging from this situation is the challenge for industrial designers to shift from the creators of products that are bound in a moment of time, to the creators of products that evolve through time to become meaningful artifacts. Consequently, industrial designers wil need to accept and embrace the idea of co-authorship. This will require an extension of the design process out from the factories and showrooms and into the hands and minds of their co-authors, the people making and using the products.

This process to look beyond the new product will require a vision into the future beyond today's 3D CAD models and into tomorrow's 4D visualising tools. Such tools would allow the compression of time. Designers and the co-authors of products will see today how the product will appear in the future.

that phenomenology, since it tries to understand our everyday relation to reality, offers a lot of insight into the relationship between people and products. In our view, this is what we should focus on in a materialistic approach. After the iconological deconstruction of products, we should not try to reconstruct them and create new icons. We should also try to understand what remains after this deconstruction: products as artefacts. 3 


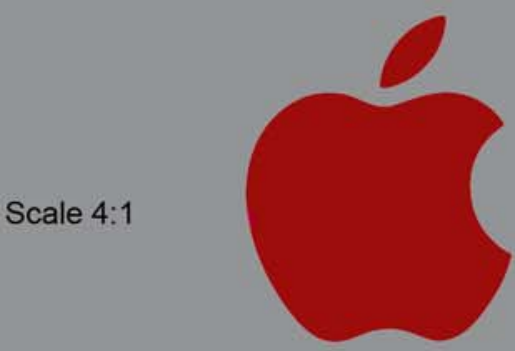

Designers

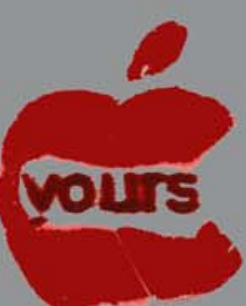

Co-authorship

\section{yours}

Owners

Brand to Possession

Sample \# 11.5

This experiment explores the transition from generic objects created by brands to specific artifacts created by individuals.

It was inspired by the problem of wearing of text on intensively used areas of hand held devices (eg buttons). This offered the potential to modify the communicated message relative to the intensity of its use.

The word "yours" was engraved into the surface (100 microns) and then painted over with a logo (10 microns) which filled the engraving with the paint colour. As the thin paint was proud of the surface, it was removed relatively quickly. The deeper, recessed text would only be removed as the material around it was eroded. By controlling the thickness of each layer, the speed of the transition can be controlled by the designer. 


\subsection{Designers as Co-authors}

The shift to co-authors will require designers to relinquish some of the control they traditionally have held over their products through defined production and marketing processes. However, it is perhaps this desire for complete control which has made their designs become, in a sense, sterile and unable to grow through time.

Products in a 4D design world would ideally be manufactured with the evidence of a clear creation history and a clear future that is revealed in front of the person who is considering having a relationship with it. The evidence that the designer intended the user to co-author the product offers a clear mandate that the user is supposed to leave evidence of themselves in the product. A pre-ordained path is revealed through time that the

designer has prepared by embedding design elements throughout the multiple, transformable skins of the product. This transformation is powered by

the touch of the hand, taking its life from the life of the person, interacting with it, forming a symbiotic relationship which augments the existence of both entities. 
Hand finished exterior All evidence of human involvement in the products creation are removed on the visible exterior

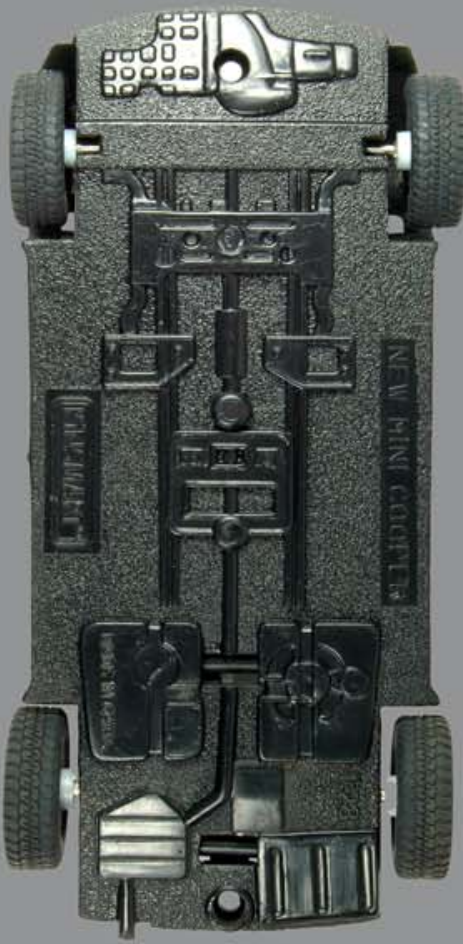

Unfinished interior The lines made by people during the products manufacture are not seen when the product is assembled, so are left unfinished.

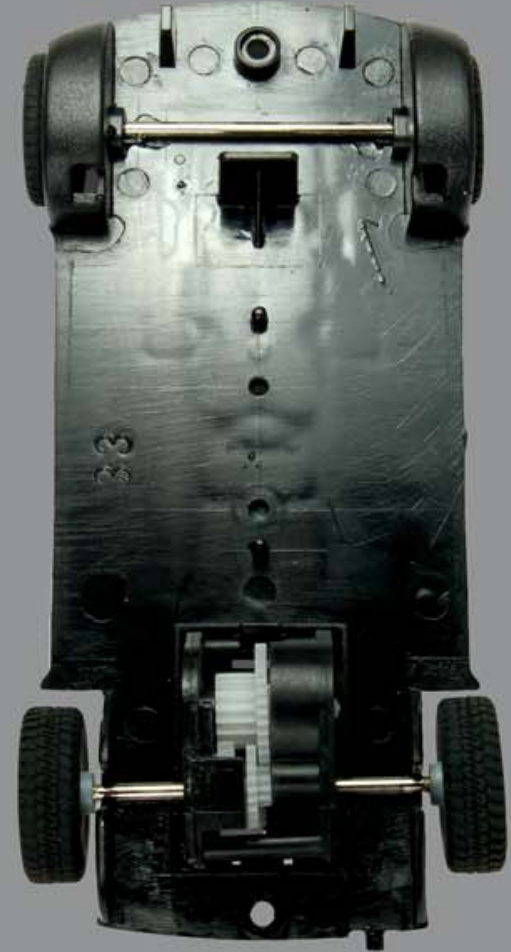

Plastic toy car (Injection moulded)

\section{Human Evidence}

The mass production of products is in fact still largely dependent on hand crafted processes, such as the hand polishing of injection moulding tools. The evidence of this human effort is intentionally removed from the visible surfaces of the finished product. This denial of human involvement leaves products impoverished of information about their creation history. The idea of a product being finished when it is sold reveals the limited role envisaged for the person using the object throughout its life. 


\subsection{Designed Evolution}

By finishing a product in the factory, the potential to improve it through use is denied. Without a designed pathway through life, a product moves away from the preordained vision of the designer into an uncertain and unplanned future. By exploring the idea of multiple layered finishes, where the removal of one layer reveals the existence of another, the idea of an product as ever being finished is questioned. This extends the concept of design durability or sustainabilty to another understanding of the life of a product as an ongoing and designed evolution. The term finishing the product now refers to the continuous process of fitting the product to the person.

Contemporary objects and spaces are cloaked in surfaces that have been enhanced, simulated, or engineered, surfaces that masquerade as other materials, surfaces where the physical and the virtual, the real and the imagined, collide. 
Gloss impression The gloss level of the phone impression suggests a harder material.

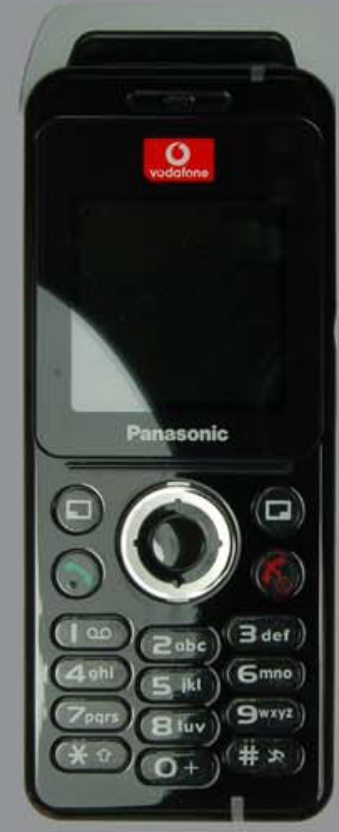

Panasonic Cell Phone Model X200

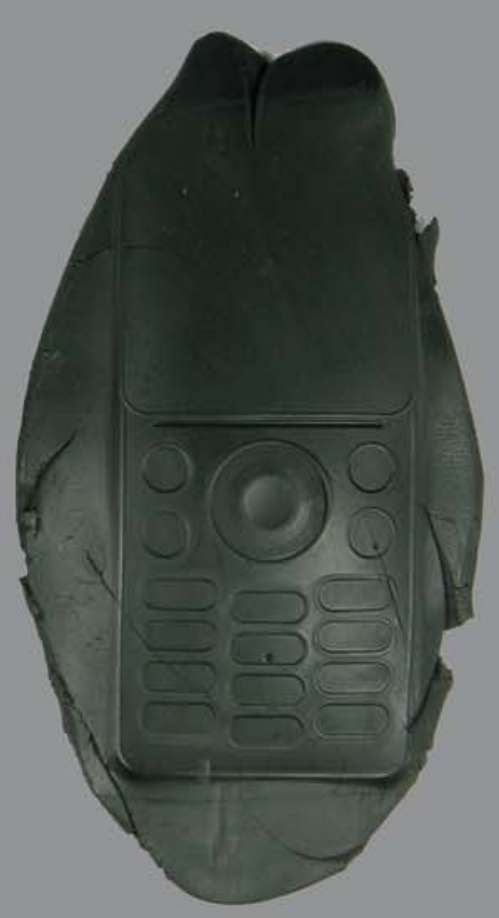

Actual performance The soft material is easily deformed, creating a discrepancy between suggested and actual performance.

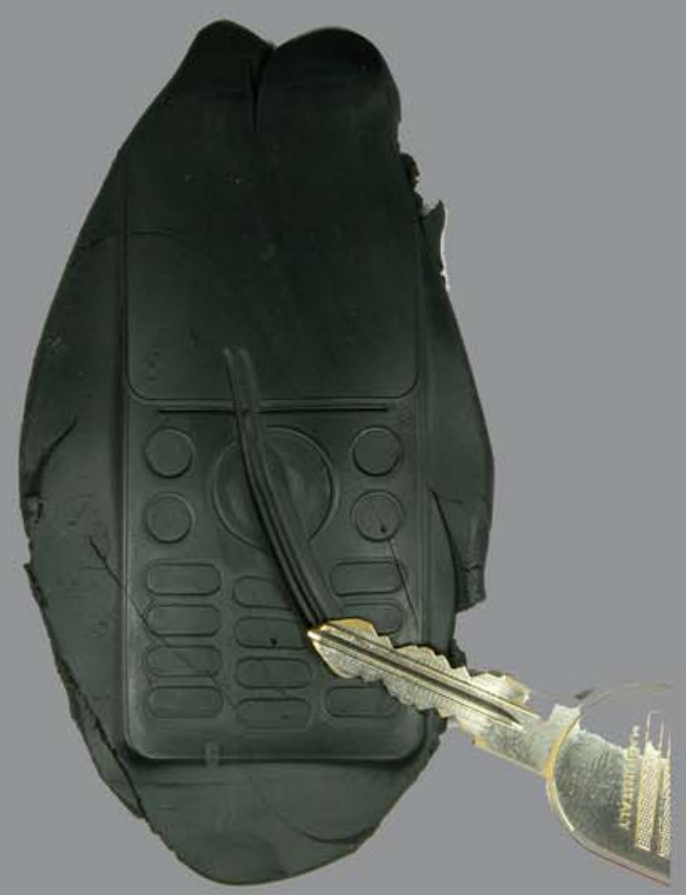

\section{Unsustainable Design}

The high gloss finish of this product limits the user's potential to touch it without damaging its immaculate surface. This problem is compounded by the contradiction of appearing to be a very hard material when it is in fact relatively soft and easily scratched. This deception has been created by highly polishing the mould into which the relatively soft plastic is cast, causing a discrepancy between the expected and actual performance of the material. 


\subsection{People as Creators}

The acknowledgment of the interaction between people and products as a form of customisation, personalisation and self-expression engages the potential for people to re-connect with the creation of their possessions. The combination of creating and using into one act allows the transformation from products into co-authored artifacts to occur as a process which is driven by the basic function of the product. The evidence of co-authorship will only be revealed through use and will constantly evolve until it is manifest as a discernible pattern of the user's effort, rituals, physiology, and shared life with the product.

It is the unmistakable tendency of modem economic and industrial progress to take out of the home all of the process of manufacture... One thing after another has been taken, until only cooking and cleaning are left and neither of these... leaves results behind to reward the worker as

did...spinning, weaving and soap making. What is cooked one hour is eaten the next: the cleaning of one day must be repeated the next, and the hopelessness of it all has sunk into women's souls

American Kitchen Magazine Vol. XII No. 6 (March 1900): 203. 

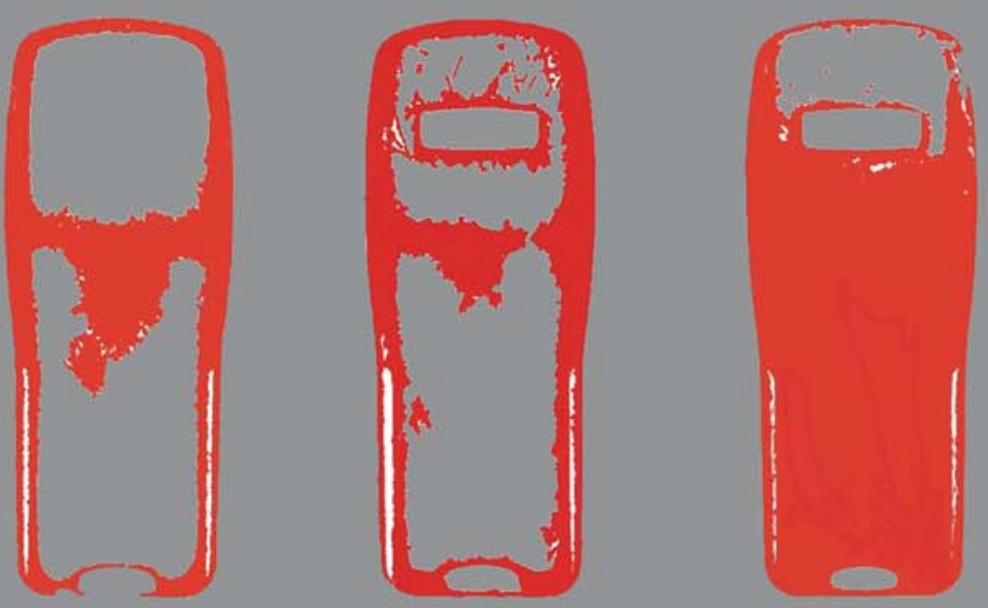

Back
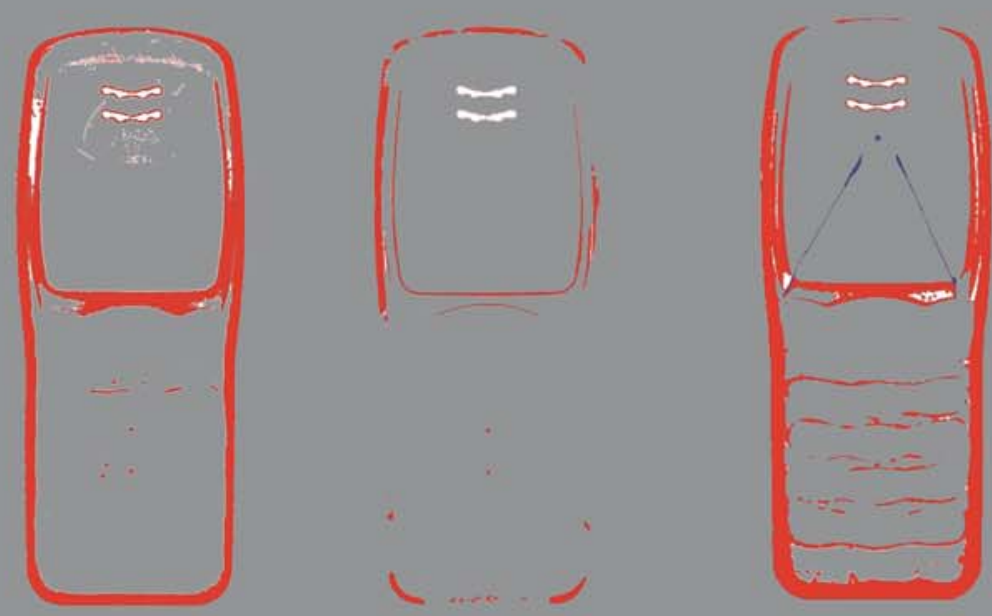

Wear maps

Individual Wear Patterns

This exploration was undertaken to quantify the diversity and individuality of wear patterns created on objects through use.

The surface wear in three identical cell phones (Nokia 3210) was mapped to allow a direct comparison of user created patterns. Each product has the same form and function, therefore the variations between the samples can only be attributed to the specific people using the products. 


\subsection{Personalising Products}

The meaning associated with a personal artifact is largely derived from its capacity to reveal information about the author of the wear (ref section 2.2). Without a direct memory or proof of the author, the ability to attribute meaning to the artifact is questioned. By modifying the product with a distinct personal mark, the identity of the owner can be permanently linked to the product.

Wax seals have traditionally been used to add personal details to products (letters). The use of a pre-made stamp (signet) allowed for precise and difficult to duplicate impressions to be easily made into warm wax. A variation on this process was trailed to explore the potential of contemporary ultraviolet cured resin to create a simple yet durable unique mark.

An area on the back of the product is used to imprint information specific to the store that sold the product and to the person who bought it. This information allows the product to become unique during the purchase process establishing a permanent record of the product's origins and owner Imprinting creates a purchasing experience that acknowledges the product's physical and emotional change of ownership and the beginning of the product's meaningful life.

A light proof foil is used to inhibit the curing of the ultraviolet sensitive paste into which the imprints are made. When the product is sold the foil is removed and information specific to the store and new owner are manually imprinted. The product is then placed under an ultraviolet light that cures the paste. Recessing the imprint below the product's surface and the hard nature of the paste derived from dental technology establishes a imprint that will endure the lifetime of the product. 


\section{Store Logo and Purchase Date}

The relationship between the store selling the product and the new owner is made evident by the permanent impression of the store's logo and the date of purchase. This information is important for warranty claims and the continued support needed to operate increasingly complex devices.

Technological improvements to hand held electronic products mean that they are often replaced by newer more capable versions, creating a cycle of repeat sales. The permanent store logo creates direct evidence of the relationship between the product and the store that sold and supported it throughout its life, increasing the likelihood of an ongoing relationship.

\section{Body Extension}

An impression of the new owner's fingerprint is made into the product signifying that it has become an extension of their physiology. This is the first evidence of a relationship between the user and the product. Over time additional evidence is added, still authored by the finger, but softer and blurred by time. The speed at which the product changes from the instant remoulding of the finger impression to the gradual erosion of the product multiple surfaces reveals the diverse aesthetic qualities achievable through reactive $4 \mathrm{D}$ techniques. 


\subsection{Software Design}

If the user has a role in directing the patterns that form in a product's surface, so does the software designer that decides on the layout and sequence of controls. The pattern of use becomes exposed in the interface through the controls that are most frequently "picked on", such as ON/OFF which is used every time the product is operated. The type of touch, a downward press or a sideways slide, each manifests into discernible patterns. These patterns are a synthesis of user's habits and of designer's edicts with neither fully aware of their role in creating a choreography of use.

The connective wiring of the body extends beyond it ostensible limits to the cultural networks within which it is inscribed. With lines of communication that turn everything inside-out and outside-in, bodies are prostheses of machines as much as machines are prostheses of bodies. The interfacing of the so-called natural and artificial creates forms that are always mutating. 6 

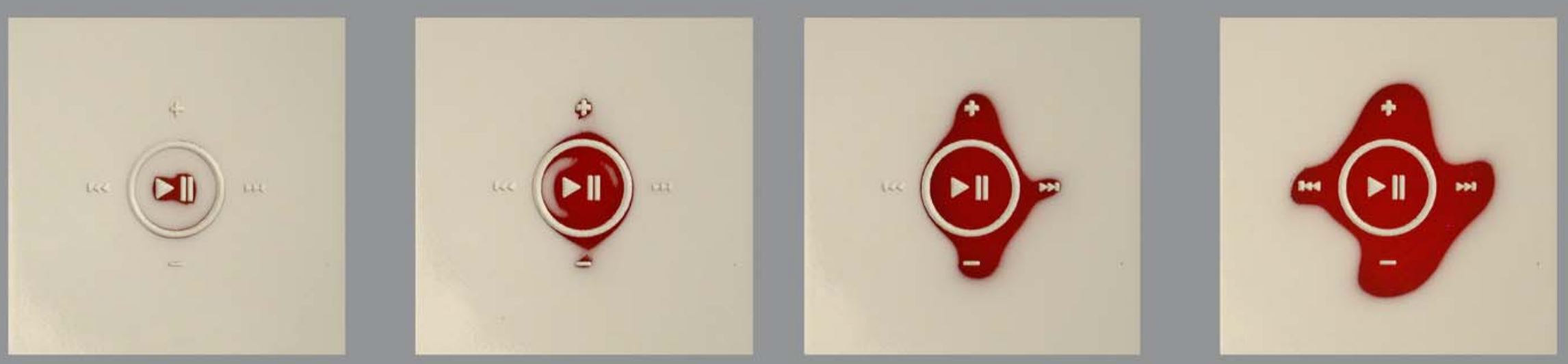

Interface Patterns Sample \# 10.8

A product's interface forms a "site of touch" which defines the elements of location, frequency, direction and force. 


\subsection{Physical Limitations}

As products become more capable of complex processes, the fingers become the restriction between human thought and physical action Contemporary products can serve multiple functions and require new forms of dexterity increasing beyond the finger's capabilities. Typing on a laptop keyboard is difficult enough compared to a standard keyboard but the growing need to send text messages on small portable devices elevates this restriction to a more concerning level.

Is the very wear being discussed and promoted as a valuable design asset, in fact simply a result of an inefficient interface?

It is arguable that the amount of wear shown in a product is a measure of the efficiency of the interface. If we create a poor interface that is not considered from a 4D perspective, it focuses the wear on specific parts that become worn and eventually stop functioning. If this happens to a product, it is simply replaced. For the hand, however, if it is worn past its ability to regenerate, it cannot simply be replaced.

Each year, 3.8 million people complain of injuries resulting from text messaging, according to the nationwide survey by Virgin Mobile. Each day almost $100 \mathrm{~m}$ texts are sent in the UK - so perhaps it is no surprise that 38 percent of us suffer from sore wrists and thumbs as a result. 7 


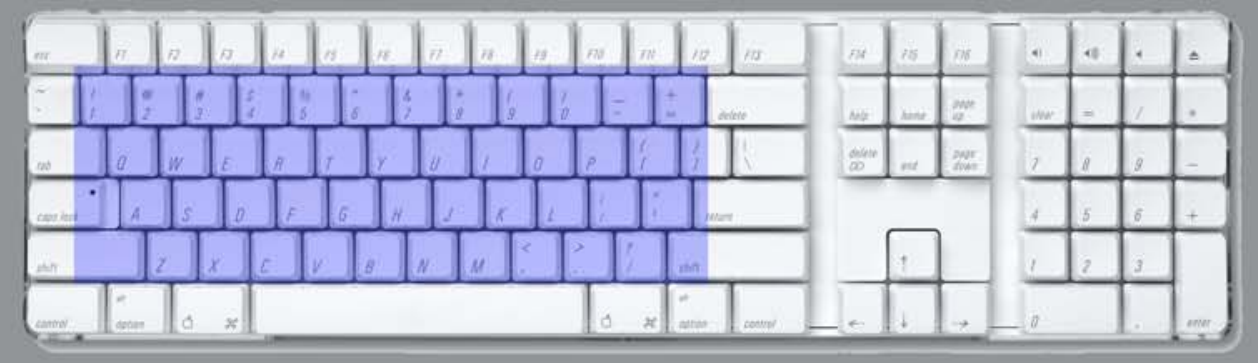

Apple Wireless Keyboard

\section{Smaller Keyboards}

The dimensions of the interface influences wear intensity.

\section{Wear Revealing Damage}

Wear patterns could be used as a warning of overuse to encourage a change in behavior. 


\subsection{Customising Through Use}

When using electronic devices such as typing on a keyboard, the repetition and dexterity required creates a complex and increasingly intuitive action. Over time this action leaves evidence in the form of erosion in the keys, relative to the frequency of their use. This erosion forms a unique pattern which has the potential to transform a generic keyboard into a customised version with individual landmarks that collectively form a cognitive map.

The combinations of tactile and visual information developed through use locates the user within the specific site that has been customised through their interaction with the product.

Coatings that were designed to be removed through use, revealing where and how often the products was touched could allow this customisation to occur within a simple to manufacture yet context specific solution.

The fact that computers are primarily designed to accommodate a broad range of functionality and thus have a standardized interaction-style has two implications. First, it means that computer offer very limited opportunities to design for interaction; all physical aspect of interaction are left out of the design process. Second, it means that computers offer limited opportunities for the information-for-use to be meaningful for function. Therefore usability aspects of computers are not about the interaction-style but about the procedures for operating the computer. 

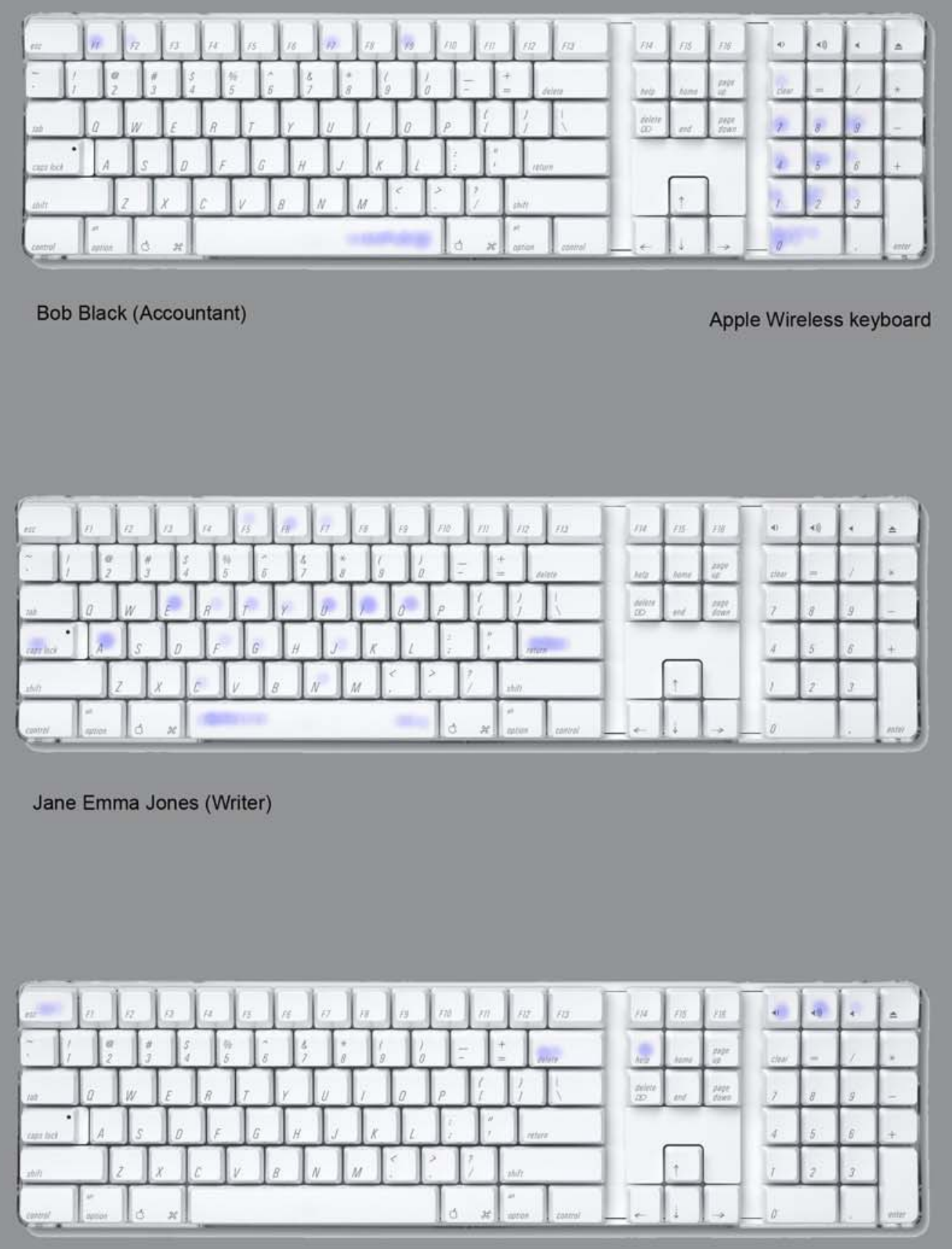

Michael P Silcock (Musician) 


\subsection{Associations in Products}

The precision used to define the pattern in the surface of the teacups on the opposite page suggests a deliberate, considered and designed act. The typically negative understanding of staining as a chaotic and detrimental occurrence has been reinterpreted as a positive act that highlights the graphic pattern through use. The underlying association with stains and hygiene remains embedded in the meaning of the product and this creates conflicting associations within the products. While the stains are deliberate they are also related to dirt and this association could influence the experience of drinking tea

The 'iPod' mentioned by the designer of the tea cups (opposite page) as an example that conforms to 'the assumption that use is damaging to a product" also has a historic association between wear in the external surface of the product and an assumption of wear in the non-visible internal electronic mechanism. To allow wear to be seen as a constructive rather than destructive act requires an understanding and a response to this association. The emergence of electronic devices with no internal moving parts and thus no wear mechanism, offers a response allowing wear on the product's external surface to be seen without negative durability implications. 

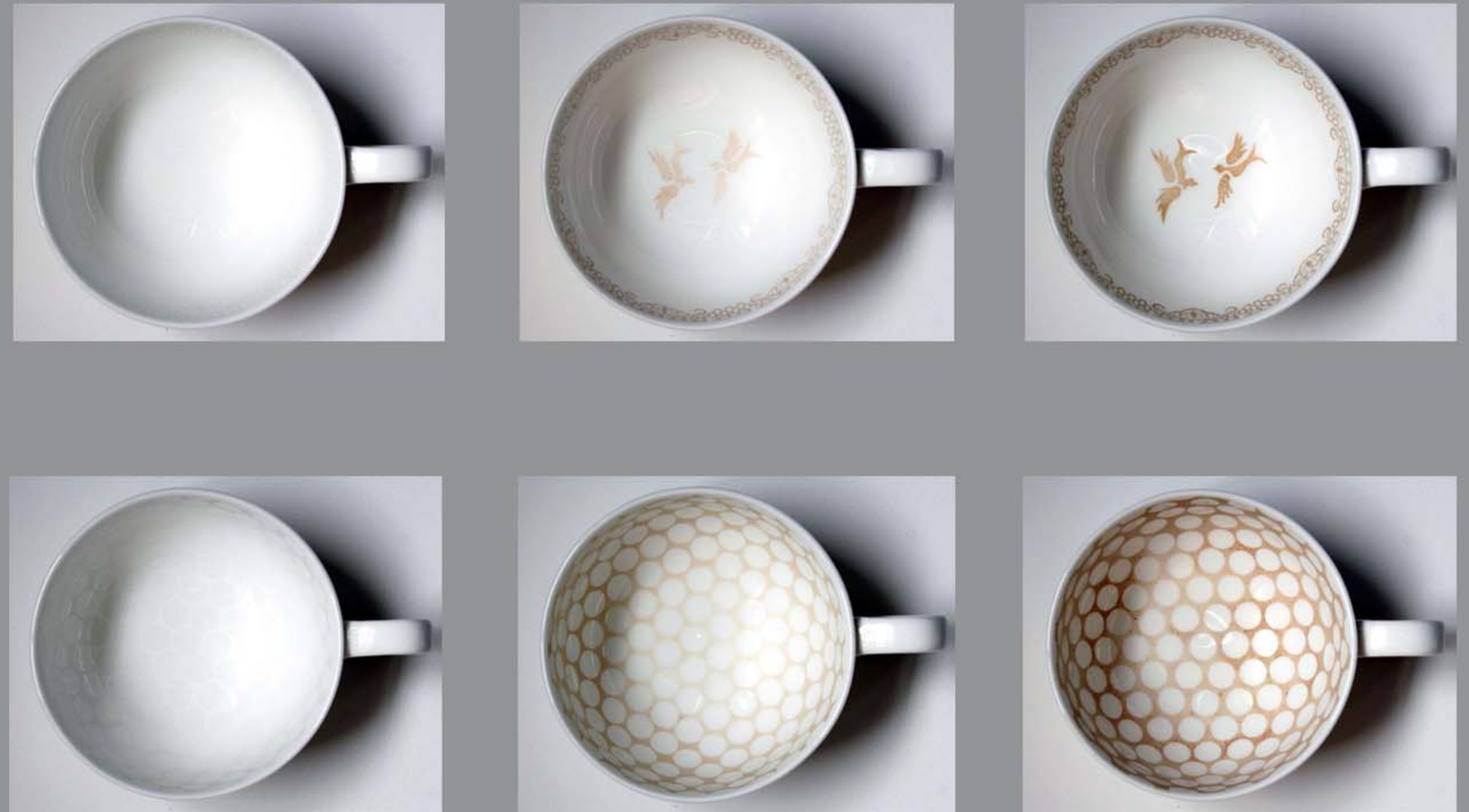

Designer: Bethan Laura Wood

Stain is a set of a teacups designed to improve through use. This project examines the assumption that use is damaging to a product (For example, scratches on an iPod). 


\subsection{Autonomous Skins}

While the products shown on the opposite page appear to be externally and internally similar below the surface, there are fundamental differences that have important implications about our understanding of wear and its meaning.

The iPod uses a disk that spins at 16,000RPM to store information. However the Nano and Shuffle have no internal moving parts, eliminating the wear created through friction between moving components. This challenges the fundamental associations between external and internal wear. The product skins become autonomous of their internal frailties and become free to respond to the user's touch without mechanical implications.

The 'Apple Shuffle' from this range of products has been selected as the generic product upon which the applied design experiments have been based. It was selected because it offered a simple form, suitable for multiple graphic patterns, was small and intensely used, is durable due to solid state electronics and is readily available at a reasonable price.

It's in the sexy but vaguely absurd mode of Raymond Loewy's streamlined pencil-sharpener, a period artefact that was mocked by Henry Dreyfuss. Loewy had slipped a sleek, handsome monocoque shell over the pencil sharpener, but inside, both he and Dreyfuss knew that it still had the same old grinding mechanical guts. 10 
Apple Portable Music Players Image source: http:l/lmww.apple.com/nzititunes/

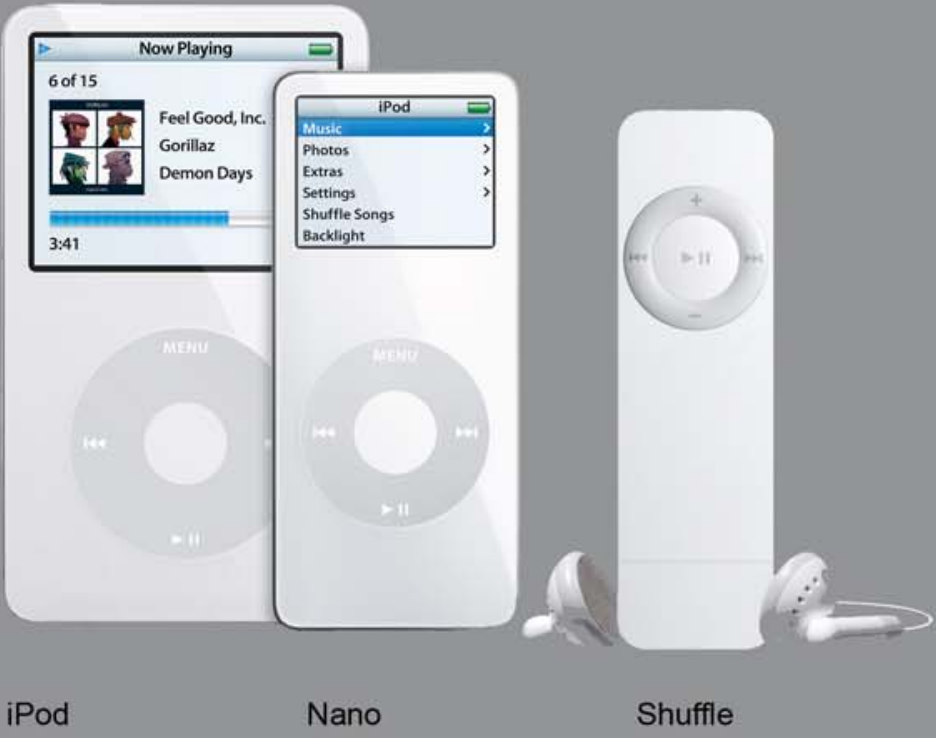

Internal Differences

The external similarities between these products conceals fundamental internal differences. 

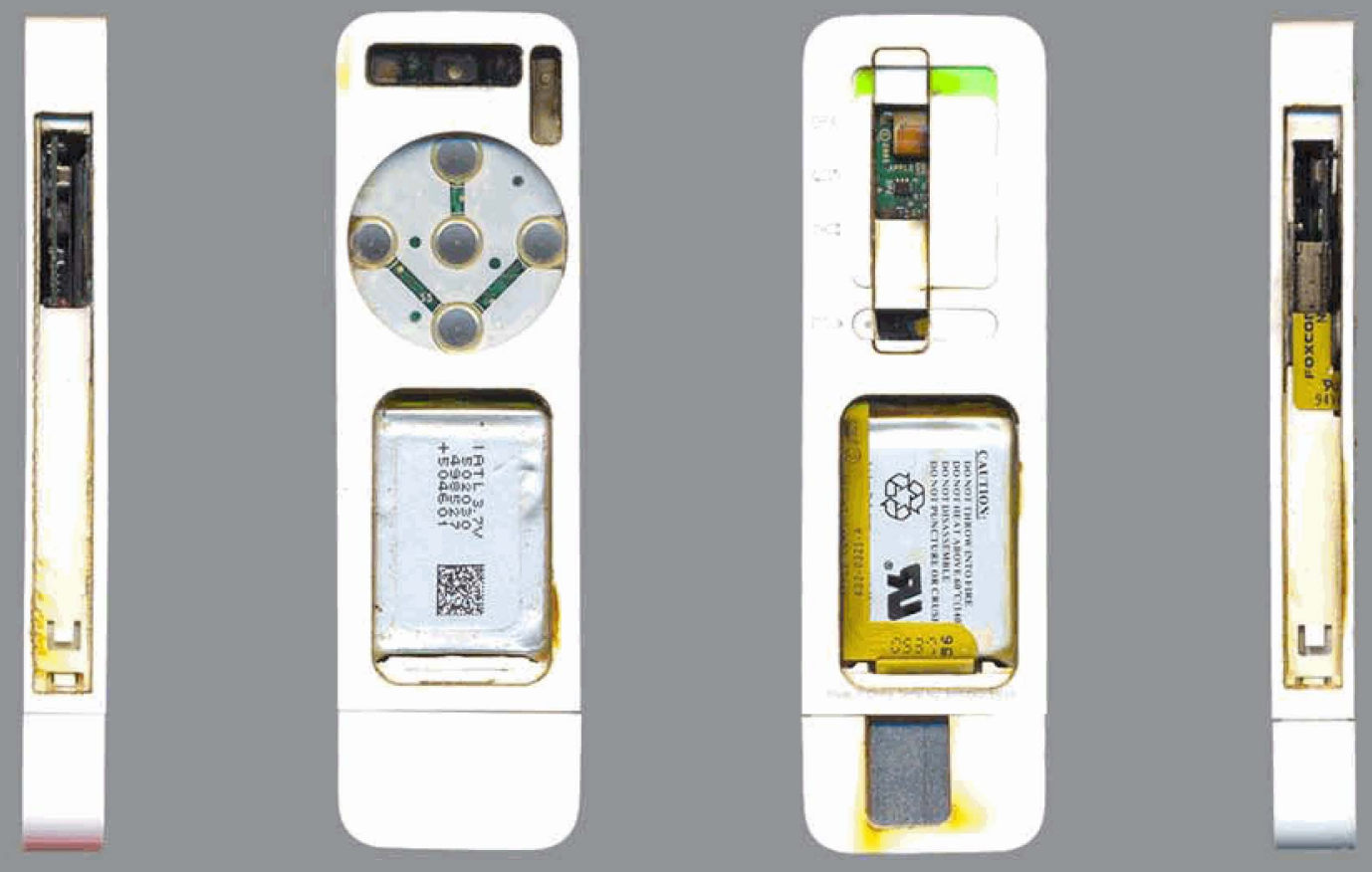

(Laser dissected) Apple Shuffle

No Moving Parts

An investigation was undertaken to compare the types of internal mechanism used in the Apple Suffle, Nano and iPod 


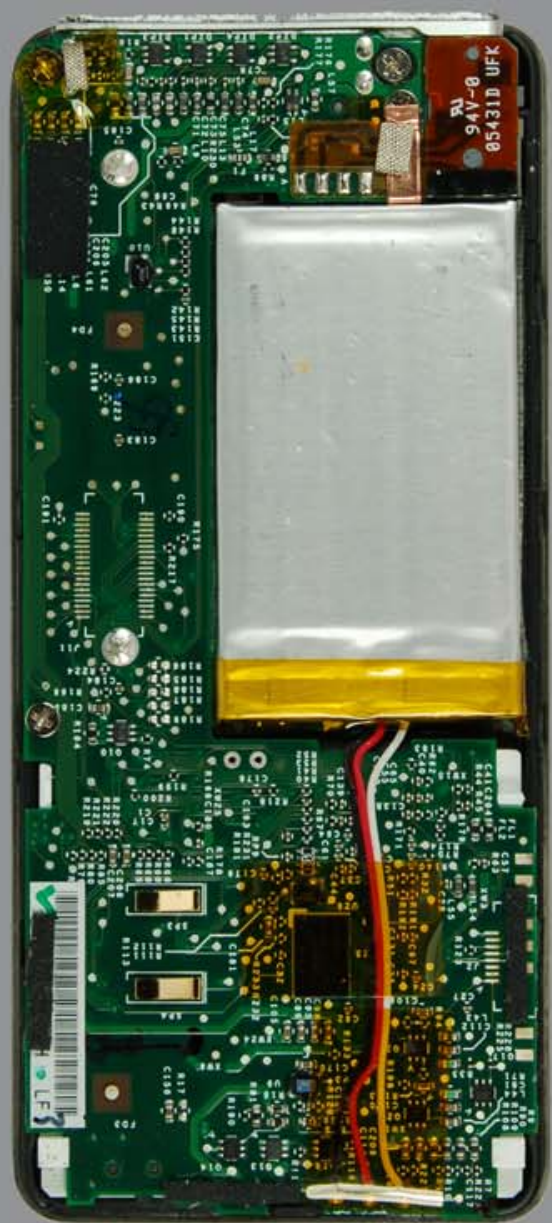

Nano

The internal mechanism of an Apple Nano is based on FLASH memory that contains no moving parts.

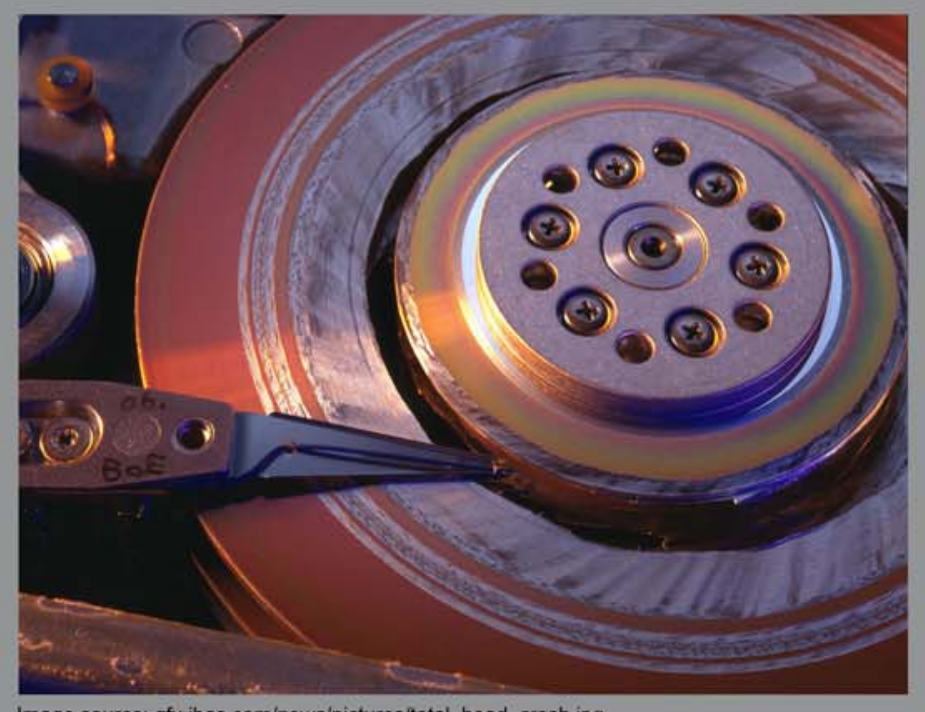

Image source: gfx.ibas.com/news/pictures/total_head_crash.jpg

iPod

The iPod uses a hard drive to store data. This image is of a crashed hard drive from a computer (iPod was unavailable). It reveals the dynamic nature of the mechanism and thus susceptibility to wear. 


\subsection{Generic Commodities}

The continued reduction in the size and cost of electronic products coupled with an increase in performance have been key factors influencing the constant replacement of products. Limits are now being reached in all three of these areas. Finger size is now dictating product size, products already perform beyond our needs, and they remain relatively cheap. This means that products are becoming generic commodities that have little meaning or value to people. As these products technologically mature and the reasons to replace them diminish, the potential for more enduring relationships emerges. 


$$
t
$$




\subsection{Conclusion}

"jams whorepod" evidences and substantiates the potential for contemporary portable electronic devices to become meaningful personal artifacts. The original designer's vision of this device (Apple shuffle) as a generic, functional and minimal product has been challenged by the product's owner/co-designer to become a personal, humorous and diverse artifact.

The slang name "jams" engraved into the surface of the product denotes the product as belonging to someone (Jam). The term "whorepod" reveals an attempt at humour and a casual and confident understanding of technology. The alligator graphic reveals an interest in images and symbols. The extensive wear on the edges reveals the users intense use and thus interest in music.

This information, if perceived and translated, reveals a diverse range of personal information about the owner. The content evidenced in the products surface creates a meaningful artifact that may eventuate as an heirloom from an inexpensive product with a relatively short life span. This potential to become an intergenerational heirloom could be enhanced through increasingly durable solid state electronic technology or through interchangeable technology that allows for constant upgrading of the internal technology while preserving the product's external skin and thus, personal legacy.

This product's long term meaning and value were not generated through the maintenance of the designer's vision but from a deliberate deviation from it. This raises the critical question of the role industrial designers have in isolating suitable products that have potential to become meaningful artifacts and what qualities the products need to become effective vehicles for physical user generated content. 

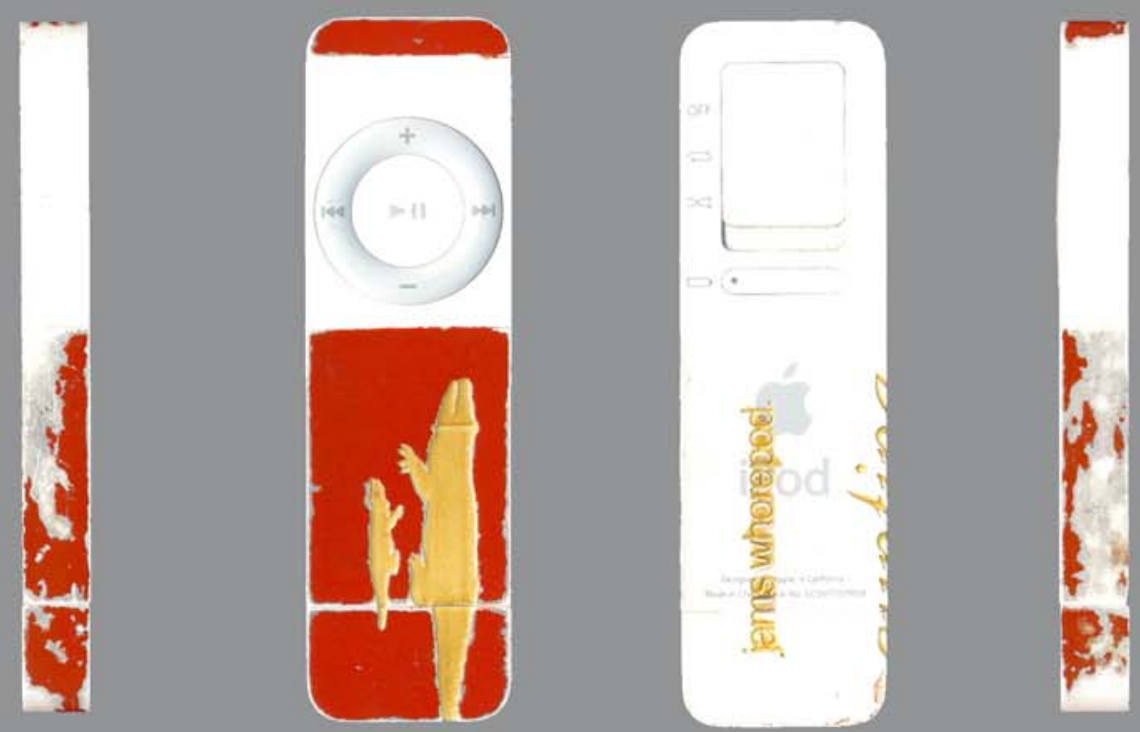

Apple Shuffle or jams whorepod? 

3. Manufacturing Artifacts 
This chapter explores the capacity to manufacture distinct artifacts that reveal discernable creation history.

The deterioration comes not because of bad workmanship in mass production but because the range of qualities which mass production is capable of just now is so dismally restricted; because each is so uniform and because nearly all lack depth, subtlety, overtones, variegation, diversity, or what ever you choose to call that which distinguishes the workmanship of a Stradivarius violin, or something much rougher like a modern ring-net boat. The

workmanship of a motor-car is something to marvel at, but a street full of parked cars is jejune and depressing; as if the same short tune of clear unmodulated notes were being endlessly repeated. A harbour full of fishing boat is another matter. Why do we accept this is inevitable? We made it so and we can unmake it. Unless workmanship comes to be understood and appreciated for the art it is, our environment will loose much of the qualities it still retains.11

\subsection{Visual Diversity}

David Pye's argument that the range of qualities achievable through mass production is "dismally restricted" is based not on the potential of the processes but how the processes have been utilised by designers. Variation inevitably exists in all production processes yet it has been reduced through refined processes and tolerances that conceal its potential diversity. If harnessed, it can be utilised to manufacture products that are visually unique, expressive of the process used in their creation and yet, consistent in the way they perform. The image on the opposite page of three visually distinct surface patterns on common products (timber screws) illustrates the potential to create mass produced yet distinct artifacts. 


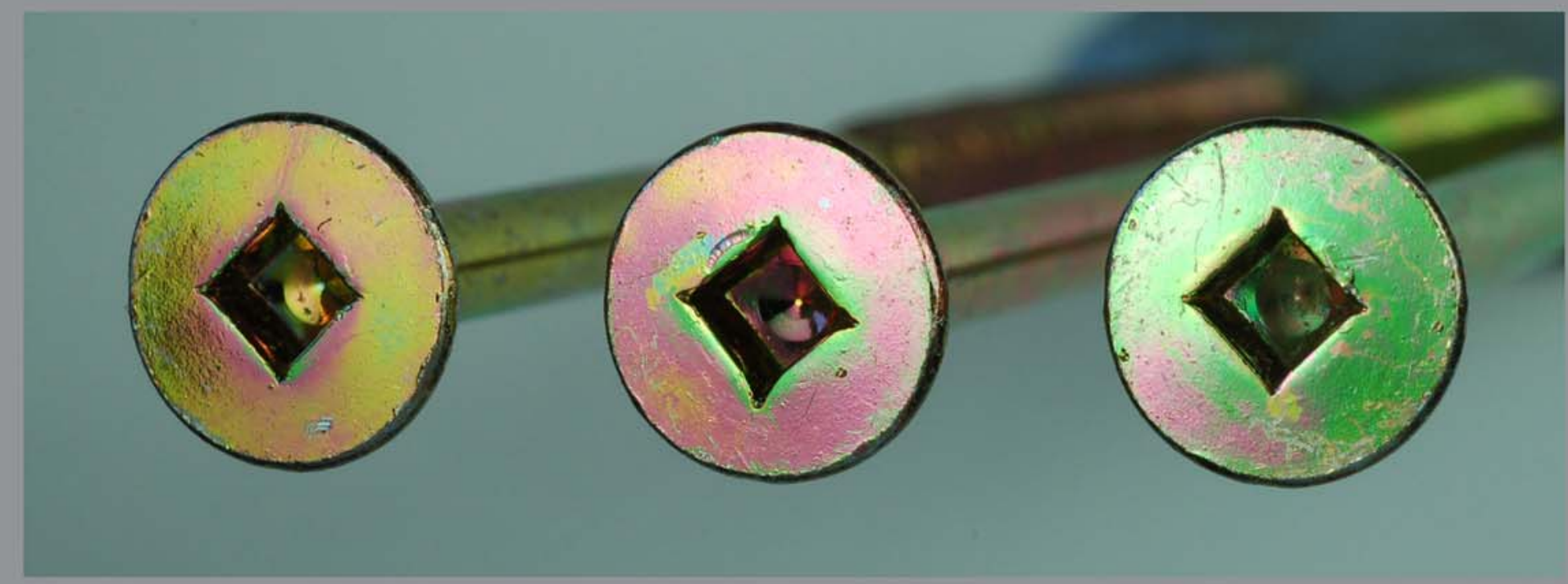

Csk Squaredrive Jet Screws (passivated zinc plating

Mass Produced Diversity

The individual screws are visibly distinct yet they are produced using a standard and cost effective process (The visual diversity does not affect the functional interchangeability of the product) 

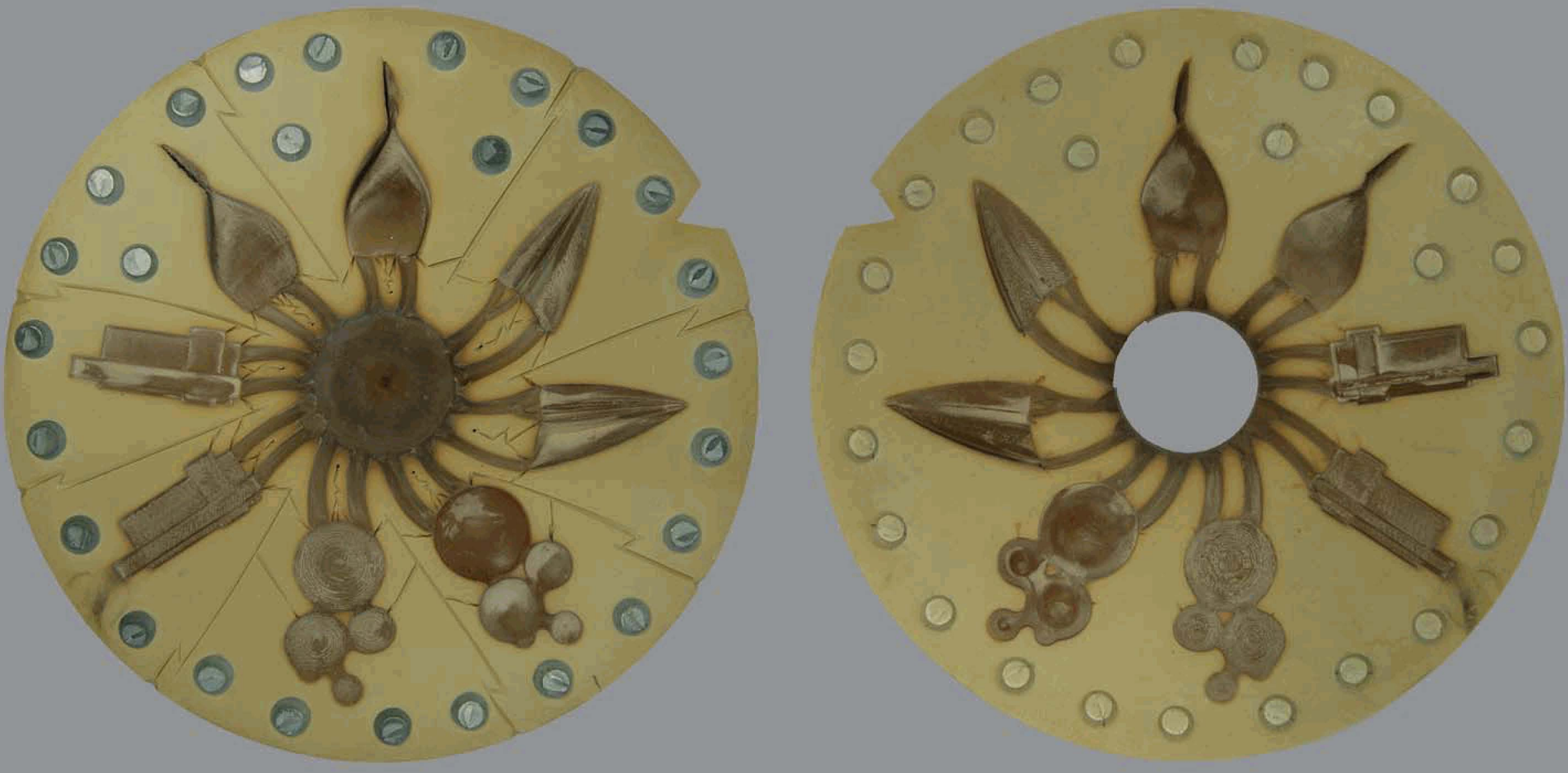

Centrifugal casting moulds (High temperature vulcanized rubber)

\subsection{Conforming Technology}

The function of a mould is to constrain a material in all directions. By definition this means there is limited potential to create products each with an individually distinct form or surface texture using moulds.

These moulds were used in the design experiments to to translate the individual plastic 3D prints into multiple zinc castings. Mutliples of the product were required to conserve a 3D sample of the specific stages throughout the products life (Ref samples \# 27-136). 


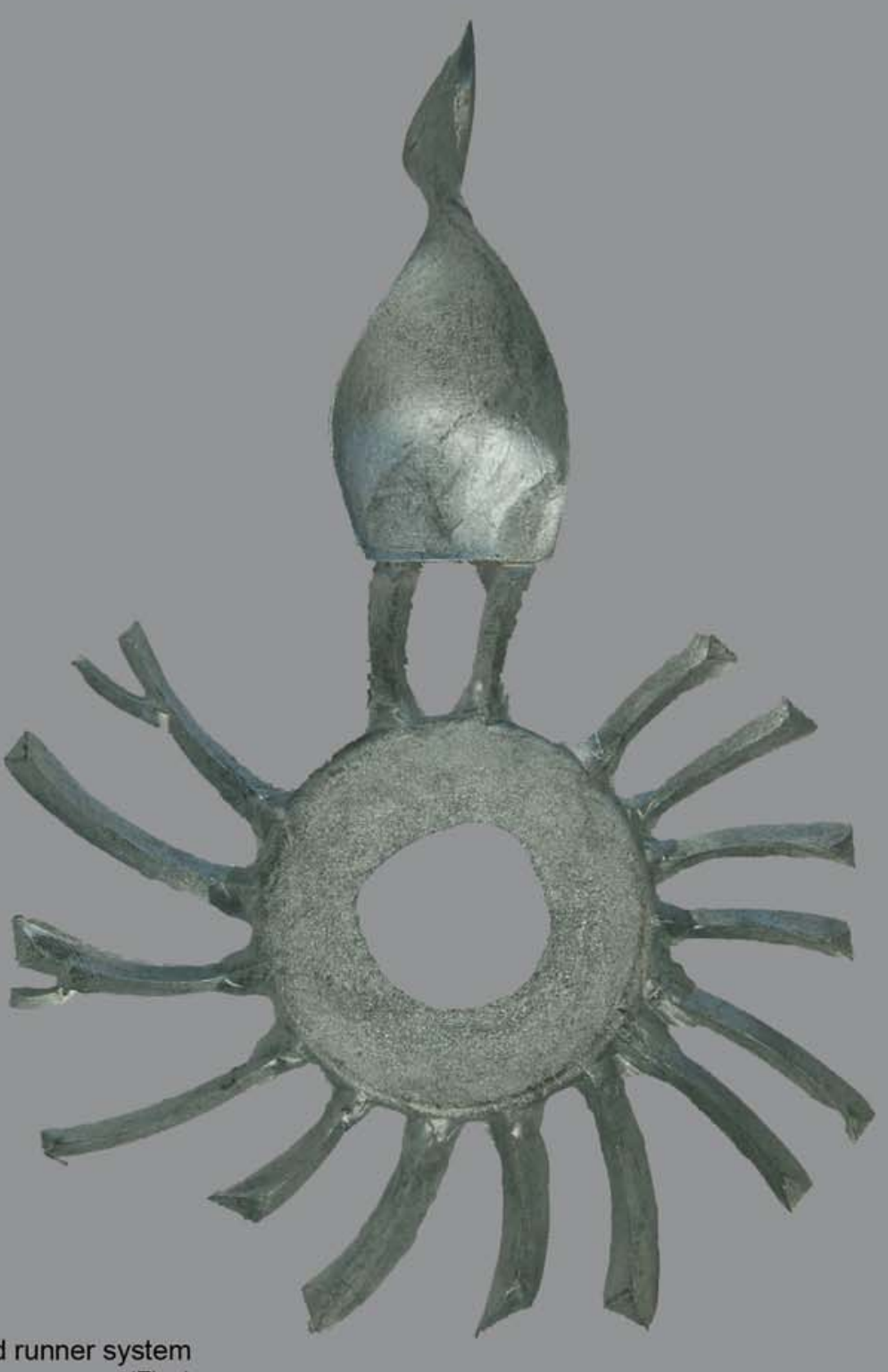

Unfinished casting and runner system
(Zinc)

\subsection{Manufacturing Multiples}

Through the use of moulds, large numbers of products can be produced that are uniform in shape.

While the shape of moulded products is dictated, potential variability emerges from the path the material takes throug

the mould (ref image) and the coating

used to finish the product. 

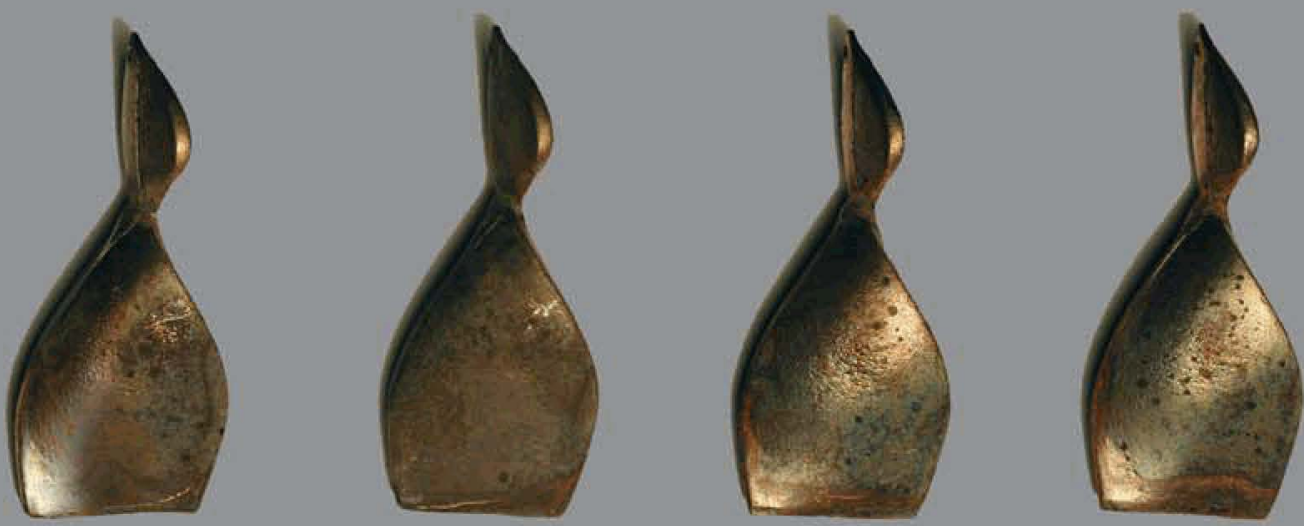

\subsection{Reactive Metals}

Variability inherent in the oxidation process transformed the initially identical products into visibly distinct products.

The reactive quality of brass and copper continues to oxidise throughout the

product's life. Atmospheric conditions form

the oxidations and use removes it, creating

a dynamic and symbiotic relationship.

The electroplate copper samples were oxidised through exposure to the atmosphere for three months.
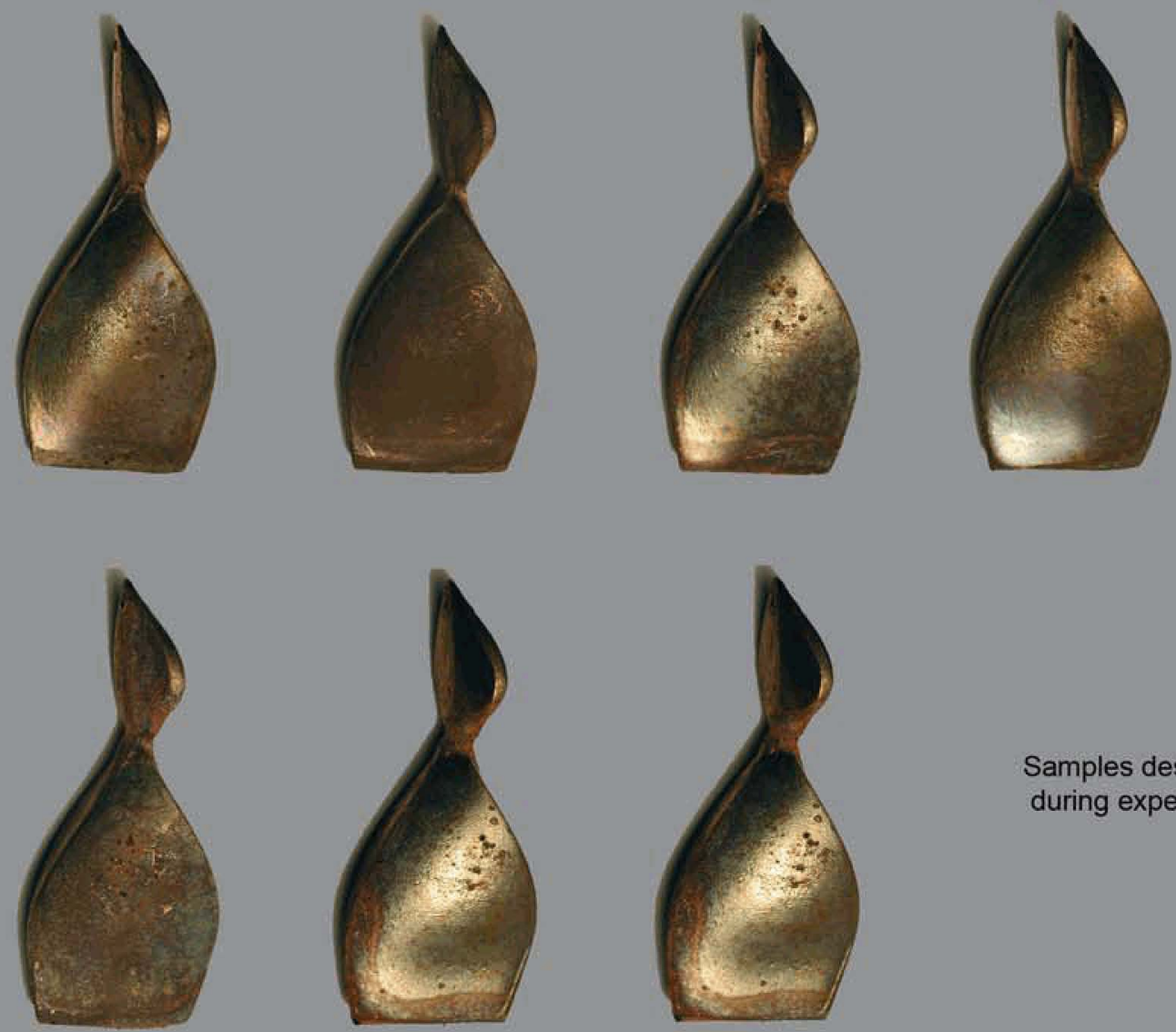

Samples destroyed

during experiment. 

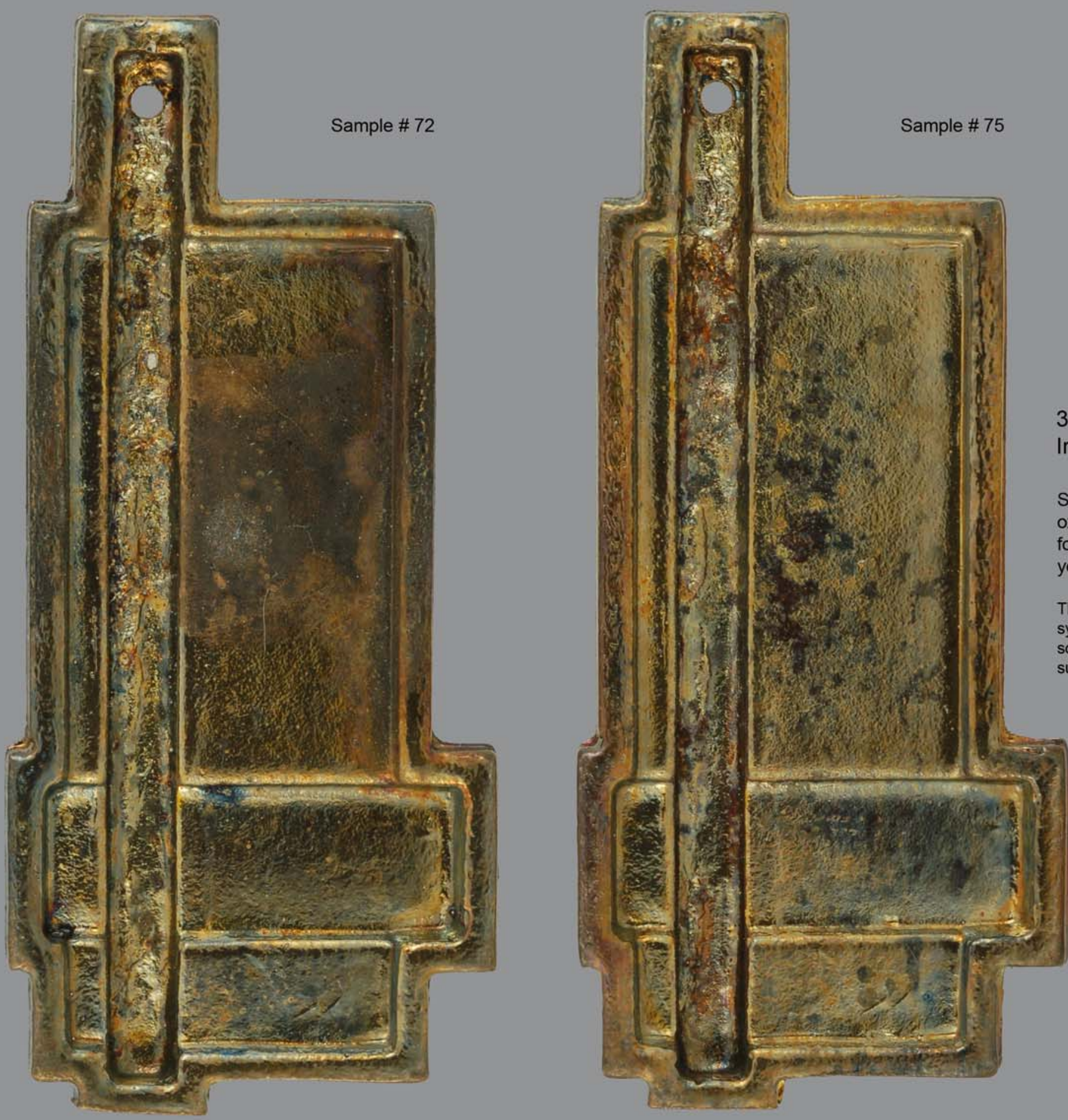

3.5 Process and Form Interactions

Submersion during the plating and oxidation process, combined with the form of the product, created a varied yet shape specific surface finish.

The electroplated brass samples were synthetically oxidised by submersion in a solution of deionized water and liver of sulphur for three minutes. 


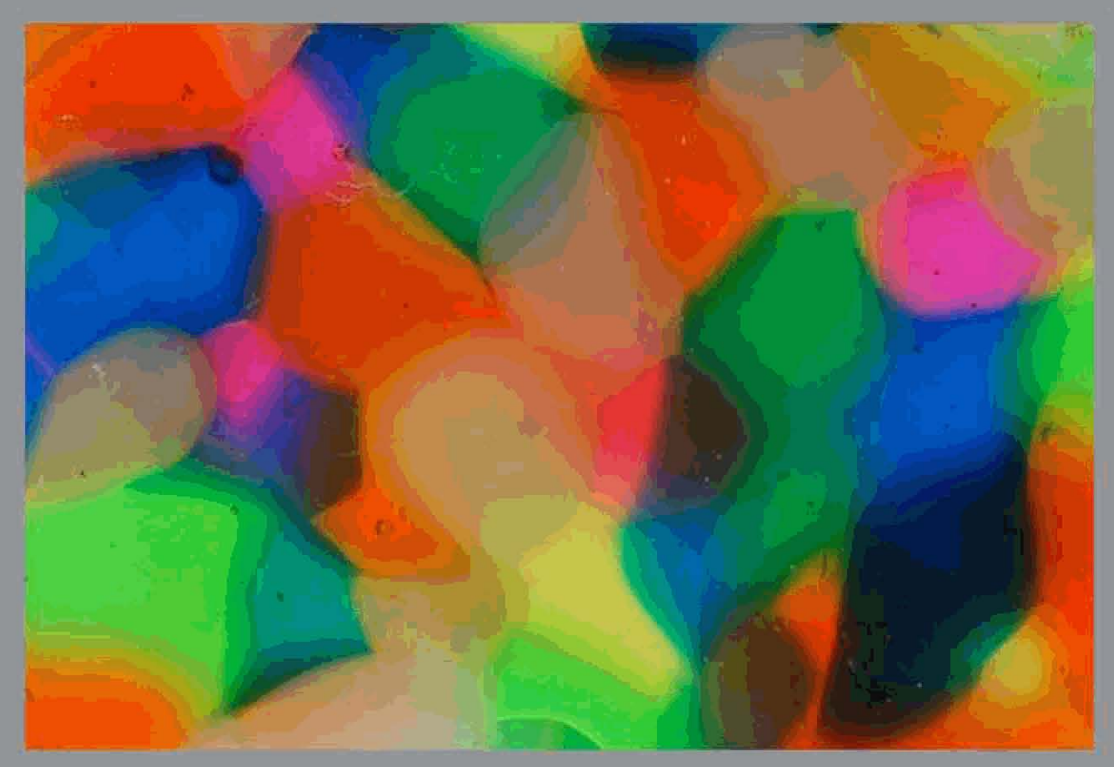

Heat pressed thermoform plastic sheet

\subsection{Unique Material}

The fusion between the coloured plastic granules visable in the material reveal the viscosity and technique used in its creation. The manual processes of filling the mould with coloured granules is inherently variable and this allows the creation of a visually distinct yet functionally consistent material. 


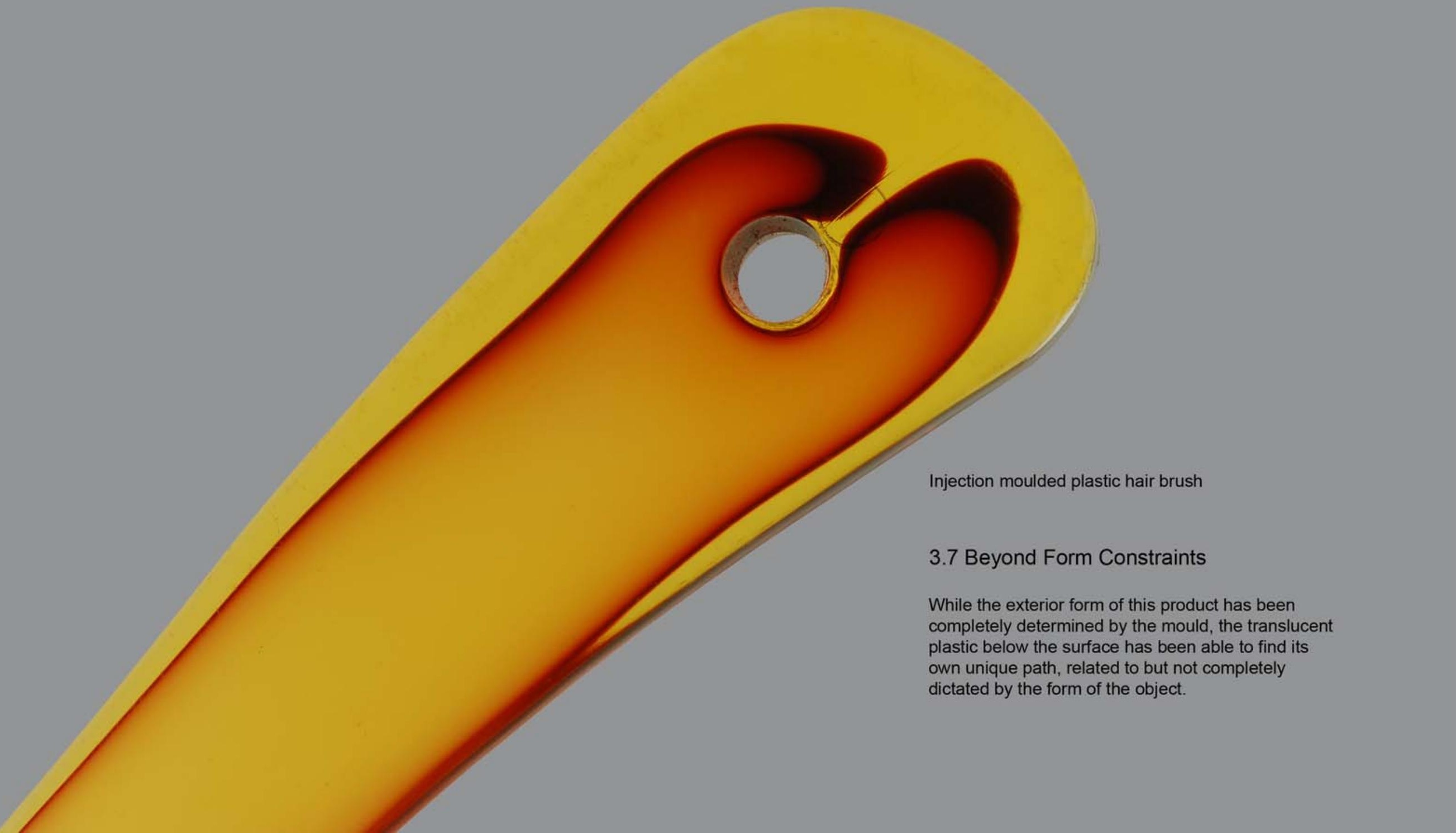


The most typical and familiar example of the workmanship of risk is writing with a pen, and the workmanship of certainty, moderm printing.12

\subsection{Creation History}

David Pye has suggested the way products are perceived and valued is directly linked to the risk involved in their creation termed the "workmanship of risk". This proposes that the marks left by a craftsperson give witness to their mastery of a given process. For example, each chisel mark of a master carver can be seen and assessed for the physical skill left evident in the surface texture of the carved timber. If the surface is sanded flat, it removes these marks. Hence a less skilled person can remove the evidence of their poor craft by simply using a lot of risk free work.

In consideration of this theory, the removal of all evidence of a product's creation makes it difficult to decipher the tectonic process used in its construction. The product is thus left in an attempt to preserve its moment of instant creation. (A cell phone cover takes approximately 20 seconds to go from raw material into a completely finished part, accurate in form, colour and surface texture). No product or material is capable of completely resisting the actions of time and use. When the product inevitably yields it is not part of an already established tectonic process created during the making of the product. Without an established and evident history, the product has no relationship with its past and thereby its future.

With limited potential for electronic products to be mass produced by hand, the challenge is to create products through machines that are more expressive and demonstrative of their creation history. 


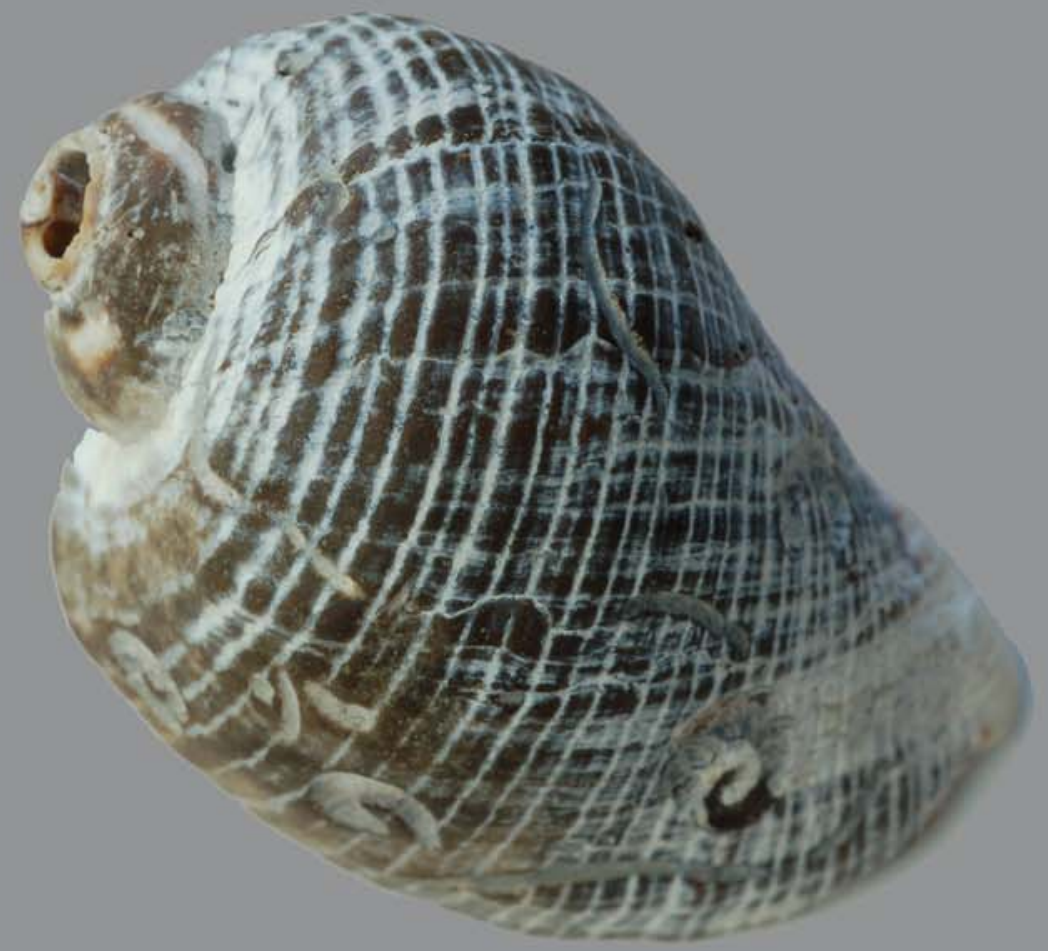

Visible creation history

This object (shell) reveals clear evidence of its creation history in the lines that witness its layered construction.

Additional information (worm marks) have also accumulated during its life. 


\subsection{Rapid Prototyping}

The search for processes that are both distinct and demonstrative of creation history inevitably led to rapid prototyping technologies. They are emerging as a new category of machines with distinct aesthetic qualities that are directly related to and expressive of time.

This technology is seemingly chaotic and contrary to the concept of machines as simple ordered entities. Rapid prototyping creates products by depositing material in sequential layers similar to cellular growth in organic matter. As a new technology, it is surprisingly familiar and related to the natural world unlike the machines of the industrial revolution that were distinctly separate. Each layer builds on the previous one creating a surface and mass with a discernible construction history. One of the processes which was selected for its visible construction lines is fuse deposition

modelling (FDM). Fine continous beads of plastic ( $0.3 \mathrm{~mm} \mathrm{ABS}$ ) are used, so each erratic construction path followed is left evident in the final product

Efforts are being made to remove the evidence of the layers and construction paths to allow the creation of products which are indicative of current smooth and flawless mouldings. Yet it is the flaws that humanize and connect these objects to time

Within the rapid prototyping industry from which these machines are emerging, the process of manufacturing a component has returned to the familiar term of "growing". This suggests a more cyclic understanding of product creation. 


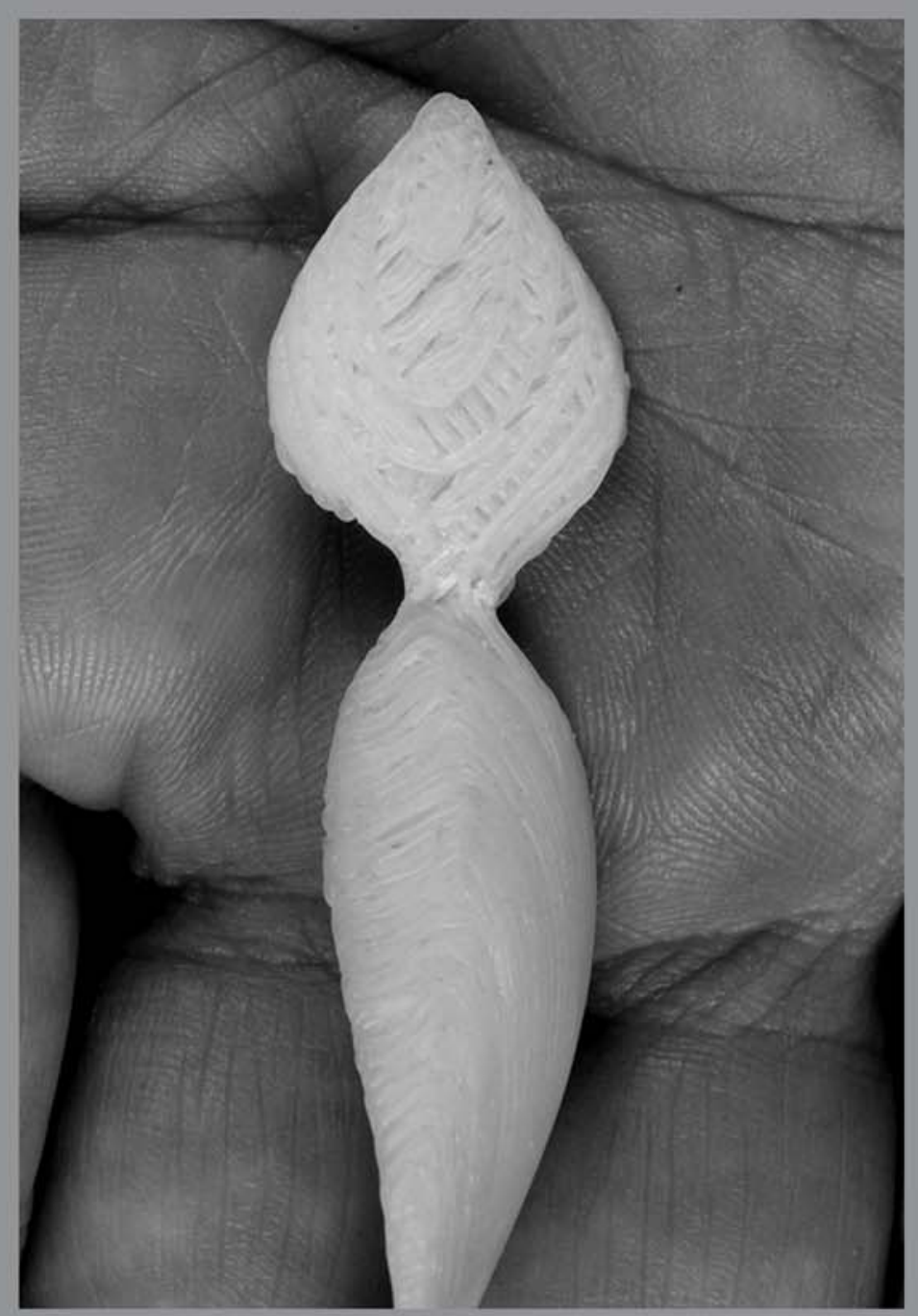

It violated the neat boundary between what is inside the computer and what is outside. In a strange way, holding the part felt almost like touching the soul of the machine.13

\section{Rapid Prototyping Technology}

Fuse deposition model (FDM)

Sample \# 8.5 


\subsection{Digital Uncertainty}

Drawing a vertical or horizontal line on a computer seems simple for the program to calculate and define but as the line is moved slightly off either vertical or horizontal axis, the certainty of a binary measurement becomes questioned. It is no longer 0 or 1 but 0000.1 .

As these measurements diminish, they extend beyond the tolerances of the hardware and software. This creates subtle variables and inconsistencies in the outcomes which have the potential to create distinct products; one of the qualities explored in this reseach.

Rapid prototyping extends this idea of questioned systems from 2D images into the creation of variable 3D products. 

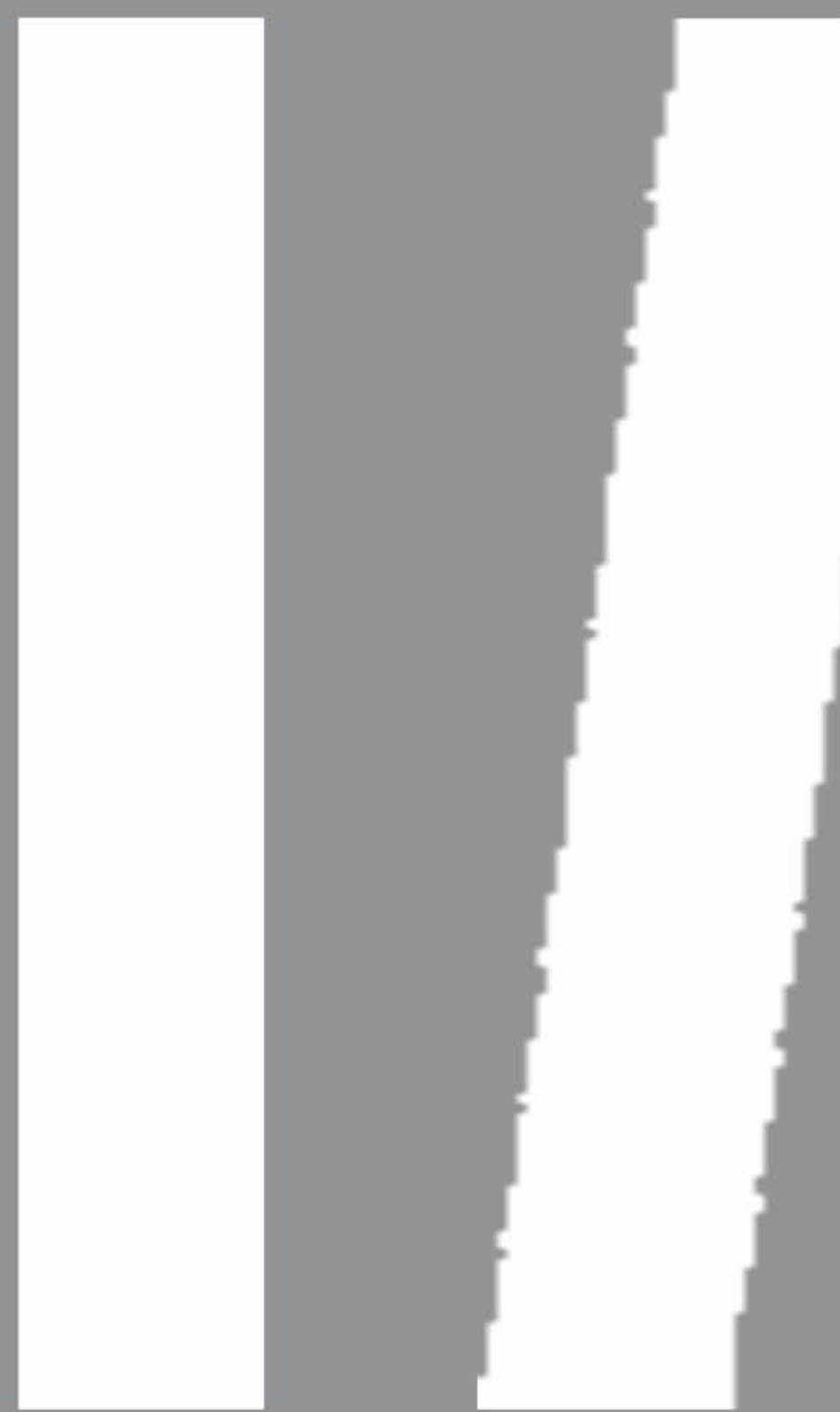

Magnified 2D Image scale 30:1

The surface of the image is pixilated due to the physical limitation of the computer screen. The variability of the edge suggests potential to create complex reactions between computer hardware 

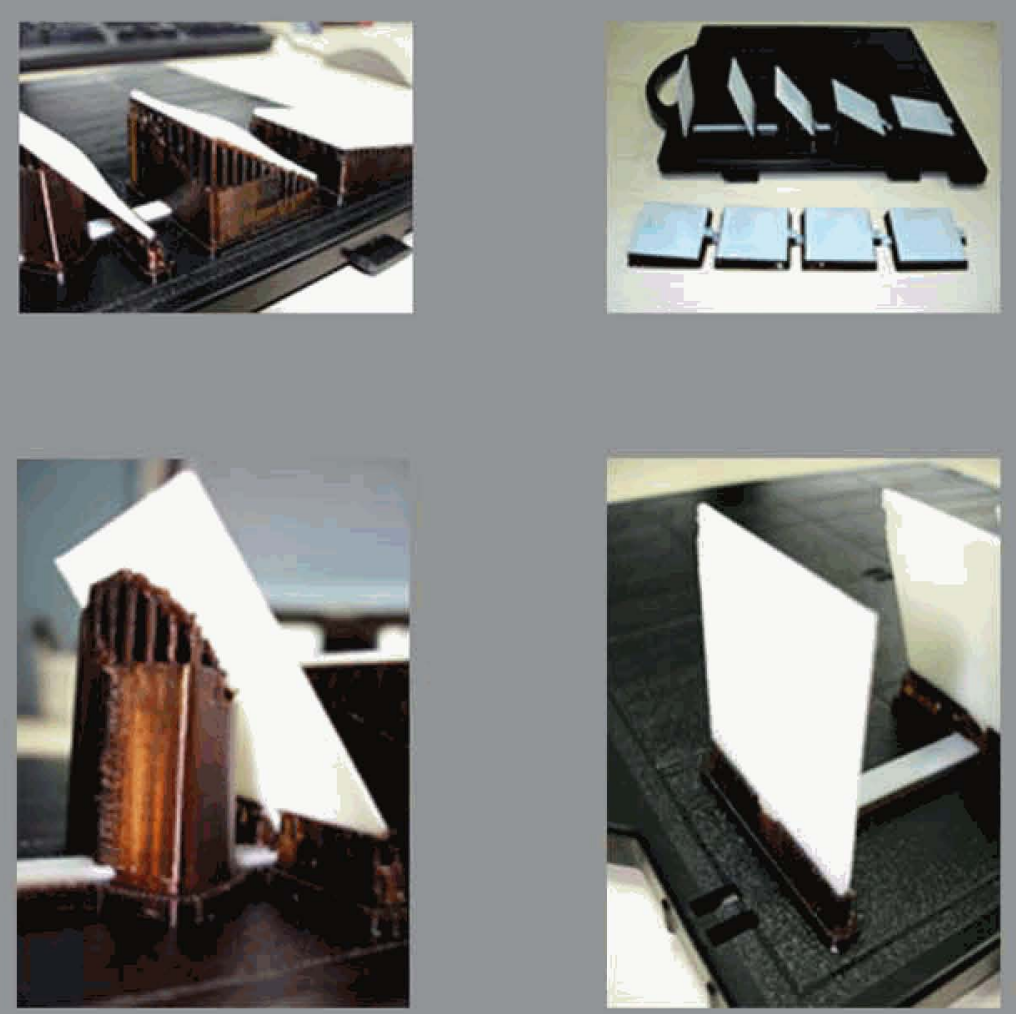

Fuse deposition modelling process (FDM)

The white plastic samples (ABS) are built on incremental angles to explore the visual effect upon the surface texture of the product

The brown material is a support structure automatically designed and generated by the FDM machines internal software. The water soluable material is separated from the product by submersion in hot water at the end of the process 


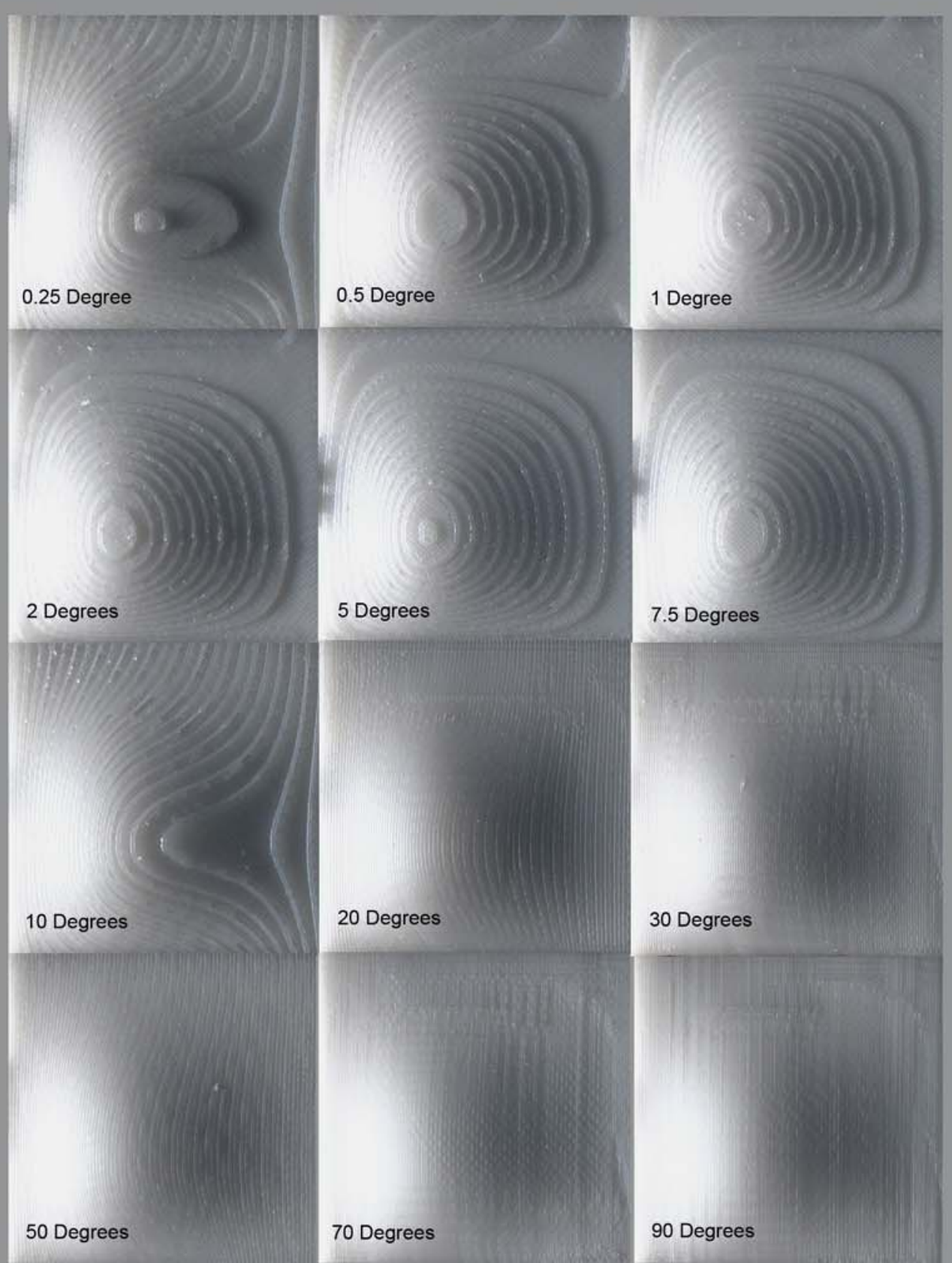

Cross lit FDM samples with varying build angles

The variation in the surface textures of these samples is dictated by the angle of the product as it is grown relative to the machine (Ref images on opposite page) starting at 0.25 degrees and progressing through to 90 degrees. The 0.25 difference between the first two samples is visually discernible which establishes the potential of an almost infinite number of unique products from one CAD file. To re-create a product both the CAD file and exact build angle are required to guarantee an identical duplicated product. 
1 degree

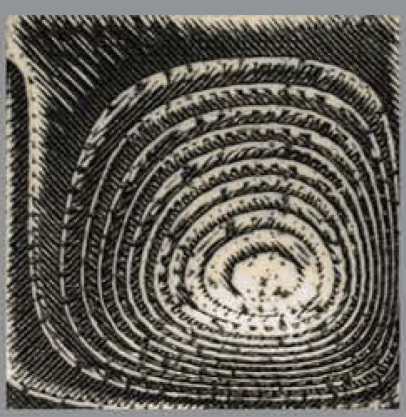

Sample \# 16
2 degrees

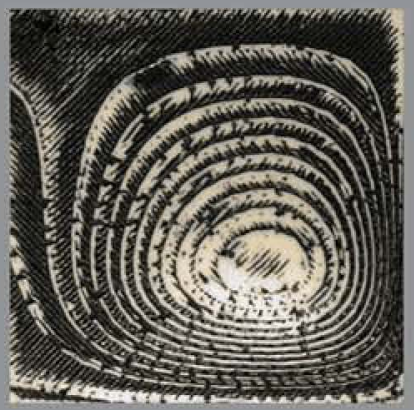

Sample \# 16.1
10 degrees

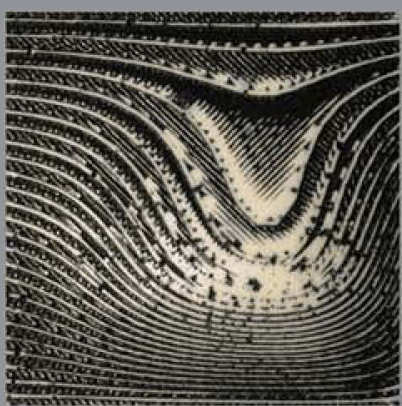

Sample \# 16.2
90 degrees

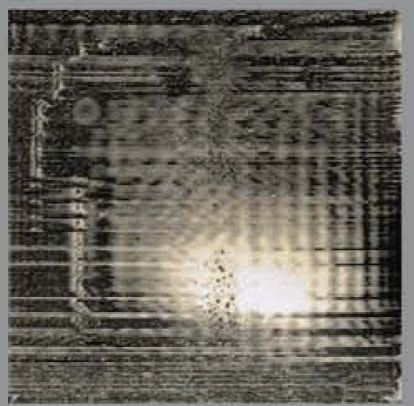

Sample \# 16.3

\subsection{Wear Revealing Detail}

By coating the FDM samples and then simulating wear through the removal of the top layer the creation detail inherent to the growing process became strongly visible. The potential of wear to act as a mechanism for highlighting initially subtle details is exposed in this experiment. The relationship between the time based process of growing these samples and the wearing away of the surface establishes a time continuum that mimics the cycle of life and death in organic entities. Each stage evolving, yet dependant and related to the previous stage 


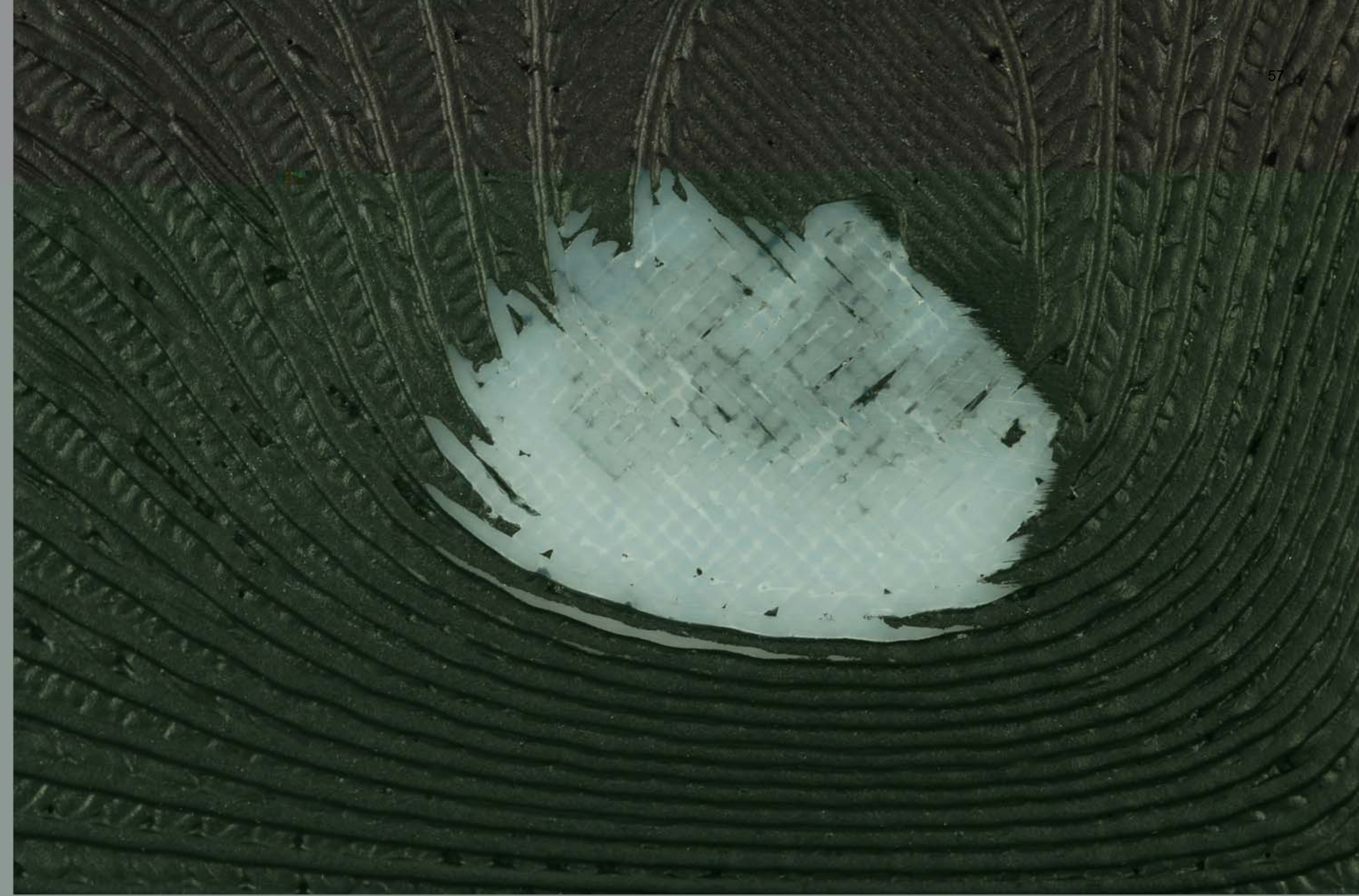

\section{External and Internal Complexity}

Sample \# 13

As the surface erodes, the complex internal structure of the woven FDM model is exposed. This establishes a fundamental difference between products that are impressions and products that have both external and internal complexity. Through this a tectonic understanding of the product's creation becomes complete and thus able to be explored below the exterior surface. 


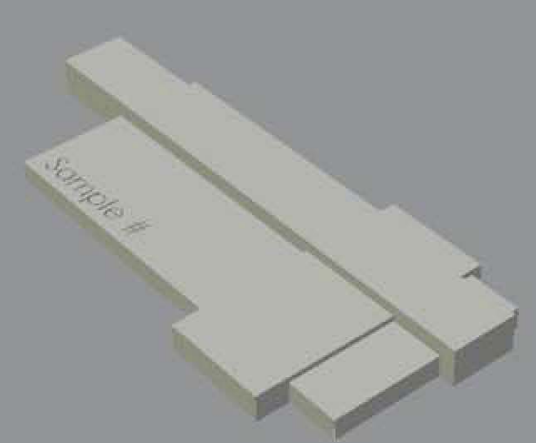

3.13 The Sensitivity of Data

It takes 189 pages of data to define this form, the change of one digit will create another object. The very mass of data suggests a sensitvity and opportunity to generate subtle variations in objects. The question this raises is at what point does the change become visually discernible and thus meaningful to people.

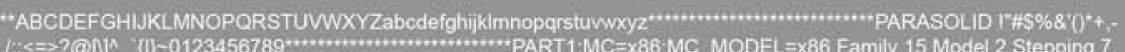

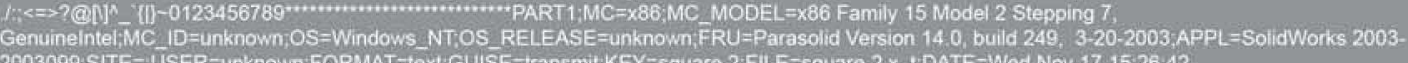

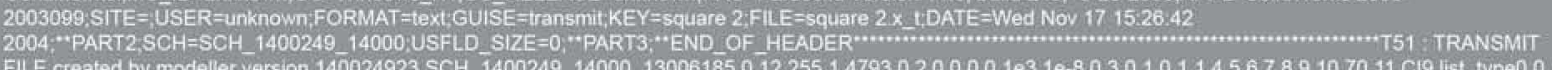

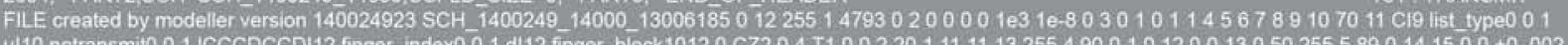
ul10 notransmito 01 l CCCCDCCDI12 finger index0 1 dil12 finger block1012 0 CZ2 04 T1 $0022011111132554900101200130502555890141500+0.002$ $001820512820512815.002-02628477564102565192558240113018$ Vic 25593700219020600118255103043021022721117255210.23 .24251026

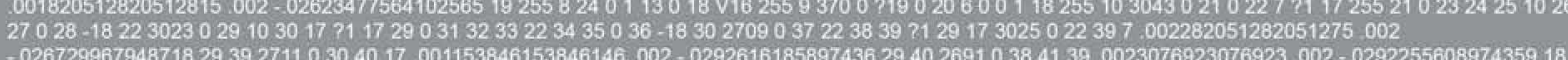

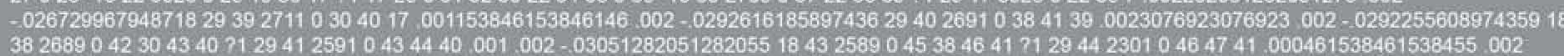
$-03238461538461541846229904843494421294722810495044.001727564102564095 .002-0322051282051282$
53.47 .001 $2947.001 .002-0349743589743591852183405449555071295315460555050.002461538461538455,002$ - 0355641025641026185515440

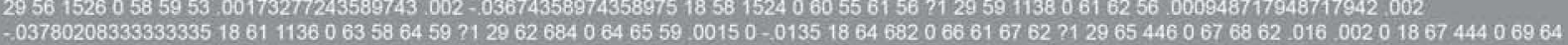
$7065 P 129684430707165.01 .002018704410726773687129714320737468 .01 .002 .0418734300757076717129744290767771 .016 .002-04$

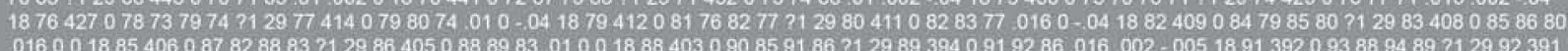
$.0160018854060878288832129864050888983010018884030908591867129893940919286 .016,002-0051891392093889489712992391$

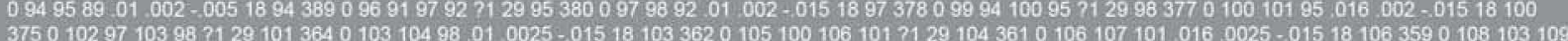
10471291073580109110104.016 .0025 - 005 $18109356011110611210771291101580112113107.018 .002-0151811215601141091151107122113$ $1550115116110.018 .002-00518115153011711211811371291161520118119113$-867361737988403e-33 002-005 1811815001201151211162129119 $1360121122116.0180-015181211340123118124119212912213301241251190180-00518124131012612112712271291251300127128122$

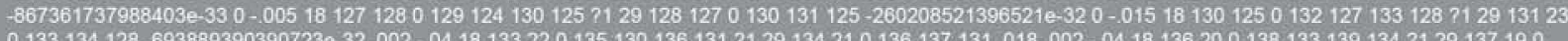

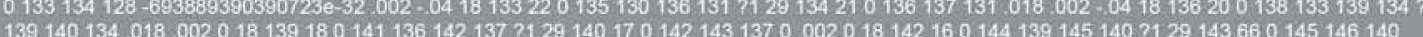

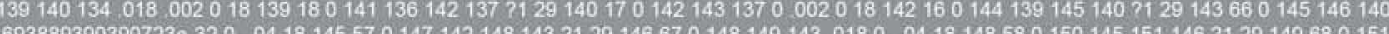

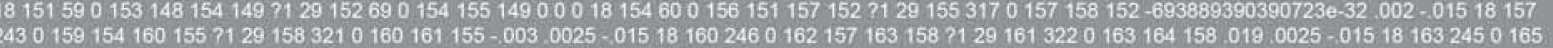

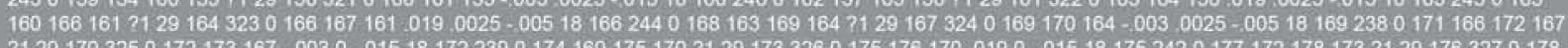

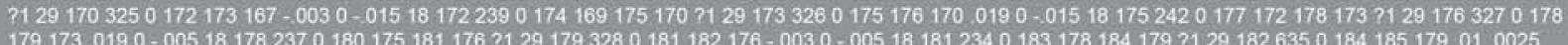
$-00518184546018618118718271291856390187188182016.0035001518187550018918419018571291886400190191185010035001518190$ 549019218719318871291916410193194188.01 .0035 -0485 $18193548019519019619121221946420196197191.0160035-0485181965430198$ 193199194712919764301992001940180001518199547020119620219711292006440202203197010.00151820253502041992052007129203 $6450205206200.010-048518205542020720220820321292066460208209203,0160-0485182085340210205211206212920910360212213206$

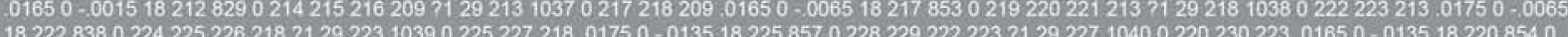
$23123221722721202301041021523322701850 \quad 0395182158550234235212230212923310420236237230 \quad 01450-0385182383330238239240$ 233712923710430241242233.01450 - $047182418580243244245237 p 12924210440244246237.01150-047182448600247248241242 ? 129246$ $10450239249242.01150-0385182398340250251236246712924910460235252246.00150$ - 03855182358350253254215249212925210480229255

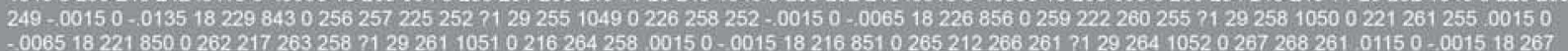
-

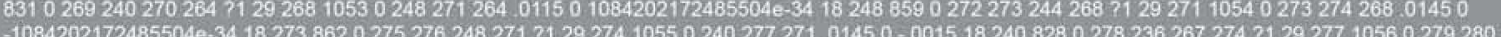

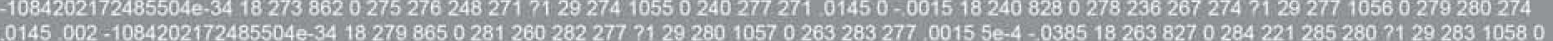
$26828628000155 e-4=0135182668420287216288283712928610590288289283-0015001-0135182888480290266291280712928910600260292$ $286.0115 .001-0065182608490293226279289212929210610257294289$ - $0015.001-0065182578390295296229292712929410620254297292$.

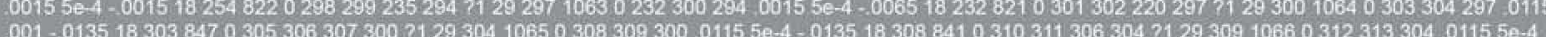
$.001-0135183038470305306307300712930410650308309300.01155 e-4-013518308841031031130630421293091068031231330401155 e-4$ $01155 e-4-0015183158300320211312316212931910690311321316$ 0115 5e-4 - 0085183118200322270308319212932110700318323319.0115 $.002-047183188630324282276321712932310710276325321.0145 .002-047182768610326318273323712932510720251327323.014556-4$ - 0385 $182518320328312239325712932710730285329325.016550 .4-0385182858250330263299327212932910740306331327.01455 e-4$ - 013518306

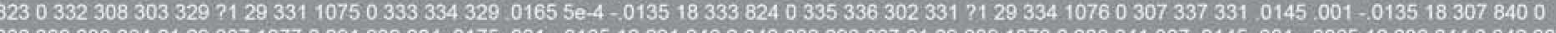
338303336334712933710770291339334.0175001 -0135 $182918460340283296337212933910780336341337.0145,001-0065183368440342307$ $333339212934110790296343339.0175,001-0065182968450344291257341212934310800270345341.01455 e-4-0065182708360346267311343$ $34910830211351347.01455 e-4-00151821181803522083153492129.35114890245353349 .000948717948717942 .0015-0378020833333333518245$ $13270354241355351712935314900356357351.0013858173076923 .002-038410256410256451835612960358359360353712935714910361362353$ $.00148717948717948 .002-038241185897435951836112980363364359357 \geqslant 12936214920365366357.001153846153846146,002$-0378020833333333355 18 $36513000367368364362712936614930369370362.001492488461538454,002-0374102564102564518369130203713723683667129370 \quad 4940373$ -03784254807692311837713060379380376374712937814960381382374.00228004807692307 .002 . 0381702724358975183811308038338438037871

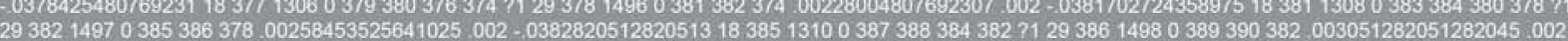
-03778926282051285 $1838913120301392388386212939014990393394386.00271274038461538002-03725641025641031839313140395396392390$

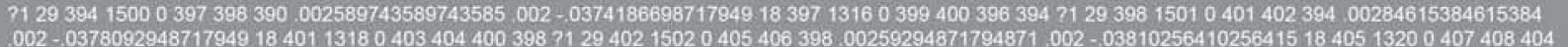

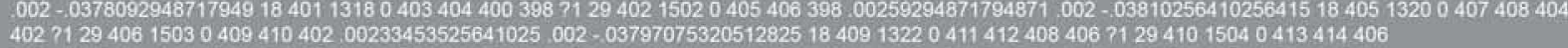

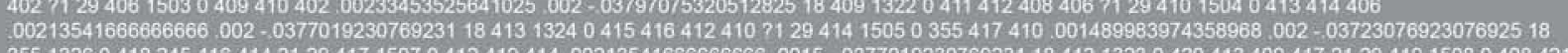
$35513260418245416414212941715070412419414.00213541666666666 .0015-03770192307692311841213230420413409417 ? 12941915080408421$ $417.00233453525641025 .0015-037970753205128251840813210.422409405419 ? 12942115090404423419.00259294871794871 .0015$ 


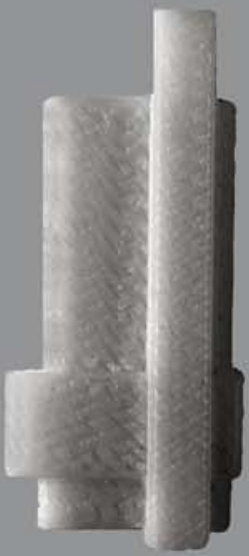

These two samples were grown on a 'Fused deposition modeling' (FDM) machine that uses a fine bead of plastic $(0.3 \mathrm{~mm})$ similar to a hot melt glue gun. The angle at which they were built was modified to explore the resulting changes in surface pattern.

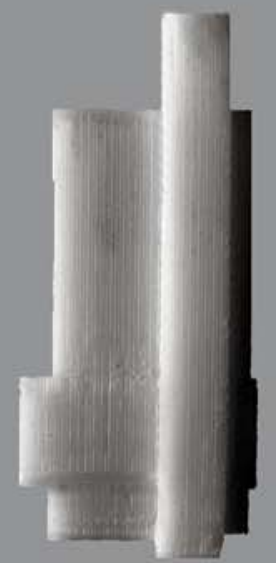

\subsection{Virtual and Physical Sites}

In addition to the variations offered by manipulating data are the variations offered by manipulating the physical 3D printing machines themselves. As these processes are relatively new and unfamiliar, there is a tendency to group all 3D printing systems together even though each system offers distinct material and tectonic qualities. Samples using the same CAD file were produced on two different systems to allow an aesthetic understanding of the products in a physical rather than virtual context. Differentiation between virtual and physical is critical because these products exist equally in both yet hold distinctly different qualities in each. The understanding generated through this research about products and materials as they transform through human contact holds equal potential to influence the creation of products in both the physical and virtual worlds. The need to create evidence of a person's existence in the virtual world eventually might exceed the requirements of the physical world as people desire and are required to spend more time occupying virtual environments. 


\subsection{Re-construction through Wear}

The manufacture of the centrifugal casting mould required heat and pressure resulting in the destruction of the original rapid prototype samples. The combination of high cost and technological reverence given to rapid protoypes has kept our aesthetic understanding of them largely platonic. A primary motivation in molding multiple samples was to gain the freedom to explore below the surface to understand the aesthetic transitions they underwent as they became worn. Continuing the wear process beyond reasonable limits resulted in the deterioration of the surfaces that extended into the destruction or re-construction of the form. 


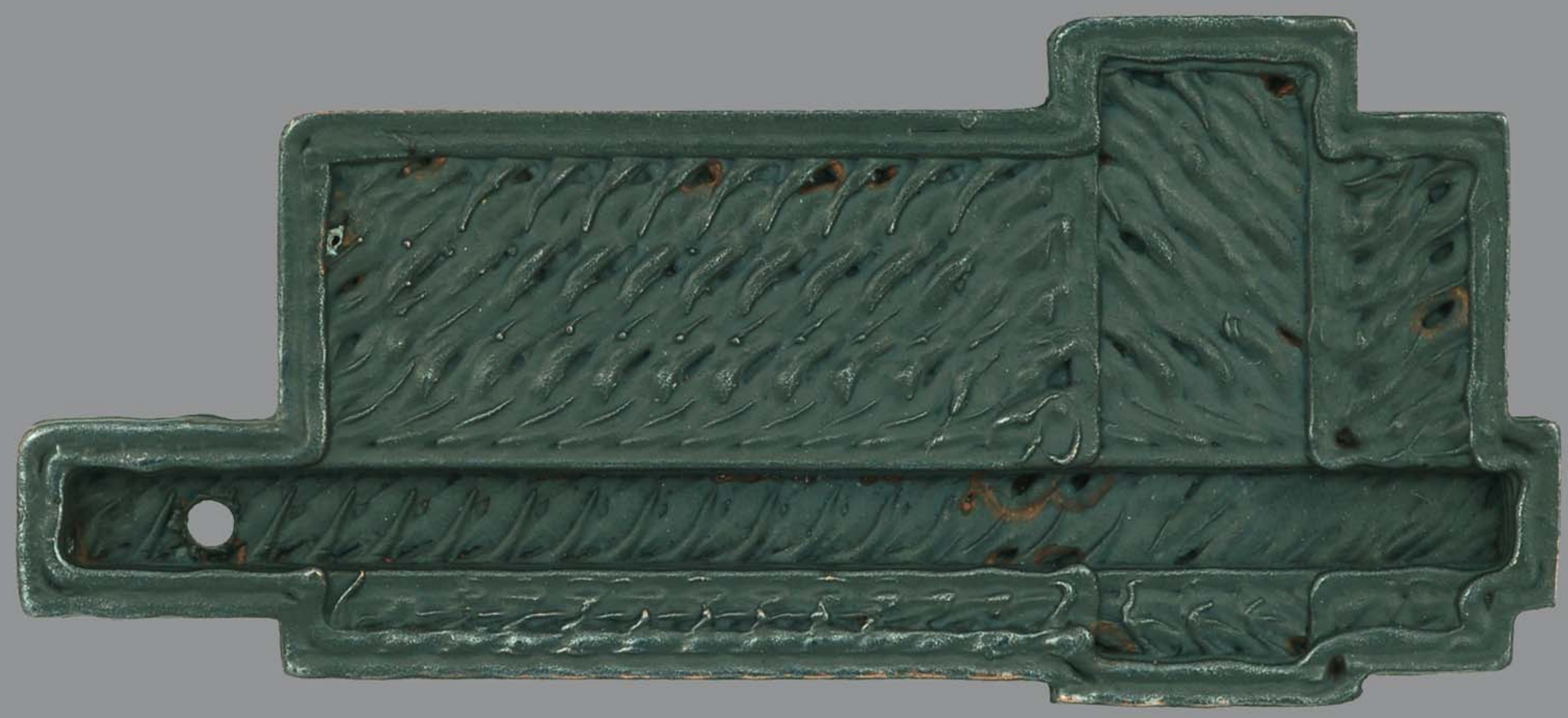

Related Materials

Sample \# 168

The dull grey oxidised copper layer over this product conceals a surprising relationship to the bright polished pure copper underneath. It is derived from the same base element yet the stark contrast between the surfaces attests to the potential of time to transform the aesthetic qualities of materials. Unlike an unrelated painted surface, clues of the connection are evident in small areas of bright copper left visible on the exterior surface. 


\subsection{Conclusion}

As this incarnation nears it end, the product is now layered in visual evidence of its distinct history. The exposed skin presented to the outside world evidences the effects of a lifetime. The making process manifests from an idea, a sketch, a virtual model, a 3D print, a generic moulding and eventually into a unique artifact. It has travelled along a path into the future through the layers of its past. Its worn surfaces record the relationship between the person who created it and the person who continued the creation through use.

As the instigator of the journey, the notion of focusing all of the design vision on the product when new seems a denial of the responsibility associated with the act of creation, to give an ongoing life to the product. 


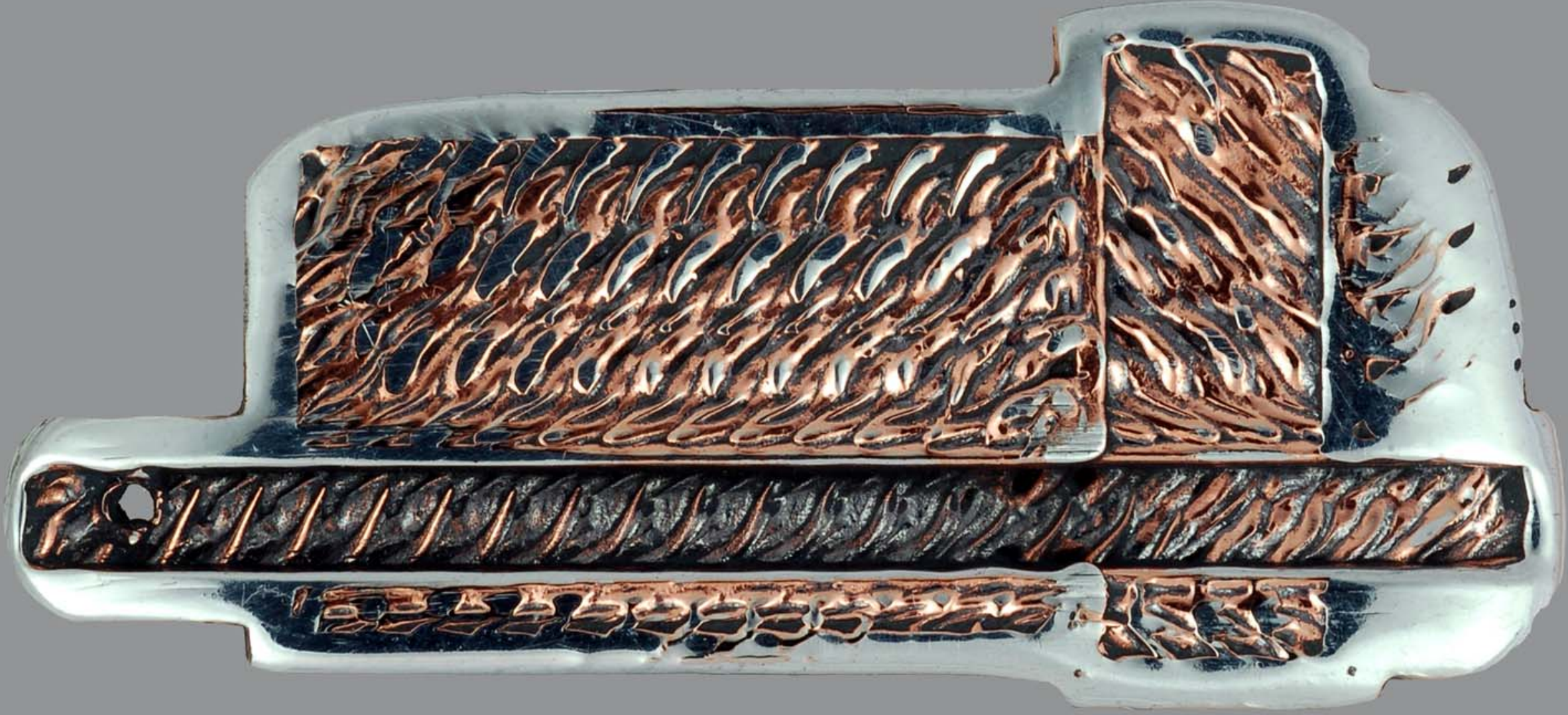

Manufactured and Personal Artifact

Sample \# 174

As the oxidisation wears away the feathering between the materials reveals both contrast and connection. The form and texture of the product disproportionately removes and retains the layers allowing new and old surfaces to exist similtaneously. 

4. Envisaging the future 
This chapter explores the need and emerging potential to envisage the future

\subsection{Physical and Digital Simulation}

The industrial design process inherently involves the envisaging of future scenarios and opportunities. Traditionally this has been achieved through hand drawings and physical models. With the specialised knowledge of the industrial designer these can be read and understood as tangible and complete products. The aesthetic focus of this research required a simulation process capable of compressing time and amplifying the visual complexities of material and form interactions.

While physical simulation techniques have been able to reveal aesthetic qualities generated through wear, the time and effort required to do so limits the commercial application of the research method. As the capacity of computers to predict and visualise continues to increase, 4D programs will emerge to allow lucid visions into the future. This will expand the design process where the actions of today are informed by an understanding of likely reactions in the future. 3D models will extend into $4 \mathrm{D}$ simulations revealing the lifecycle of a product from creation and use to disposal. $\mathrm{A} 4 \mathrm{D}$ refinement of the product will allow designers to increase or reduce the speed of transition through manipulation of the products form, materials and wear action thereby designing an artifact that is considered and connected with time.

Embedded in a monitored space and time and wrapped in a haze of process, no object stands alone; it is not a static thing, but a shaped thing. Thanks to improved capacities of instrumentation, things are no longer perceived as static-they move along a clocked trajectory from nonexistance to post-existence. 14 


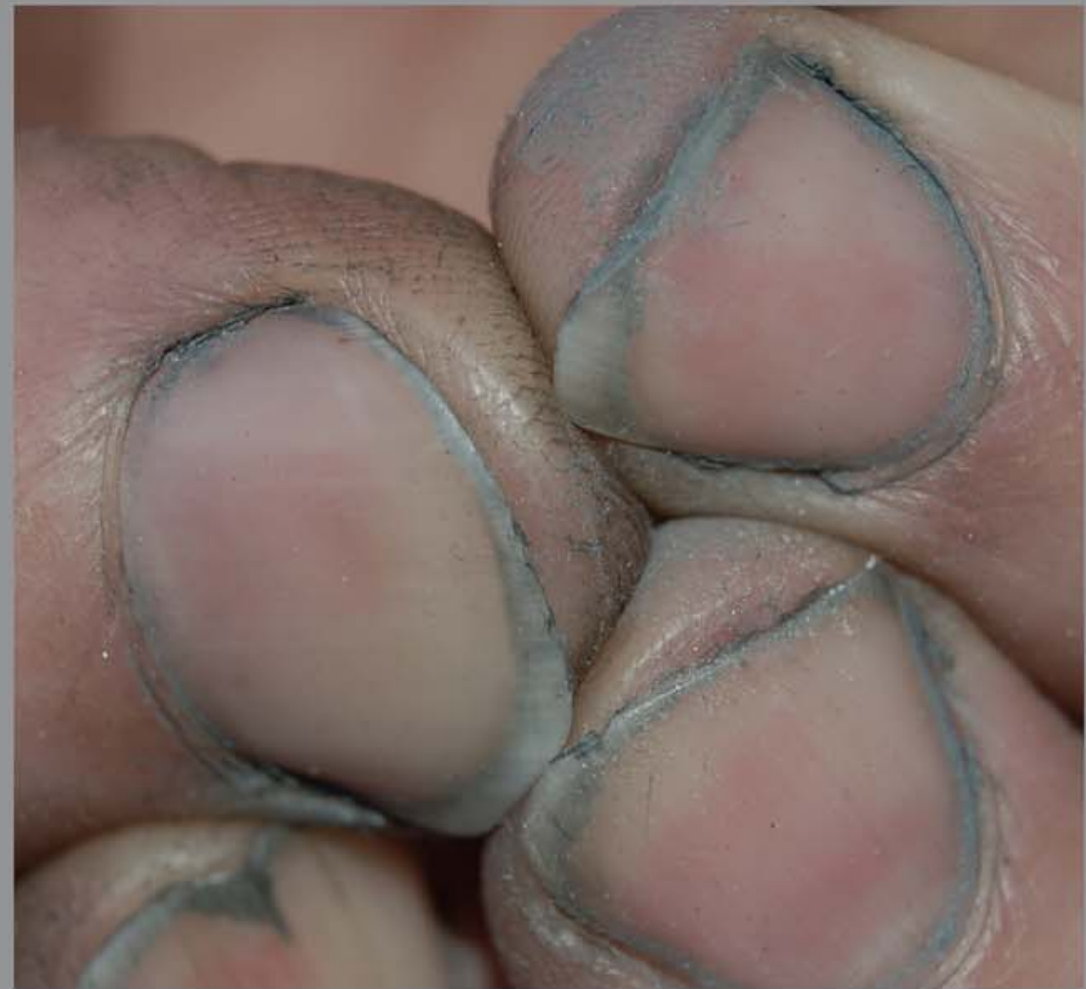

Finger nails worn during physical modeling

\section{Physical simulation}

The actual physical processes used to simulate wear in the products required extensive amounts of time and skill that would restrict the commercial applications of the research technique. For these reasons $4 \mathrm{D}$ simulation will need to become standard practice once computers have developed that allow fast, accurate and visually detailed simulations. 


\subsection{Informed Consent}

A series of symbols derived from hallmarks engraved into the back of the product establish information specific to the company and design team responsible for the products creation.

A material mark exposes the contrasting layers used in the product's construction, offering information about its history and its future. The deliberate removal of small sections of each layer reveals the designer's intention that the product is expected to change through use, yet the process of envisaging the transformation remains in the imagination of the person considering the relationship. As the layers are revealed, the relationship continues to be transformed and refreshed through a diverse range of receding and emerging aesthetic qualities. The traditional association between wear and the destruction of the designers vision is now understandable as an act of discovery and construction within a shared vision.

Critical to this is the clear communication of the designer's intent for the product to change to allow a process of radical change to be one of informed consent. 


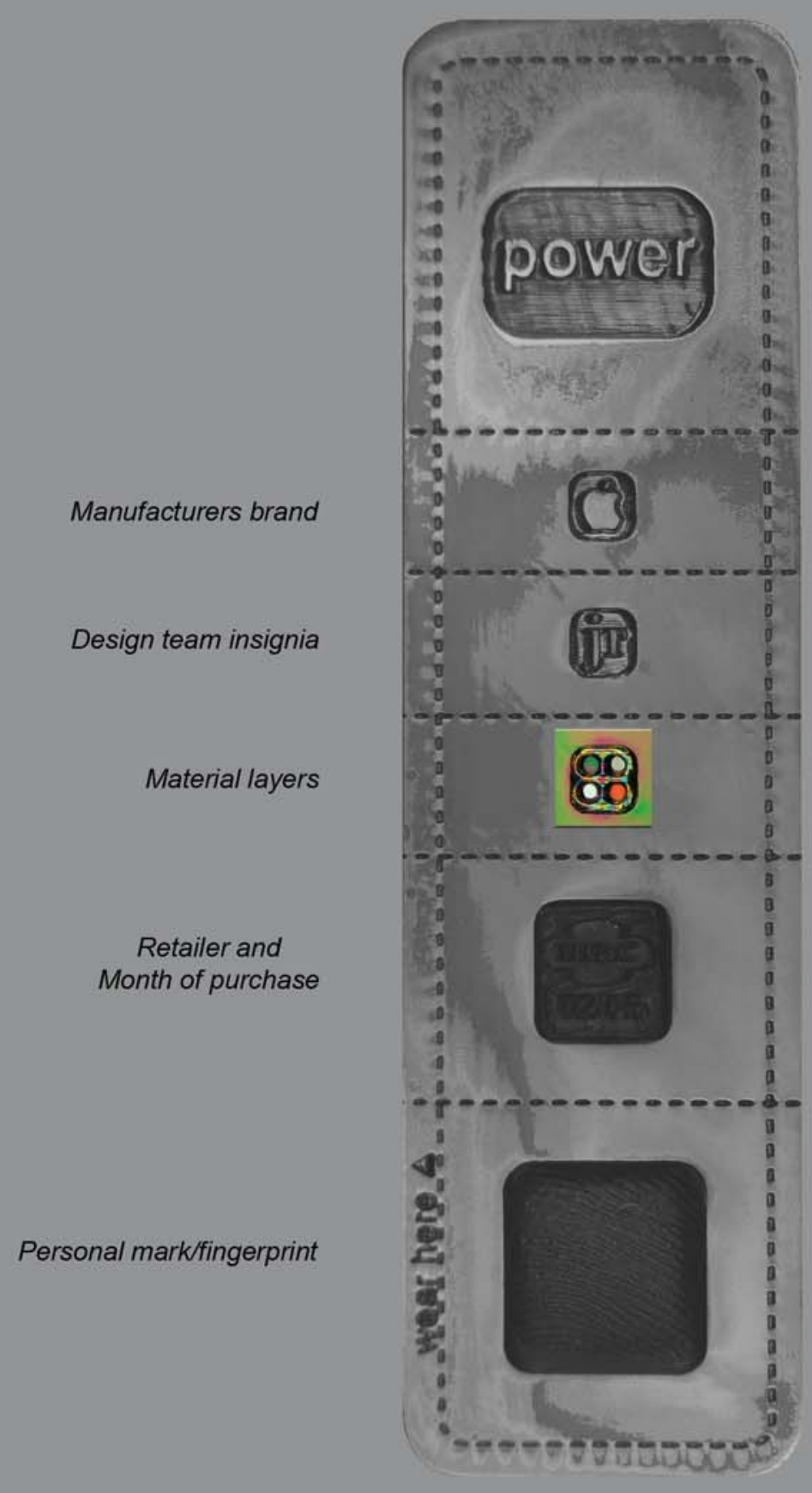

Rear

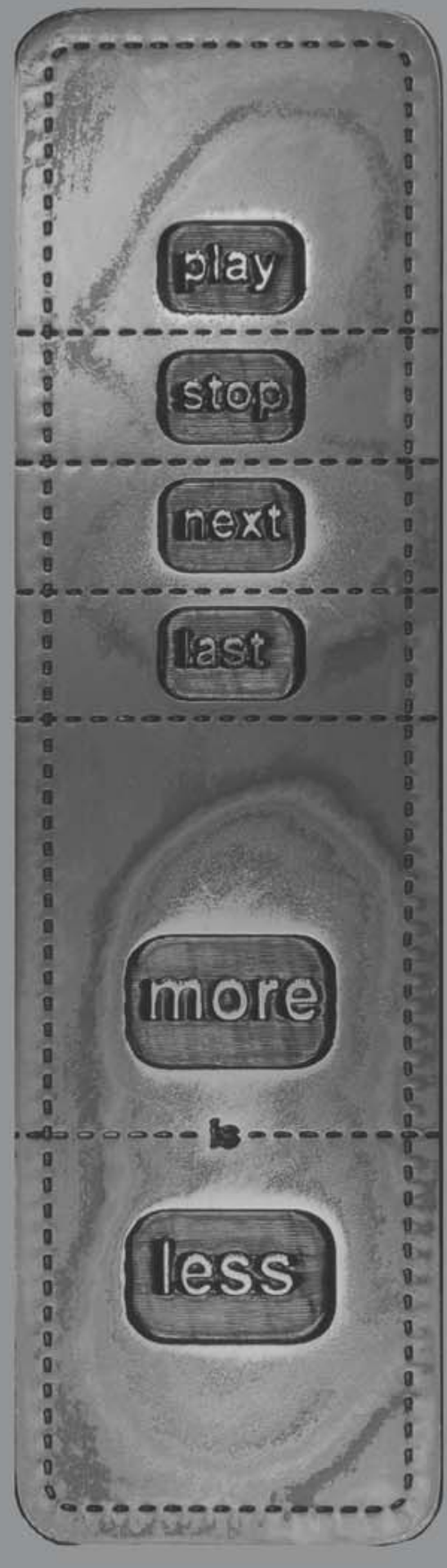

Beneath the Skin

Small areas of the products skin are removed to allow a preview into the

layers beneath, offering a suggestion of how the product might change through use.

Sample \# 262 


\subsection{Connecting People}

Traditional media associated with consumer electronics including glossy magazines and television advertisements focus on the image of the product when it is new. The showrooms that retail the product naturally have a similar focus when promoting products. The remaining traditional and non-commercial communication channels of direct contact between family and friends have become less relevant as the diversity of products on offer has increased, reducing the likelihood of direct personal experience.

The rapidly increasing communication platform of the internet has introduced yet another medium that is connecting people who have already used or bought a product with those who are considering doing so. Thus the experience people have using a product is becoming increasingly important in the continuous selling of products. This shift means that the performance of the product throughout its life, beyond the showroom is becoming more important to manufacturers and retailers and thus requires greater consideration when the product is designed.

Information associated with the business is also increasingly able to be shared electronically, allowing user scrutiny that extends beyond the company's own promotional information. This shared information encourages manufacturers to monitor all stages of the product's life from creation through to disposal. Manufacturers are increasingly aware that this information is becoming available in showrooms through hand held internet linked devices. When the person is deciding which brand to support, they will do so with the knowledge of how the business operates and the values it holds.

THE ONLY SANE WAY OUT OF A TECHNOSOCIETYIS THROUGH IT. INTO A NEWER ONE THAT KNOWS EVERYTHING THE OLDER ONE KNEW. AND KNOWS ENOUGH NEW THINGS TO DAZZLE AND DOMINATE THE DENIZENS OF THE OLDER ORDER. THAT MEANS BRINGING MORE ATTENTION AND ANALYSIS TO BEAR ON OBJECTS THAN THEY HAVE UNDERGONE 15 


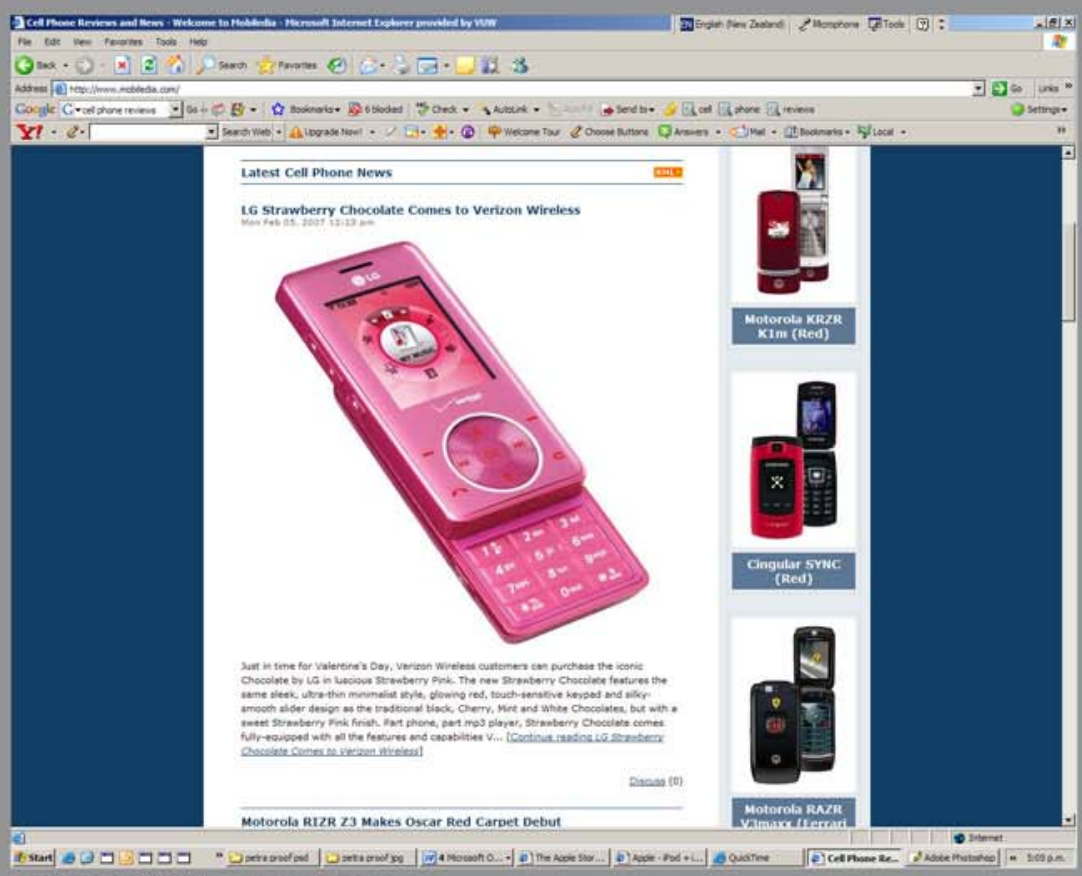

Image source: http://mww.mobiledia.com

\section{Connecting People}

The use of the internet by experts and amateurs to publish their experience and opinions about products creates a connection between people that will require manufacturers to focus beyond how the products appears in the showroom to how it performs throughout its life. 


\subsection{Informed Consumption}

The emergence of visualising tools capable of creating accurate and visually sophisticated simulations will extend beyond the designer to the consumer. As products become more sophisticated so, too, will the visualising tools which allows a balanced equation of complexity and comprehension to evolve. The ability to foresee how a product will change allows greater comprehension by the person purchasing the product of their needs and aspirations. Disposable products could be evaluated for their environmental performance, including the infrastructure to recycle or dispose of them. Durable products could be evaluated to reveal their transformation from generic products into personal artifacts. Manufacturers could use visualising software to extend the retail experience and justify a price premium on products for aesthetic qualities that are yet to appear in the

$$
\text { product. }
$$

A process of education will occur for people making, selling, buying and using products.

Embedded in a monitored space and time and wrapped in a haze of process, no object stands alone; it is not a static thing, but a shaped thing. Thanks to improved capacities of instrumentation, things are no longer perceived as static-they move along a clocked trajectory from nonexistance to post-existence. 16 


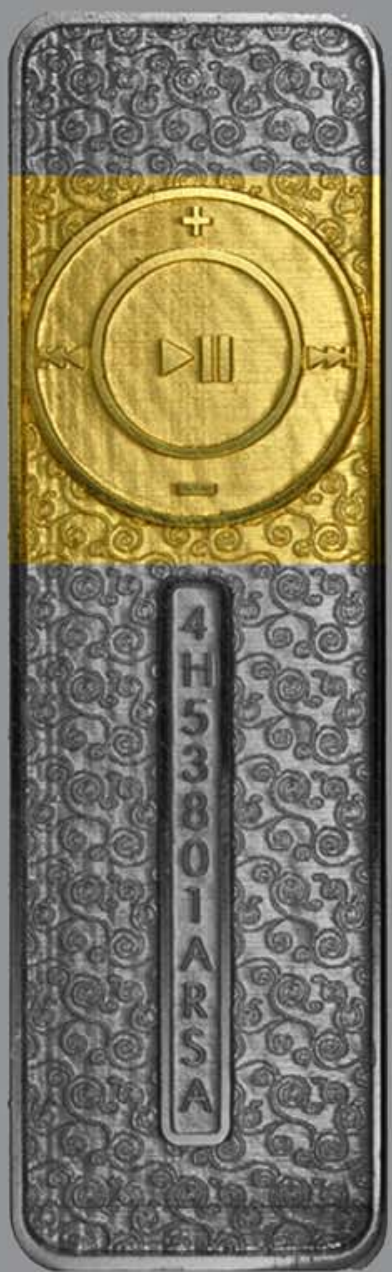

Too new

Sample \# 243

Without oxidisation this product is visually generic and disconnected from time. Its polished surfaces can only be diminished through contact and for that reason it does not have a sustainable future and should not be sold, yet.

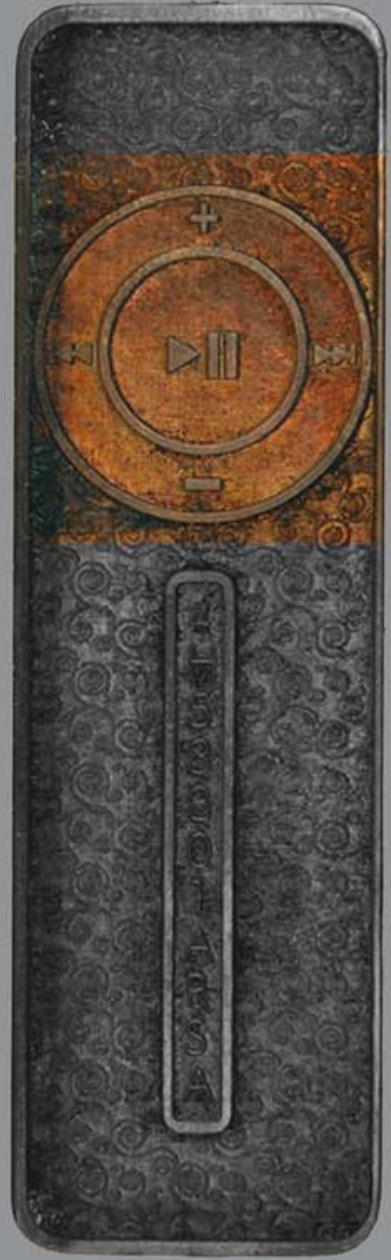

New

Sample \#244

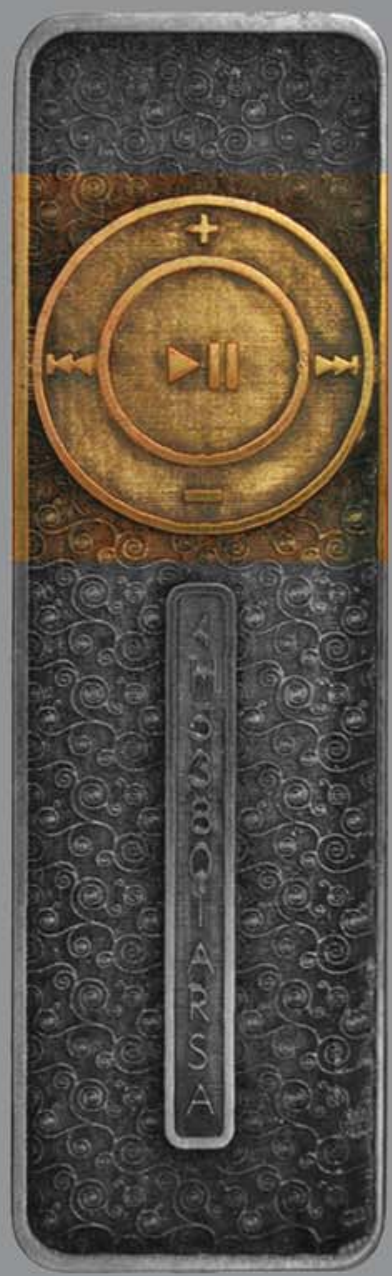

Worn in

Sample \#245
The product is designed to be sold with a unique surface patina that is polished away through use. This deliberate transition allows the person using the product to increase not diminish its aesthetic qualities. The wear will occur at only the point of surface contact and will therefore be unique to that user. 


\subsection{Conclusion}

The challenge of $4 \mathrm{D}$ design is to comprehend objects not as singular static entities but as connected and continual transformations. The glossy product images of today will have no relevance in the emerging world of future visualising software and physics calculated in real time. Once

designers embrace the tools to work in $4 \mathrm{D}$, they will use their inherently inquisitive process to explore and expand both their own creative boundaries and the boundaries of this infinite time rich world. Anatomical models will be merged with product models and interface software will scribe the choreography. The form of the product will no longer be understood as an arbitrary shape, it will be a readable map of energy that focuses and dissipates the precious wear resource. Materials will move from being facades trying to maintain their deceptions to breathing, reactive entities responding to the atmosphere, human sweat and all of the layers above and below them. The skins of the product and the way they sit, merge and erode together will be manipulated and refined, not for the showroom but for the product's second birthday. As designers become more confident to

work with the dimension of time, they will explore products that last for just a few remarkable seconds or endure as inter-generational heirlooms.

Designers will loose their apprehension of the effects of time and will reflect on contemporary philosophies which focus on the resistance or denial of time as inherently futile and disconnected with the rhythm of the world and everything in it

Through our products we will once again become connected to time from a known and vibrant history to an equally known and interactive future. 

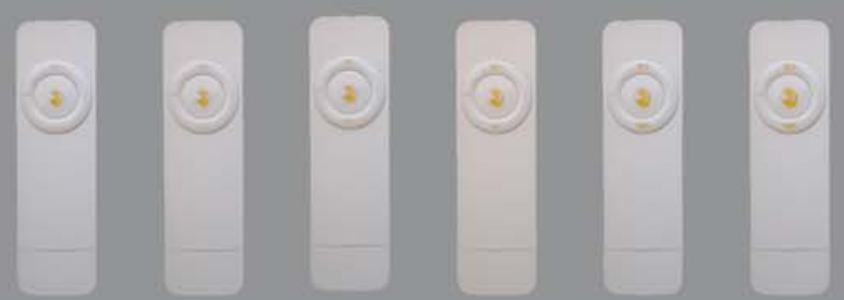

Video Images
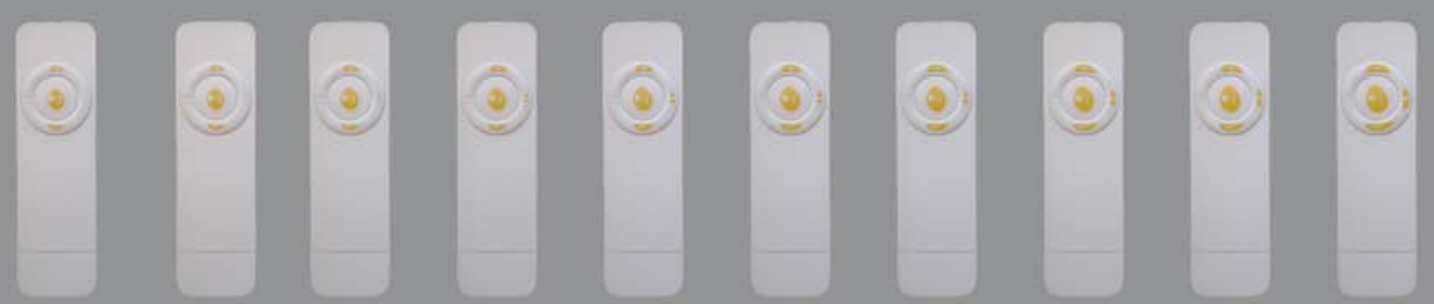

As products become understood from a 4D perspective, the potential process of transformation can be simulated through animation. This

compression of time allows an

understanding of the appearence of the product in one, two or ten years in the future.

This process was simulated using physical models (based on the Apple Shuffle) that were incrementally

photographed during the wear

process. These images were then

sequenced to create an animation.

Ref enclosed DVD
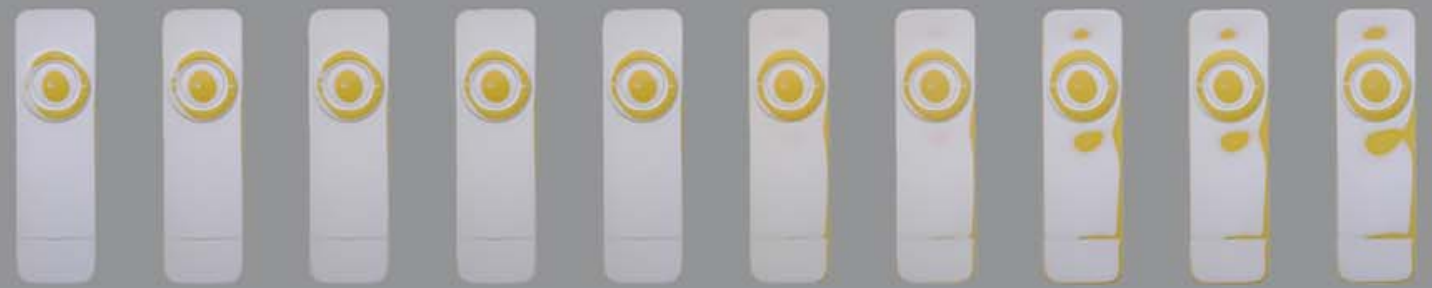

Sample \# 4.4 (destroyed during simulation) 
5.0 Design Experiments 


\section{Design Experiments}

The primary methods used to undertake this design research by composition were experimentation and observation.

The experiments required the creation of physical samples and physical wear simulation techniques. The interaction between these formed the basis of the design experimentation process. A balance was needed to control the experiments to allow the generation of discernable and attributable information without restricting the potential to reveal unforeseeable yet valuable information. On the conclusion of each experiment, observation of the results allowed the creation of the questions to be answered in the next. From this a self-generating research process evolved that explored, revealed and substantiated the issues.

The initial pure design experiments reduced the number of variables and issues explored to allow an understanding of specific factors. As these factors were defined, they were then synthesized to allow the creation of applied design experiments that offered a contextual and directly tangible perspective. The results of the physical experiments and their implications were explored through hypothetical scenarios explored as design fiction (ref chapters 3 \& 4).

To create a dynamic understanding of products as they evolved through time, multiple images and physical samples were produced Animation of the images allowed the effects of time to become rapidly visible and thus workable (ref enclosed CD). The creation of multiple physical examples at various stages of wear allowed the retention of 3D information that spanned a 4D time frame. 
5.1 Time and Design 
Experiment: \# 1

Primary design issue: The importance and potential of time as a fundamental design issue 


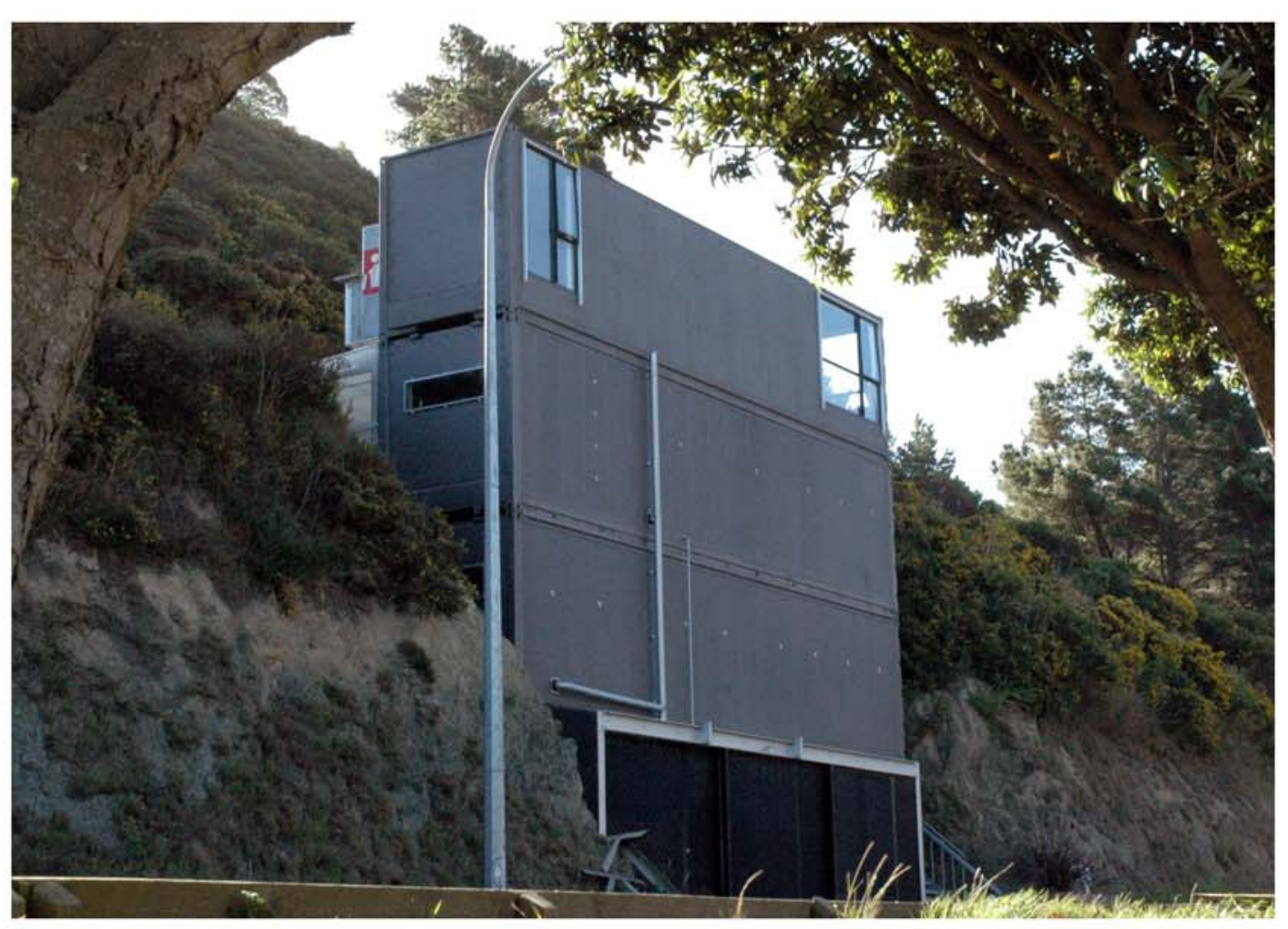

House Exterior 173 Happy Valley Road 


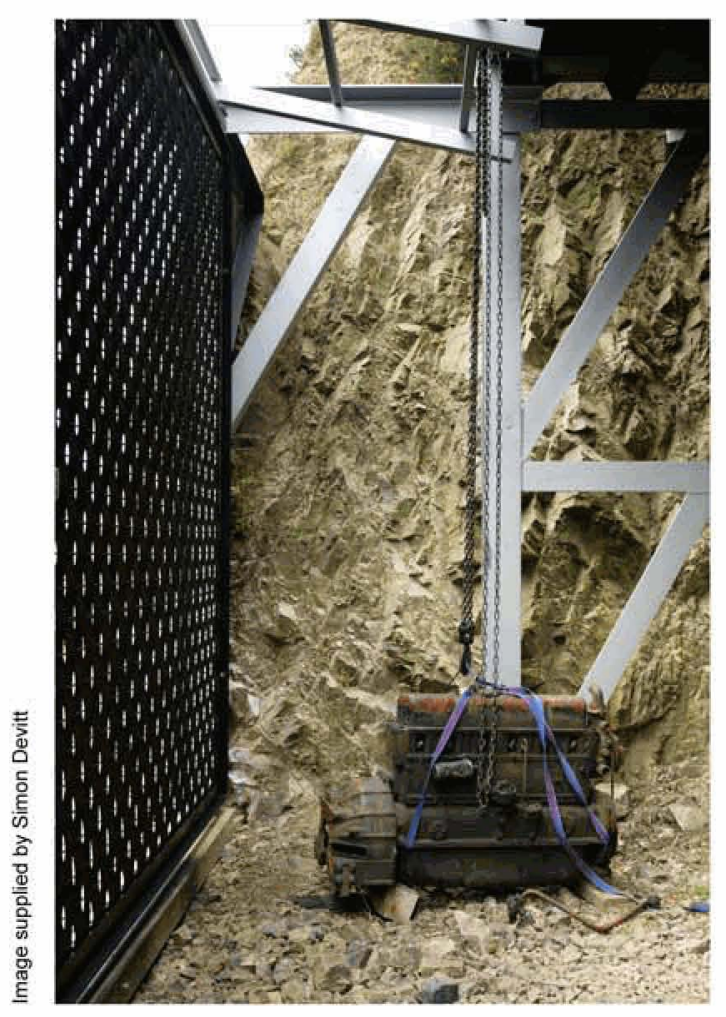

Ground level Garage

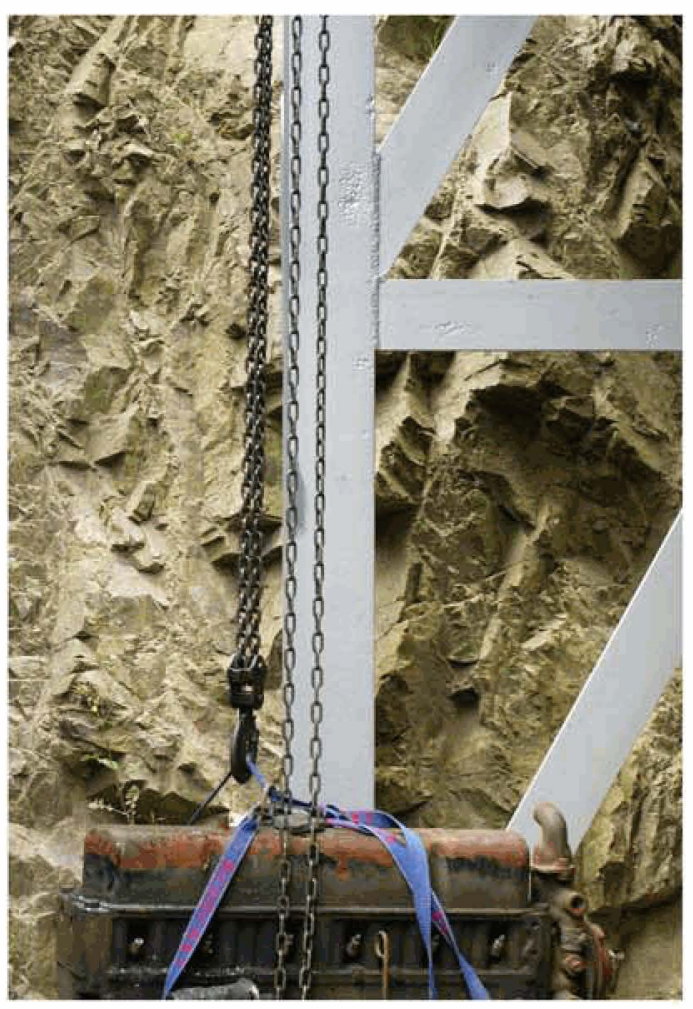

Detail of sandblasted and painted steel frame with visible pit marks caused by earlier rust. 


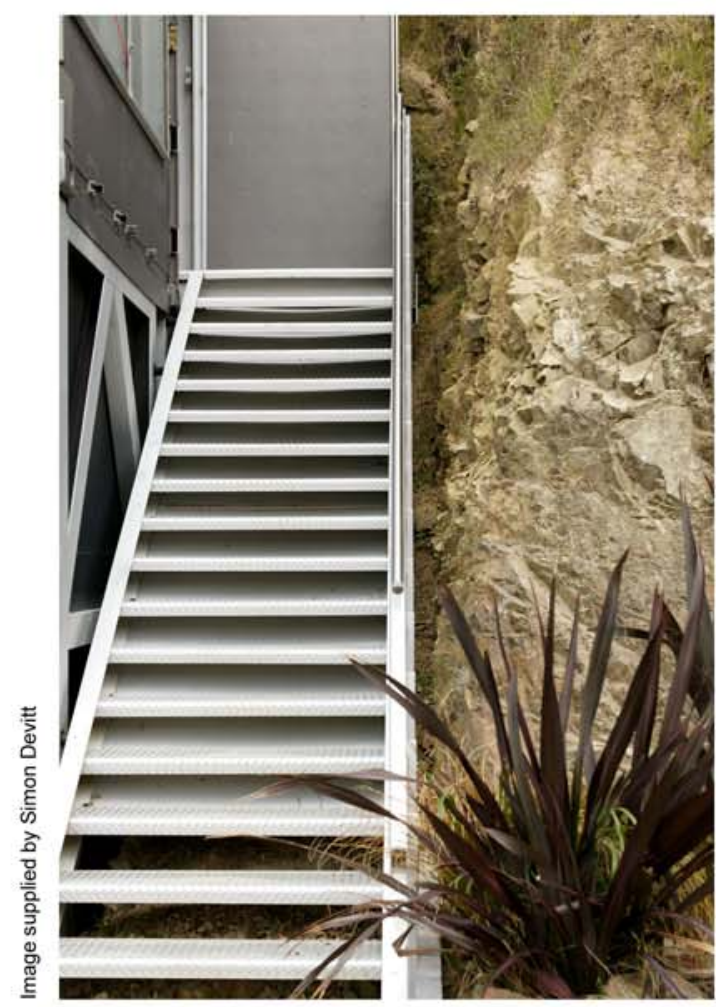

Ground level Entrance stairs

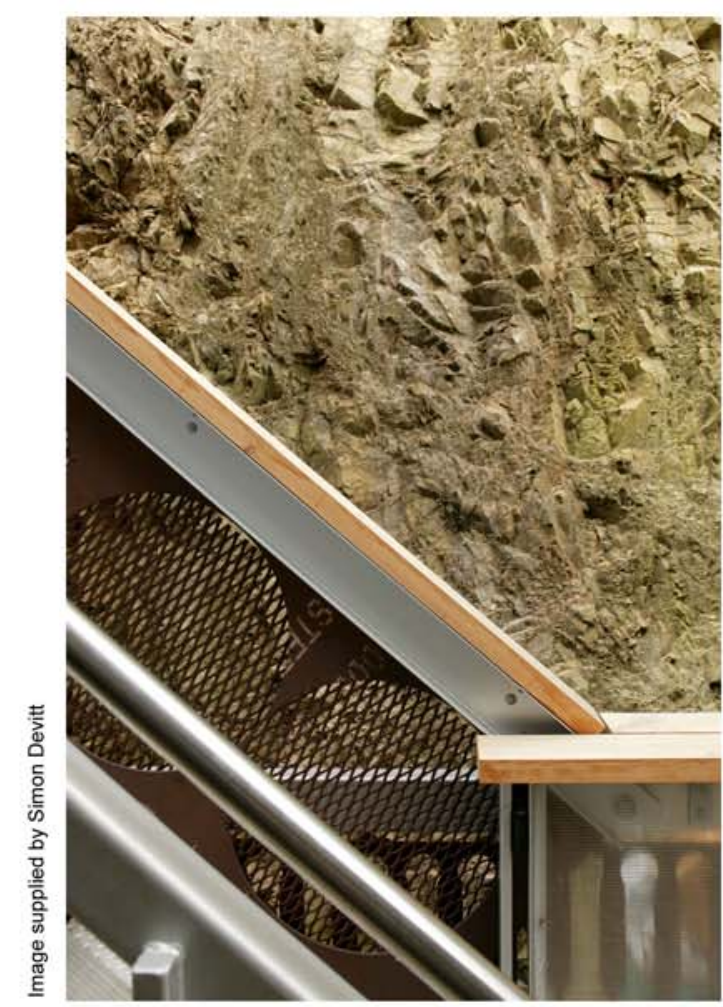

Level 1 Stairs to level 2 


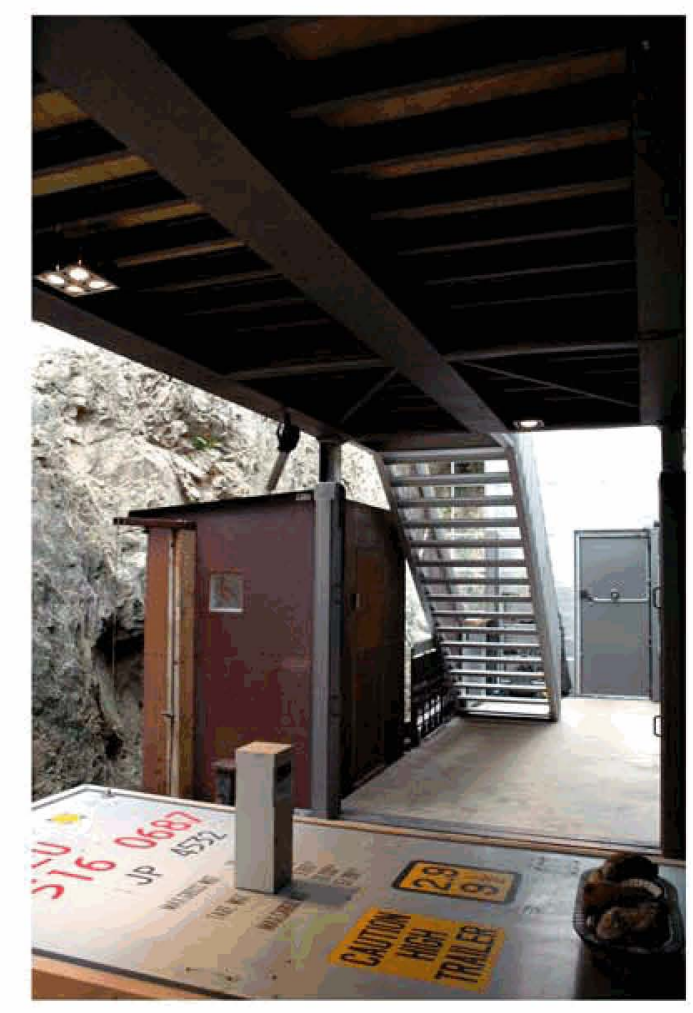

Level 1 Covered exterior deck

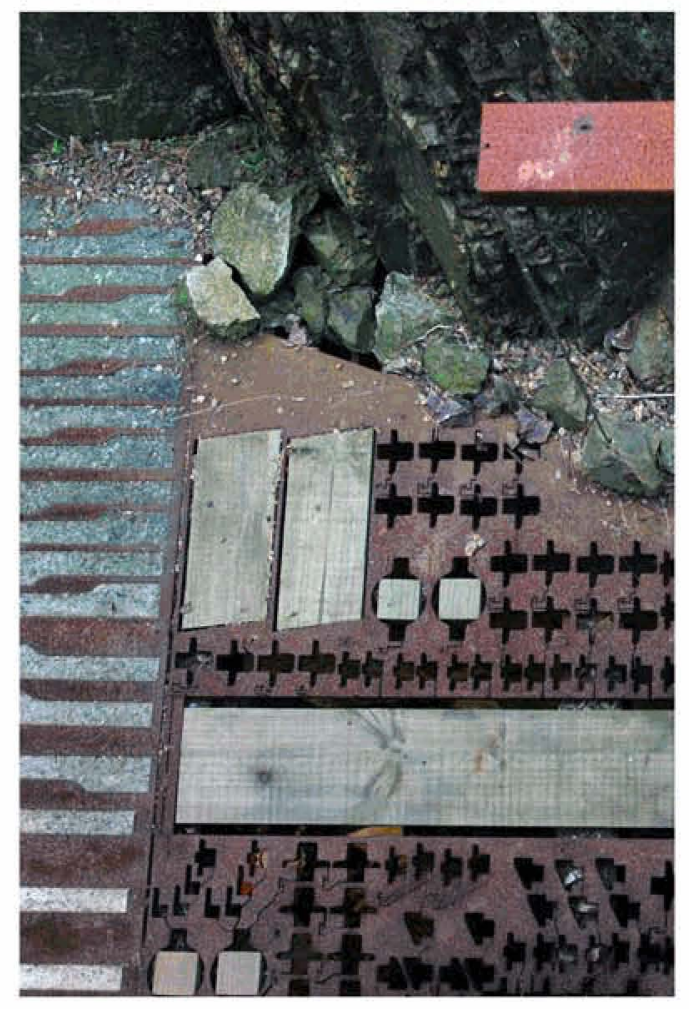

Level 1 Oxidised steel floor 

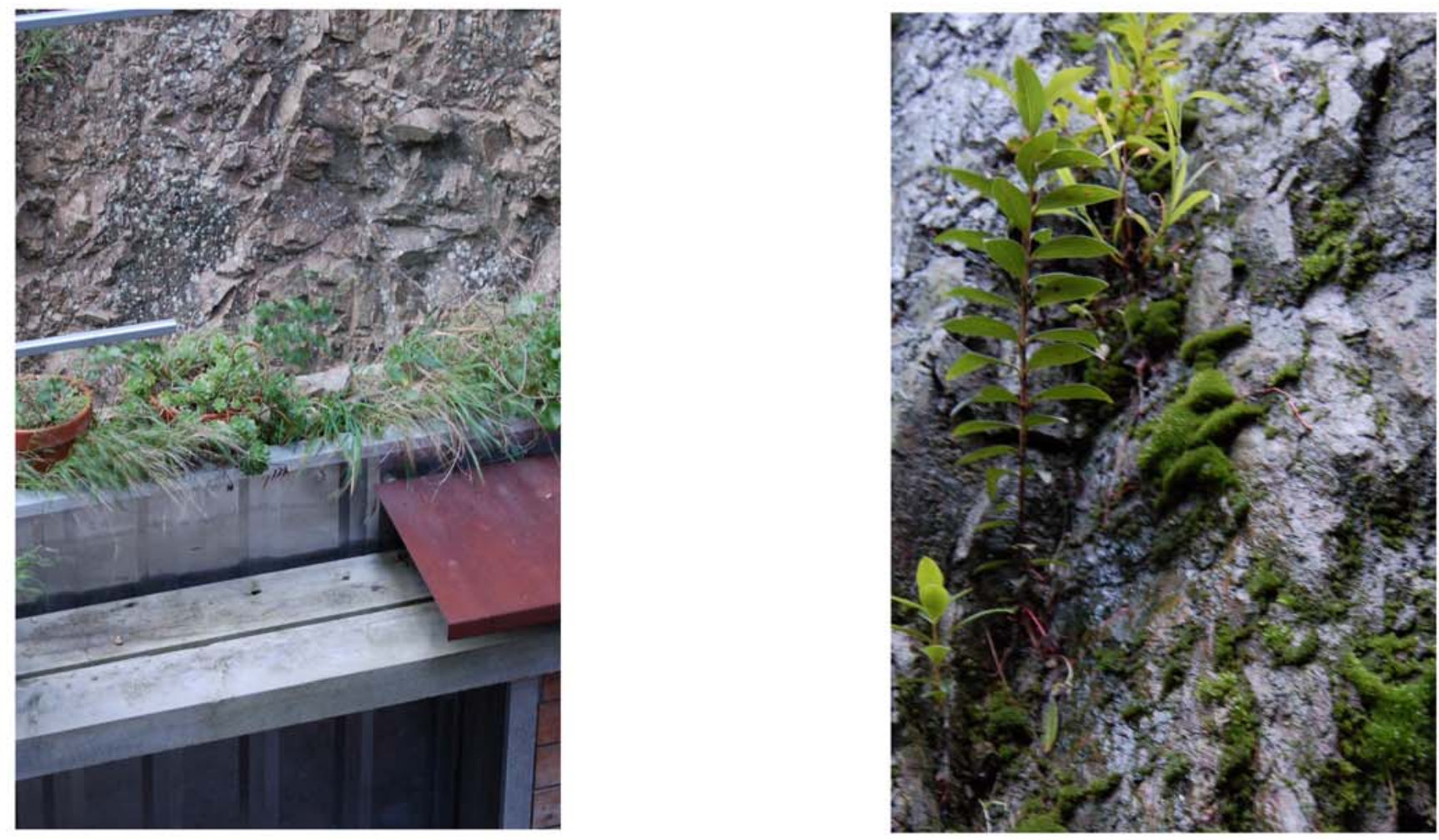

Level 1 Constructed herb garden

Level 1 Self seeded plants 


\section{Observations}

The legal requirement to prove the future durability of the building (50 years) showed a design understanding and expectation to foresee long-term performance beyond the parameters of the Industrial Design profession. The use of uncoated steel that was left to weather required a calculation of the expected annual destruction of that specific material on that specific site. This confirmed the importance of site specific information to accurately predict future reactions. These predictions were solely based on the physical performance of the particular material yet the potential and need to foresee aesthetic qualities seemed equally compelling and significant.

A consistent metallic paint applied over the building exterior concealed evidence of the diverse histories of the old products, used to create the new design composition. While the surface was consistent, marks left in the steel created by rust revealed a complex history. As the connection

between the building and the land became more intimate, the dissimilar material qualities between the new finishes and the weathered rock became increasingly contrasting. The introduction of uncoated steel that reacted to the environment created a mediating material, manufactured yet weathered like the rock.

Future implications of an unconsidered design decision became highlighted through the construction of a garden. Without a clear understanding of the prevailing climatic conditions or the level of maintenance that was required, the garden increasingly deviated from the initial unsustainable design vision. The plants that self-seeded were by definition ideally suited to the environment as they had emerged from and were, thus, selfsustaining.

From the construction of a building came an understanding of the importance of site, context and time in the design process. The question that remained was how this understanding could be translated into the creation of products that were sited on the human body. 
5.2 Displaying Wear 
Experiments: \# $2.1-2.40$

Materials: Matt black paint (5 microns) / Clear plastic (Acrylic)

Primary design issue: Research methods to simulate and display wear relative to form. 


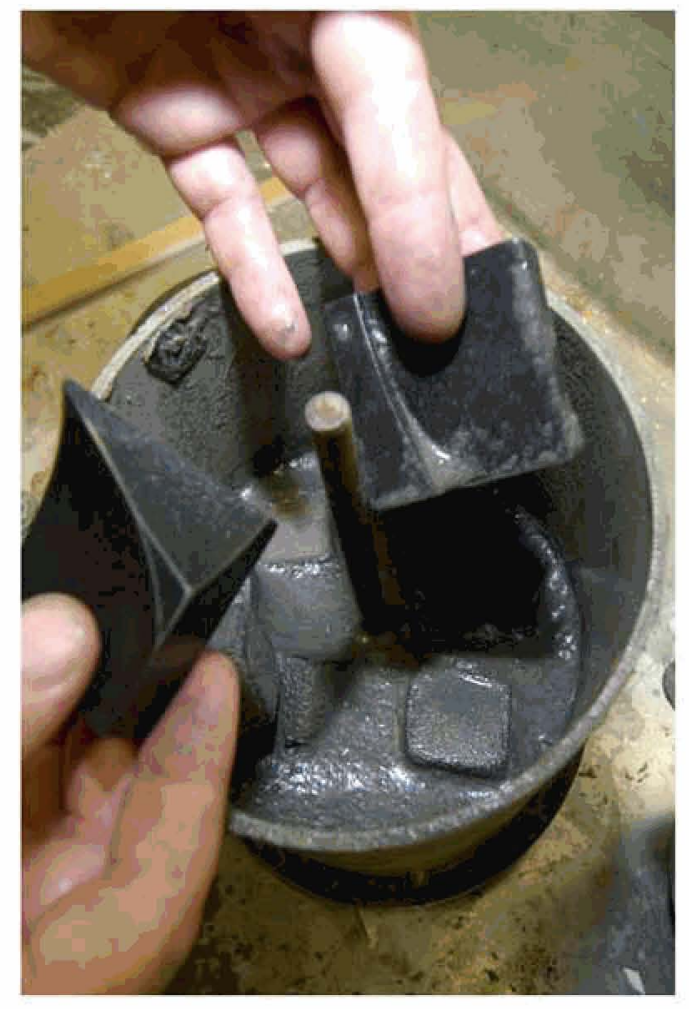

Wear simulation process

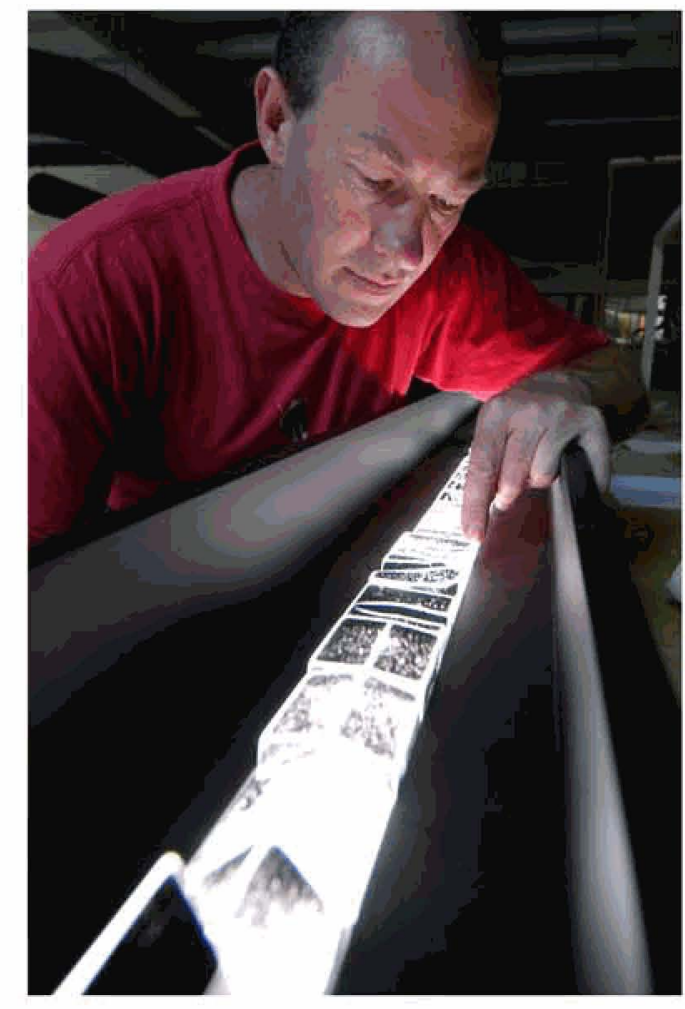

Wear evaluation 


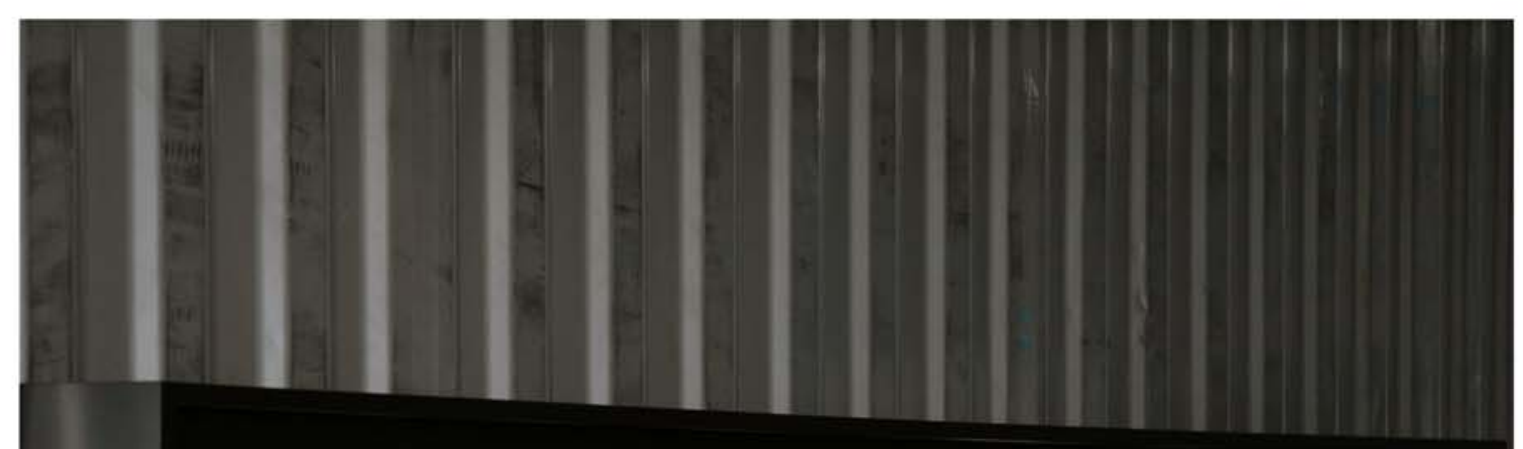

$10.1000 .10000 \cdot \cdot 0$.

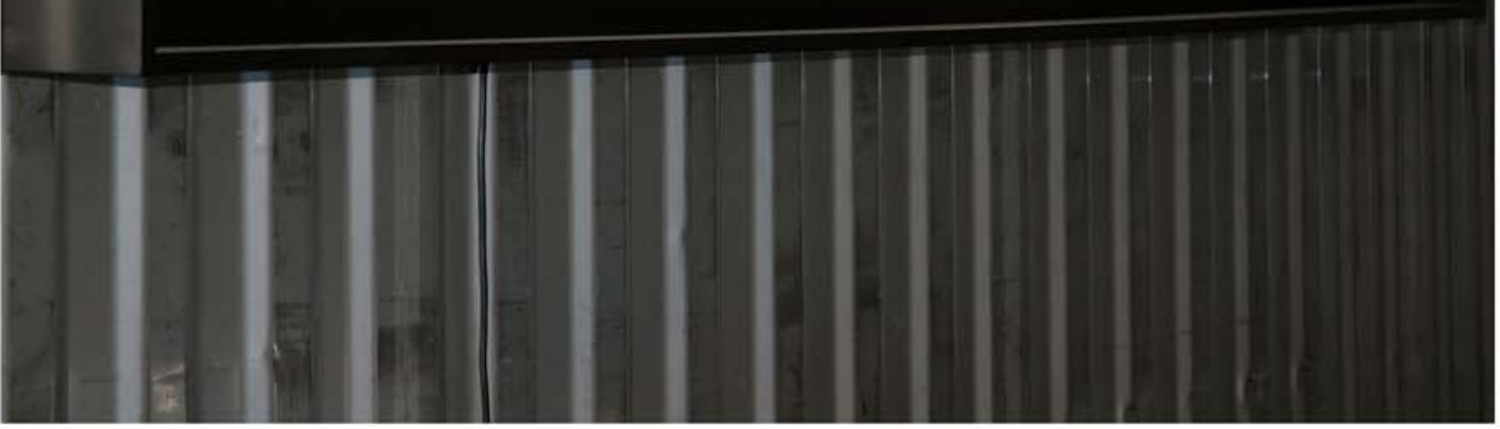

Displaying wear 


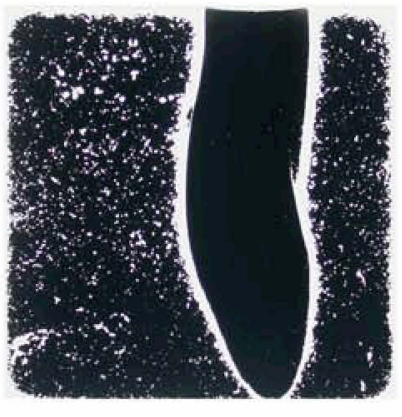

\#2.1

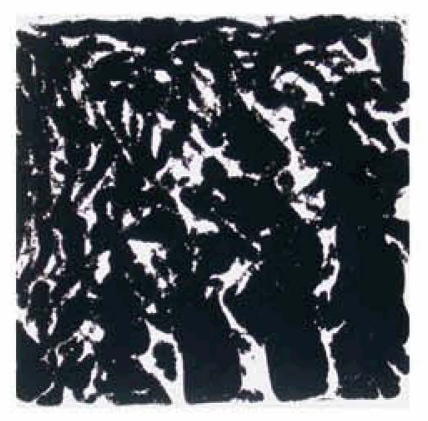

\#2.5

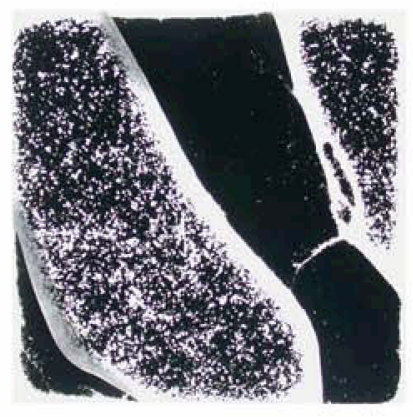

\#2.2

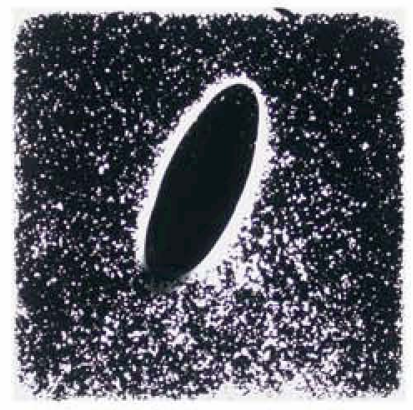

\# 2.6

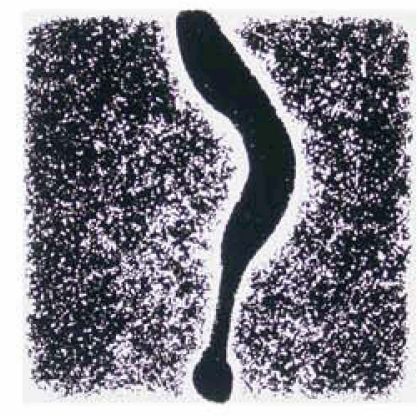

\# 2.3

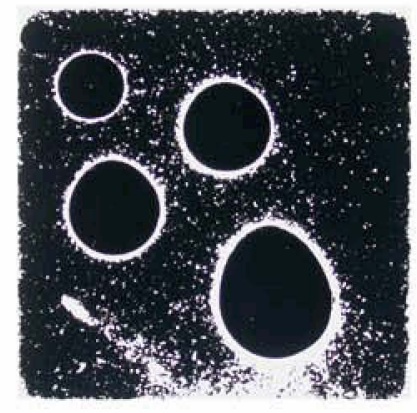

\# 2.7

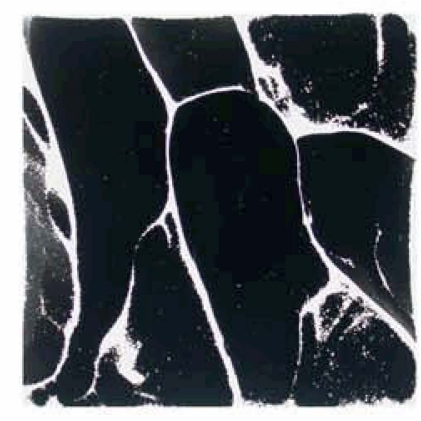

\#2.4

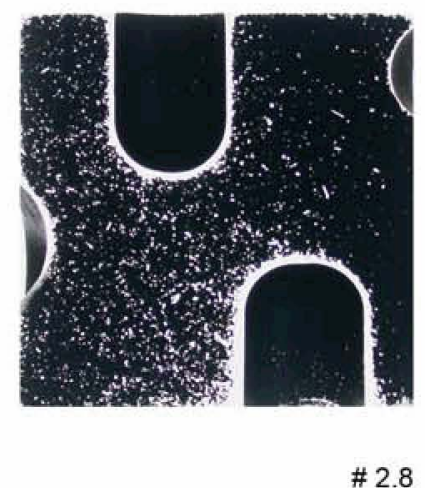



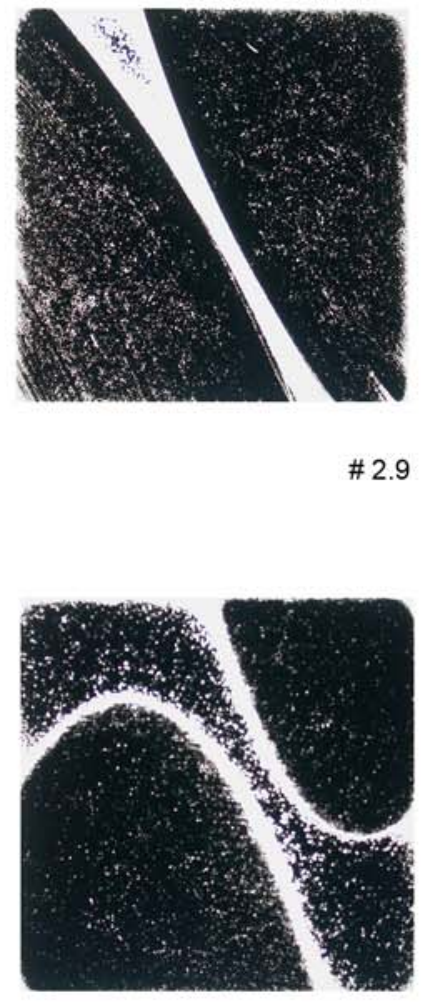

\#2.13
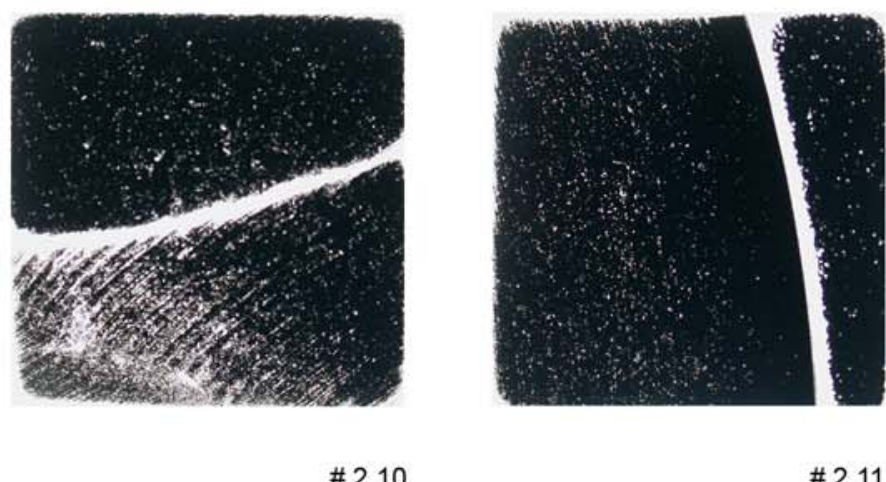

\#2.11

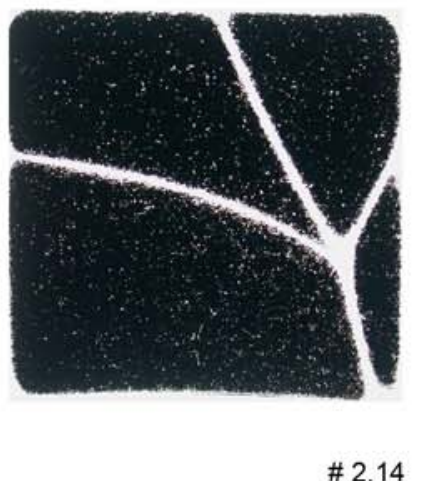

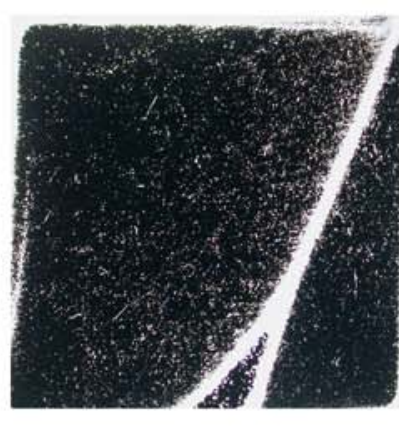

\# 2.15
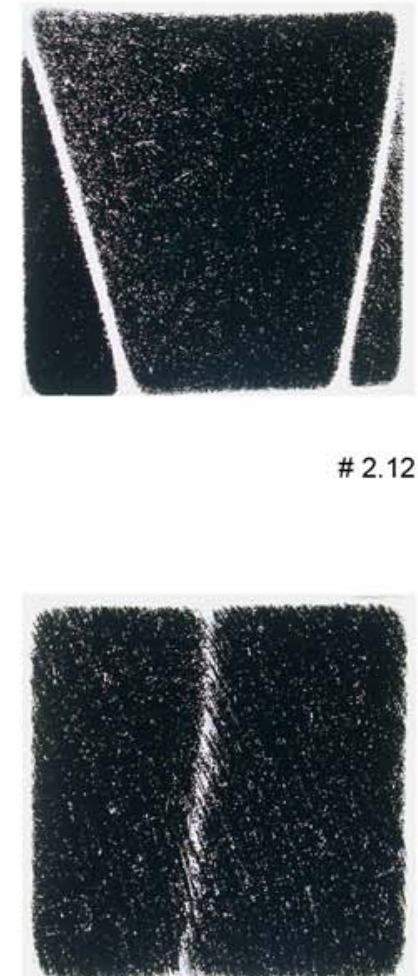

\#2.16 

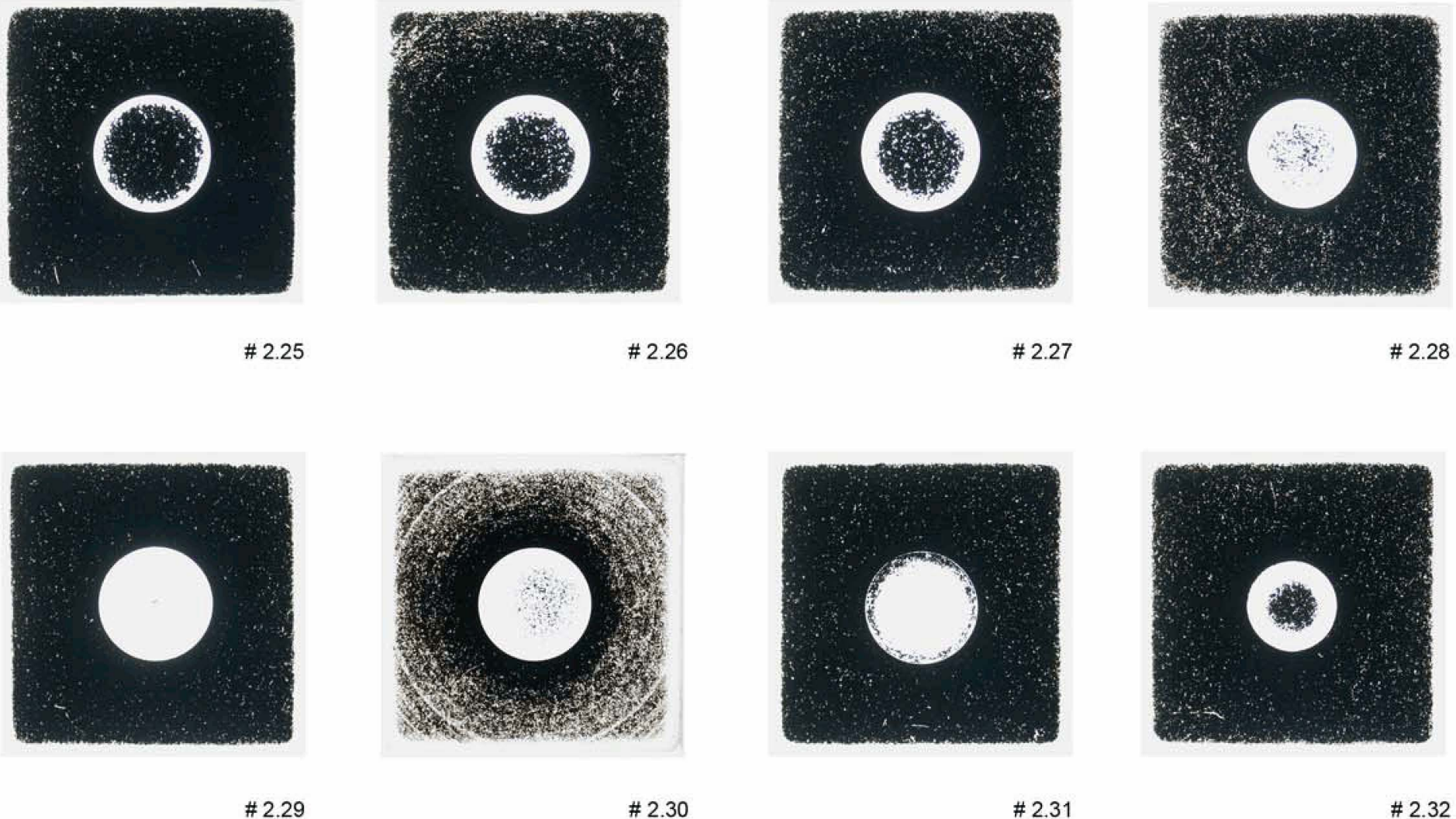

\# 2.30

\#2.31

\# 2.32 


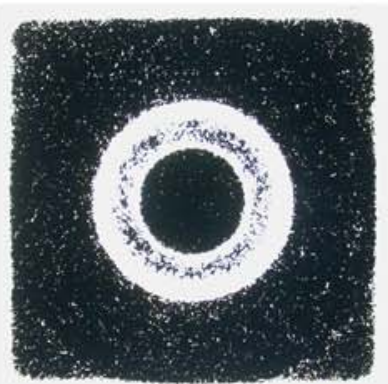

\#2.33

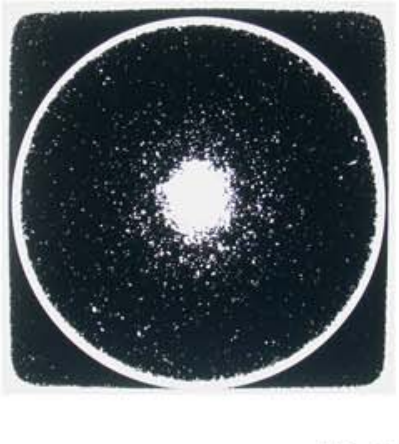

\# 2.37

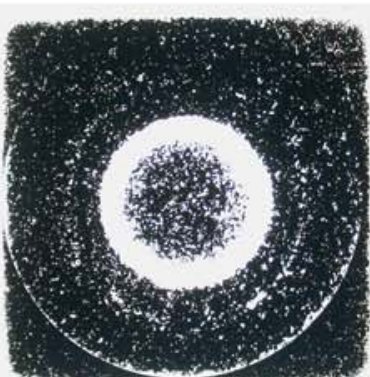

\# 2.34

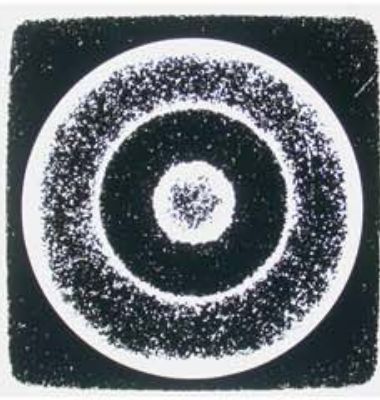

\#2.38

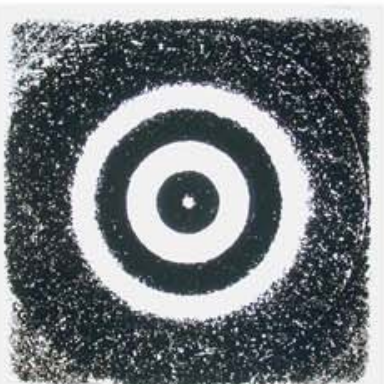

\#2.35

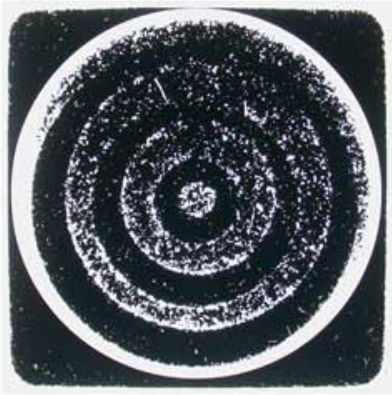

\#2.39

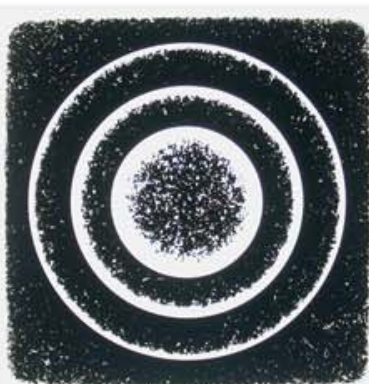

\#2.36

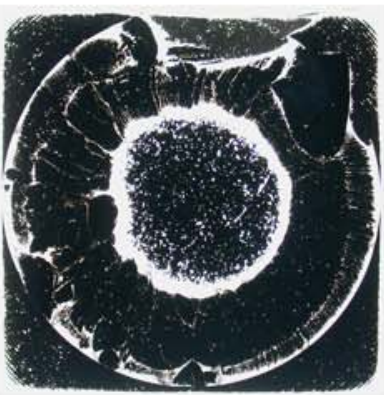

\#2.40 

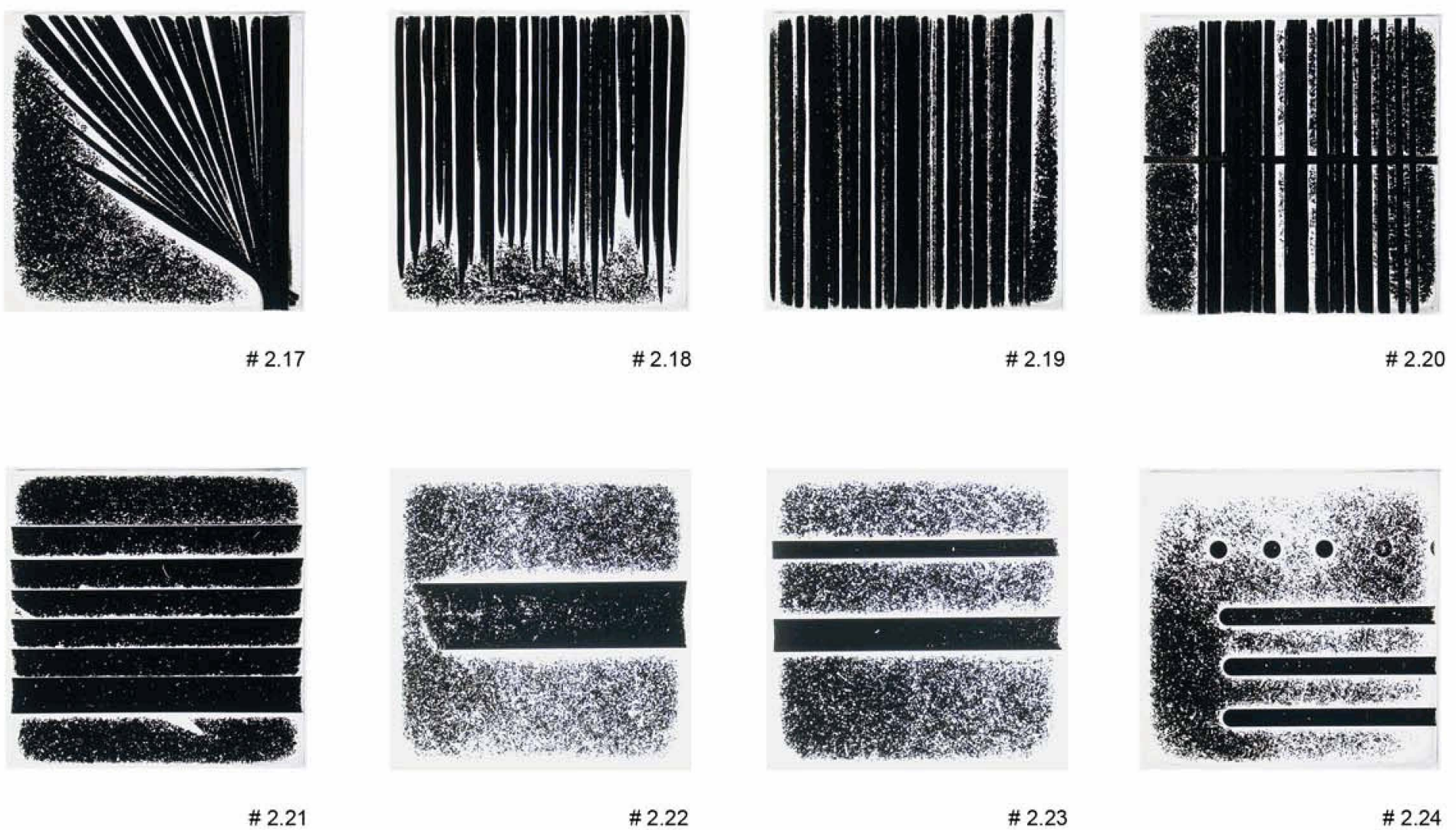


\section{Observations}

The qualities in the worn samples held an association to print making with subtle transition from opaque black through to bright white. A grey developed from the light escaping through the increasingly translucent and fragmented black (Ref sample \# 2.30). Perceived this way, form and wear could be understood as a method of image making, largely predetermined, yet capable of surprises created through the process. Unlike $2 D$ prints these products are $3 \mathrm{D}$ with multiple perspectives from which to be viewed and with tactile surfaces to be touched.

Evidence of the tools and physical actions used to create the samples became increasingly visible as the top surface eroded allowing the light to shine through the ridges of the textures created during construction (Ref sample \# 2.10) The process of "finishing" the samples with a consistent painted skin and then subjecting them to hours of abrasive re-finishing formed an interaction between the product's form and surface coating that created a site specific finish similar to the qualities of weathered buildings.

The expectation that the level of control in the processes used to create the samples would be fundamental to the result initially appeared

unfounded. The samples made using rough hand tools (Ref sample \# 2.4) or precise and prescribe machine tools (Ref sample \# 2.36) appear equally enhanced through the actions of wear.

The issue of product enhancement through wear appeared more related to the designer's intention that products should change as a deliberate and valued act. The use of light to reveal wear inverted the association between wear and decay into one of wear and display.

The capacity of wear to reveal subtle surface detail (Ref samples \# $2.16 \& 2.24$ ) suggested that products could be produced using very precise details that would only become visible once the product was used. With this process products could reveal new details as they aged.

$$
\text { (ref 5.4 Multiple Messages) }
$$

Defining the 'end' moment, when to finish working these samples, became problematic. If the process continued endlessly then it could reveal further interesting reactions, but if worn too far the existing evidence could be lost. The inherent variability of the process meant that the product could not be created identically. To allow the wear process to extended through multiple 'end' points required the production of multiple samples or the recording of the wear process 


\subsection{Wear Stages}


Experiments: \# $3-3.15$

Materials: Coloured paint (5 microns) / Clear plastic (Acrylic)

Primary design issue: The potential of surface detail to be embedded into a product and revealed through wear 


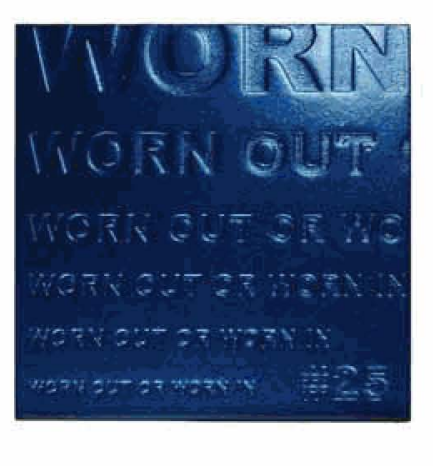

\#3

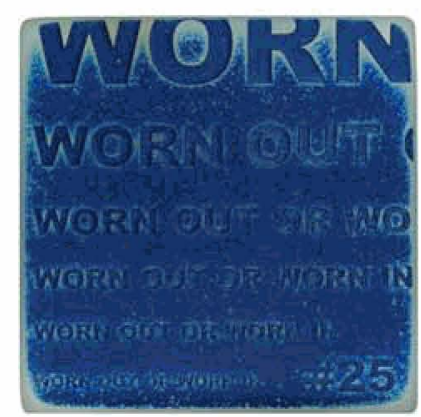

\#3.1

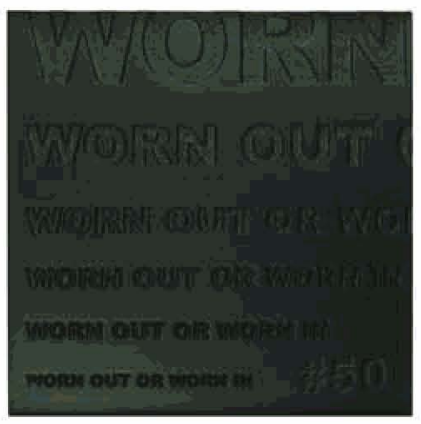

\# 3.4

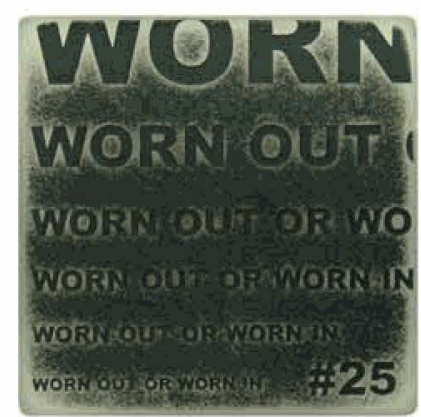

\# 3.5

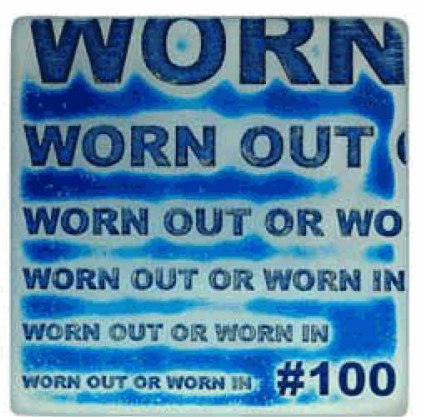

\#3.2

WURN WORN OUT WORN OUT OR WO WORN OUT OR WORN IA

WORN OUT OR WORN IN

NORN OUT OR WORN IN \#10

\#3.3

\section{WORN WORN OUT WORN OUT OR WO WORN OUT OR WORN IN WORN OUT OR WORN IN FORN OUT OR WORMIN \#100}

\section{CUTK WORN OUT WORM $2 L-=F W$. WORN OUT OR WORN WORN OUT OR WORN IN VORNOUT OR WORNIN \#10}

\# 3.6 


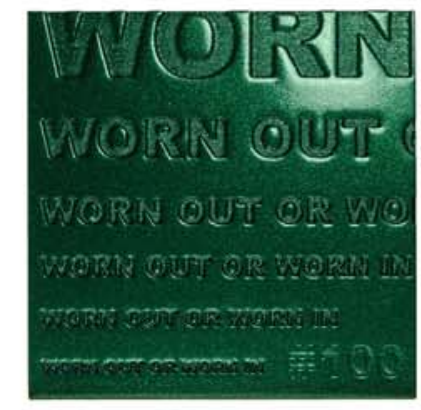

\# 3.8

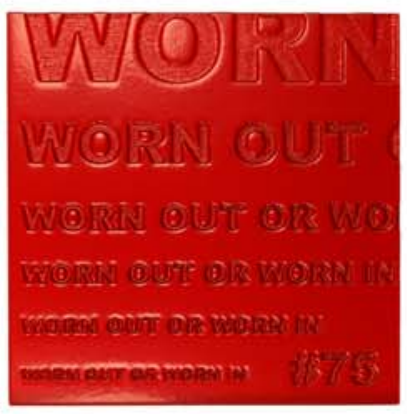

\#3.12

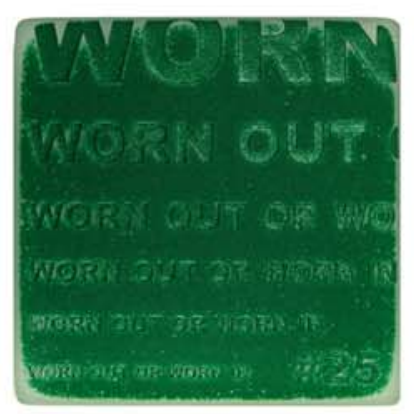

\# 3.9

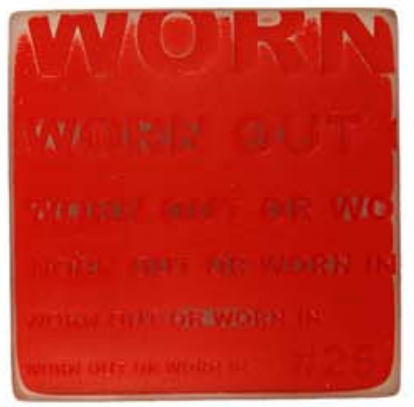

\# 3.13

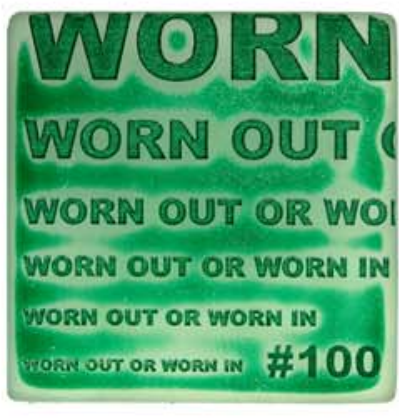

\# 3.10

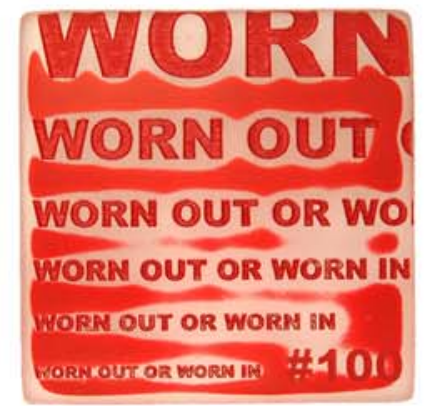

\# 3.14

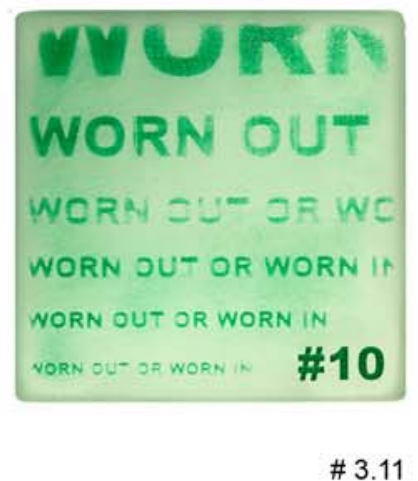

VVUR WORN OUT WORR ZU- DR WC WORN OU- OR WORN IN WORN OUT OR WORN IN \#10 


\section{Observations}

The potential revealed in the earlier experiments for subtle details in the products surface to be exposed through the wear required a more precise process to be explored. The laser engraving process was selected because it offered flexibility, consistency and precision (1200 DPI). As a tool it occupies an area between 2D printers and 3D machines which are capable of creating complex forms. Programming it required the use of a basic drawing program (Macromedia Freehand).

By modifying the power and speed of the laser, variations in the depth of the engraving can be created. A series of font sizes and depths were used to explore the limitations of the text to remain legible as the surface wore. The text engraved at the finest depth ( $<3$ microns) was visually imperceptible once the sample was painted. As the paint layer was removed the text became visible yet was still imperceptible to the touch.

The use of a lapidary tumbler to synthetically wear the samples added consistency to the process that allowed for a complex interaction between the product's shape and the wear mechanism. This result revealed the potential diversity and visual qualities of wear but did not represent a likely wear mechanism that would affect hand held portable products. For that reason more representational wear simulations needed to be developed.

The use of a precise process to create the initial samples required an equally subtle wear mechanism to reveal the details held within their surfaces.

A series of coloured samples were trialled to explore the importance of colour in the perception of wear. An unexpected variation in the paint durability occurred: the blue pigments being easily worn requiring 16 hours in the lapidary tumbler to become heavily worn (ref sample \# 3.3 ) and

the green pigments being very wear resistant requiring 42 hours in the lapidary tumbler to create the equivalent wear intensity (ref sample \#

3.11). This offered an insight into the potential subtleties of surface finishes that could be harnessed to create wear, dependent on a durability of specific paint colours and associated pigments.

The fluid state of the paint when it was applied allowed surface tension to create variations in the film thickness. This became especially apparent around the text that had been engraved at greater depth into the surface (ref sample \# 3.14). 
5.4 Finger Wear 
Experiments: \# $10-10.4$

Materials: Red paint (5 microns) / Plastic (Acrylic)

Primary design issue: The size of wear patterns generated by the finger 


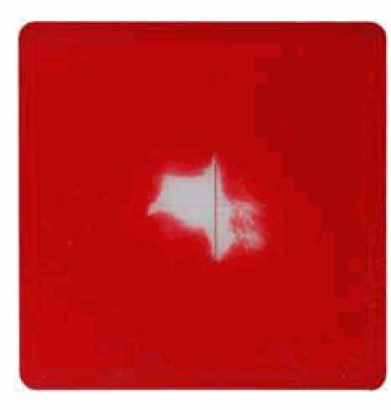

\# 10

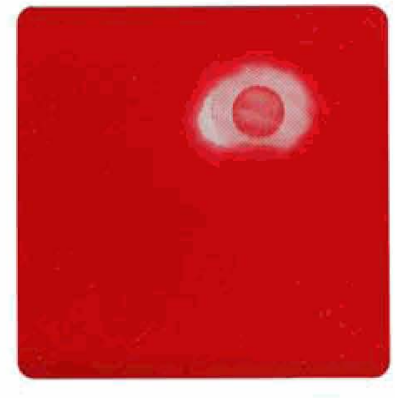

\# 10.1

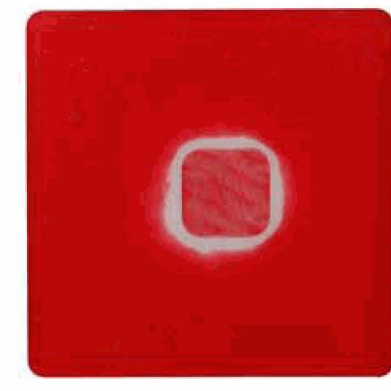

\# 10.2

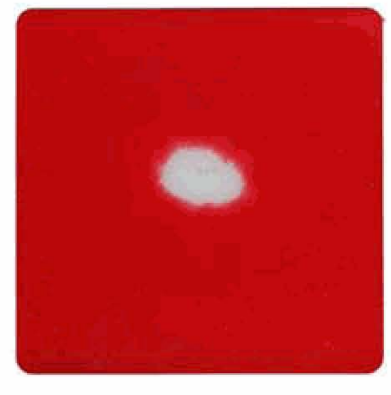

\# 10.3

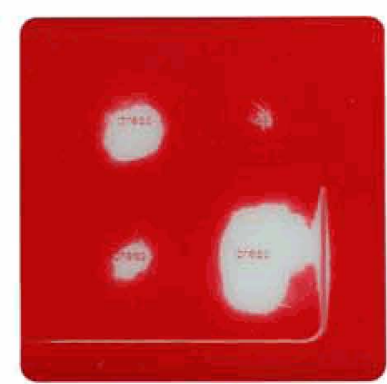

\# 10.4 
Observations

The 1:1 scale images on the opposite page illustrate the size of the wear pattern created by a finger repeatedly pressing down on a flat surface. As the wear process continues the area becomes larger until it reaches the limits of the fingers size and the range of its movement (ref sample \# 10.4)

As a wear mechanism the finger is much finer than the previous tumbling process, the friction between the skin and the product is minute resulting in a slow polishing process which is capable of revealing the most subtle of graphics details. The 1200 DPI resolution on the laser engraver used to create the samples became the limiting factor in establishing small legible text, not the wear mechanism of the finger (ref sample \# 10.3).

The heat generated during the laser cutting process melts the surface of the plastic creating a raised edge around the cut. This minute protrusion focuses the wear energy unevenly on the surface creating an area of increase wear around the line. This initially invisible deformation is made visible revealing the capacity of wear to highlight previously hidden details (ref samples \# 10 \& 10.4). 
5.5 Interface Wear (controls) 
Experiments: \# $10.6-10.8$

Materials: Red and White paint (Acrylic lacquer) / Plastic (Acrylic)

Primary design issue: The role of interface design in dictating the actions of people and resulting wear patterns. 

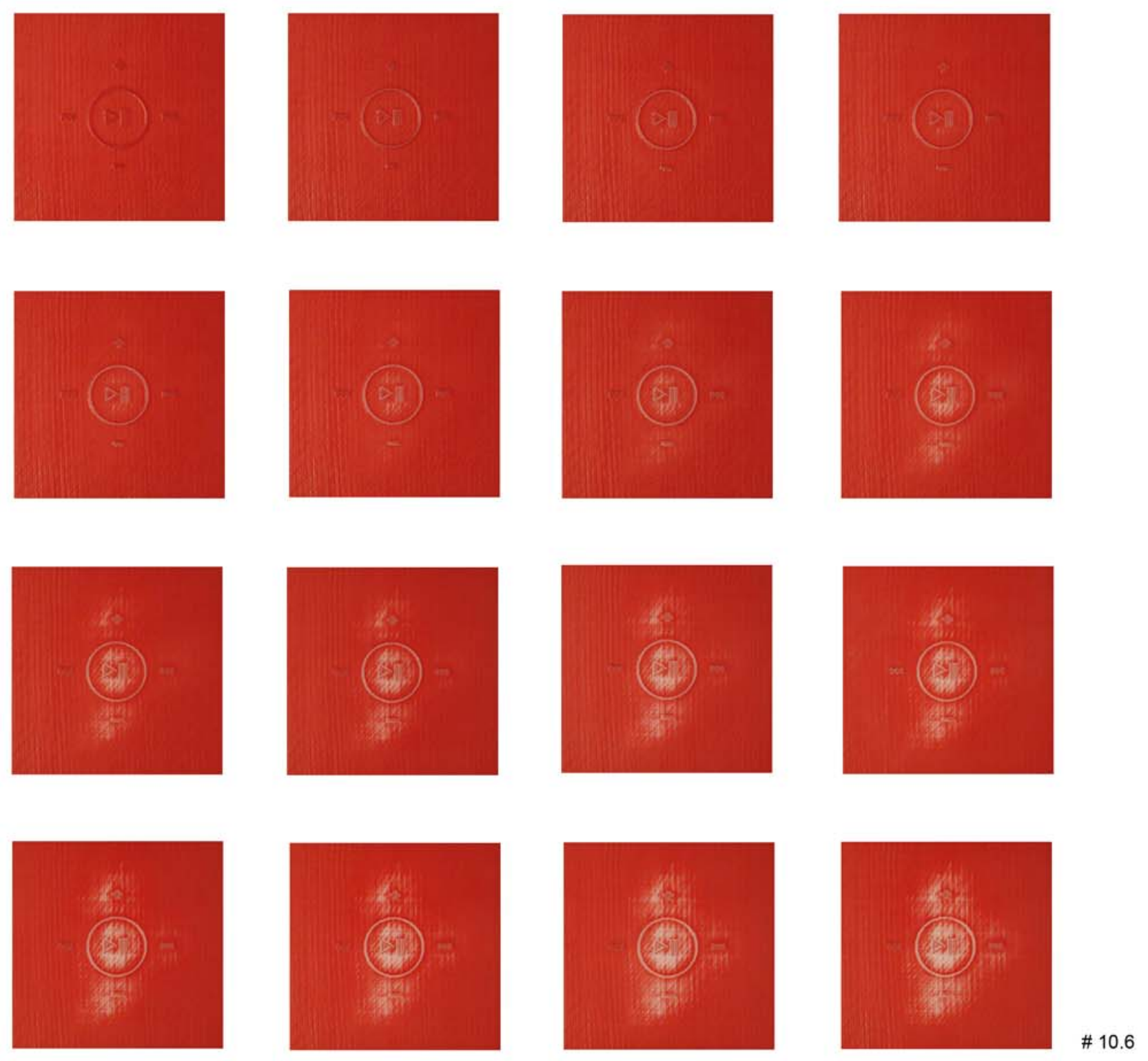

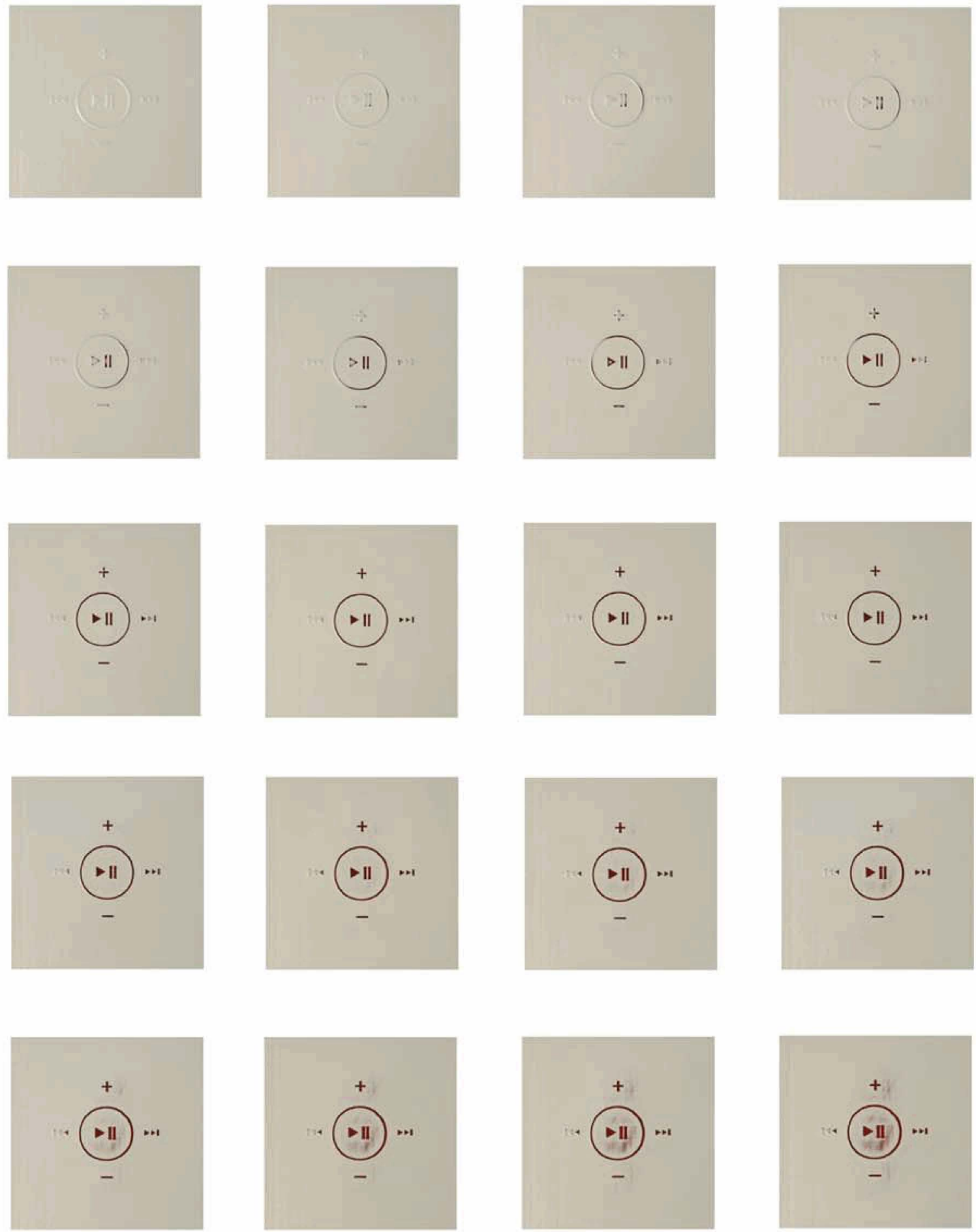

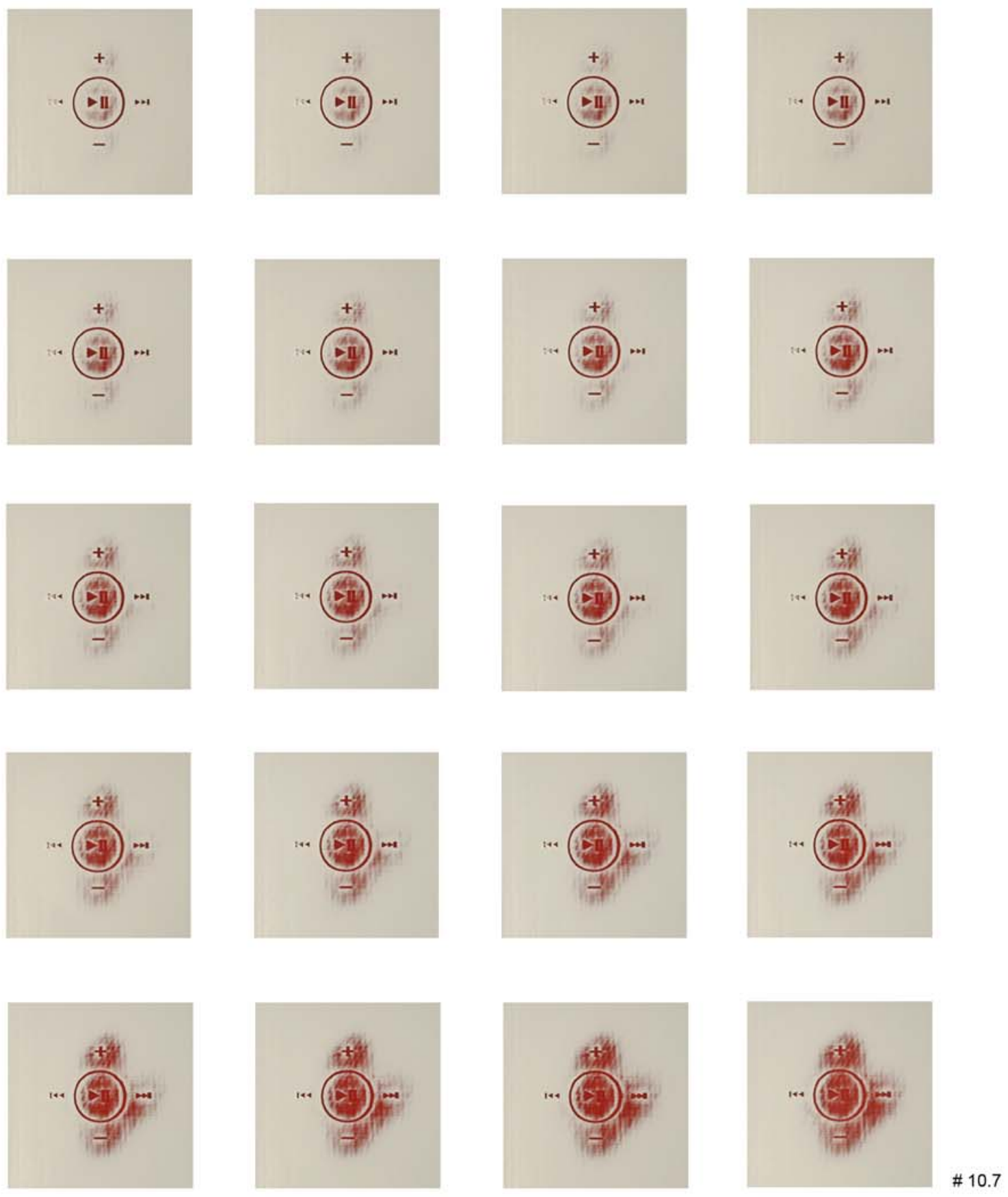

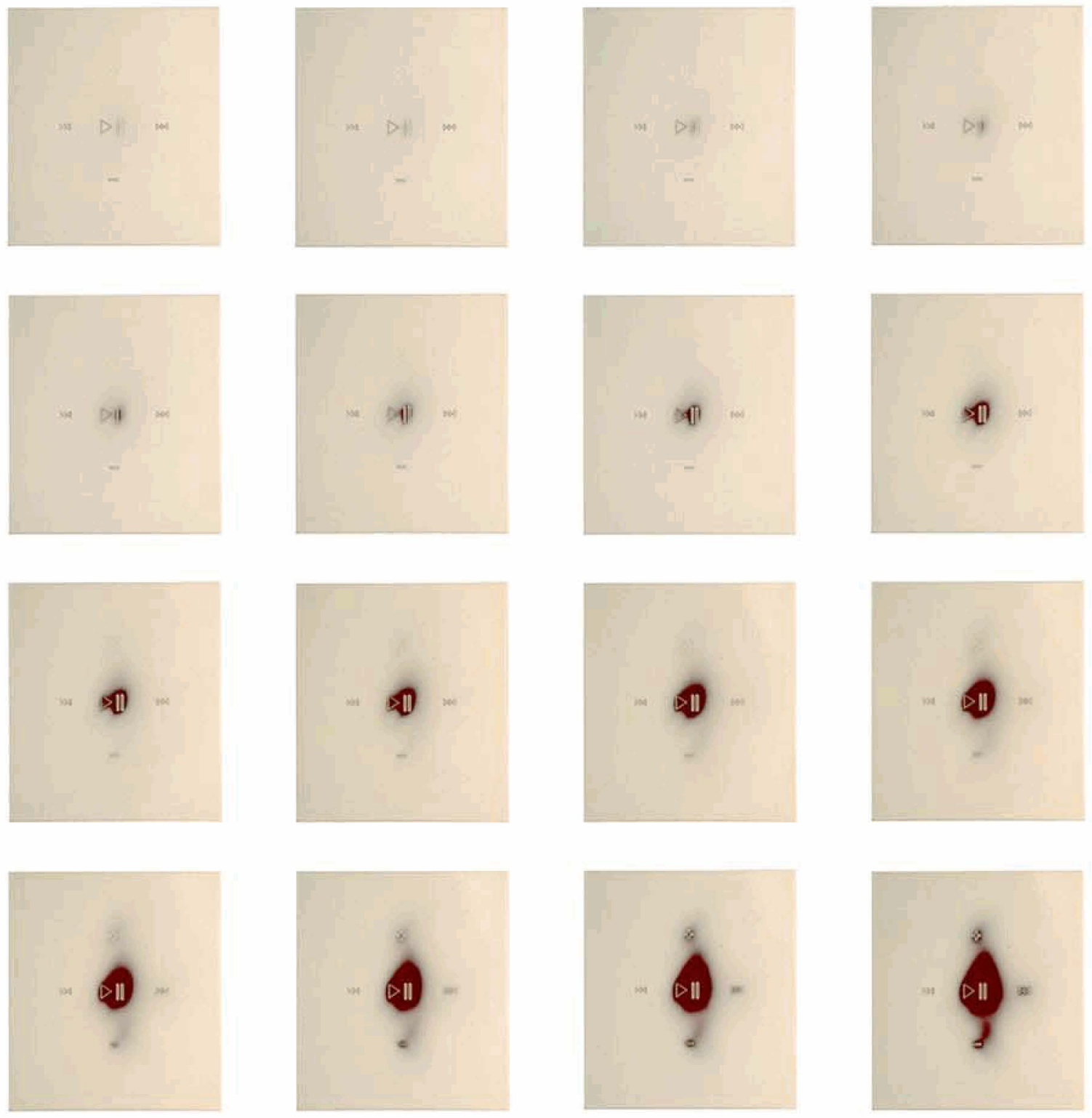

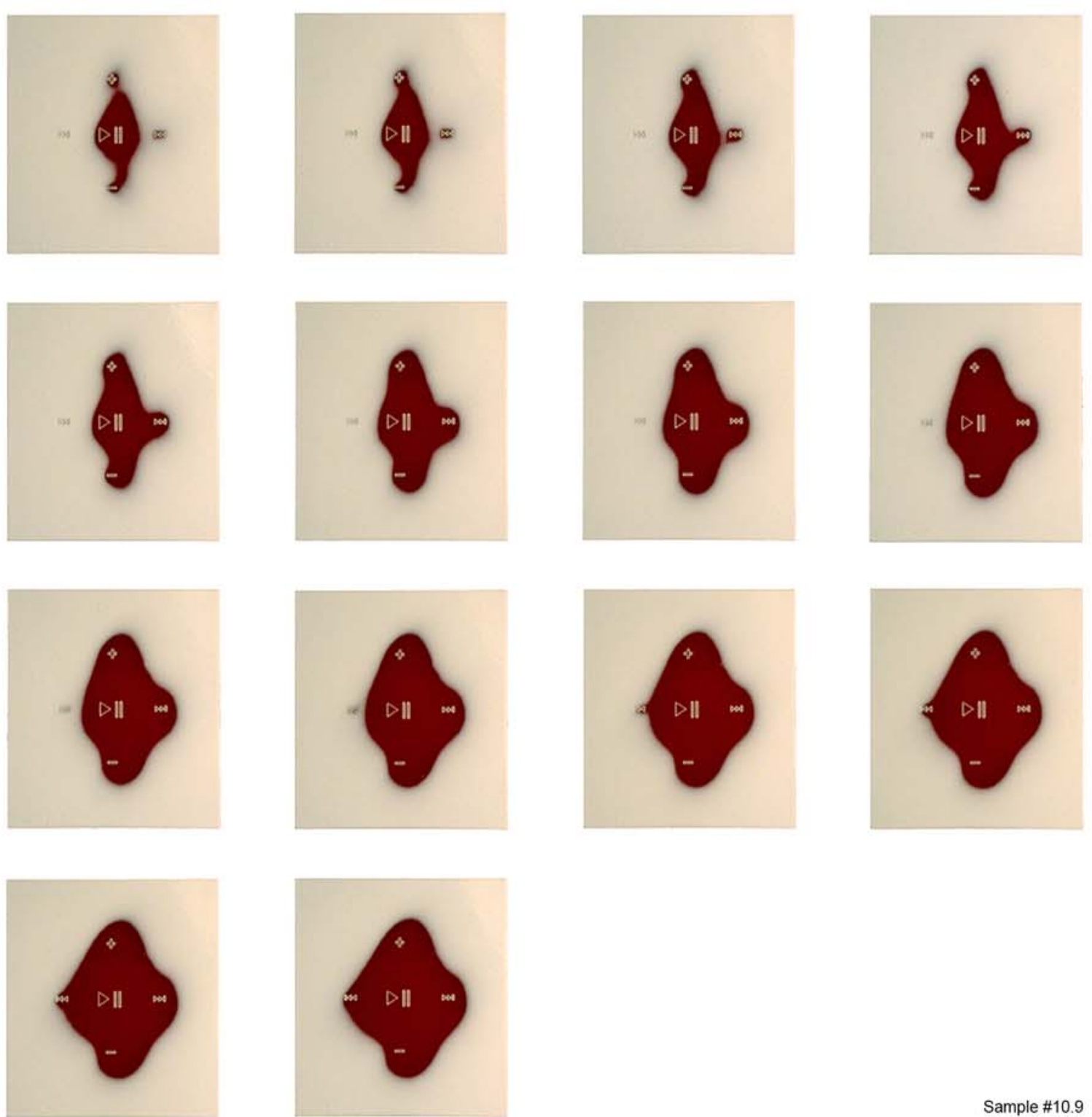

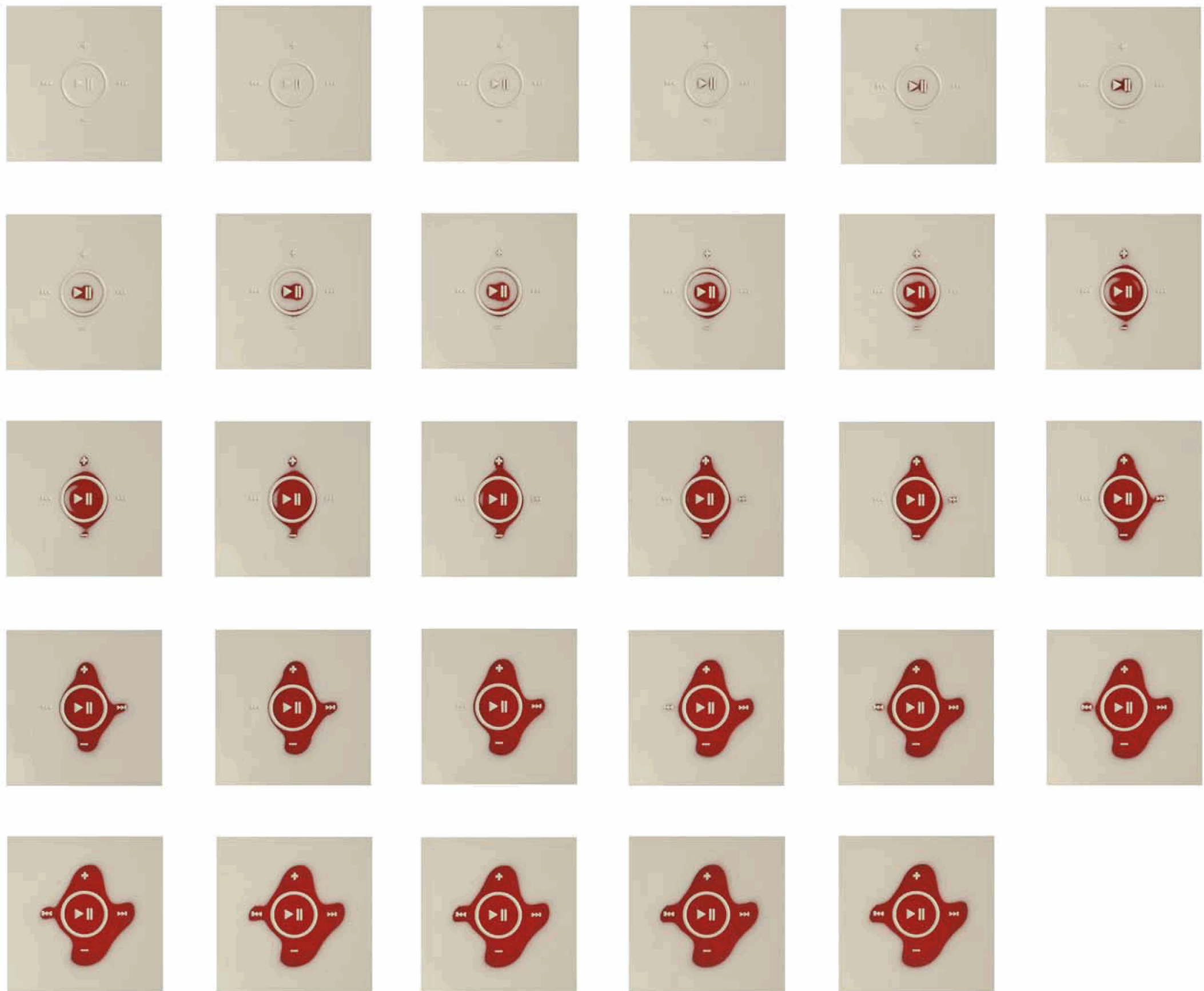
Observations

The edge between the paint and the plastic became softened as it wore, creating a translucency in the initially opaque paint. This revealed the pathways of the finger as it moved between the distinct controls (ref sample \# 10.9). These pathways became more defined eventually linking the distinct controls into one continuous, yet, use and software distorted pattern.

Protruding controls focused wear onto a smaller area that in turn created a faster wear rate. This process transformed the initial subtle controls into fleetingly vivid and clear graphics that degraded into increasingly ill-defined and illegible patterns (ref sample \# 10.7). Recessed controls were initially difficult to read yet through wear became increasingly visible. The heightened need for clear graphics when the product was new and unfamiliar required the reverse process so further research was needed.

The play/pause graphic was the first to emerge through the paint layer as it was used each time when the product was used. The volume up and down controls followed in unison. The use of the forward track button seemed more appealing than searching backward to music previously listened too. This simulation was based on a personal perspective of listening to music. Of interest is not how accurate the

simulation technique is but rather how limited it is in representing the array of people's physiology and personal preferences. The emerging goal of a $4 \mathrm{D}$ approach to product design is diversity created through the construction process of use.

The animations created by sequencing together the still images (ref enclosed CD) allowed a fluid and compressed understanding of the wear process. The animations could be understood as historic records or future predictions depending on the viewer's perspective.

The most important quality in these diverse wear patterns was the evidence of human occupation. The patterns echo conscious and considered actions that had been performed repeatedly over time to accumulate as evidence of the person's connection with the product and their existence. 
5.6 Multiple Layers 
Experiments: \# 11 - 11.3

Materials: White and Yellow paint (5 microns) / Plastic (Acrylic)

Primary design issue: The potential of multiple layers to allow unexpected transformations through wear. 

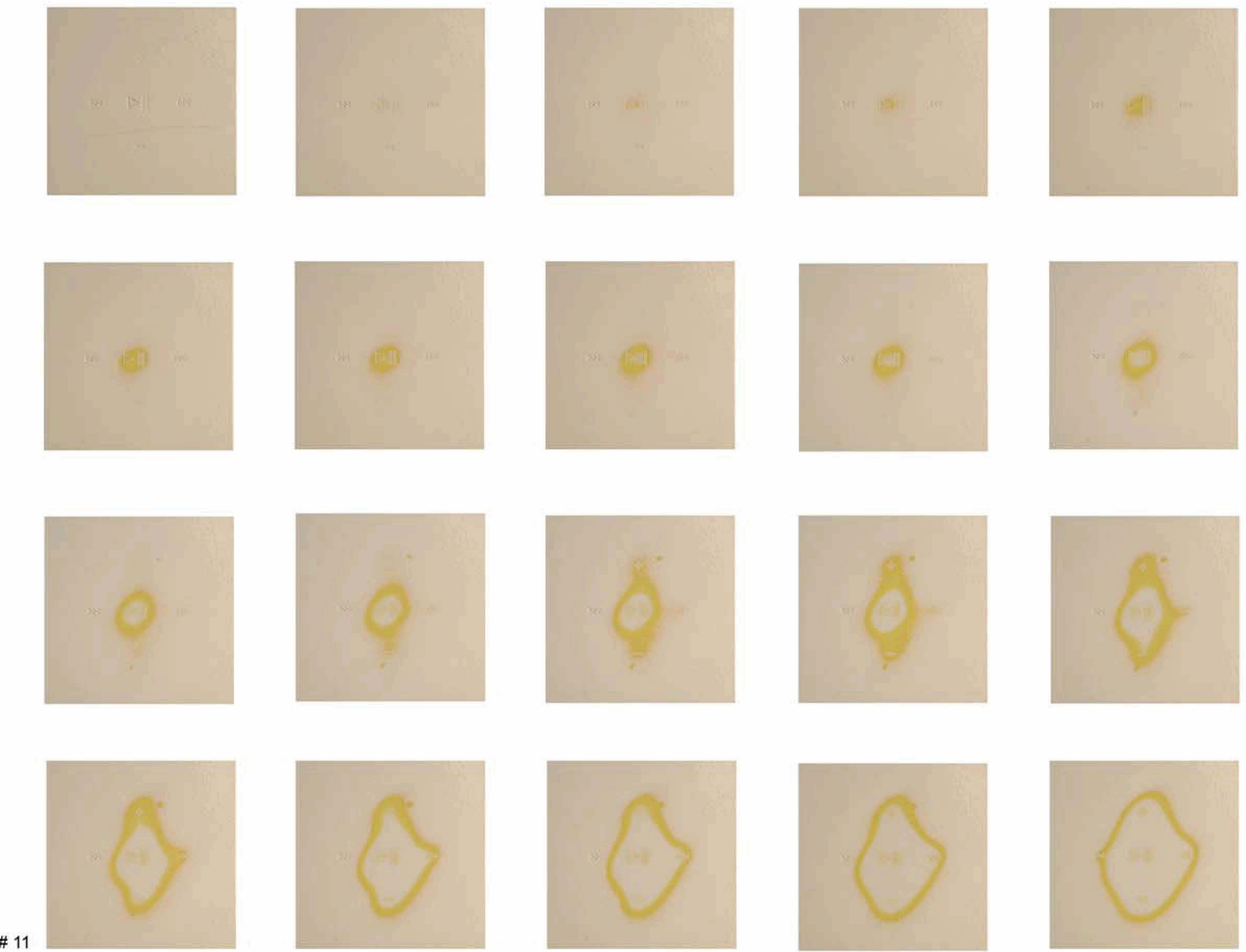

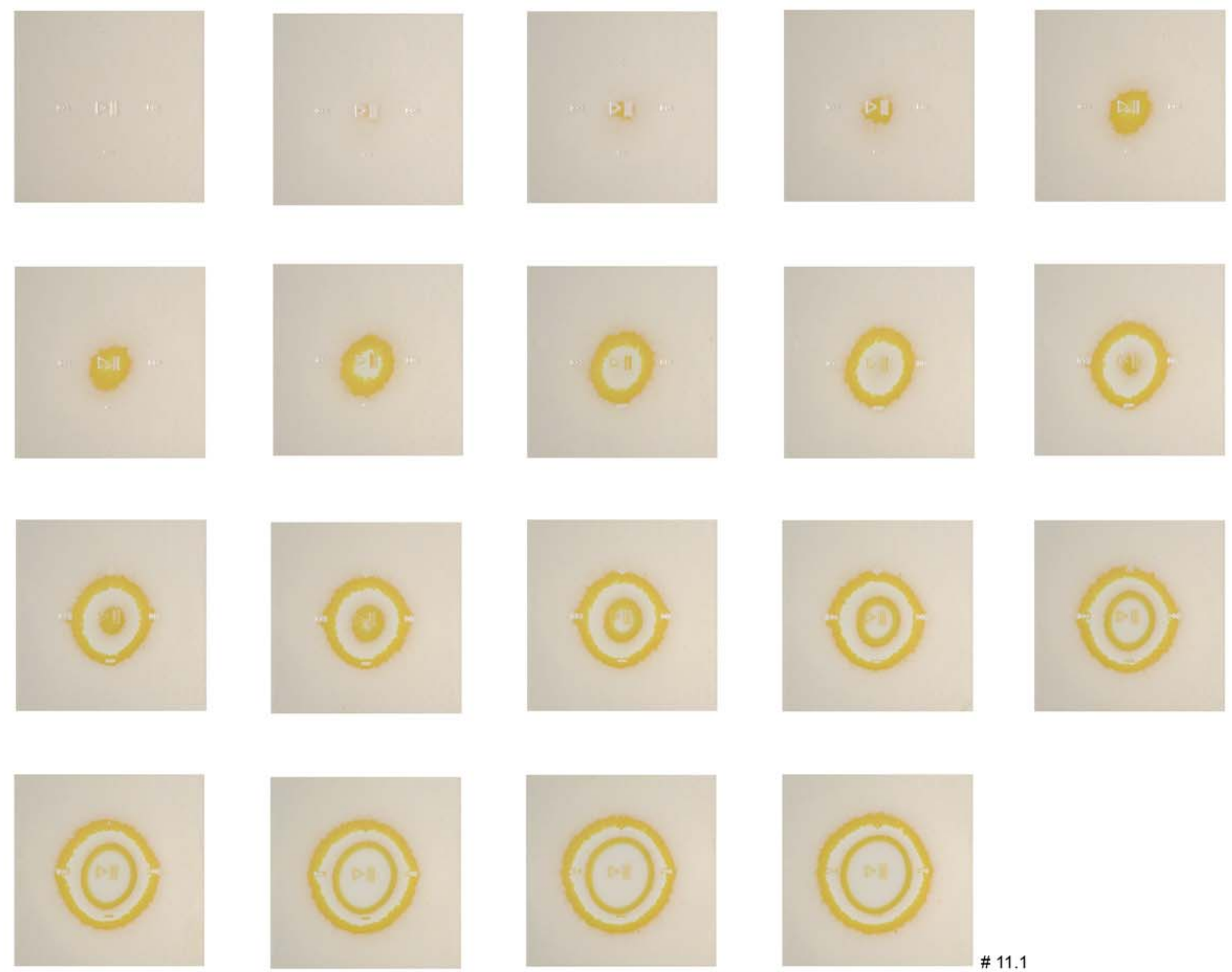

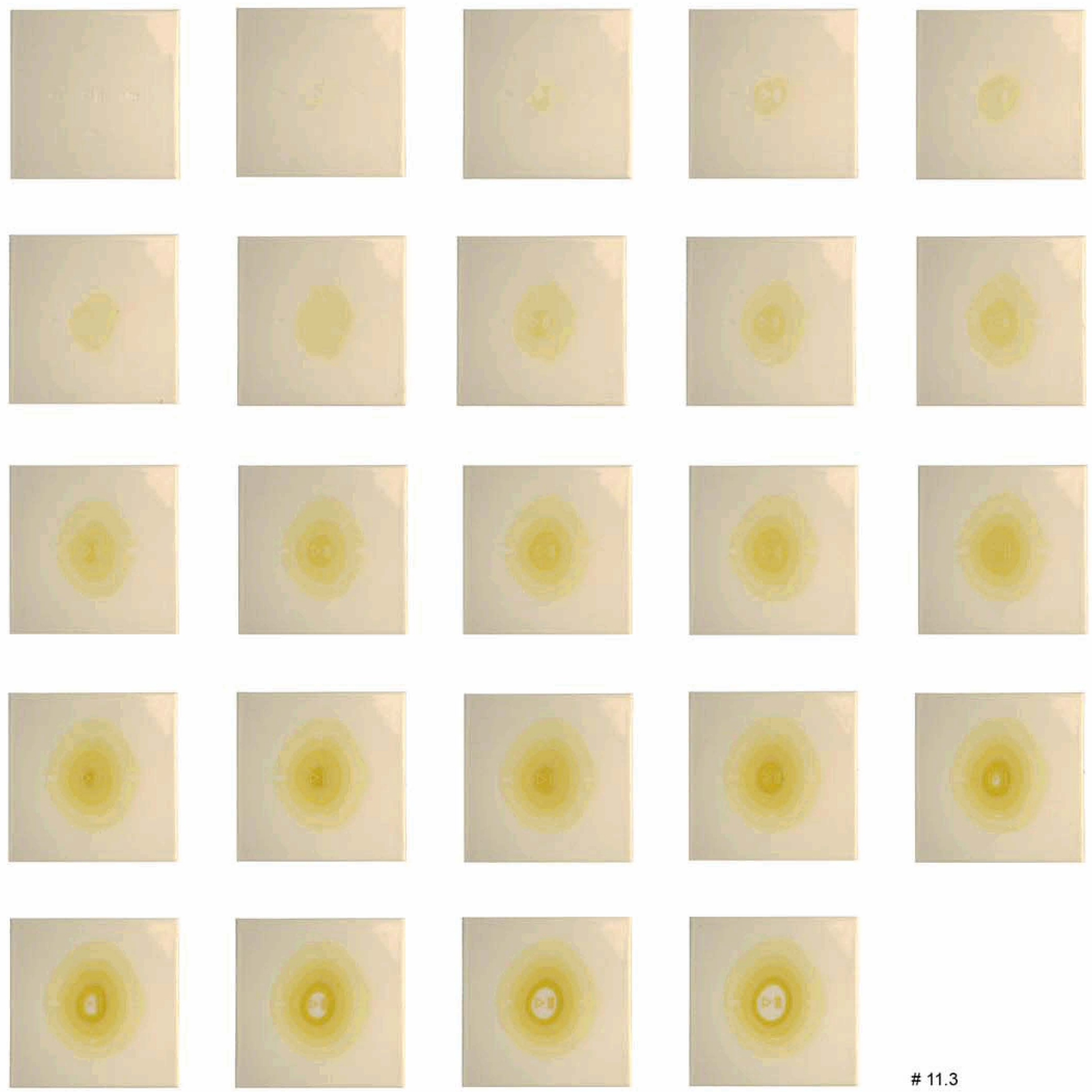


\section{Observations}

The initial desire to create products that resisted wear or transitioned from one surface to another extended into the design of surfaces that underwent multiple transformations. This required another understanding of a product's lifecycle to offer an intrigue and an excitement with hidden potential to become apparent through use. As the product appears to have finished the transition, another change emerges (ref sample \#11.1).

Multiple layers created out of shades of the same colour (yellow) moderates the transition between the exterior and interior of the surface. The contrast between the white base material (ref sample \# 11.3) and the darker yellow creates the necessary distinction to allow the graphic to become legible once revealed. The contrast between the darker yellow and the exterior white is then reduced through sequentially blended shades between the colours. 
5.7 Multiple Messages 
Experiments: \# 12 - 12.1

Materials: Masked Paint Layers (5 microns each) / Plastic (Acrylic)

Primary design issue: The potential to embed multiple messages into a surface that are revealed through use 

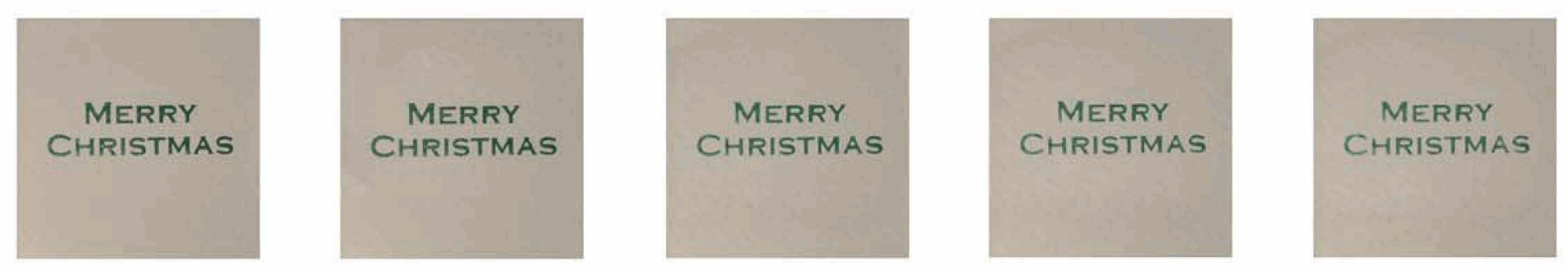

MERRY

CHRISTMAS
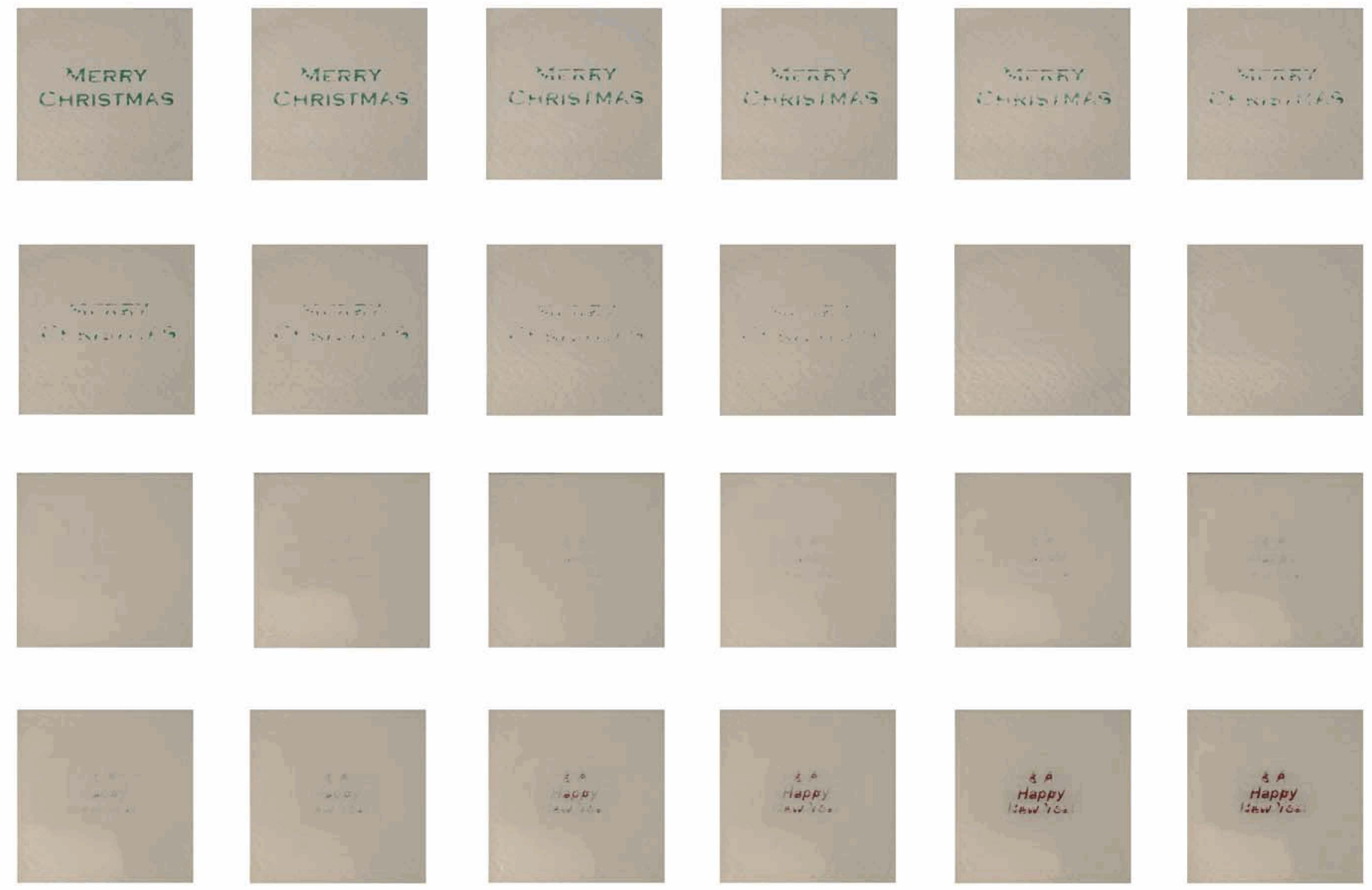

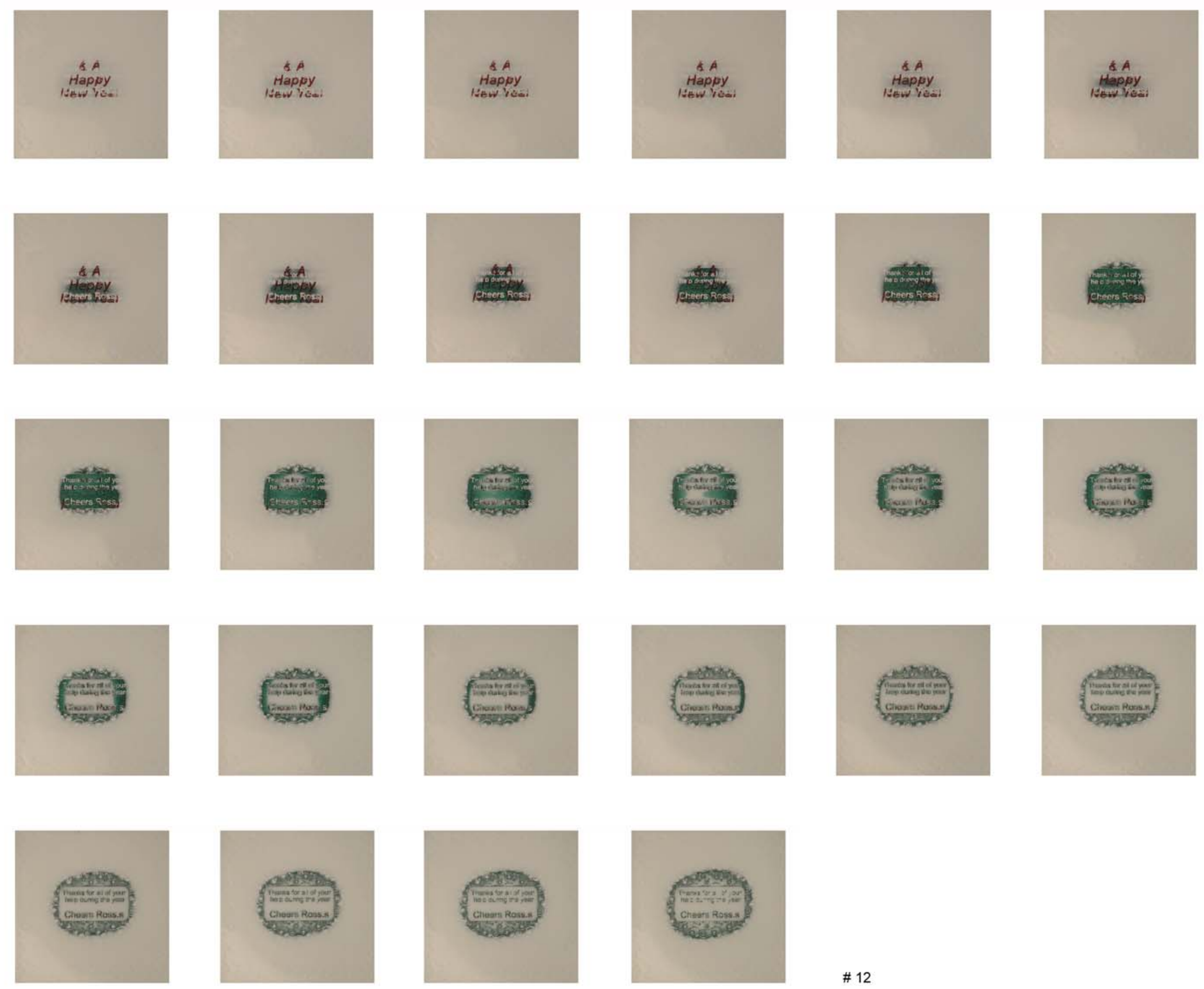

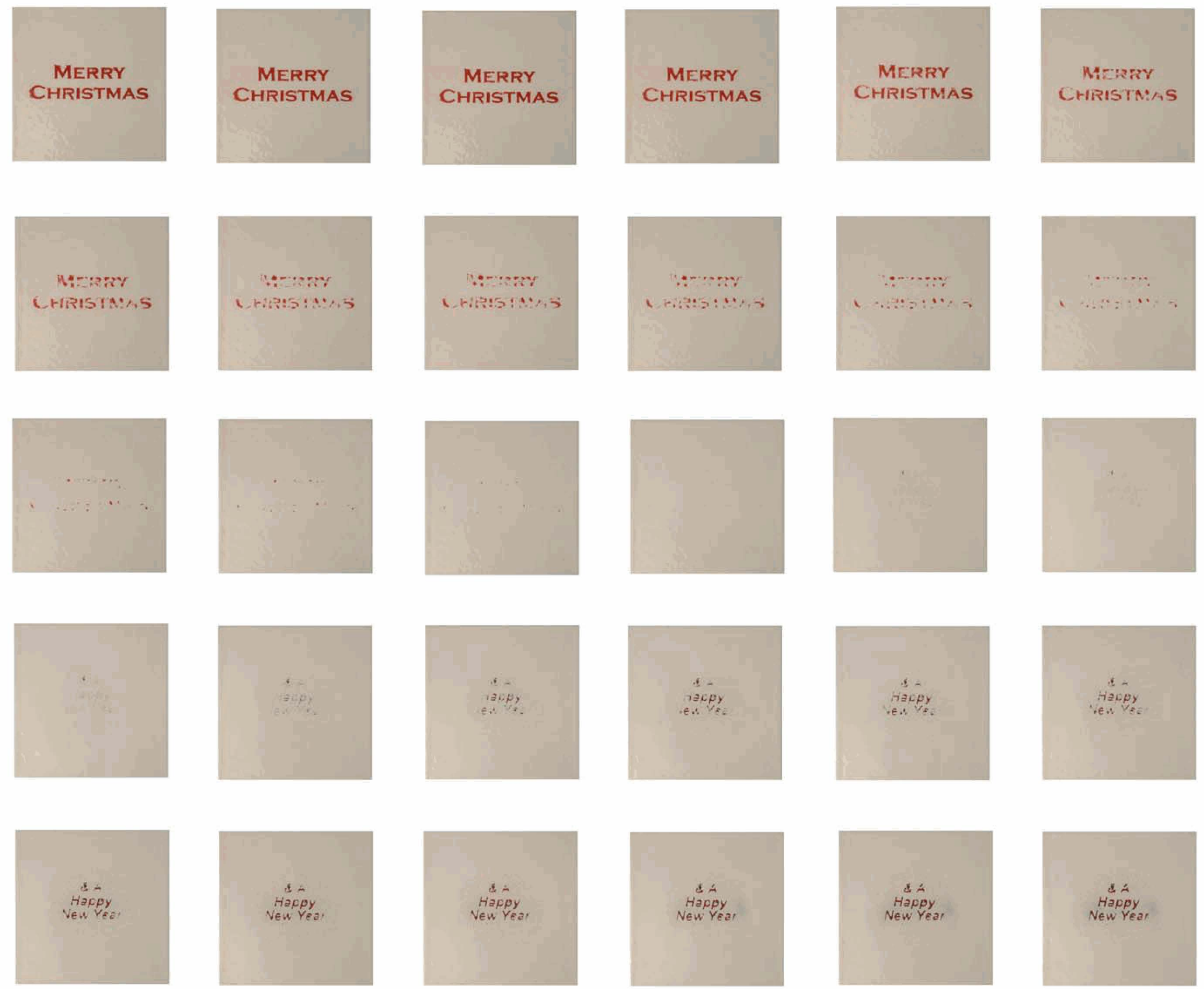

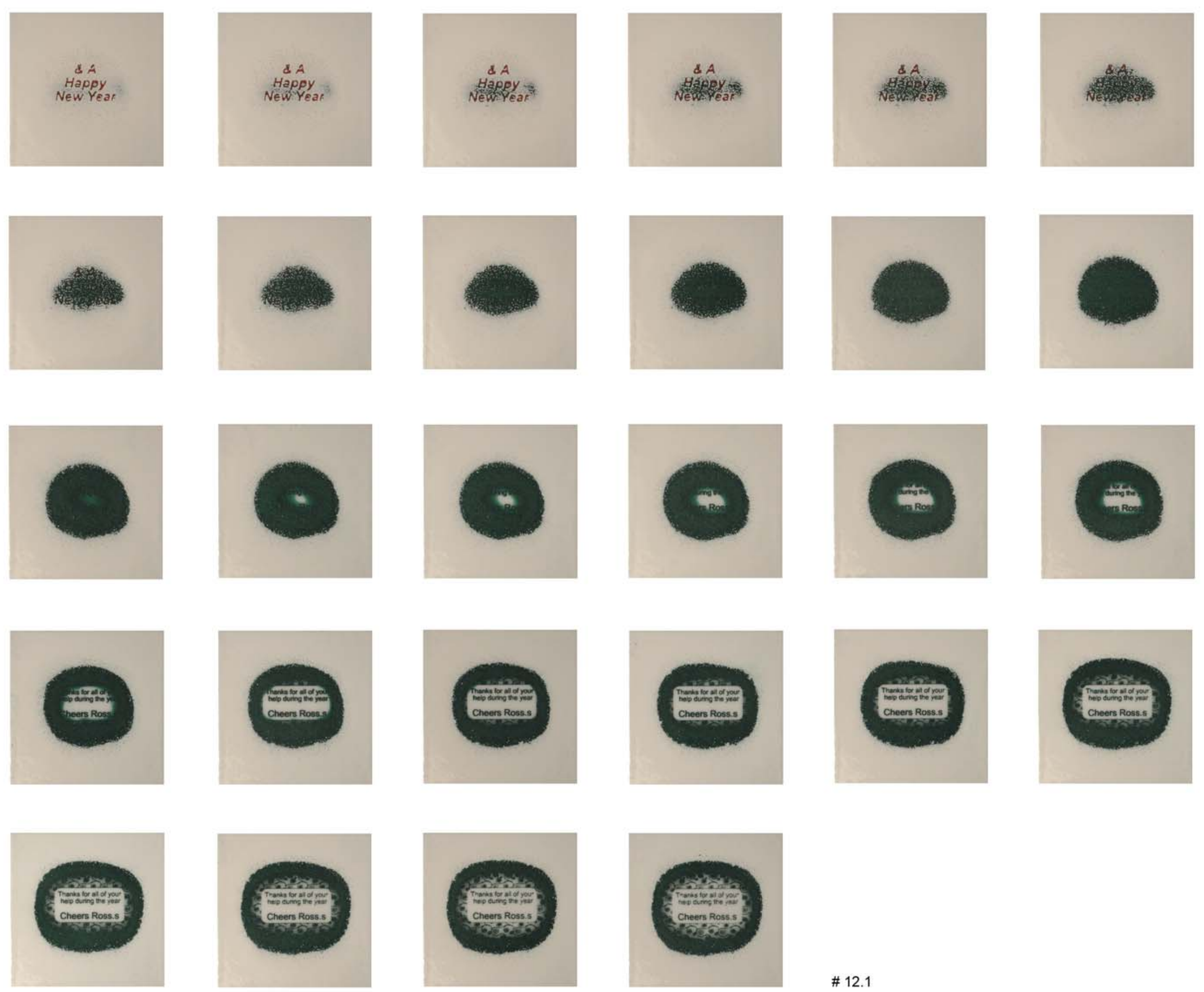


\section{Observations}

Graphic details on a product that transform when used offer a mechanism to reduce information that becomes redundant once the user is familiar with the functions of the product. Conversely if the user is developing negative long-term habits such as R.S.I. that could affect their physiology, the product could reveal messages promoting a change of physical interaction or frequency of use.

The precision of the text on these samples was limited by the mask technique used and for that reason was more variable than desired. It is envisaged that the layer and text would be applied using very quickly cured inks (UV sensitive) and using standard mass production technique

(silk-screens). This would yield a fast, cost effective and consistent application. The base text was laser engraved into the plastic surface. The programmable engraver offers a new potential to manufacture mass customised products with limited additional cost. These embedded messages would be initially hidden and only revealed through use.

The goal of the layers is to get a single clear message to appear at one time. In the first experiment (ref sample \# 12) the layers corrupted each other resulting in the messages becoming mixed and difficult to read. The occurrence of precise messages during the wear process reinforces that it is a deliberate, expected and designed act.

While the focus of this research is on the aesthetic qualities of products, it is critical that the concept and techniques can be transposed into the mass market if the finding are to be applicable to the Industrial Design profession. 
5.8 Interface Wear 3D 
Experiments: \# 4 - 4.3

Materials: White paint (5 microns) / Neon green plastic (Acrylic)

Primary design issue: The application of information gained from pure design experiments into tangible products. 

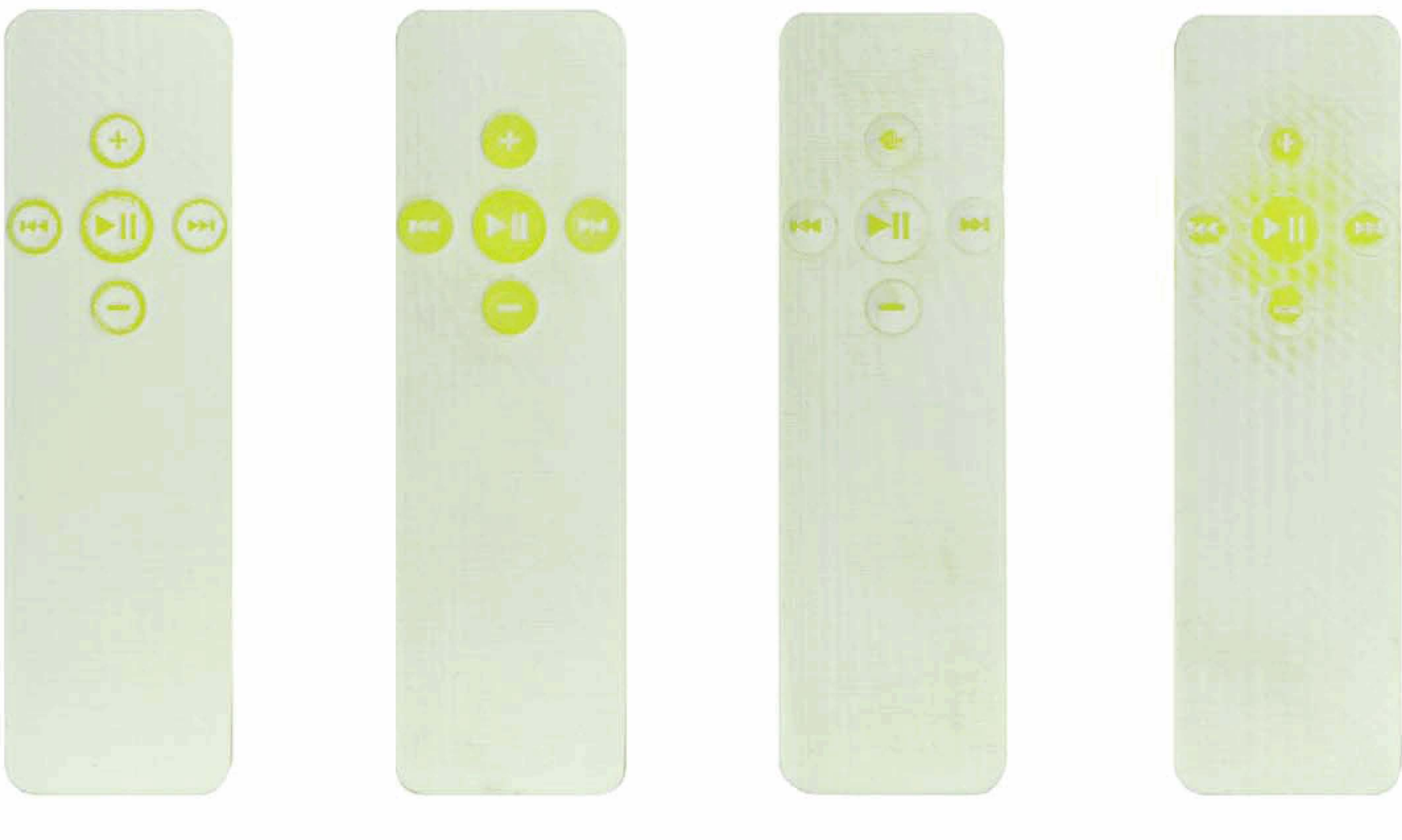

\# 4

\#4.1

\# 4.2

$\# 4.3$ 
Observations

The use of shading on the drawings allowed limited 3D shapes to be created (ref sample \# 4). This series of concave and convex buttons were trialled to recreate the forms of an earlier experiment (ref samples \# $2.25-2.40$ ) at a smaller scale and with a different wear mechanism (finger).

The removal of large areas with the laser cutter created a surface texture that was neither deliberate nor desirable (ref sample \# 4.3). The potential for poor craft through computer generated products emerged.

The painted (acrylic lacquer) finish applied to these samples lacked the gloss and precision of the "Apple Shuffle" on which they were based. The goal of creating products that remain desirable as they wear does not diminish the necessity for products to be highly desirable when new. Thus further research was required to improve the initial surface finish quality of the samples. 
5.9 Wear Bias 
Experiments: \# 5 - 5.1

Materials: White paint (5 microns) / White and yellow plastic (Acrylic)

Primary design issue: The use of matching or contrasting colours to conceal or reveal wear 

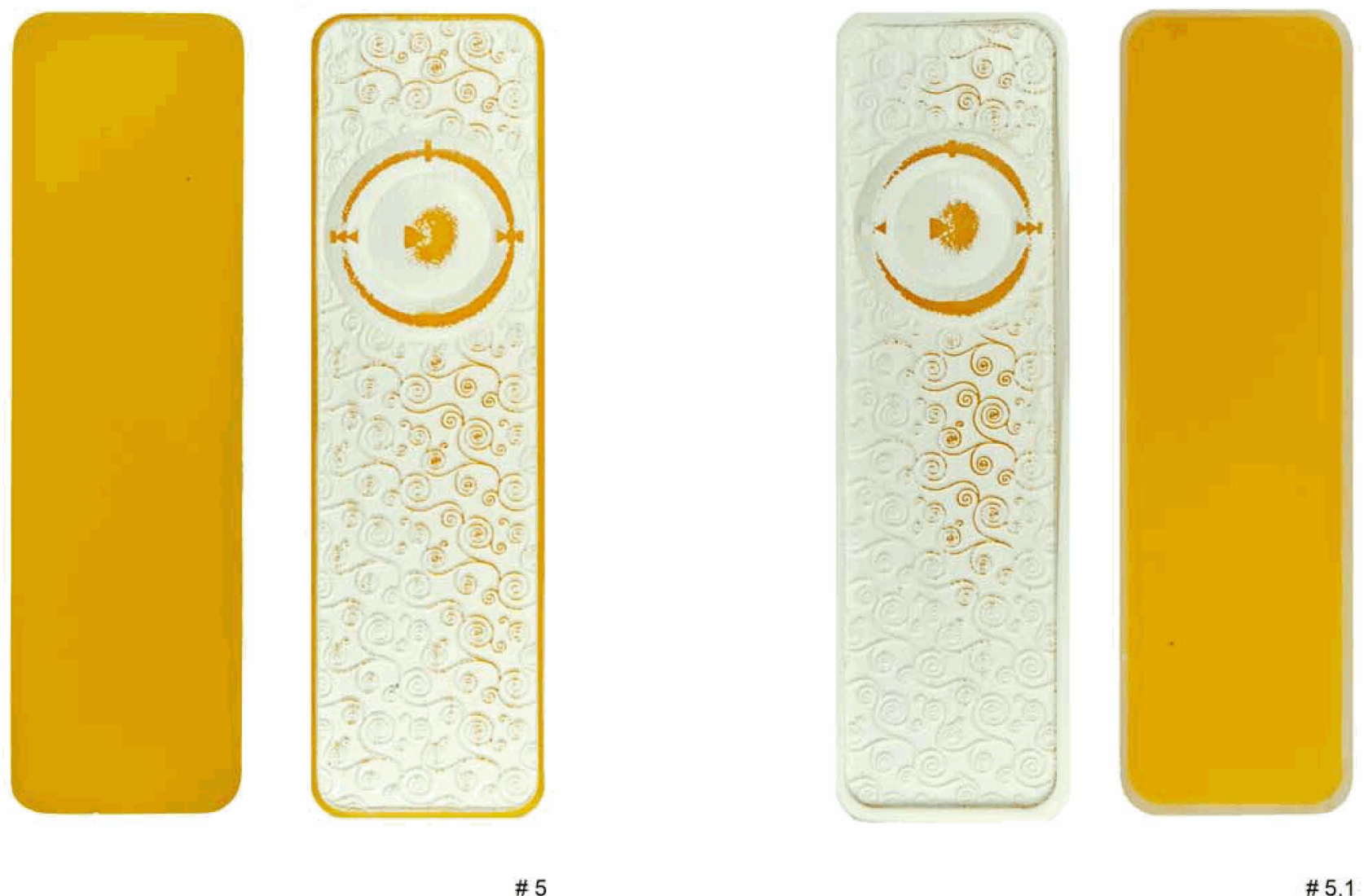

\# 5.1 


\section{Observations}

Distinguishing between the general actions that cause wear on the edges of products and the deliberate actions that cause wear on controls allows a bias to be created in the design. Through the use of exterior colours that match or contrast with the base material, the wear from each action can be visually highlighted (ref sample \# 5) or diminished (ref sample \# 5.1).

The multiple coloured sample (ref sample \# 5.1) was produced with a solid white plastic bezel around the edge of the product. The production technology to co-mould multiple solid colours in products is common creating a mechanism to allow designers to distinguish between wear patterns and the associated meaning derived from the action that created them.

A scroll patterns was used to reduce the visibility of the undefined and undesired surface texture created by the laser cutter over large areas. As the product became worn the top edge of the scroll was revealed as a fine, precise and deliberate line. The creation of 3D shapes through the laser engraver causes a surface texture that diminishes the clarity of the graphic symbols. 
5.10 From 2D to $3 \mathrm{D}$ 
Experiments: \# 5 - 5.1

Materials: Red paint (5 microns) / White plastic (Acrylic)

Primary design issue: Wear qualities between 2D and 3D 

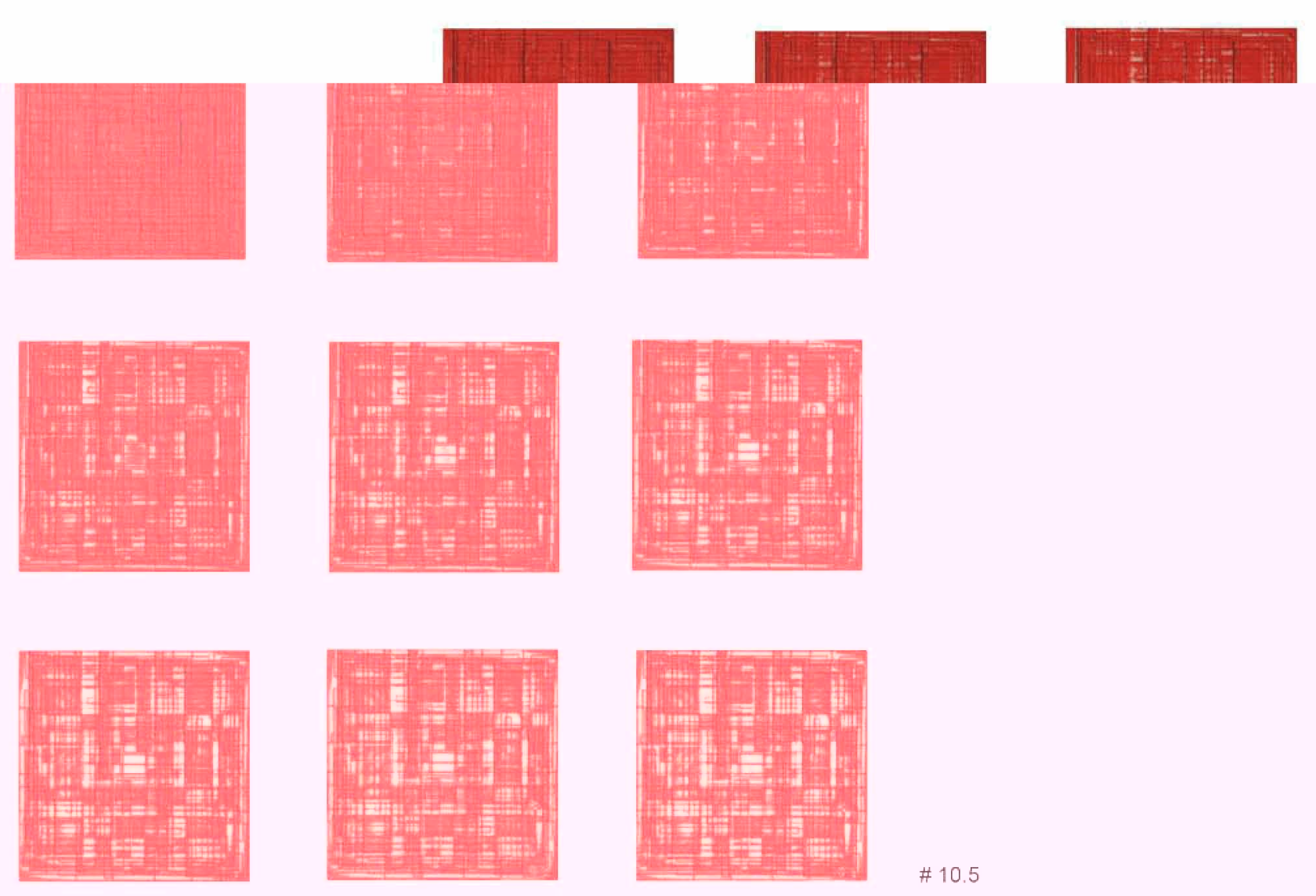
Observations

This sample was created to explore the potential of the laser cutter to use graduated tones in the 2D image to create surfaces with $3 \mathrm{D}$ qualities. While it reveals greater depth variation than the earlier monotone graphic images, the technique is limited in achieving complex $3 D$ forms. Further investigation is required to establish a manufacturing technique that offers the repeatability and flexibility of laser engraving with greater 3D

capacity. 
5.11 Growing 3D (Rapid Prototyping) 
Experiments: \# 14 - 16.3

Materials: Black paint (5 microns) / Plastic (ABS) Fuse desposition models (FDM)

Primary design issue: The potential of rapid prototyping technologies to create diverse products 


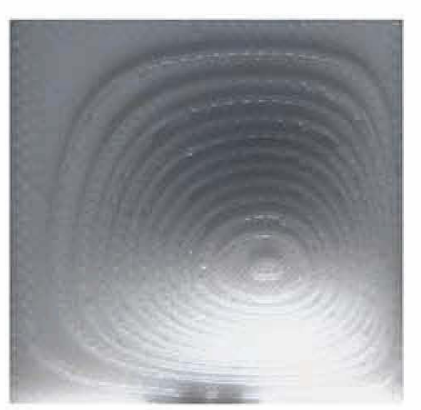

\#14

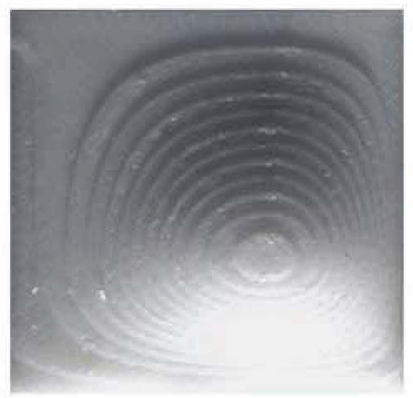

\# 14.1

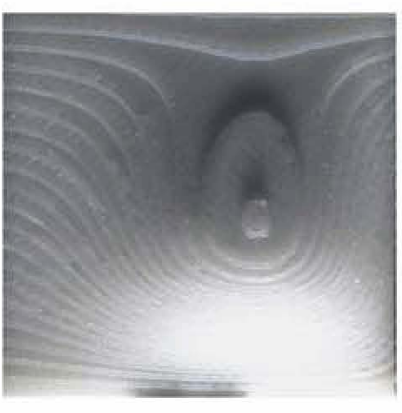

\#14.4

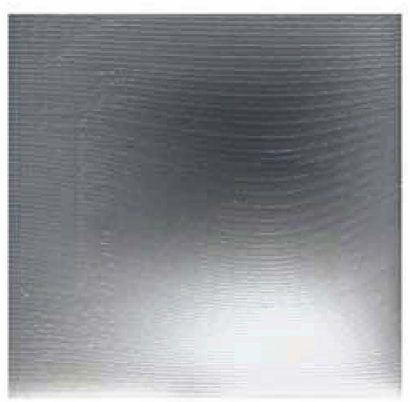

\# 14.8

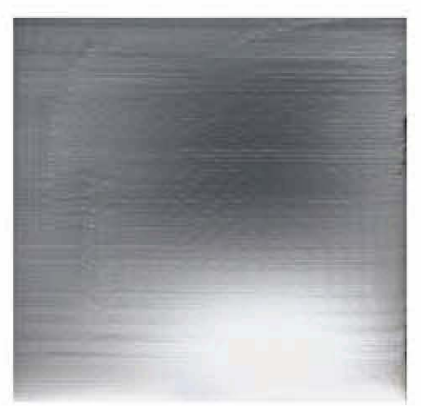

\# 14.9

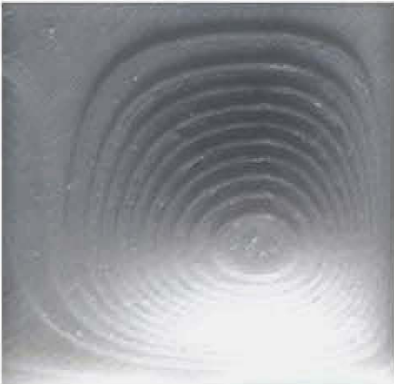

\#14.2

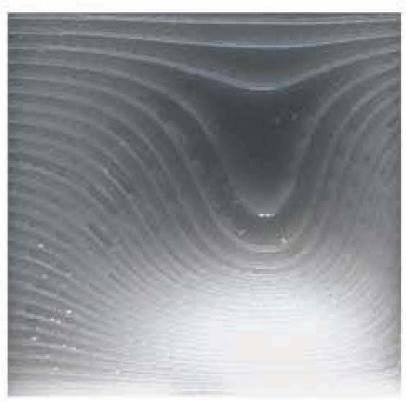

\#14.6

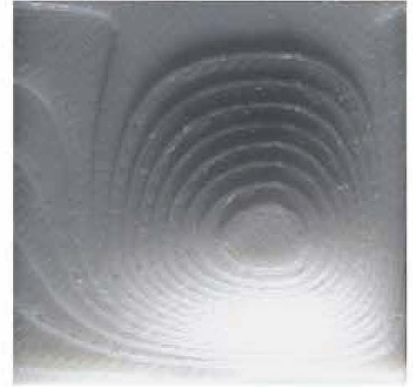

\# 14.3

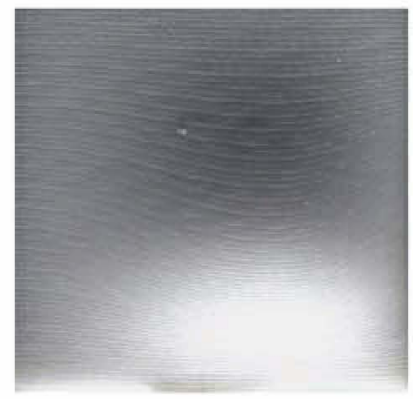

\#14.7 

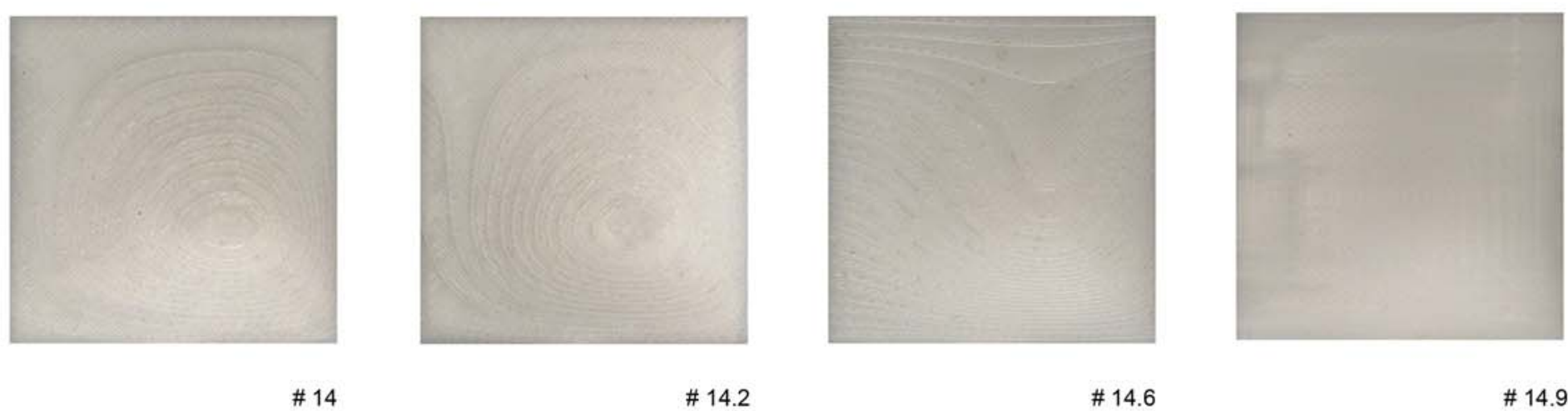

\# 14
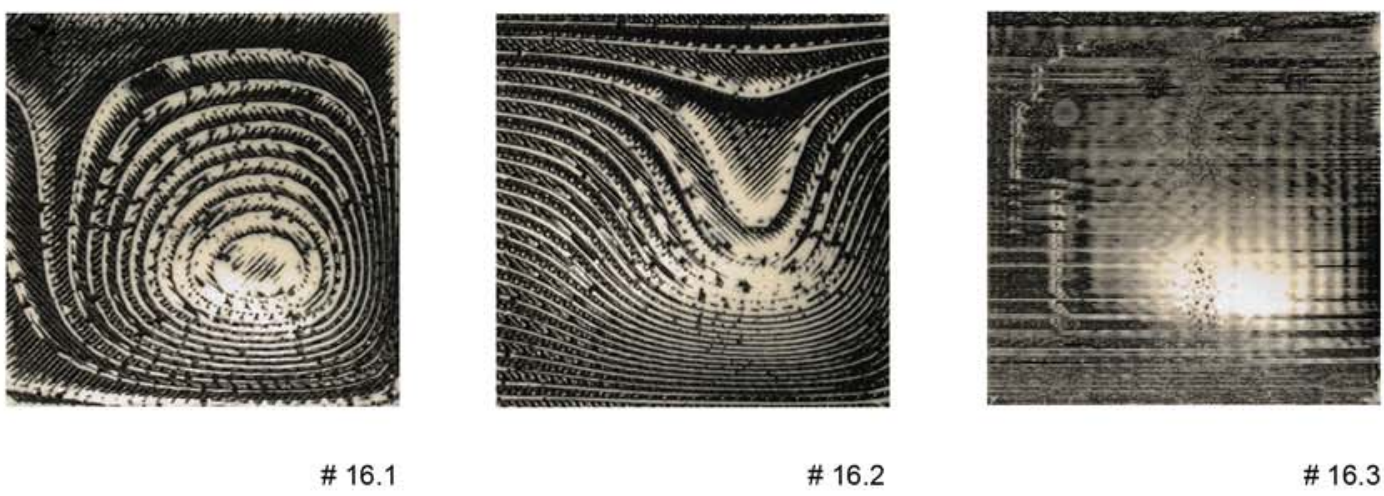

\# 16.3 


\section{Observations}

Rapid prototyping technology offers similar flexibility to laser cutting because it is computer controlled. The program used to create these samples (Solidworks) required greater operator skill than the simpler 2D programs used to create the laser cutter artwork hence a specialist was used to generate the CAD models. The potential to create complex 3D models allowed the wear experiments to extend beyond the emerging limitations of the 2D laser engraver.

The lines in the surface of these rapid prototyped samples expose the path taken by the machine during the manufacture of the product (ref samples \# 14-14.9). As such they offer a clear history about how the object was made and the sequence of the manufacturing process. By coating the sample with a contrasting exterior layer and then wearing back through the surface the initially subtle construction lines become highlighted (ref samples \# 16-16.3)

The digital file used to create these samples was identical. The variations in surface textures are derived from the manufacturing process that builds $3 D$ shapes from fine $(0.3 \mathrm{~mm})$ layers. By building the samples on different angles, the layers are modified creating different surface textures. The layering process creates a decorative pattern over the surface of the product that is inherent in the process not an additionally applied decoration. From this a new understanding of a machine aesthetic emerges that relates to the structure and growth patterns of organic objects such as plants and shells. Like these organic objects, the lines evident in the FDM products reveal the process of growth that links the object to a time continuum that can be continued into the process of use and eventual decay.

For a more complete description of this experiment and the issues related to it, refer to chapter 4 
5.12 Visual Variation 


\section{Experiments: \# 17 - 76}

Materials: Oxidised brass / Brass (15 microns) / Zinc casting

Design issue: The aesthetic qualities of oxidisation layers on reactive metals.

This experiment was undertaken to explore the potential of metal (brass) to react with the environment to create diverse and site specific coatings.

3D products were produced in a range of generic forms (circular, rectangular, curved and organic) to explore the relationship between form and surface coating. These products were developed using a computer modelling program (Solidworks) and were physically produced using a range of rapid prototyping technologies. A centrifugal casting process was used to consistently produce multiples of the same product and shape.

These product were electroplated with a 15 micron brass coating. To oxidise the product they were submersed in a solution of deionised water and liver of sulphur for 3 minutes to create a surface oxidisation.

Variations of these generic products were used in the following surface and enclosure experiments. 


$$
\text { MAMA }
$$




$$
\text { MAMAA }
$$




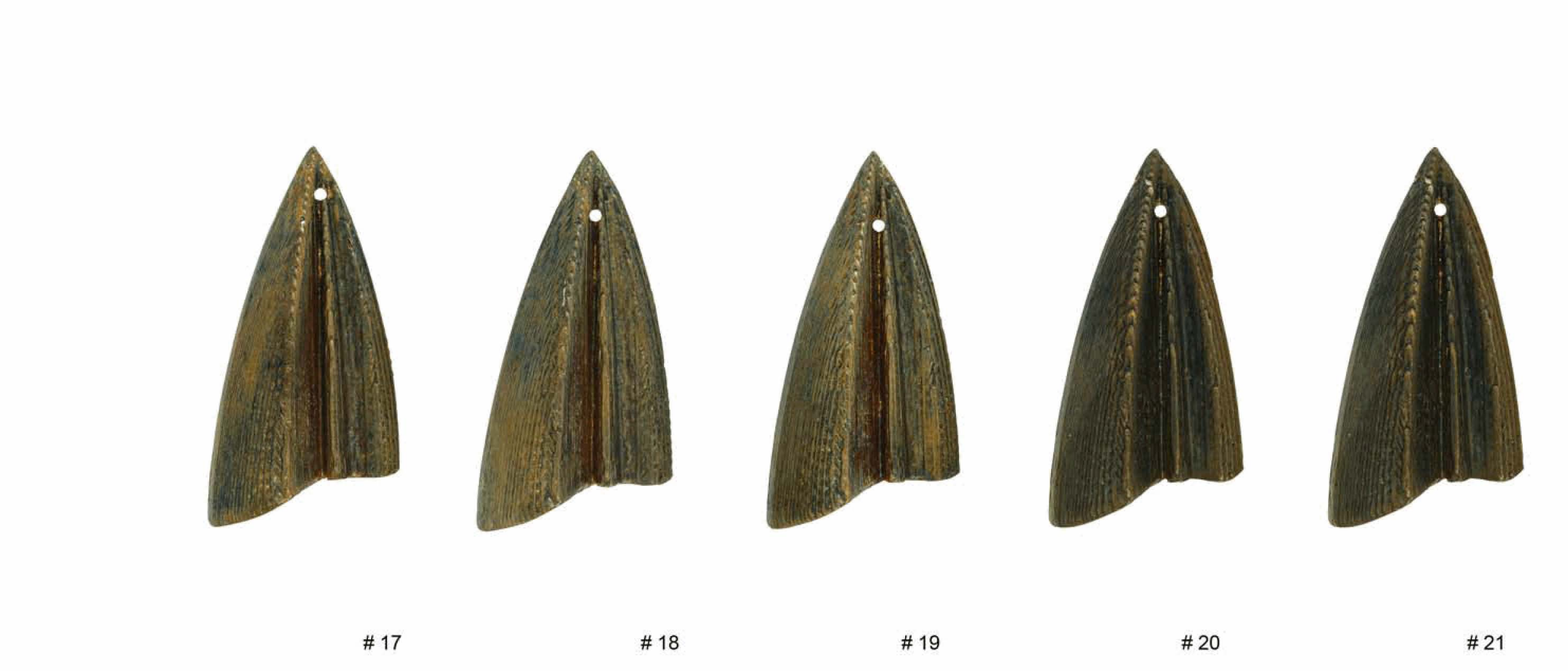




$$
\text { MAAMA }
$$




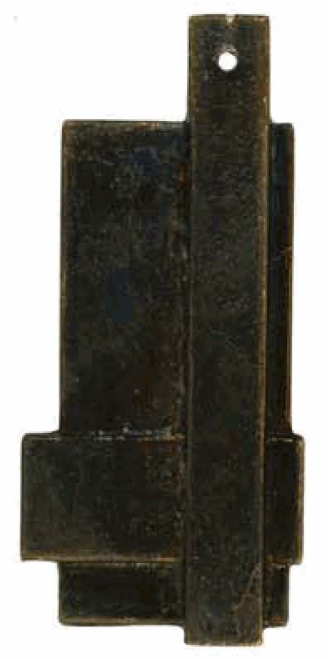

\# 67

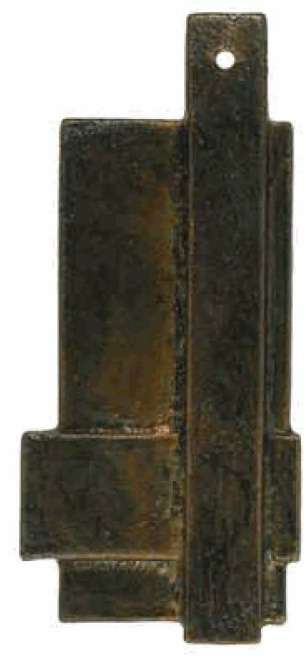

\#68

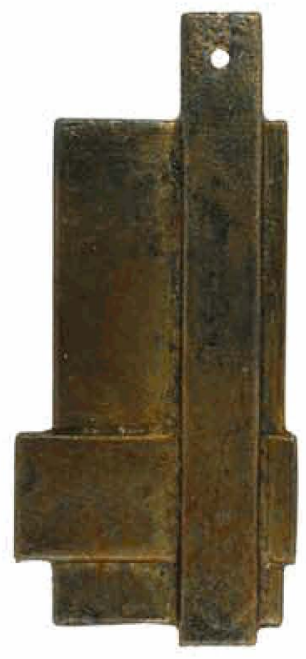

\# 69

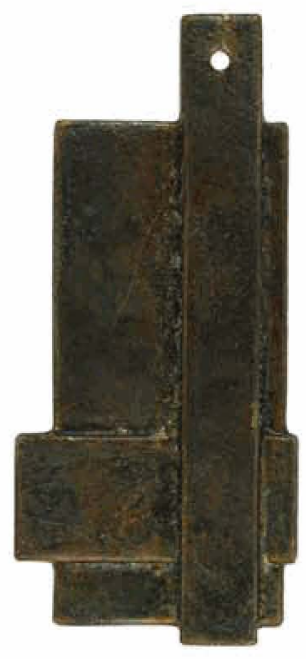

\# 70

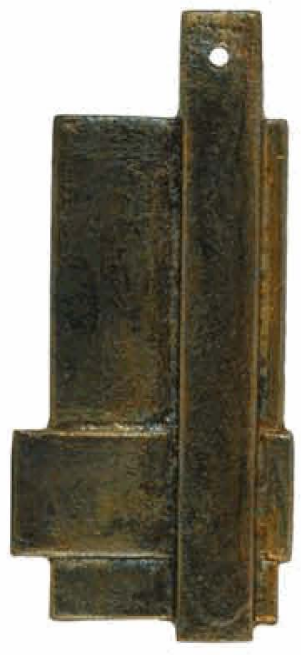

\# 71 


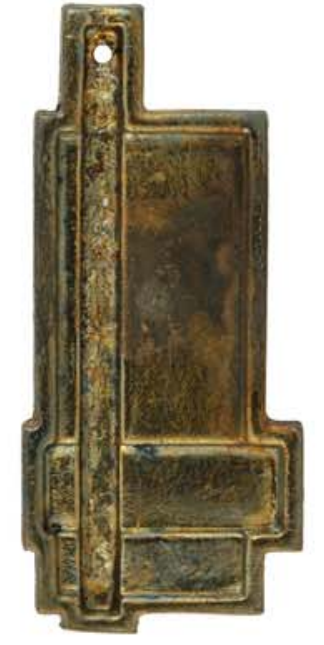

\# 72

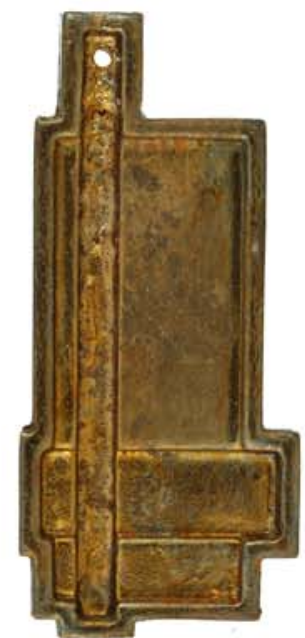

\# 73

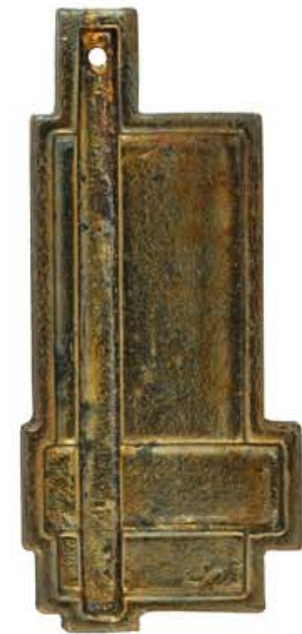

\# 74

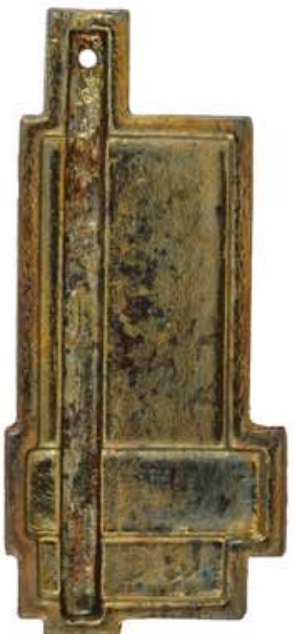

\# 75

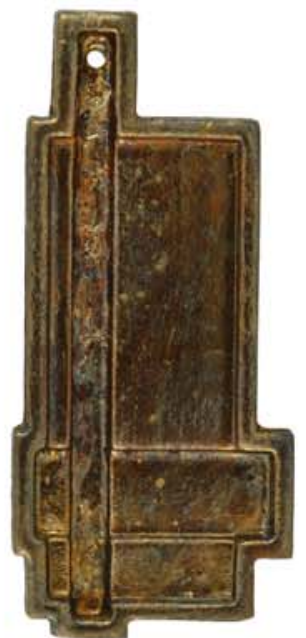

\# 76 


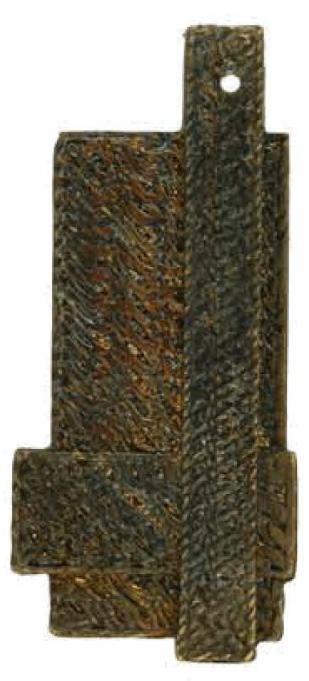

\# 57

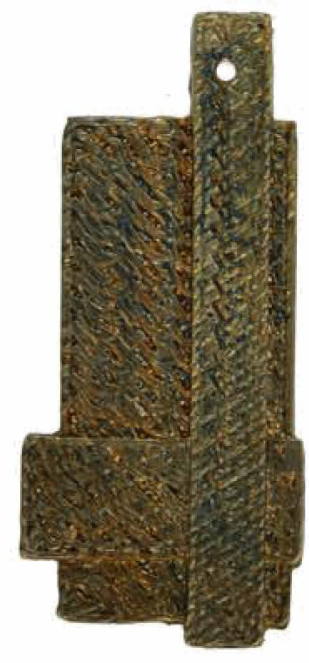

\# 58

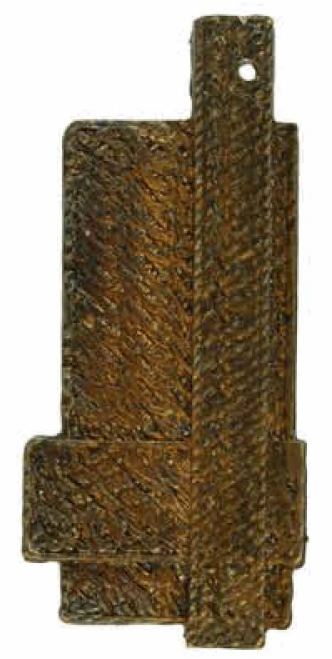

\# 59

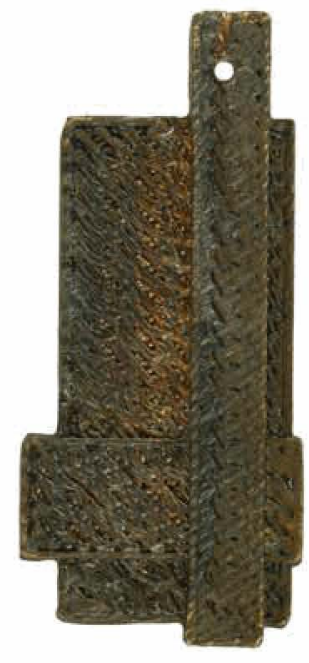

\# 60

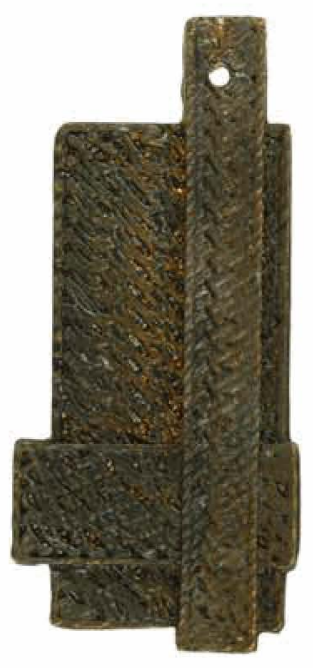

\#61 


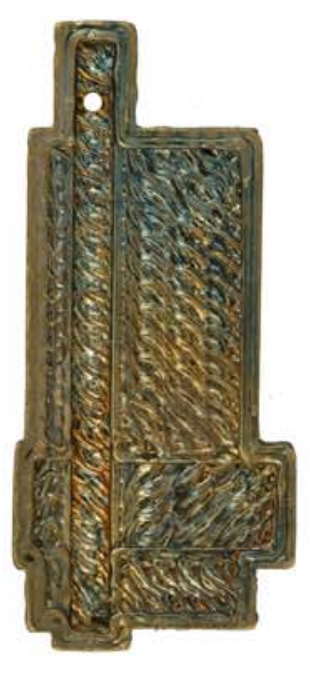

\# 62

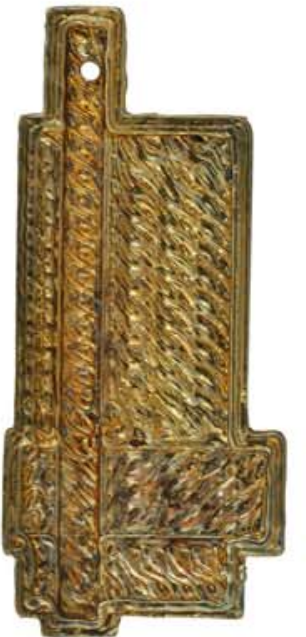

\# 63

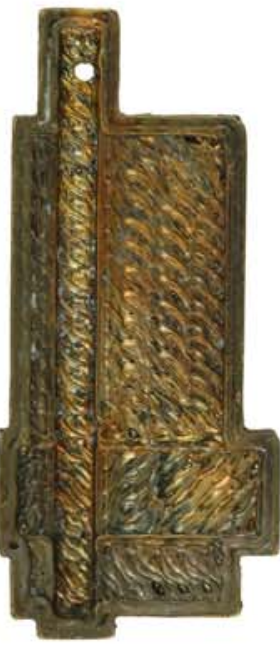

\# 64

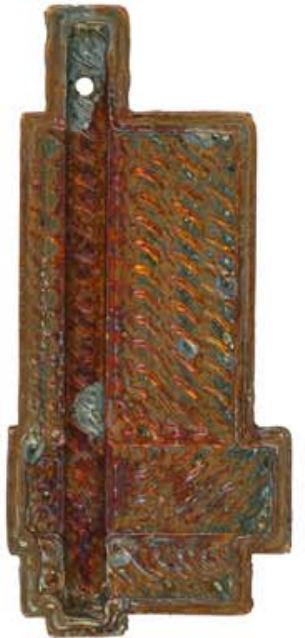

\# 65

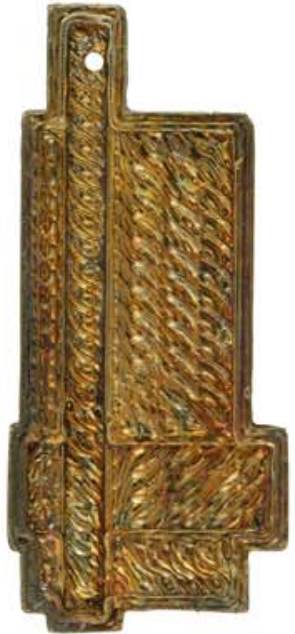

\# 66 

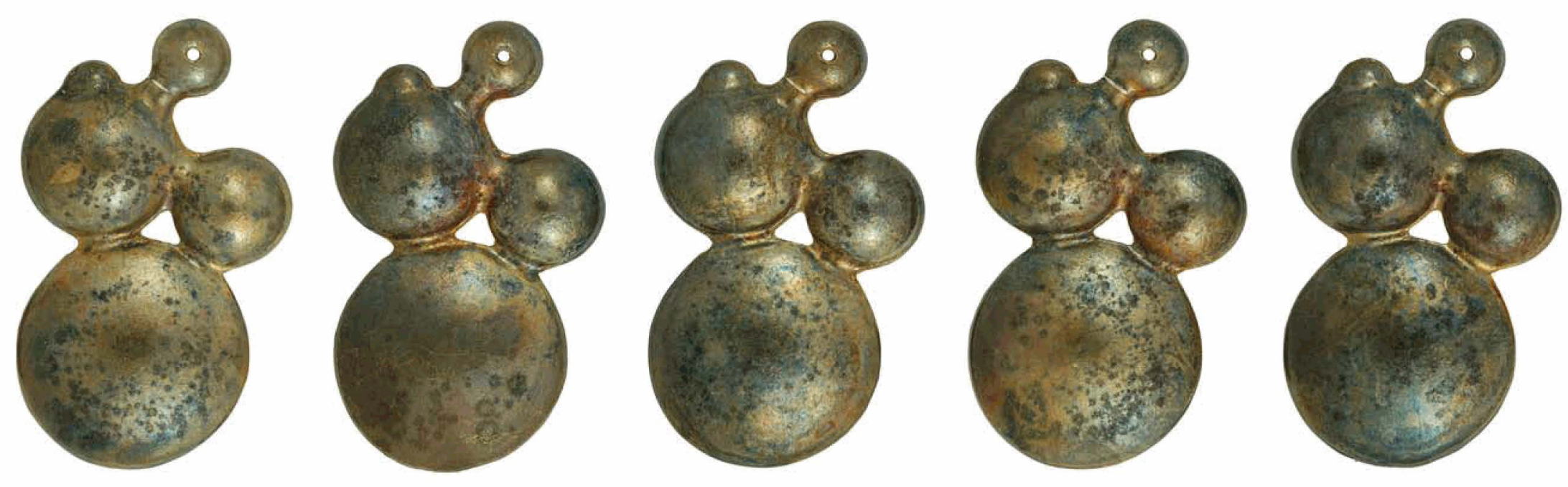

\# 47

\# 48

\# 49

\# 50

\# 51 

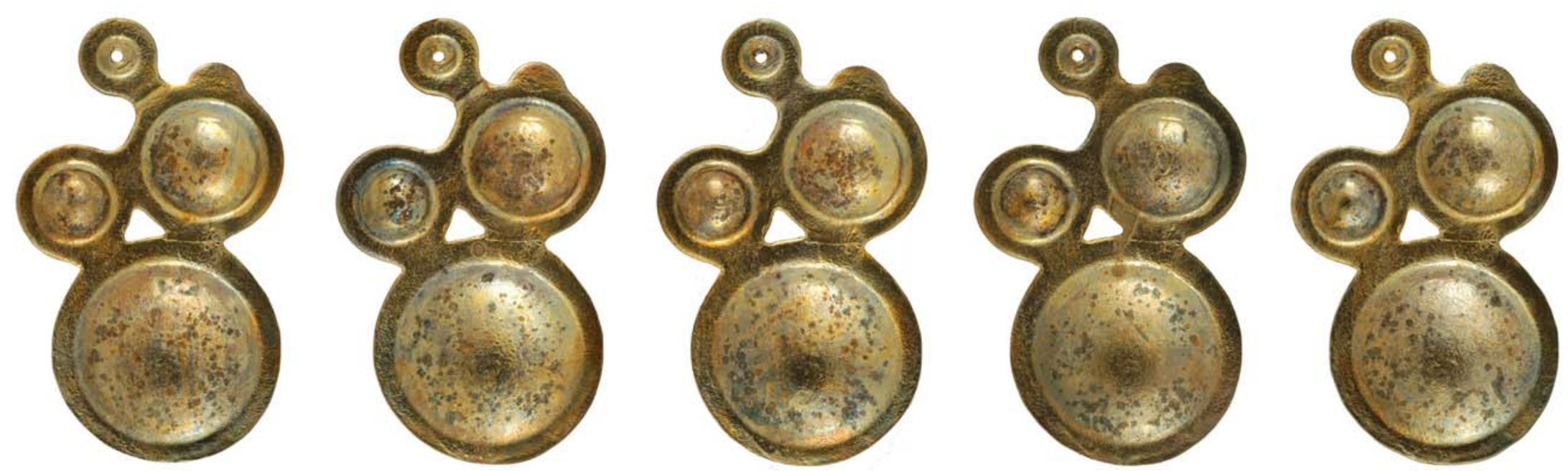

\# 52

\# 53

\# 54

\# 55

\# 56 


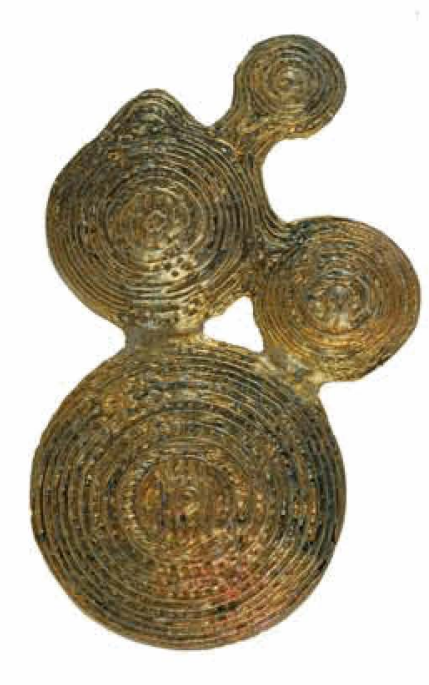

\# 37

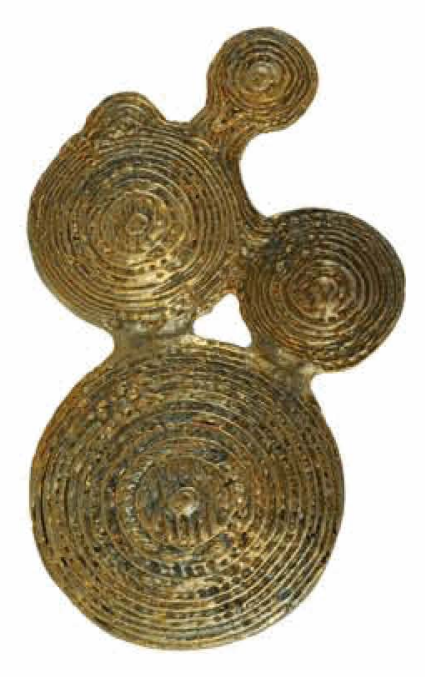

\#38

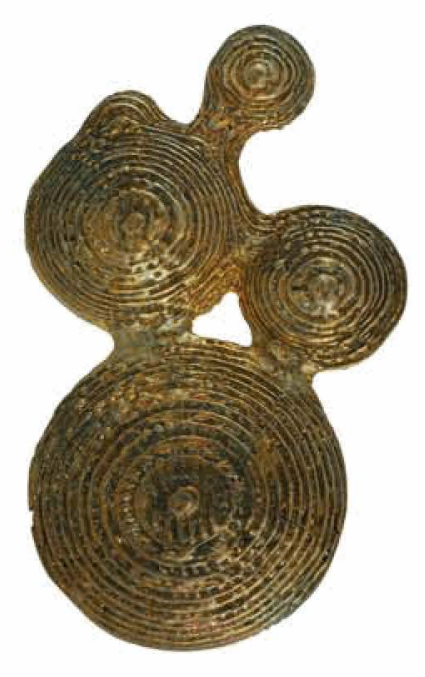

\# 39

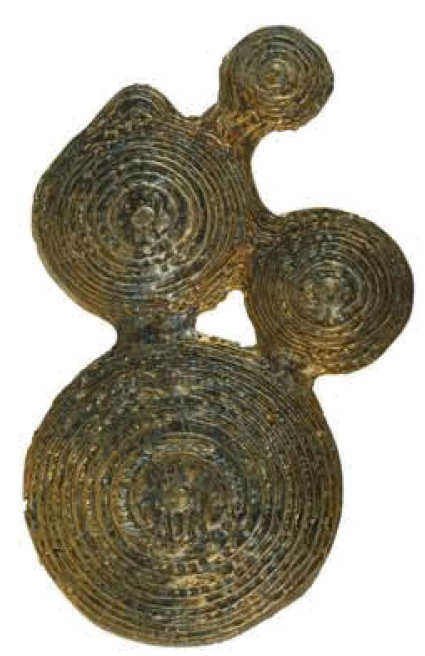

\# 40

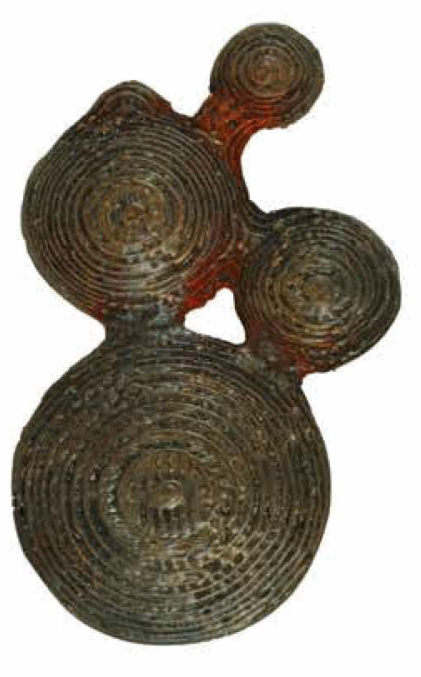

\#41 

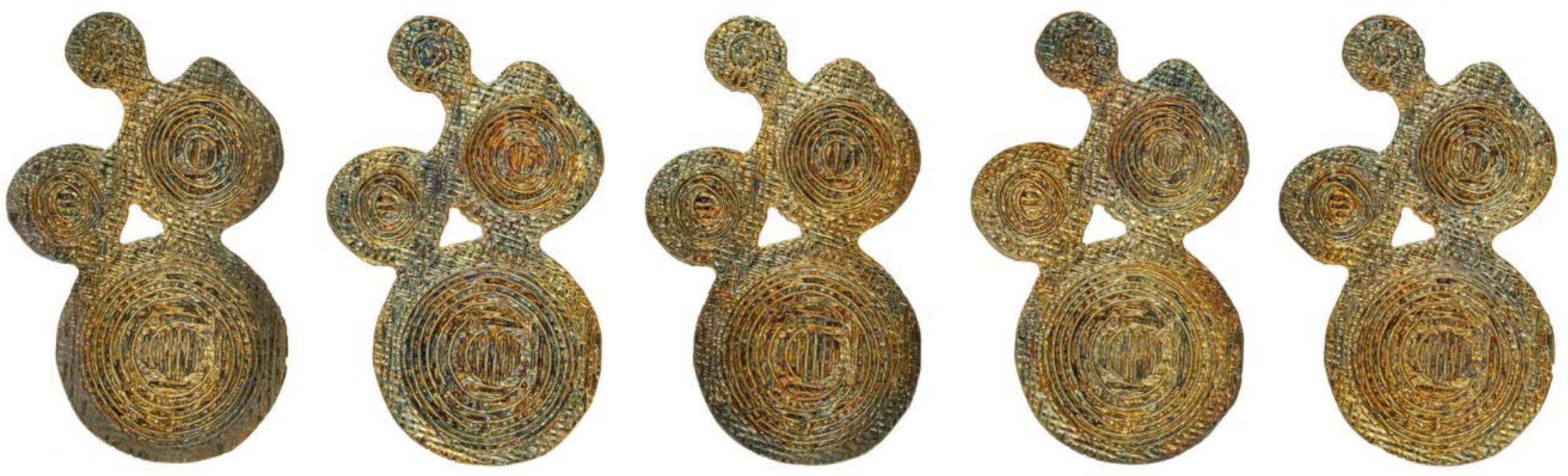

\# 42

\#43

\# 44

\# 45

\# 46 
Observations

Surface tension between the oxidisation fluid and the form of the product created variations in the resulting patterns that were unique yet influenced by the products shape (ref sample \# 68). This interaction between the product's form and surface pattern creates a discernible history, expressive of the fluid process used connecting the new product to a pre-established time continuum.

The minute thickness of the oxidation layer (<1 micron), while capable of modifying the product appearance, would not affect the product's functional performance. This distinction between appearance and performance would allow the creation of products with the design advantages of variation and unique character without diminishing the mass production requirements for uniform size tolerances necessary for interchangeable components.

While the size tolerance of the sample was unaffected by the oxidisation process the need for a visual tolerance is critical. This requires the designer to define and communicate a range of visually acceptable finishes rather than one ideal (ref sample \# 65). A level of interpretation is required during the manufacturing process that will require more sophisticated communication and visualising tools to initially establish the variations and then to set limits acceptable to the designer.

The texture on the samples created using the fuse deposition process (FDM) modified the surface tension of the oxidisation fluid developing small areas of varying oxidisation between the ridges (ref samples \# 37). This resulted in the oxidisation patterns being more disjointed and thus less discernible than the oxidisation patterns on the smooth samples. This suggested that the application of a complex and reactive finish needs to be considered in conjunction with the product's form and surface texture to achieve a balanced overall composition. 
5.13 Surface Wear (Table) 
Experiments: \# $77-156$

Materials: Matt black paint (5 microns) / Zinc casting

Design issue: The aesthetic qualities of wear that develops in 3D products when they interact with 2D surfaces.

This interaction occurs when products are placed down or moved across flat surfaces (common in furniture). The transition between these sites from the dynamic hand onto static $2 \mathrm{D}$ surfaces creates one of the critical wear mechanisms affecting portable and personal electronic products.

A consistent and non-reactive surface coating was applied over the zinc casting. The samples were synthetically worn using a flat abrasive disc and hand sanded with P600 wet and dry sandpaper. This created a non reflective metallic finish with a fine directional texture. 


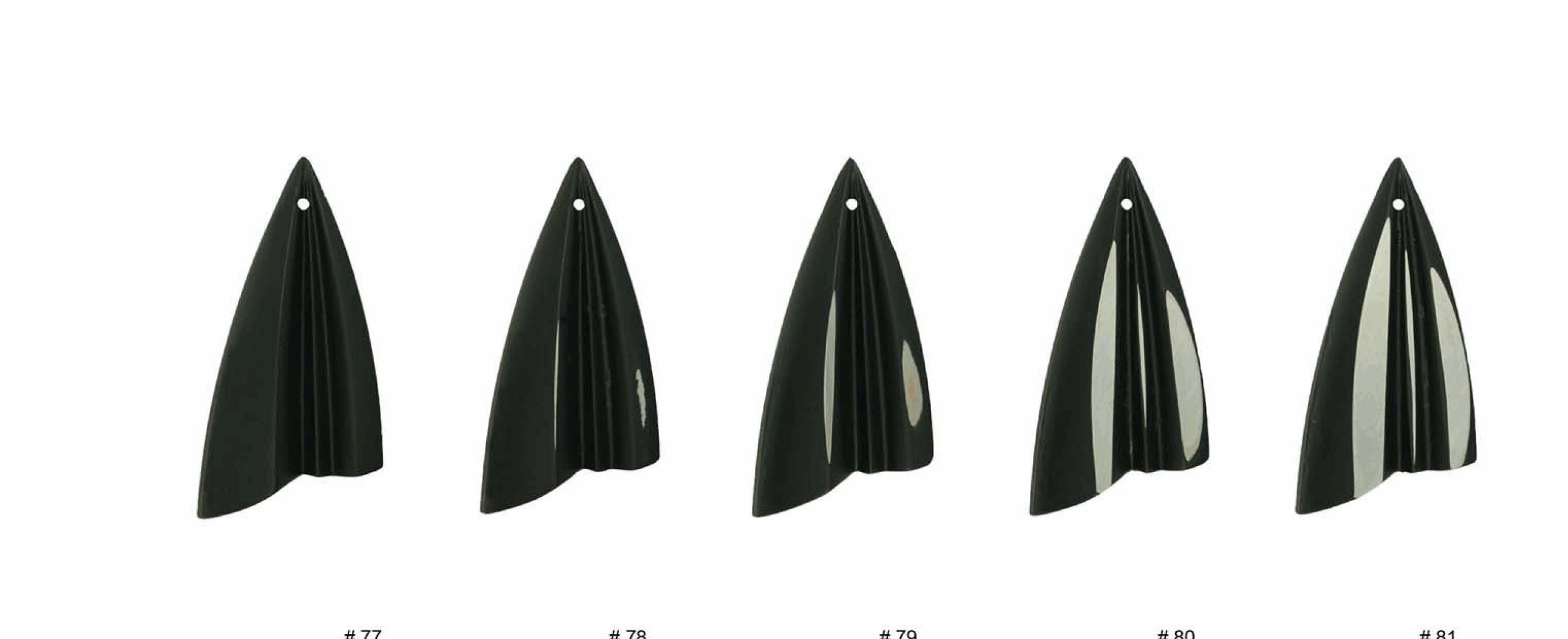



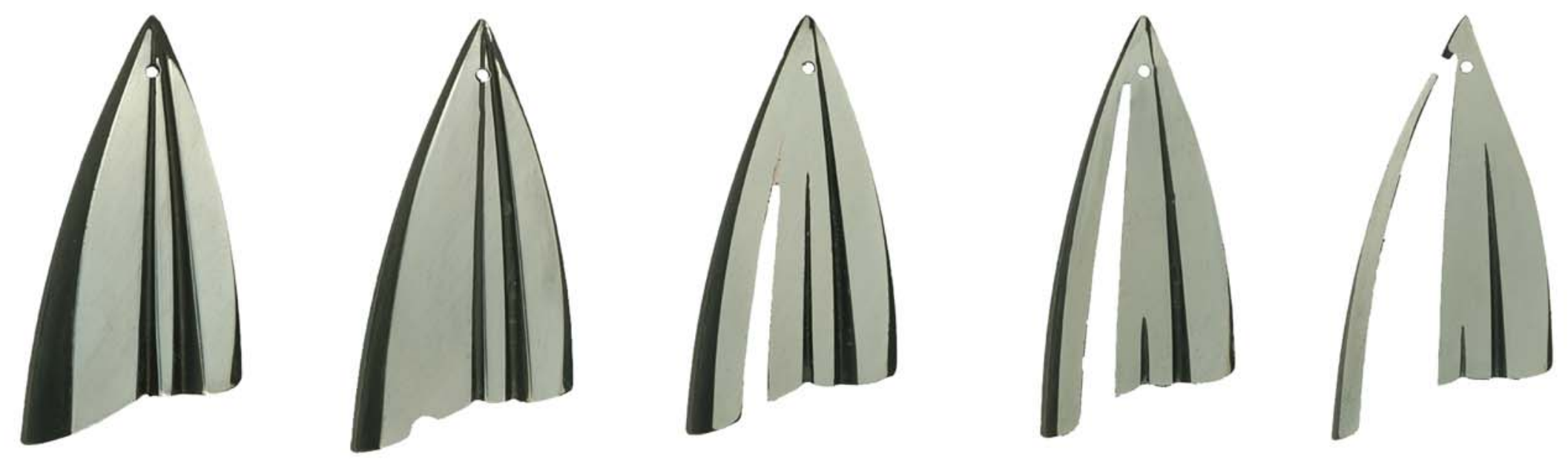

\# 82

\# 83

\# 84

\# 85

\# 86 


$$
\Delta \mathbb{\Delta a \mathbb { A }}
$$




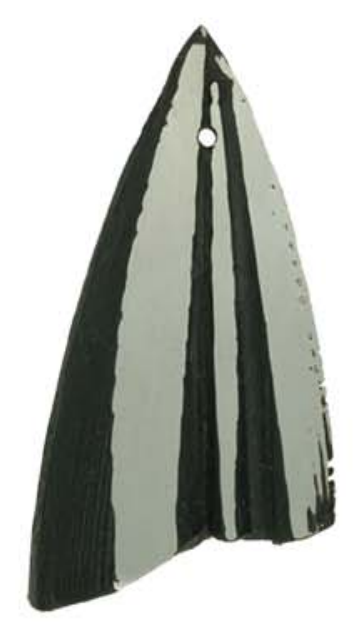

\# 92

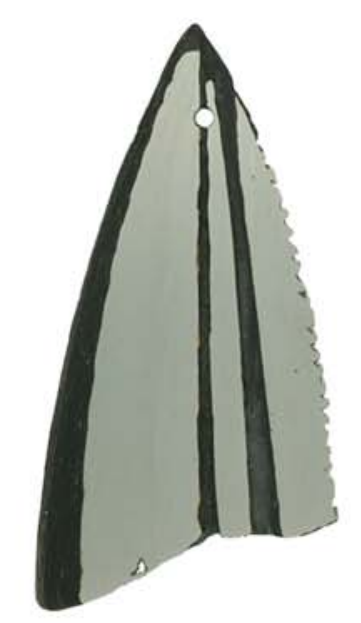

\# 93

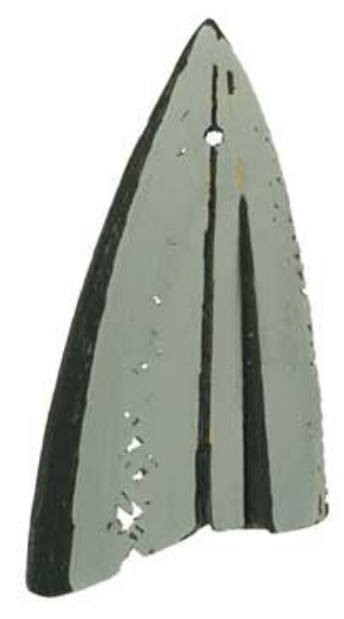

\# 94

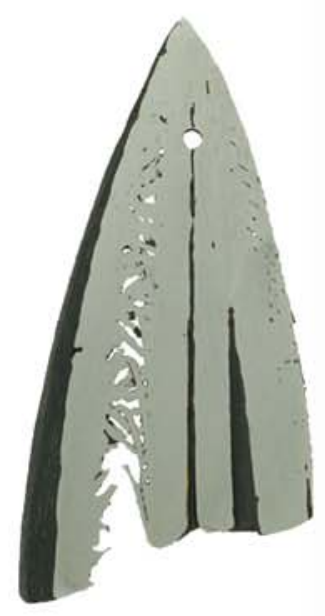

\# 95

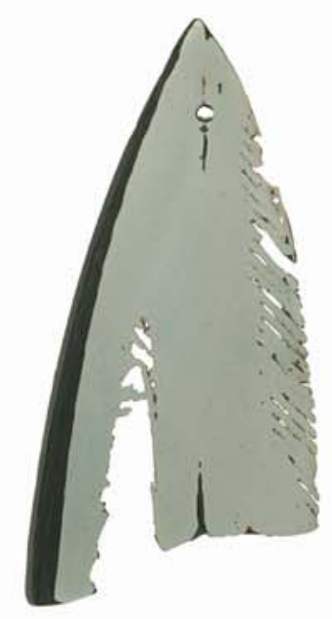

\# 96 


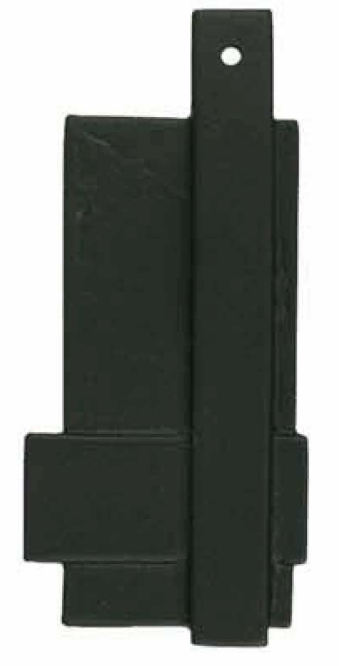

\# 97

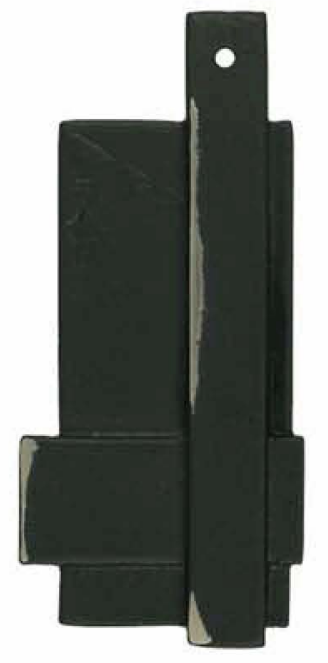

\# 98

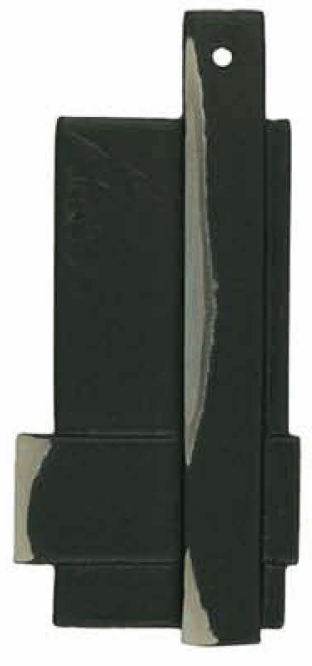

\# 99

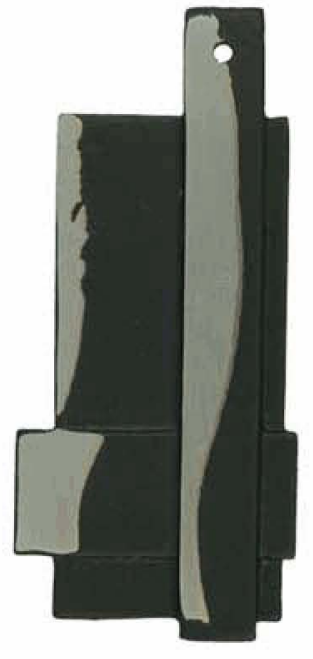

\# 100

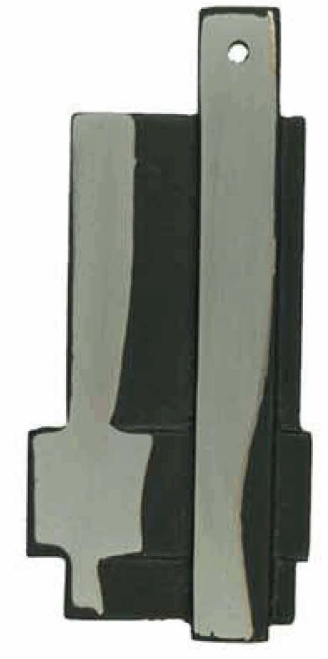

\# 101 

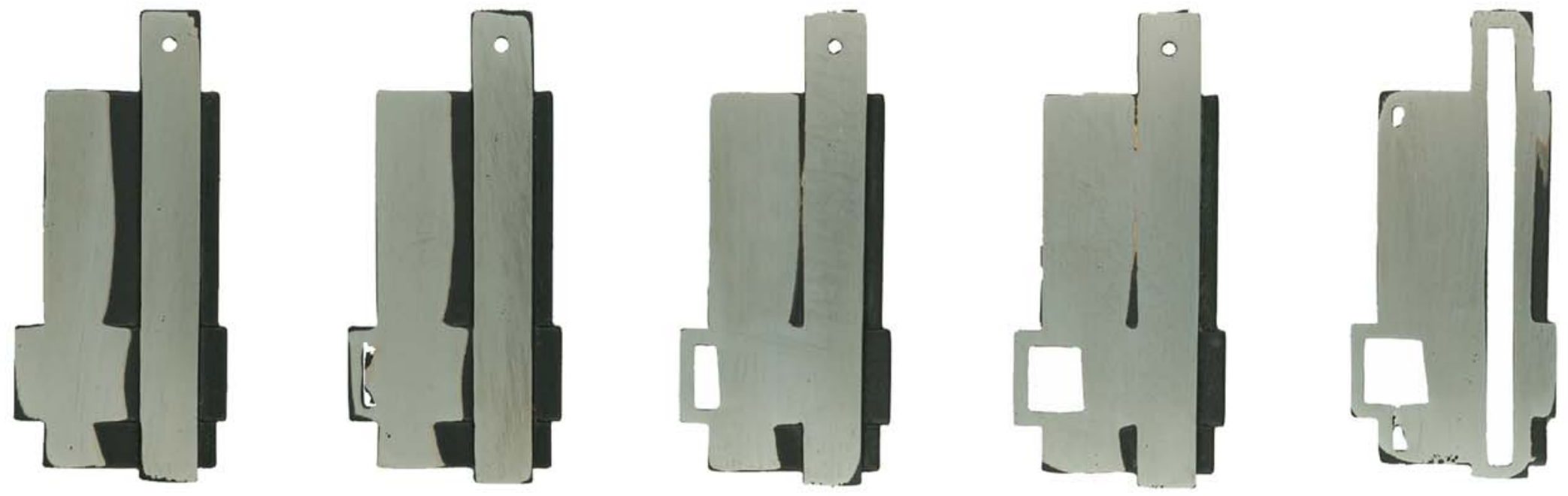

\# 102

\# 103

\# 104

\# 105

\# 106 


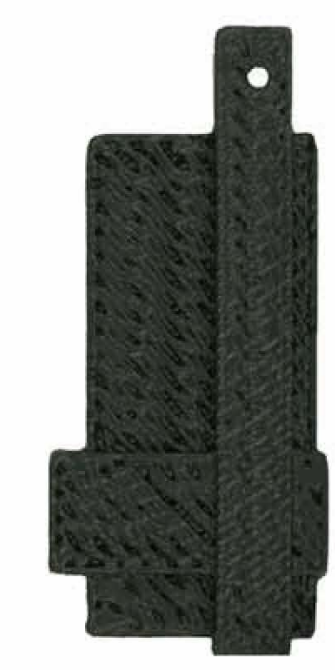

\# 107

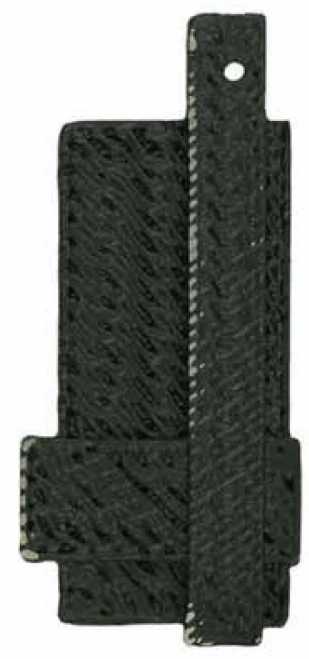

\# 108

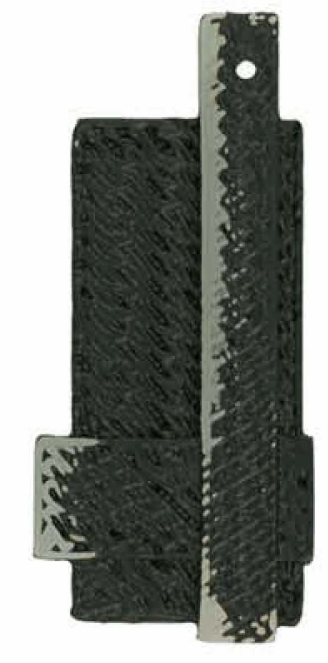

\# 109

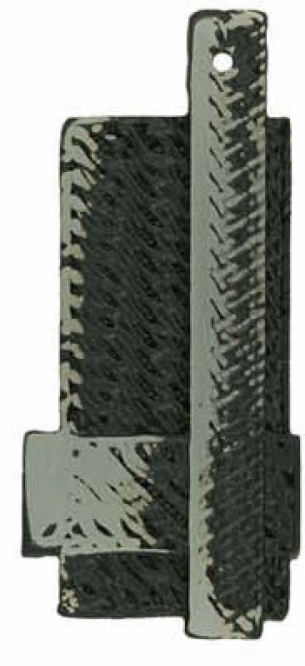

\# 110

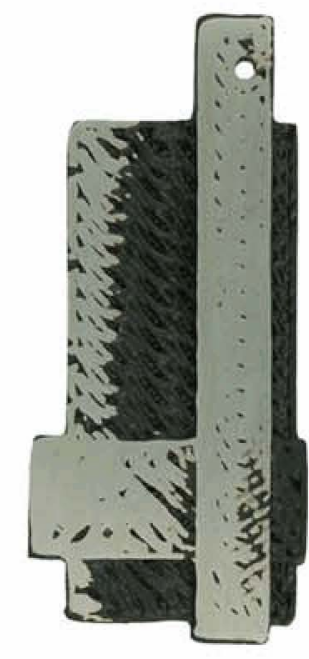

\# 111 


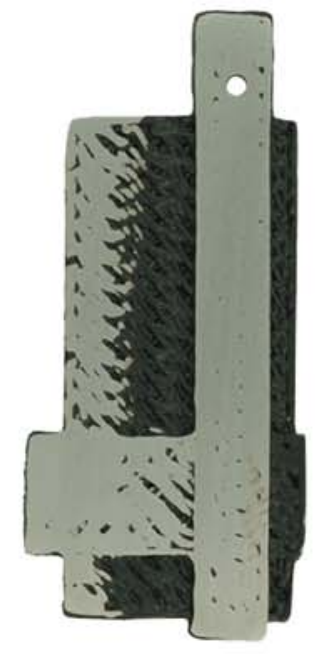

\#112

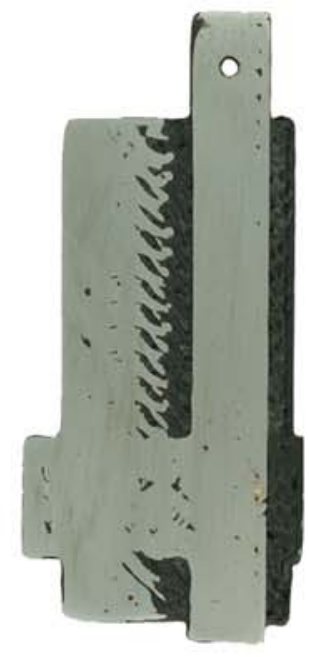

\# 113

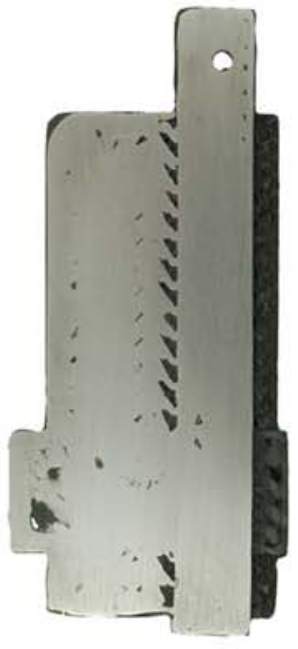

\# 114

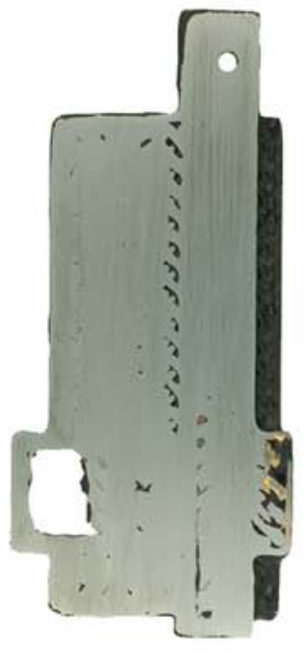

\# 115

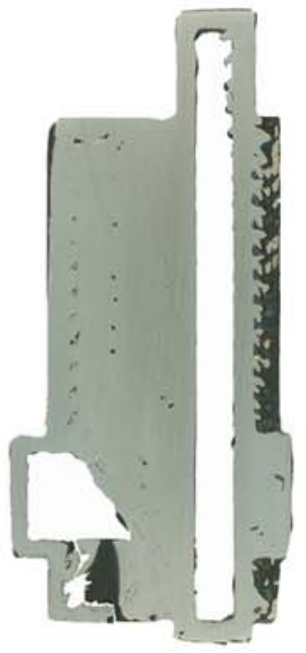

\#116 


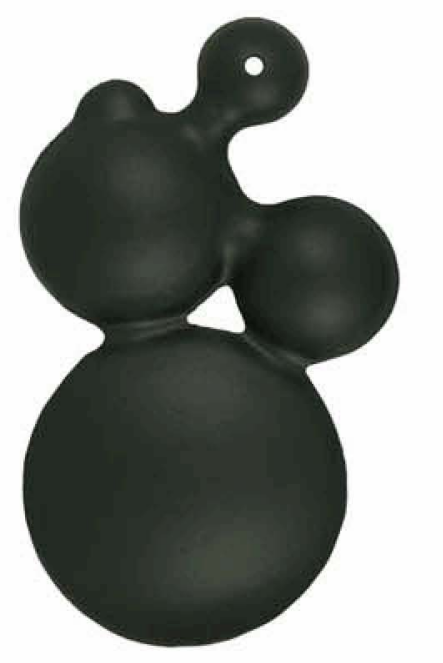

\# 117

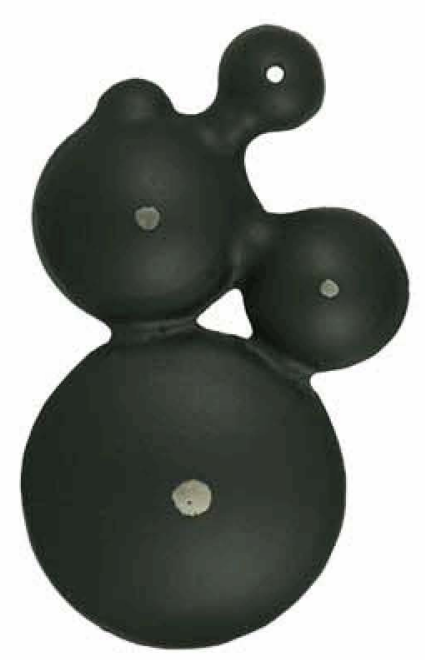

\#118

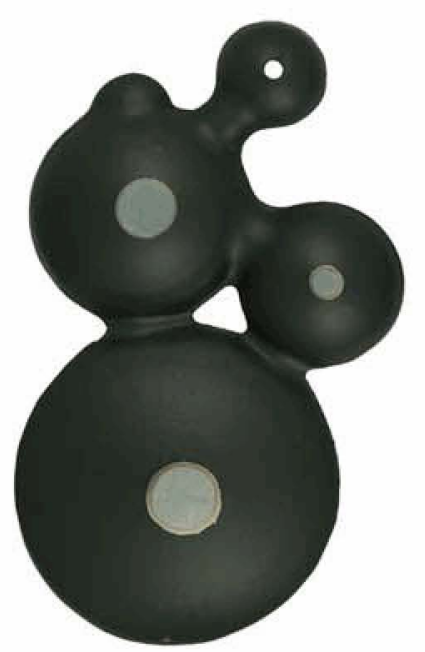

\#119

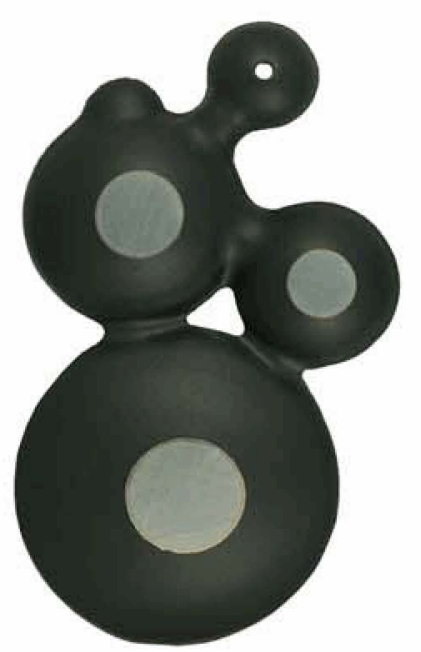

\#120

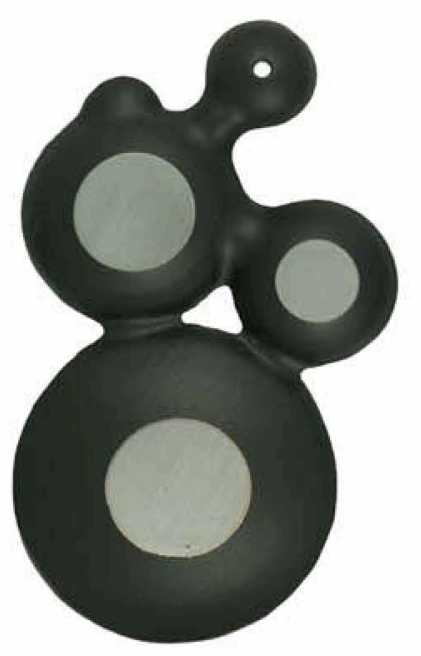

\#121 

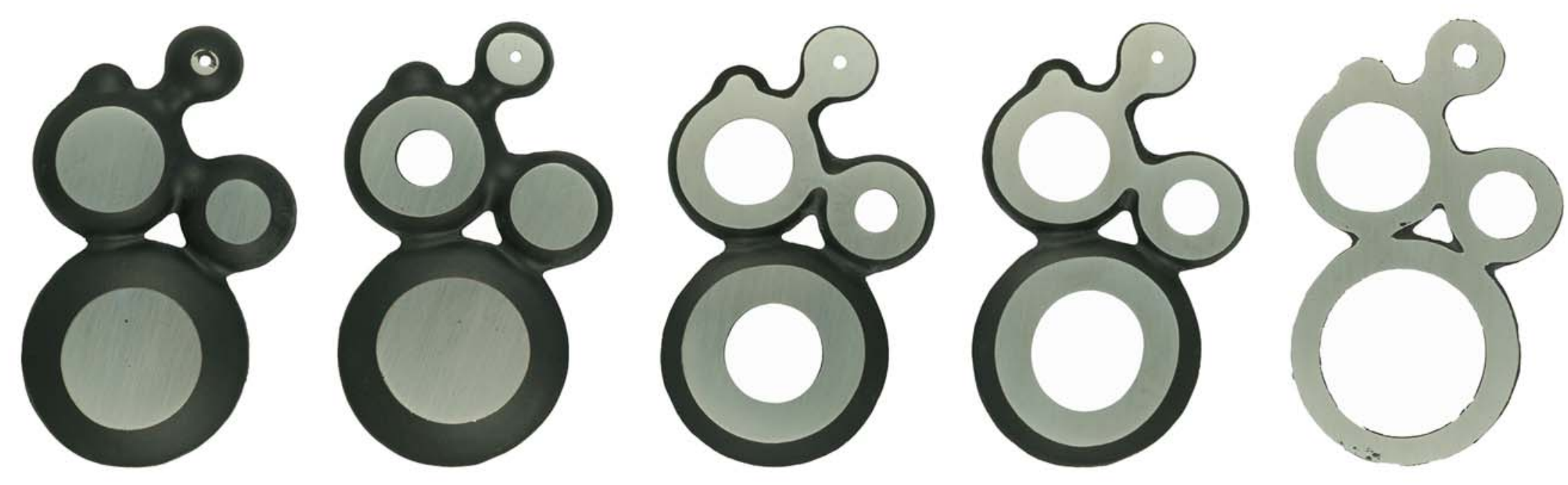

\# 122

\# 123

\#124

\#125

\#126 


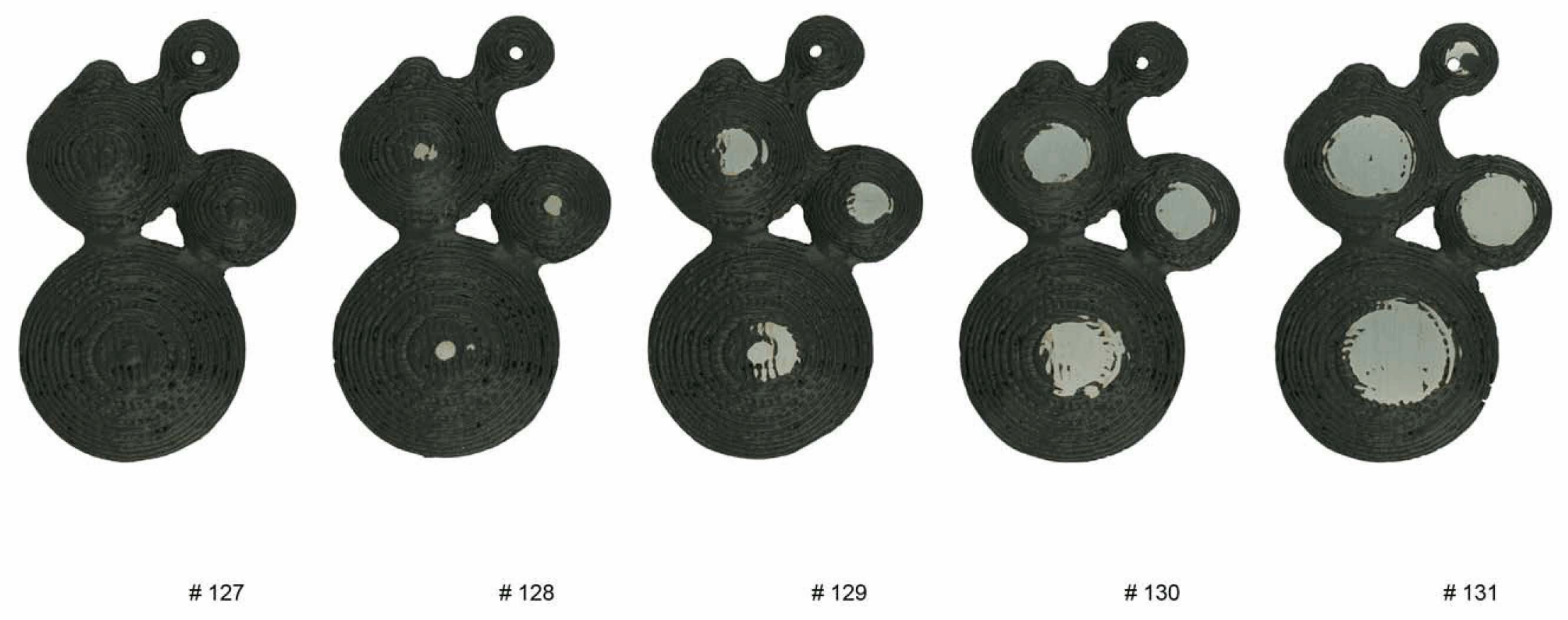




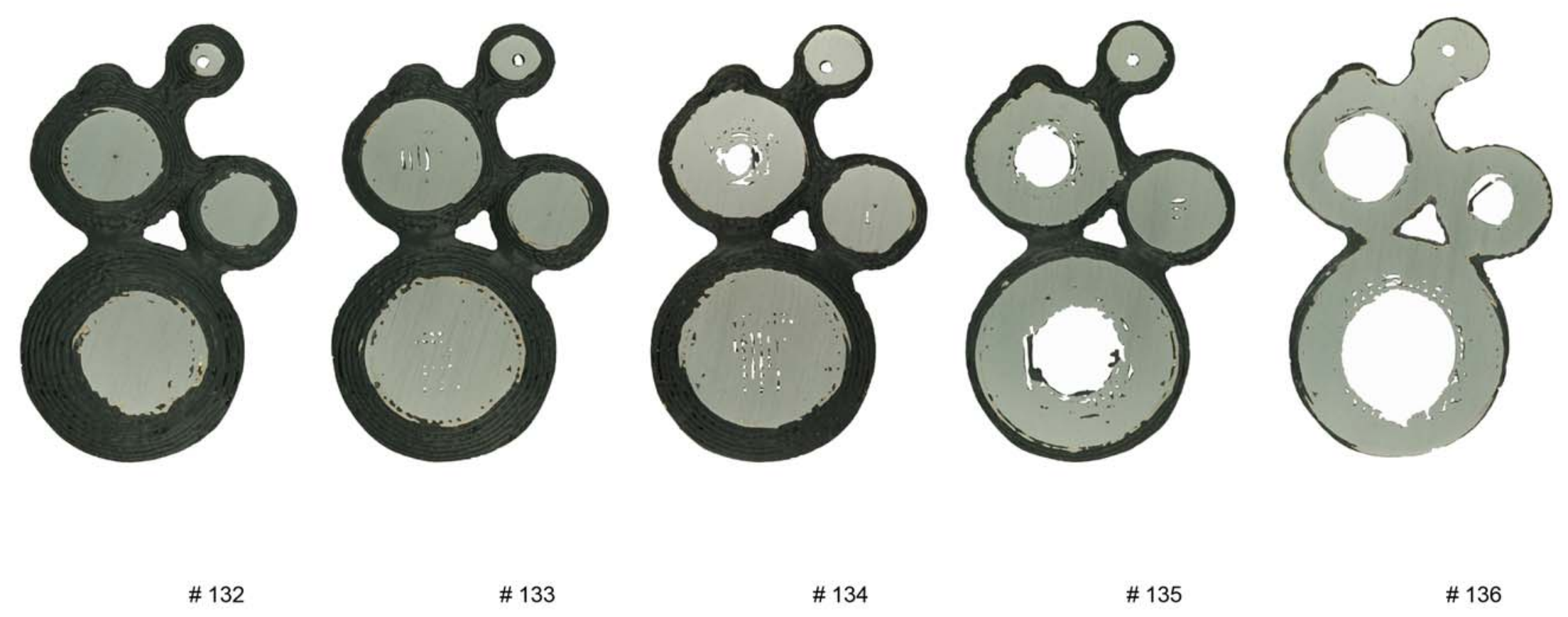




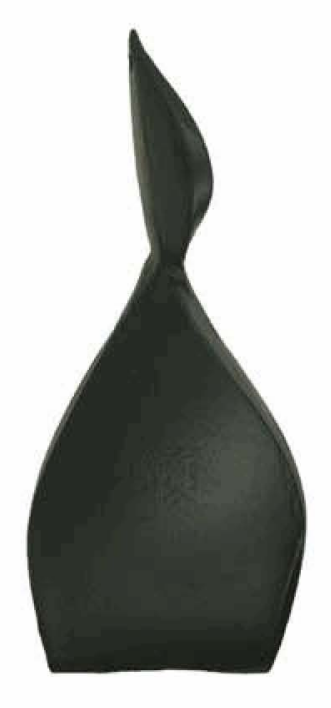

\# 137

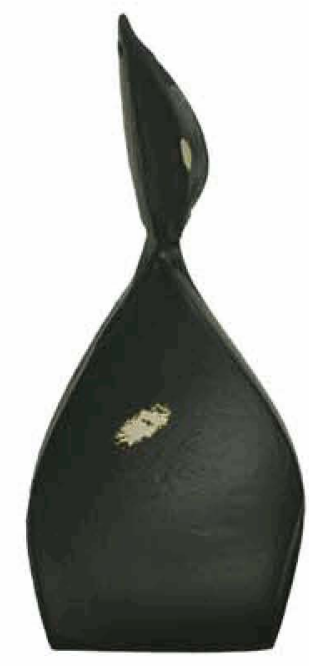

\#138

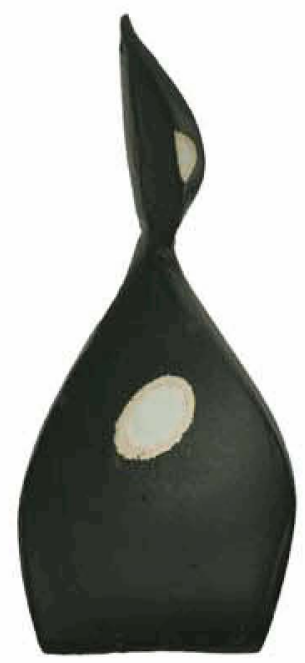

\# 139

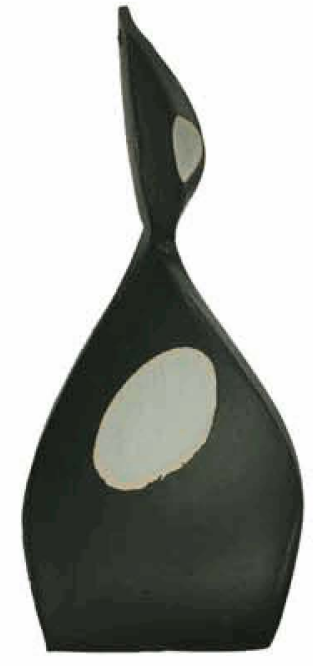

\# 140

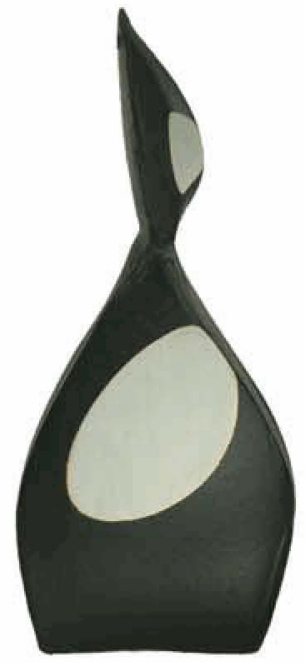

\# 141 


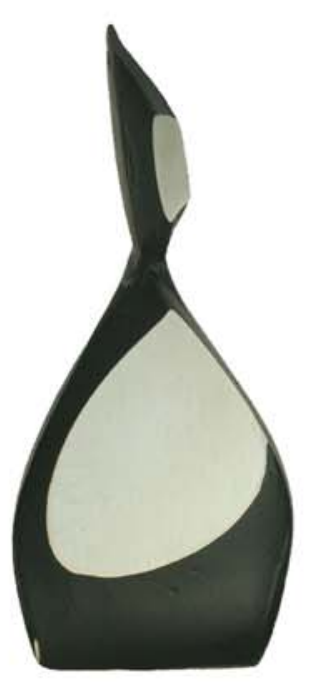

\# 142

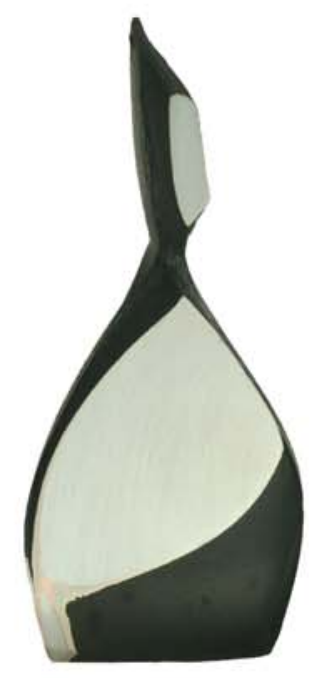

\#143

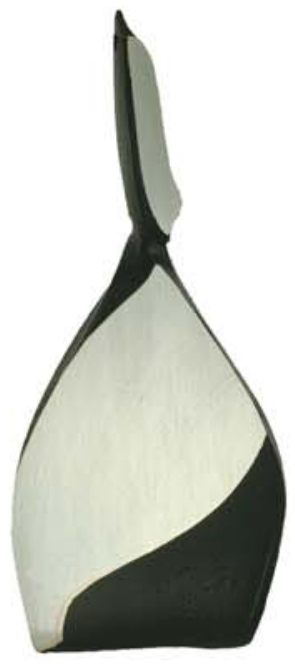

\# 144

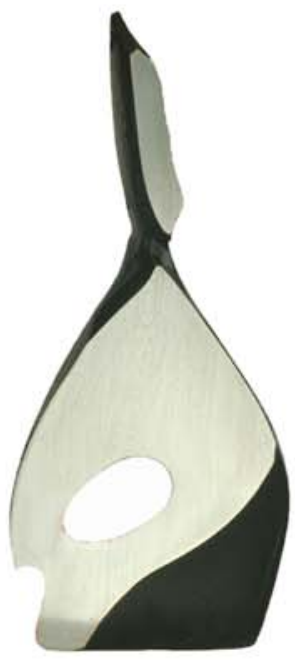

\# 145

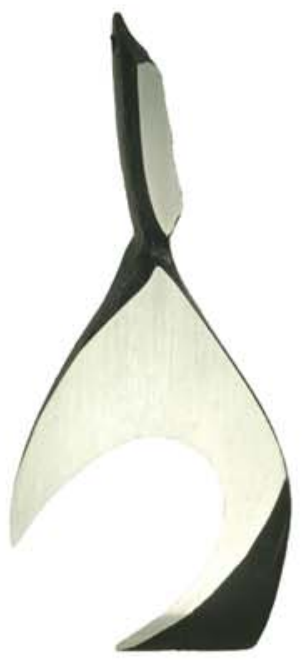

\# 146 


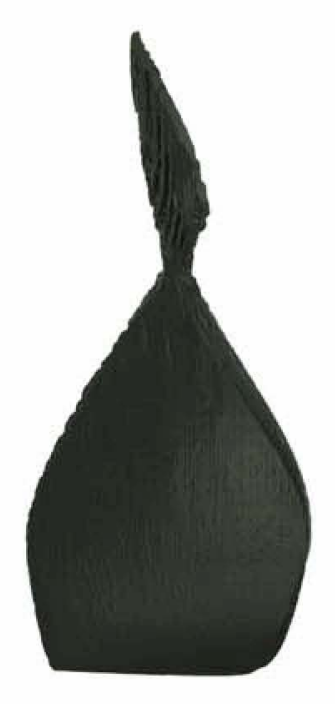

\#147

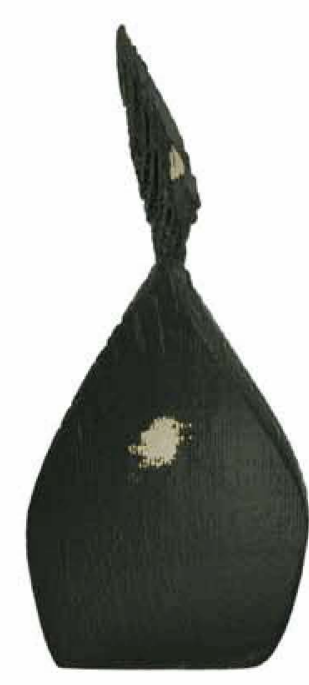

\# 148

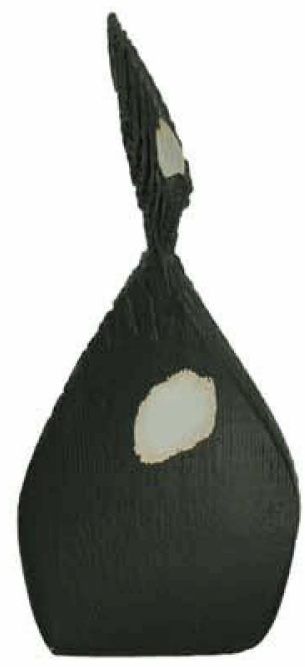

\# 149

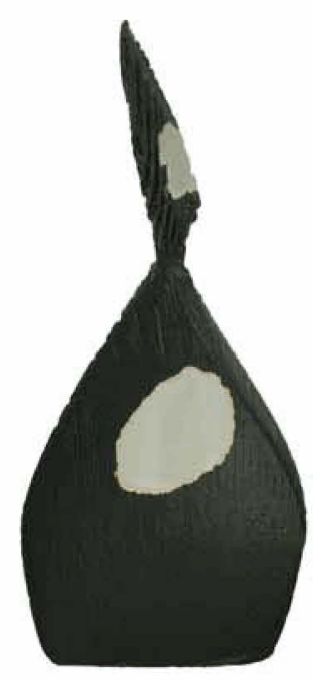

\#150

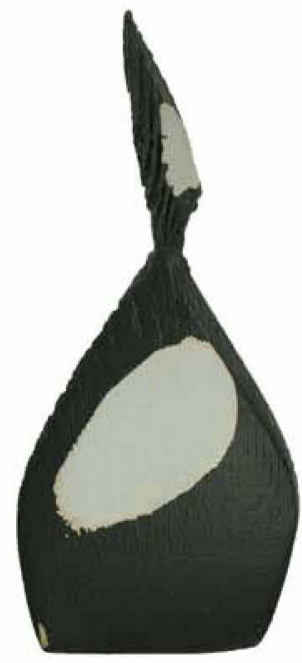

\# 151 


$$
\text { dbddd }
$$


Observations

The initial orientation of the product to the wear surface had a critical role in determining the eventual wear pattern. Products that balance on the surface relied on internal mass to define the initial orientation (Ref \# 78 \& 138). Once a wear surface formed the orientation became established and continued in a consistent manner.

Penetration through the products allowed the form on the underside to become visible and critical in the emerging wear pattern and overall shape of the product (ref sample \# 146). In the samples created using the FDM process, the fine surface texture on the underside of the product became revealed as a lattice (ref sample \# 134) that transitioned into a jagged edge (ref sample \# 135).

An acute angle between the product and the flat surface of the wear mechanism revealed initially unperceivable variations in the products form (ref sample \# 100). The contrast between the matt black paint finish and the underlying zinc casting highlighted these variations.

The defined 2D plane of the wear surface created patterns in the 3D samples similar to that of section cuts through the product

(ref samples \# 117-126). The wear patterns were primarily pre-determined and created by the form of the product and the wear mechanism

(2D surface). The wear patterns evidenced the frequency of use with limited information about the specific actions of the person.

The consistency of the interaction between the 2D wear mechanism and the 3D product could be foreseen (before the wear simulation) and could be simulated using existing computer programs. This defined interaction would make an ideal trial of $4 \mathrm{D}$ computer simulated wear patterns. 
5.14 Enclosure Wear (Pocket) 
Experiments: \# $157-236$

Materials: Oxidised copper (1 micron) / Copper electroplating (100 microns) / Zinc casting

Design Issue: The aesthetic qualities of wear that occurs in enclosed space that are intimately connected with the human body such as pockets in clothing.

A primary site for portable electronic products such as cell phones and music players in clothes are pockets. The wear action occurs as the products are taken in and out of the pocket, when the product is stored in the pocket and moved relative to the body. The flexibility and softness of the fabric used in the pocket combined with the dynamic nature of the site creates an environment offering a high rate of wear evenly across surfaces of the product.

To reduce the variables in the experiment the simulations were based on the pocket being empty of foreign objects that would scratch the surface such as keys and coins.

The exterior of the base zinc casting was electroplated with 100 microns of copper. The copper was then oxidised in a solution of

de-ionised water and liver of sulphur that turned the initially bright copper plating into a consistent matt black finish. A linen polishing wheel was used to simulate the low level of friction and high degree of movement likely in the site. This resulted in a finely polished surface. 


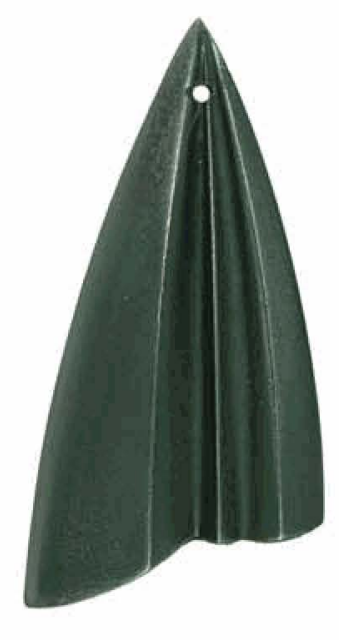

\# 197

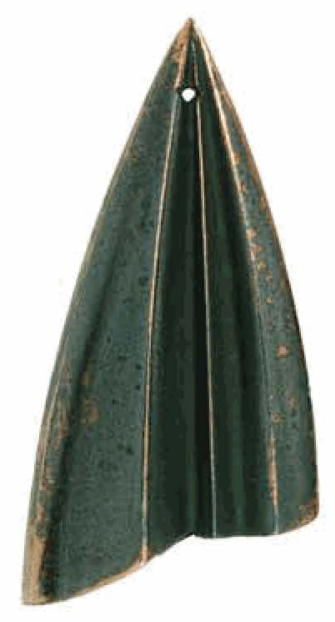

\#199

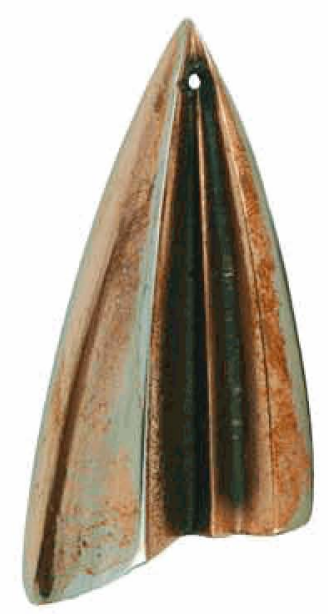

\# 201

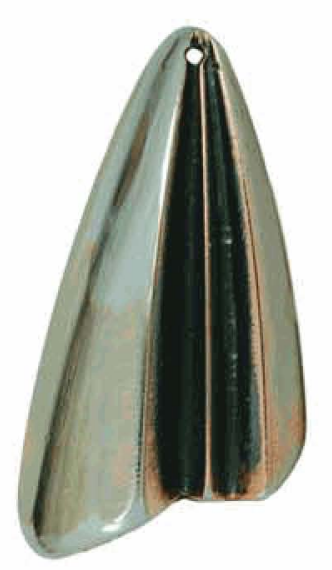

\# 203

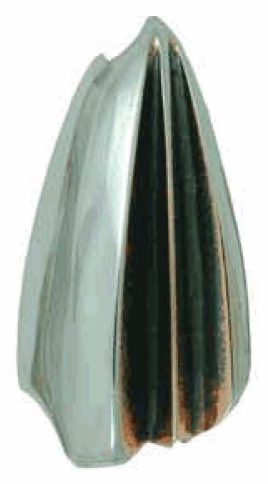

\#205 


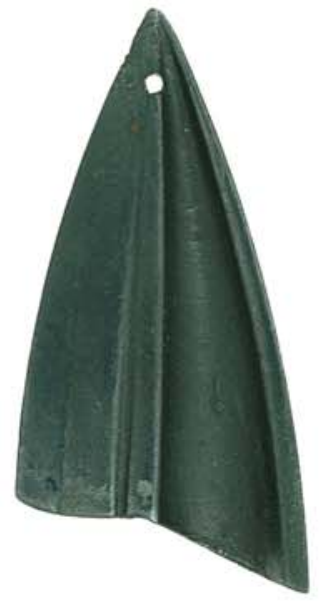

\# 198

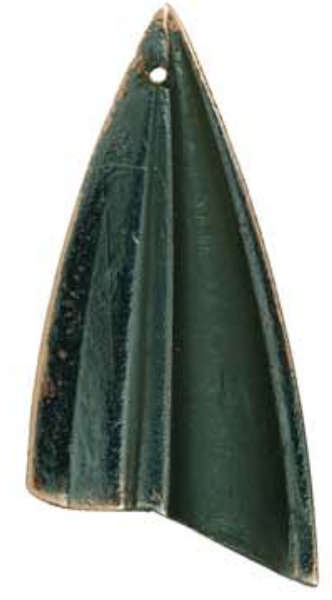

\# 200

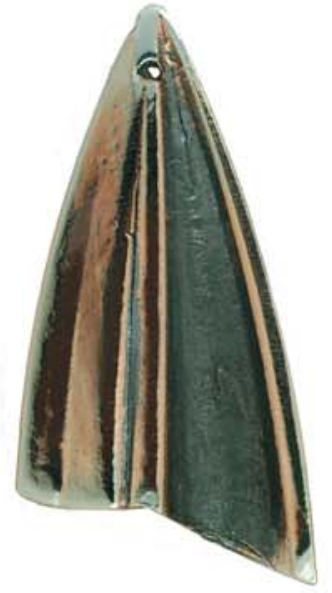

\# 202

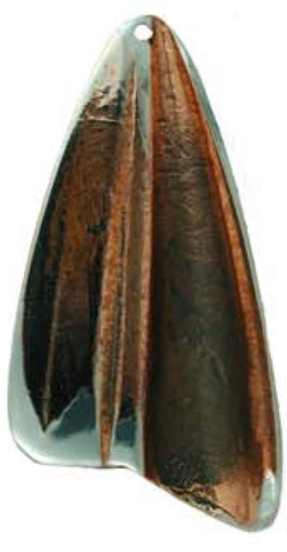

\# 204

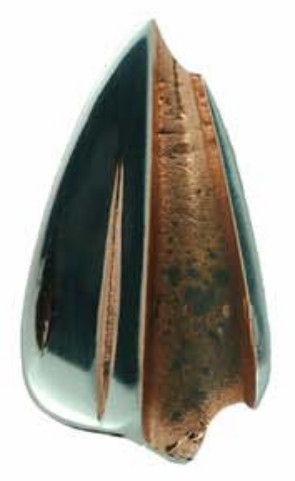

\#206 

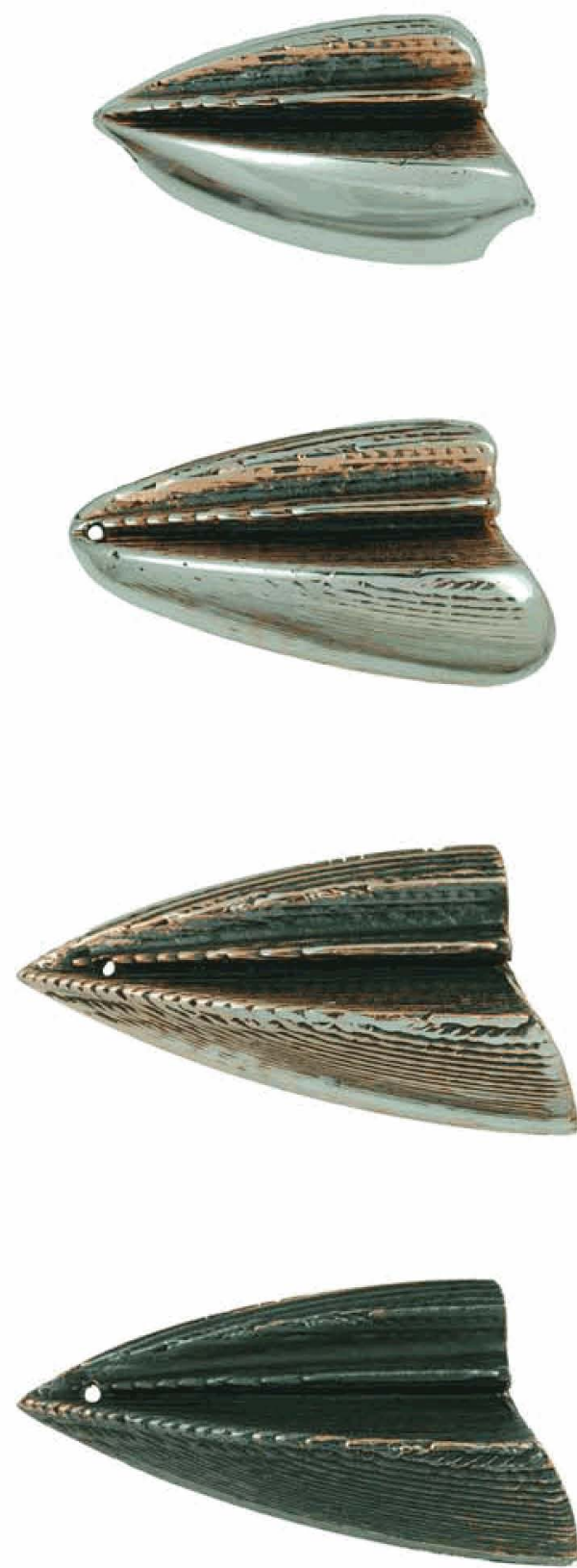

을

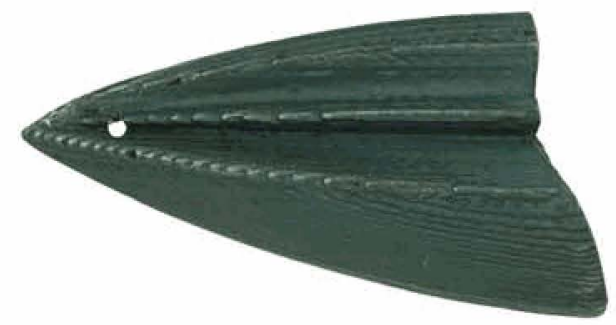

오 


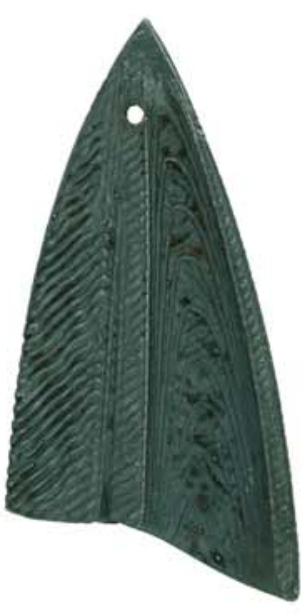

\# 208

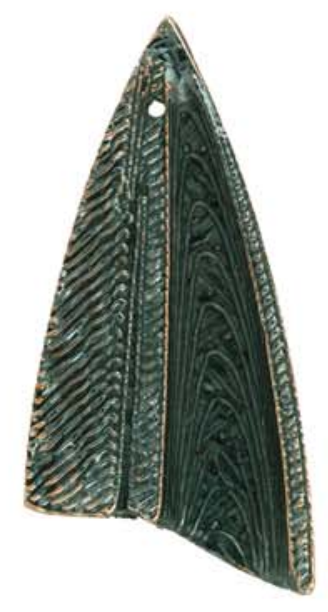

\# 210

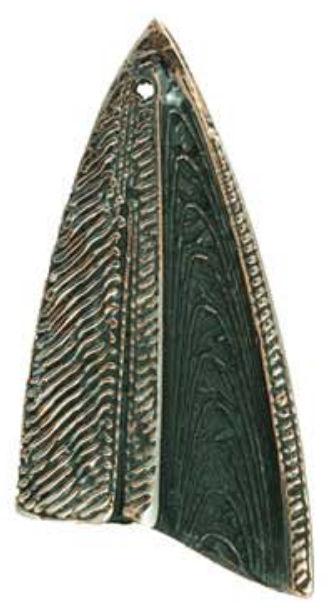

\# 212

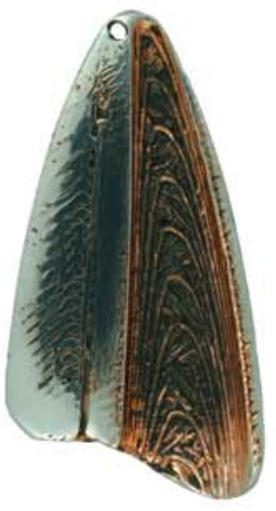

\# 214

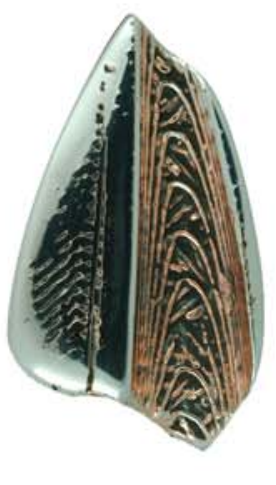

\# 216 

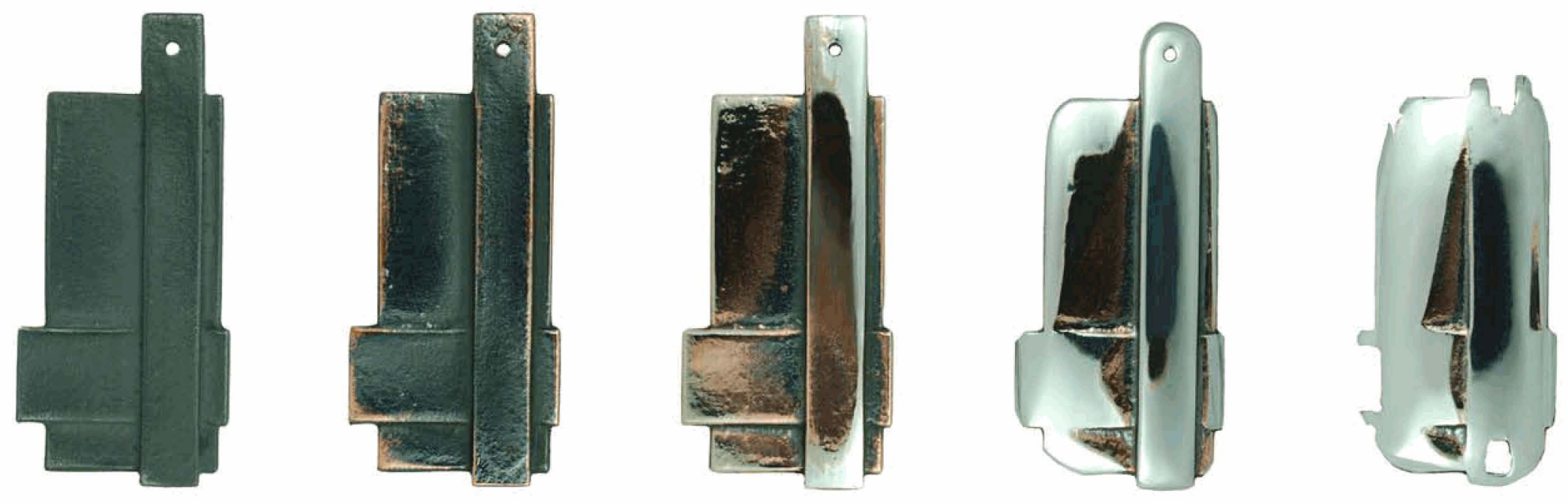

\# 157

\# 159

\# 161

\#163

\#165 

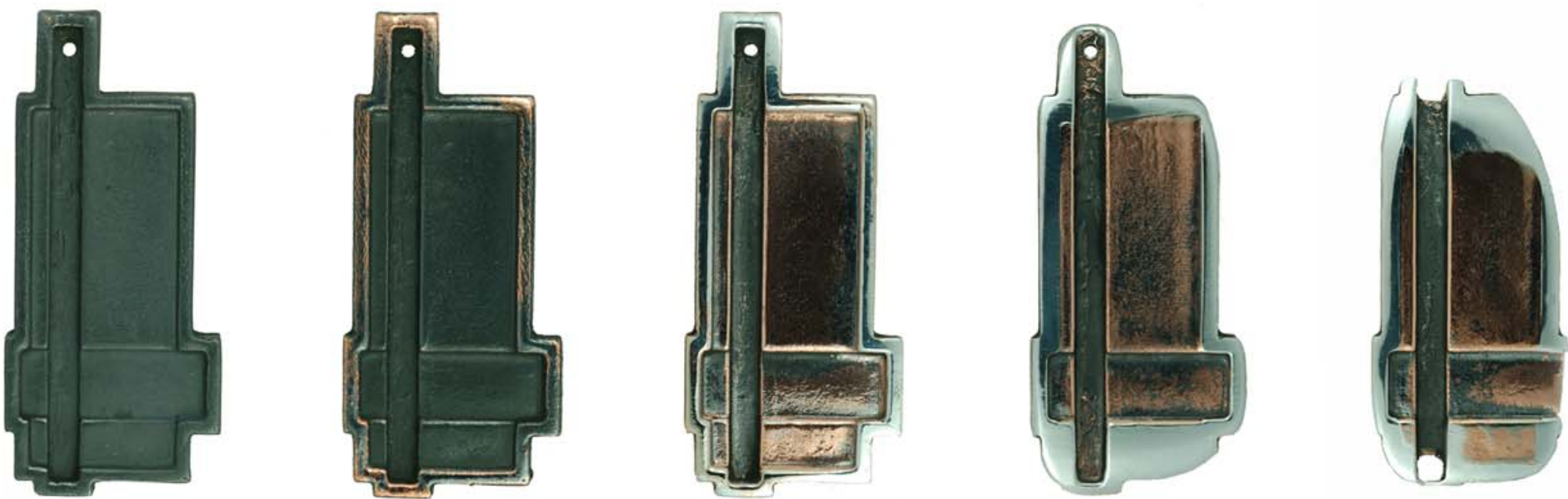

\# 158

\#160

\# 162

\# 164

\# 166 

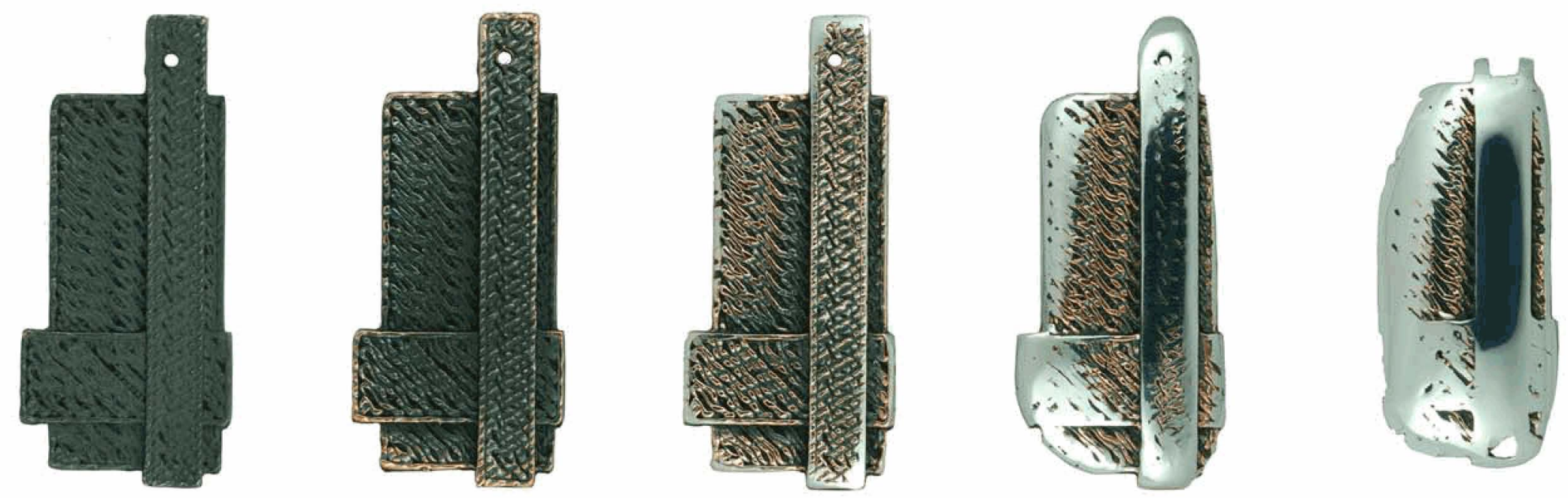

\# 167

\# 169

\#171

\# 173

\# 175 


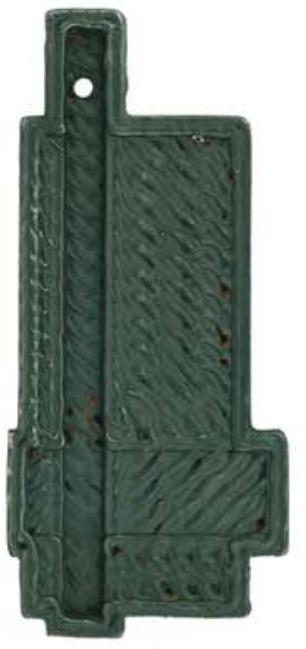

\# 168

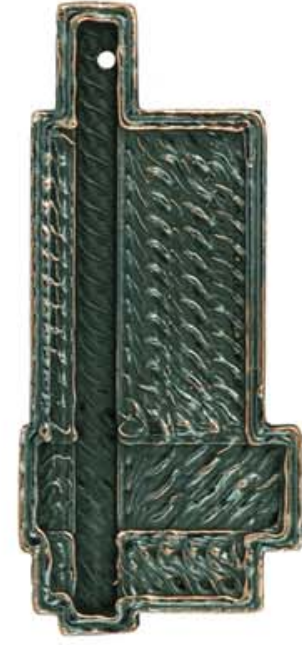

\# 170

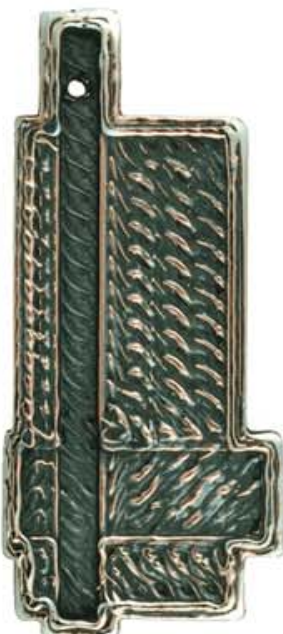

\# 172
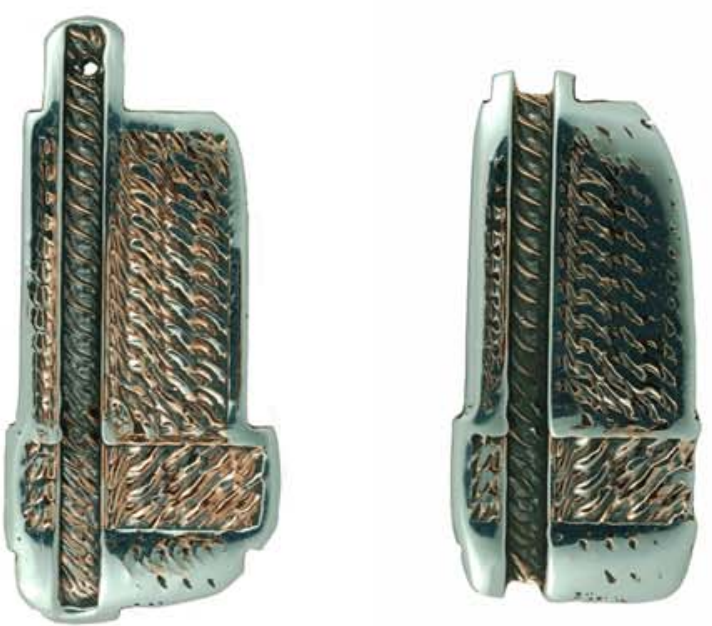

\# 176 

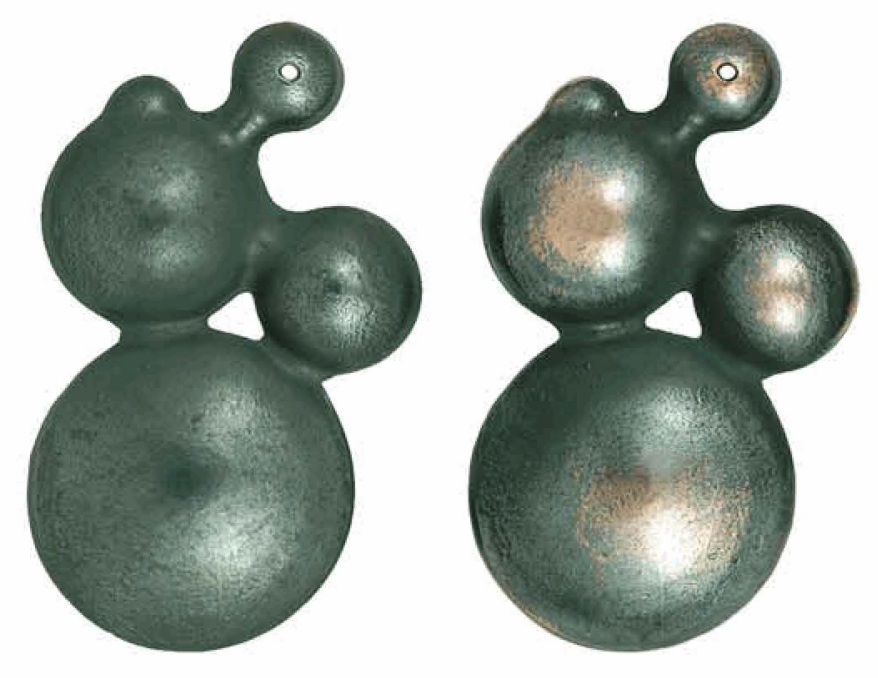

\# 177

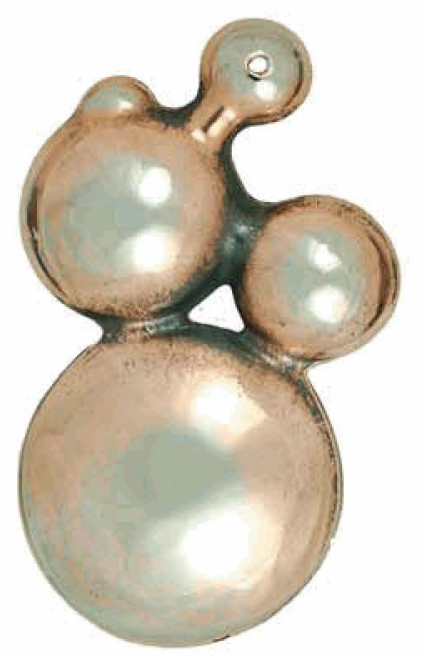

\# 181

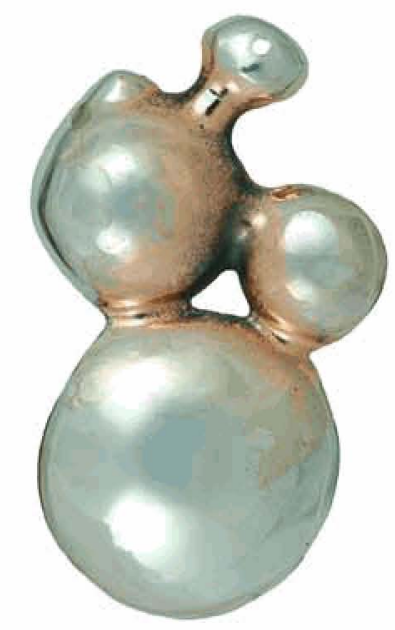

\#183

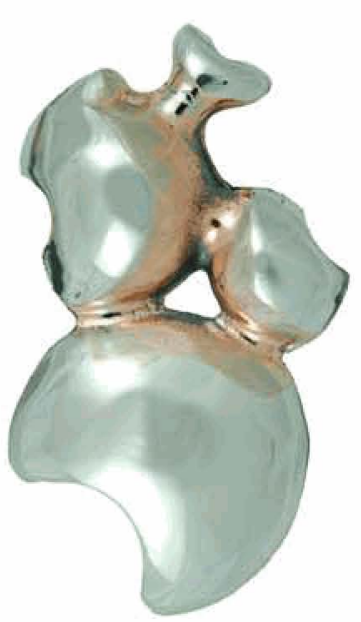

\# 185 

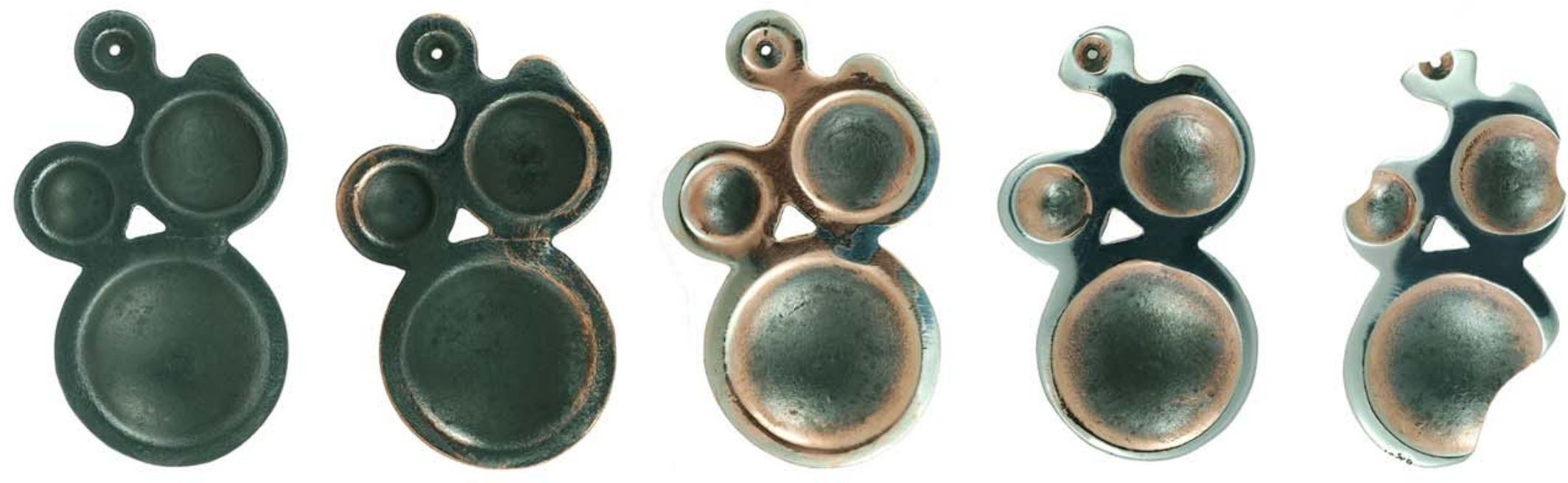

\# 178

\# 180

\# 182

\# 184

\# 186 


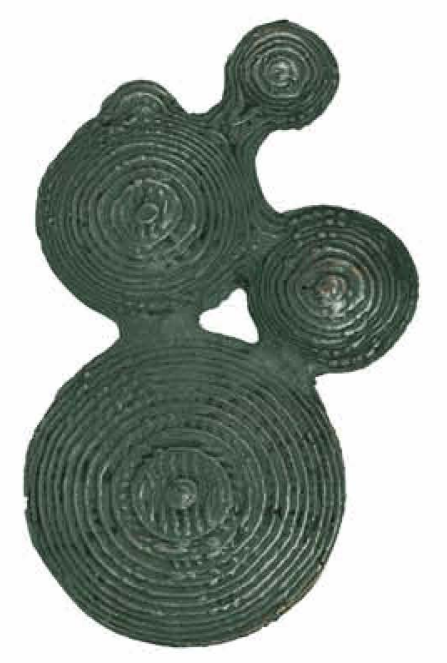

\# 187

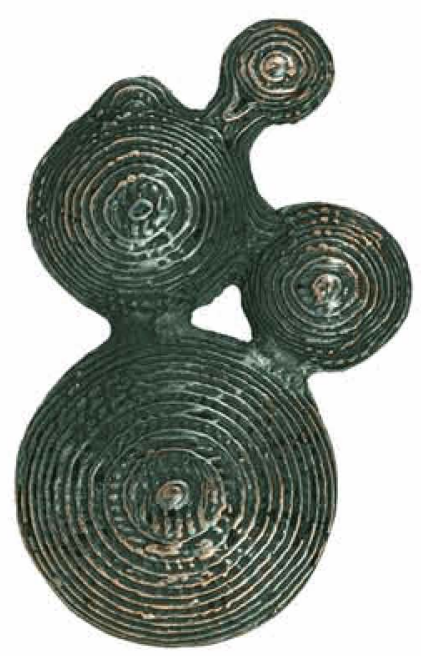

\# 189

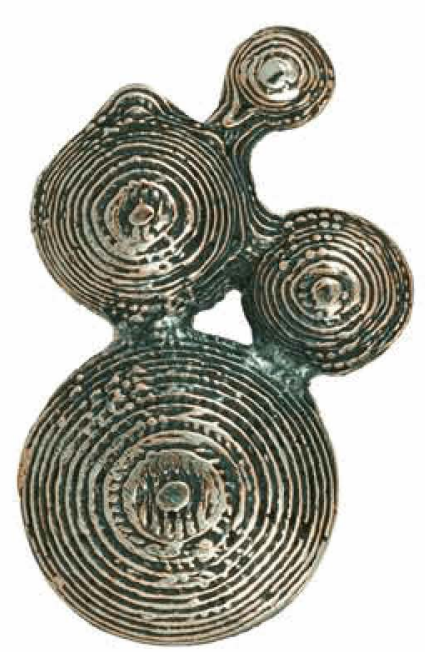

\# 191

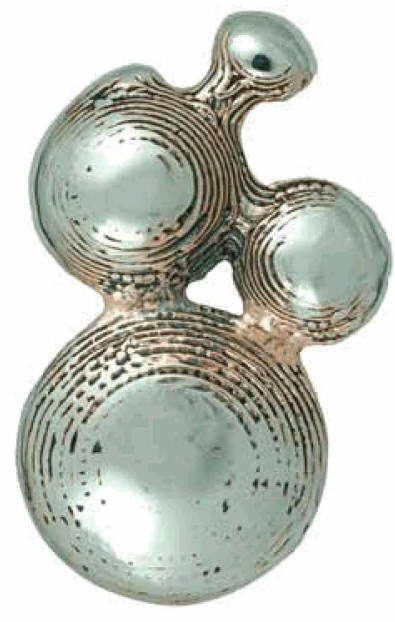

\# 193

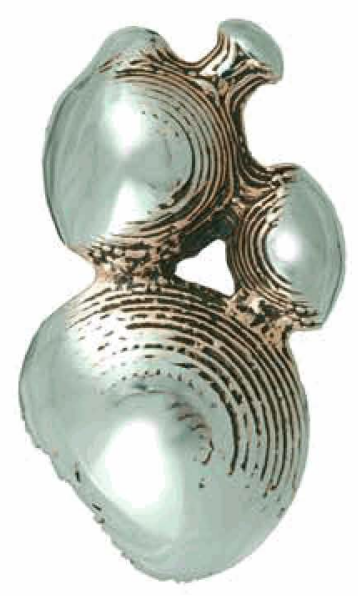

\#195 

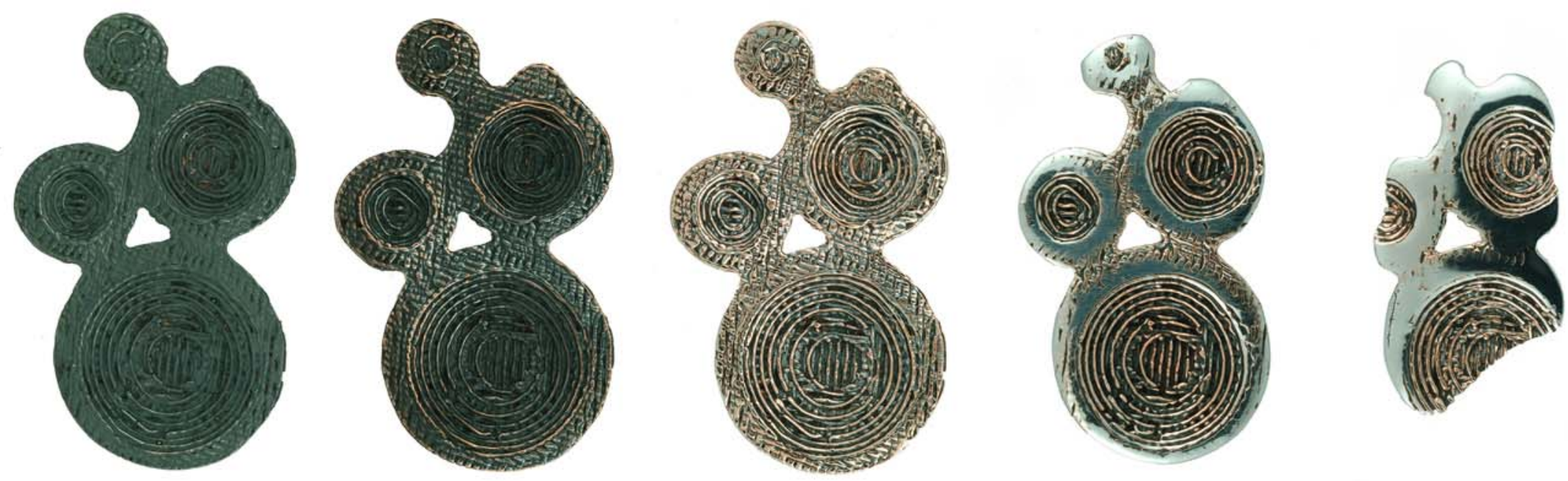

\# 188

\# 190

\#192

\# 194

\#196 


\section{Observations}

The flexibility of the fabric of the pocket created a wear mechanism that extended around the product creating a gradual transition between heavily and lightly worn areas. Protruding areas became heavily worn while recessed areas inacessable to the fabric remained unworn (ref sample \# 166). Uneven wear over the surface created a product in which new and old materials co-existed.

As the surface of the products wore away the pre-existing relationship between the copper oxide external layer and the underlying copper from which it was derived became evident (ref sample \# 179). This created a subtle transition between the two layers unlike that of a surface coated with a dissimilar material such as paint over metal. Once the copper layer was worn through, the zinc casting was revealed and polished.

The reactive nature of the copper plating contrasted the non-reactive zinc base material (ref sample \# 201). While the product was being used both materials produced a similarly highly polished surface. Yet once static the differences in reactivity became apparent as the copper continued to oxidise becoming dull while the zinc remained highly polished.

As the wear process evolved the transformation of the product's surface, it extended into a transformation of the product's form (ref samples \# 157-165). Once the wear had penetrated through the product the thickness and form of the other side started to dictate the product's overall form (ref samples \# 185 \& 186).

The fine ridges and hollows generated through the fuse deposition process (FDM) extended the plating further into the product's surface to create a layer between surface and form. As the top of the ridges became worn, the contrast between the layers highlighted the original subtle lines left from the construction process (ref sample \# 161 \& 171). Continual wear eventually removed the texture and revealed the samples as casting without the internal complexity of the original. 
5.15 Applied Design 
Experiments: \# $243-272$

Design Issue: Synthesising the information generated through the earlier pure design experiments into applied design products.

A series of products were produced (derived from the "Apple Shuffle") to allow a contextual and holistic assessment of the principles developed in the earlier experiments. The "Shuffle" was selected for its simple geometric form that allowed the application of multiple graphic layouts to be applied to the front and back surfaces. It could also be easily manufactured, was small in size ( $90 \mathrm{~mm} \times 25 \mathrm{~mm} \times 7 \mathrm{~mm})$ and was subjected to a high

On the rear of each sample a series of icons were used (similar to hallmarks) that revealed non-evident information about the product to include the company, the design team that produced the product, and the material layers beneath the exterior layer. By revealing these layers in the new product a potential customer could imagine how the product would change before it occurred, allowing informed consumption. The wear in the products was simulated using an abrasive paste that mimicked the actions of the finger manipulating the product.

Two areas on the back of the product where designed to be left blank by the factory to allow the retailer and purchaser to enter unique information such as the date of purchase and the fingerprint of the new owner. Ultra violet cured paste would be used in these areas that would cure when exposed to sun light (ref chapter 3 for a detailed explanation). 
Experiments: \# $243-248$

Brass (1 micron) / Copper (200 microns) / Translucent Red Plastic (Acrylic) 

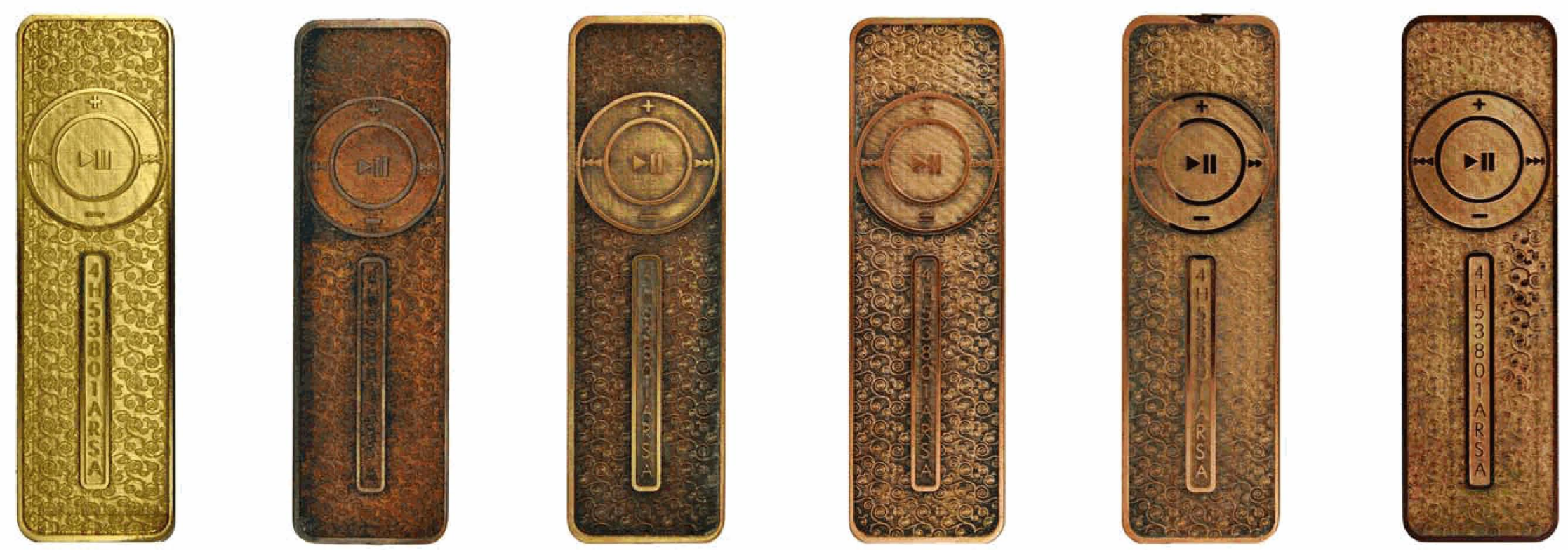

\# 243

\# 244

\# 245

\# 246

\# 247

\# 248 

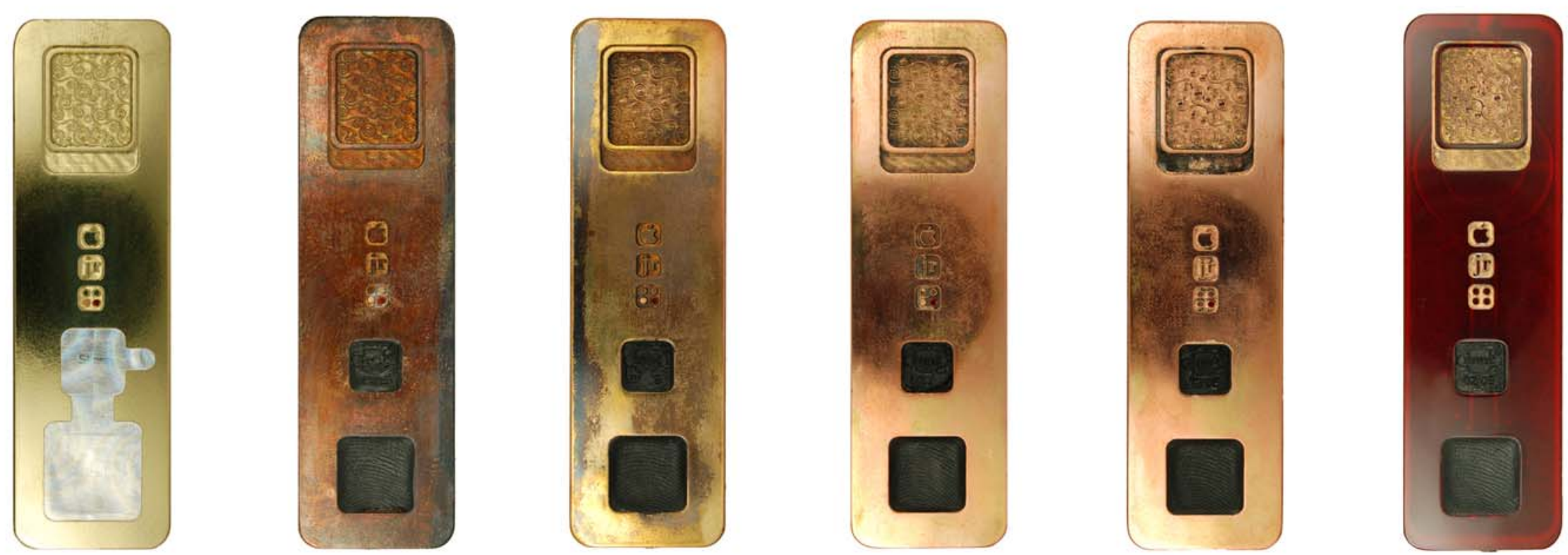

\# 243 
Experiments: \# $249-254$

Satin Nickel (15 micron) / Copper (200 microns) / Opaque Orange Plastic (Acrylic) 


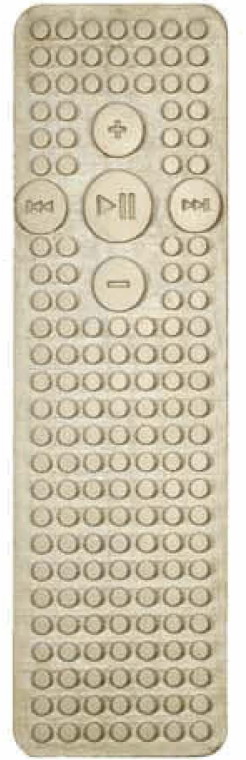

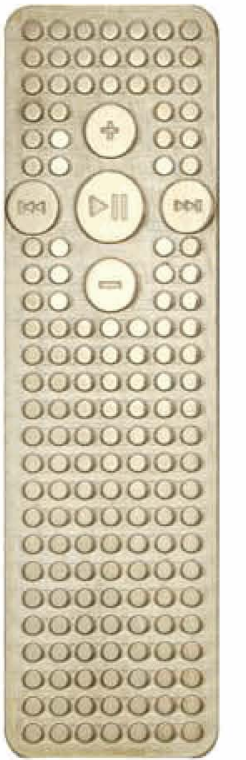

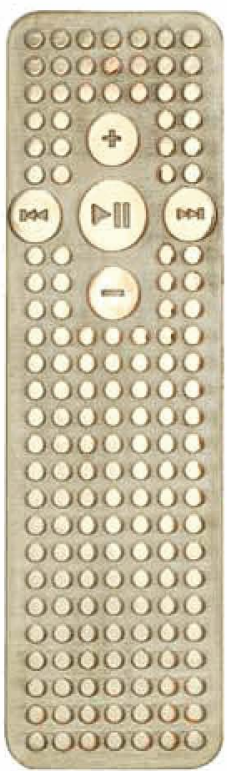

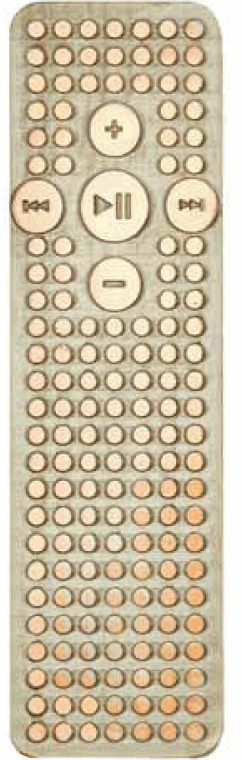
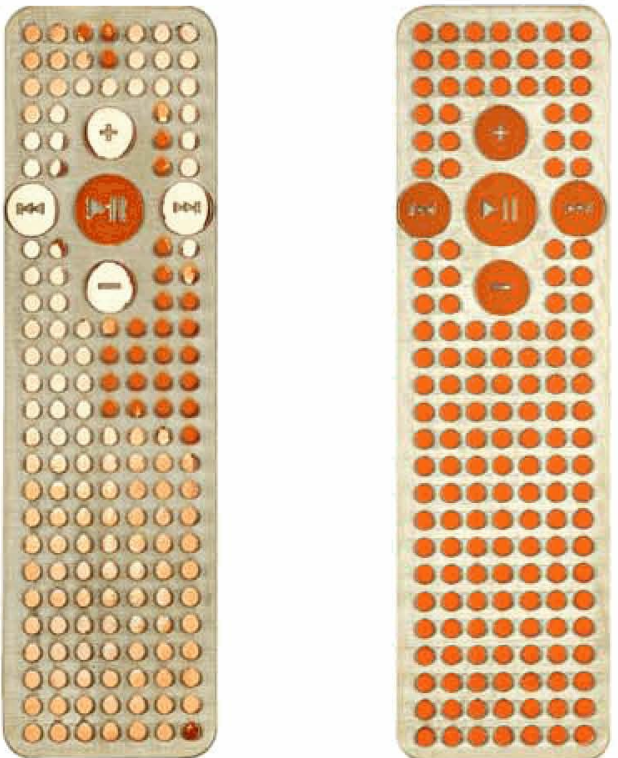

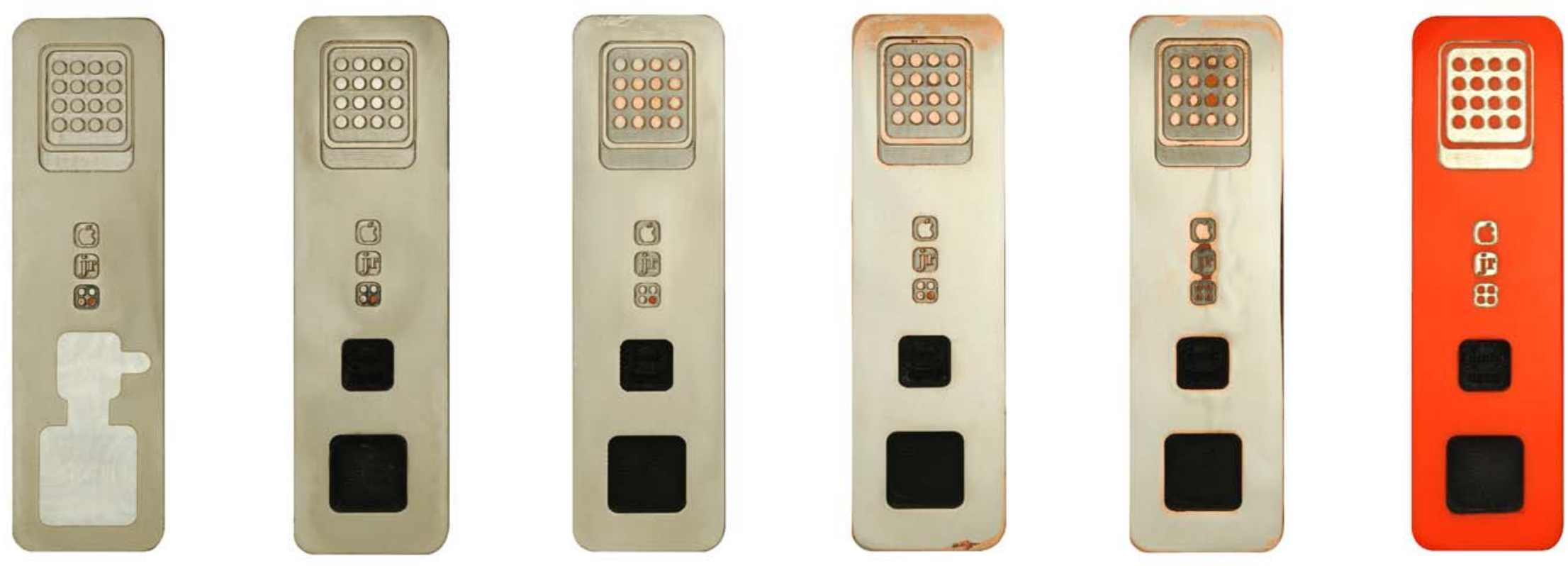

\# 249

\# 250

\# 251

\# 252

\# 253

\#254 
Experiments: \# $255-260$

Silver (1 micron) / Copper (200 microns) / Translucent Neon Green Plastic (Acrylic) 

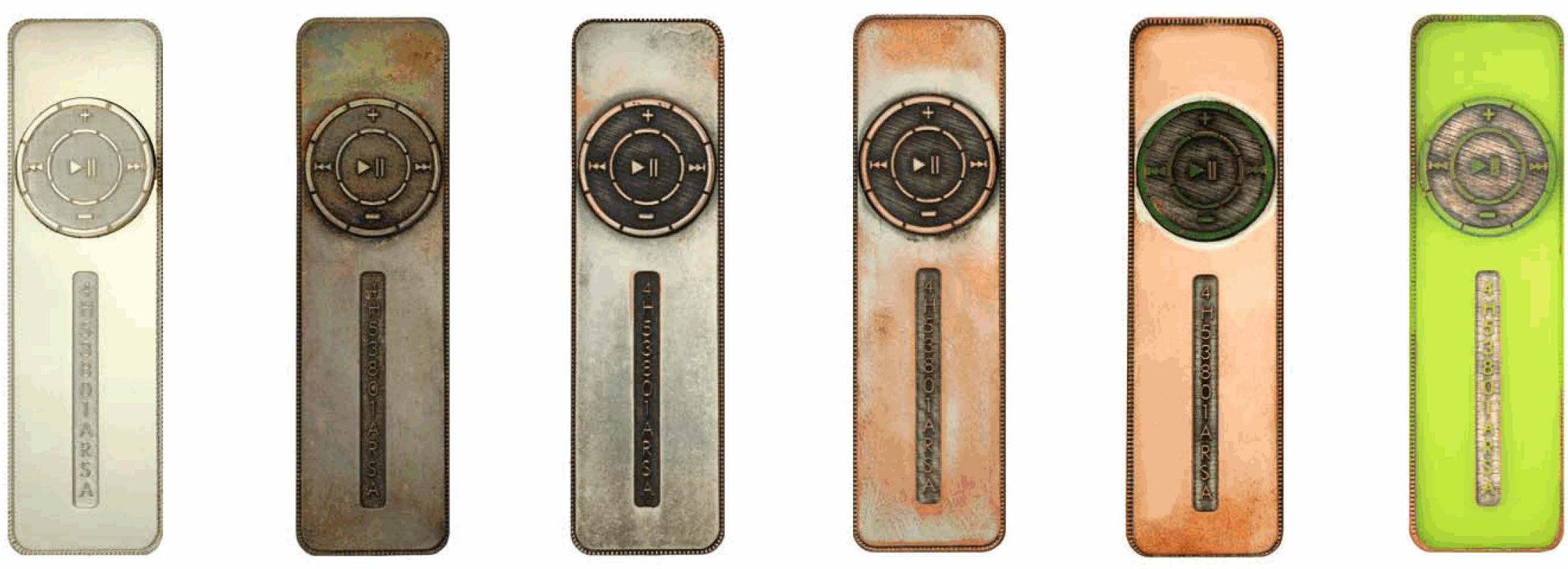

\# 255 

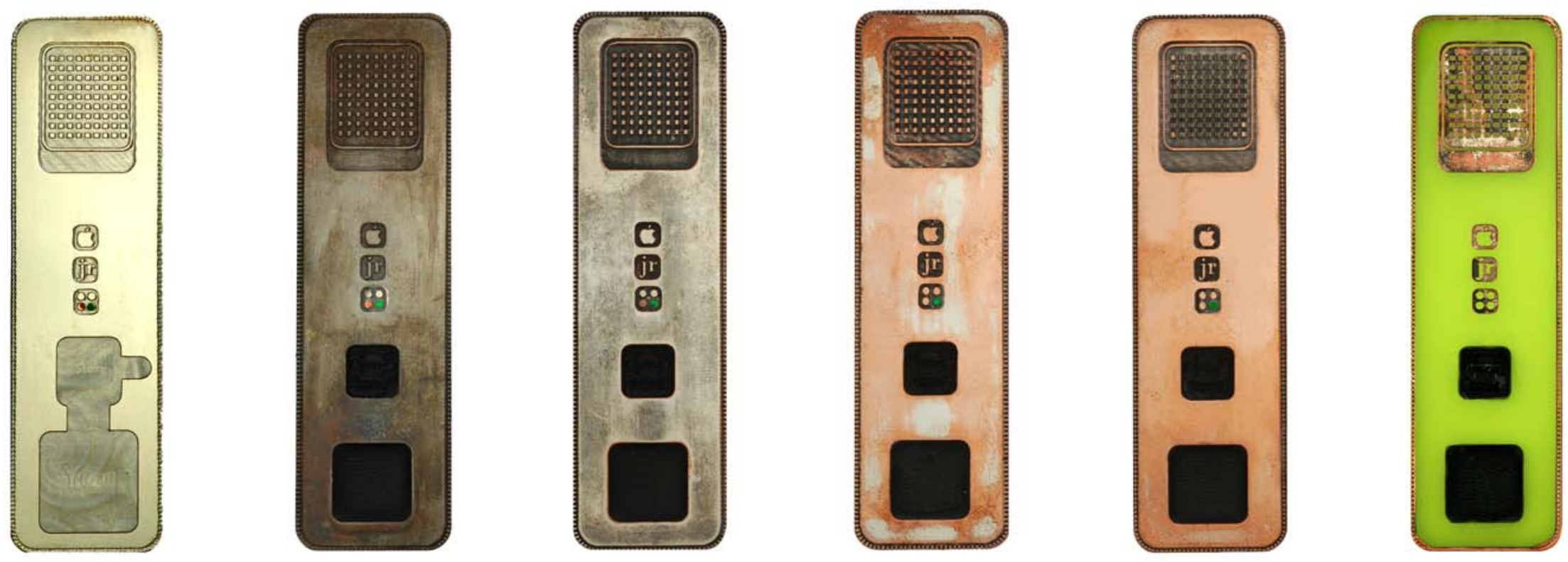

\#255

\# 256

\# 257

\# 258

\# 259

\# 260 
Experiments: \# $261-266$

Yellow Passivated Zinc (15 micron) / Copper (200 microns) / Translucent Orange Plastic (acrylic 

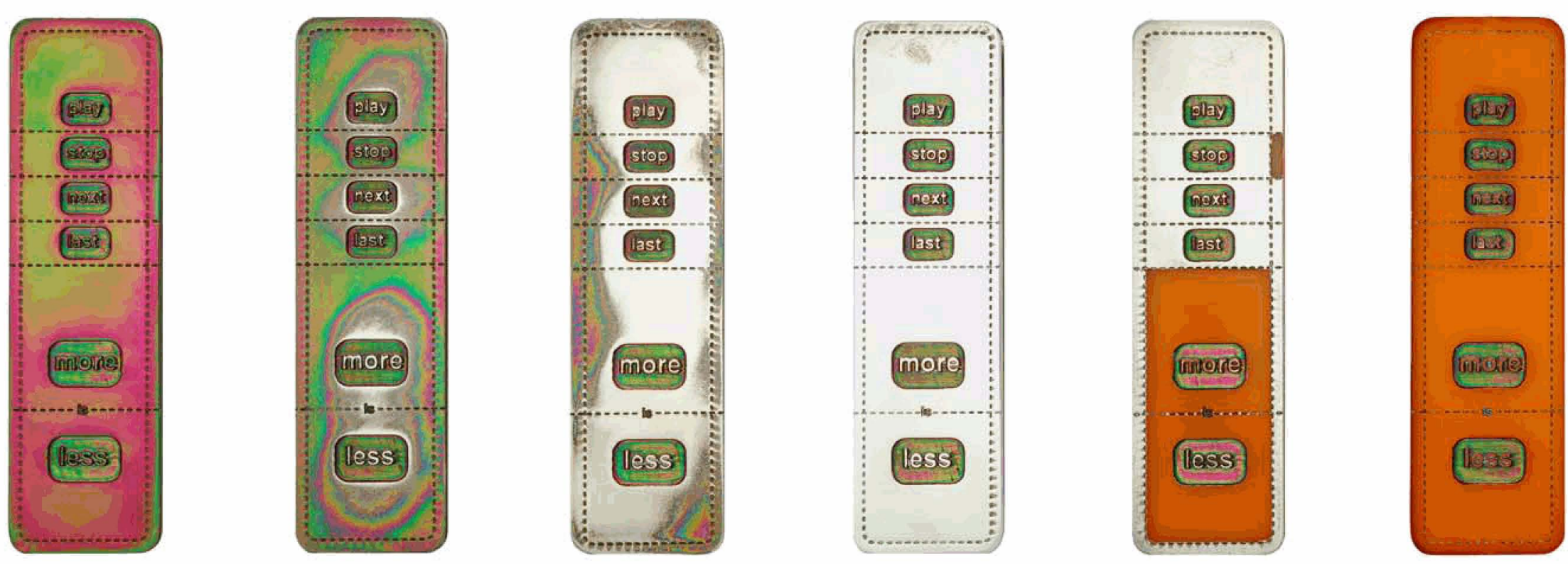

\# 261

\# 262

\# 263

\# 264

\# 265

\# 266 

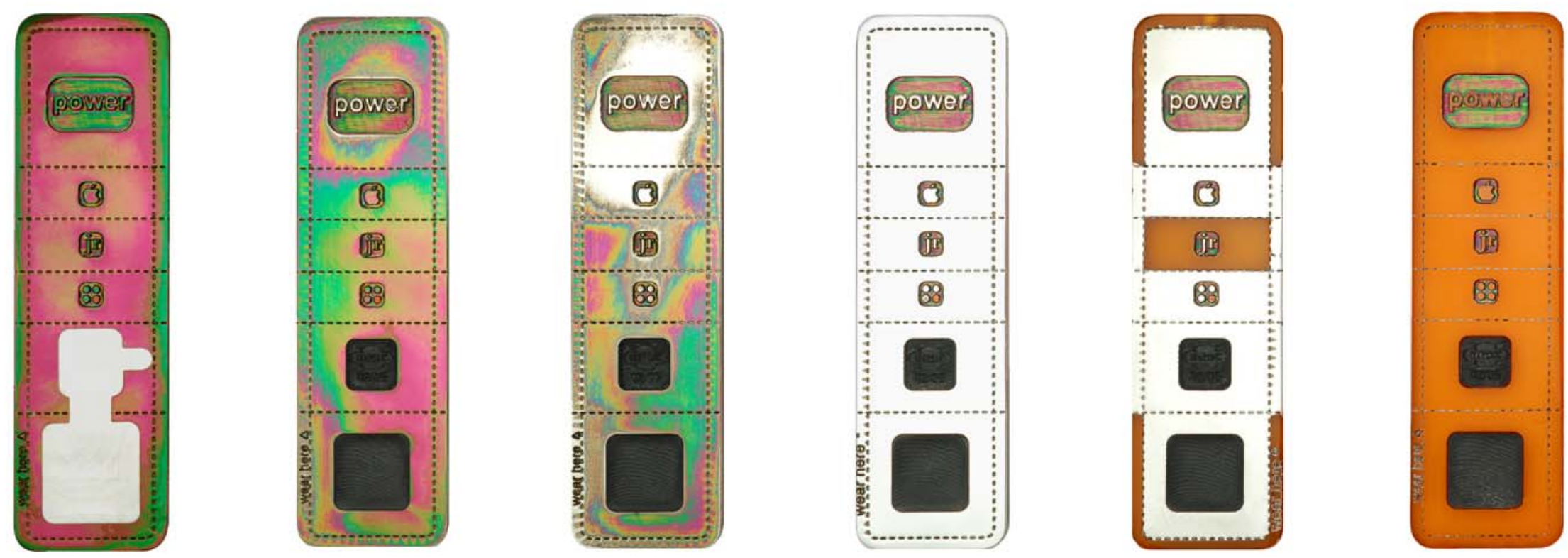

\# 261

\# 262

\# 263

\# 264

\# 265

\# 266 
Experiments: \# $261-266$

Yellow Passivated Zinc (15 micron) / Copper (200 microns) / Translucent Orange Plastic (acrylic 

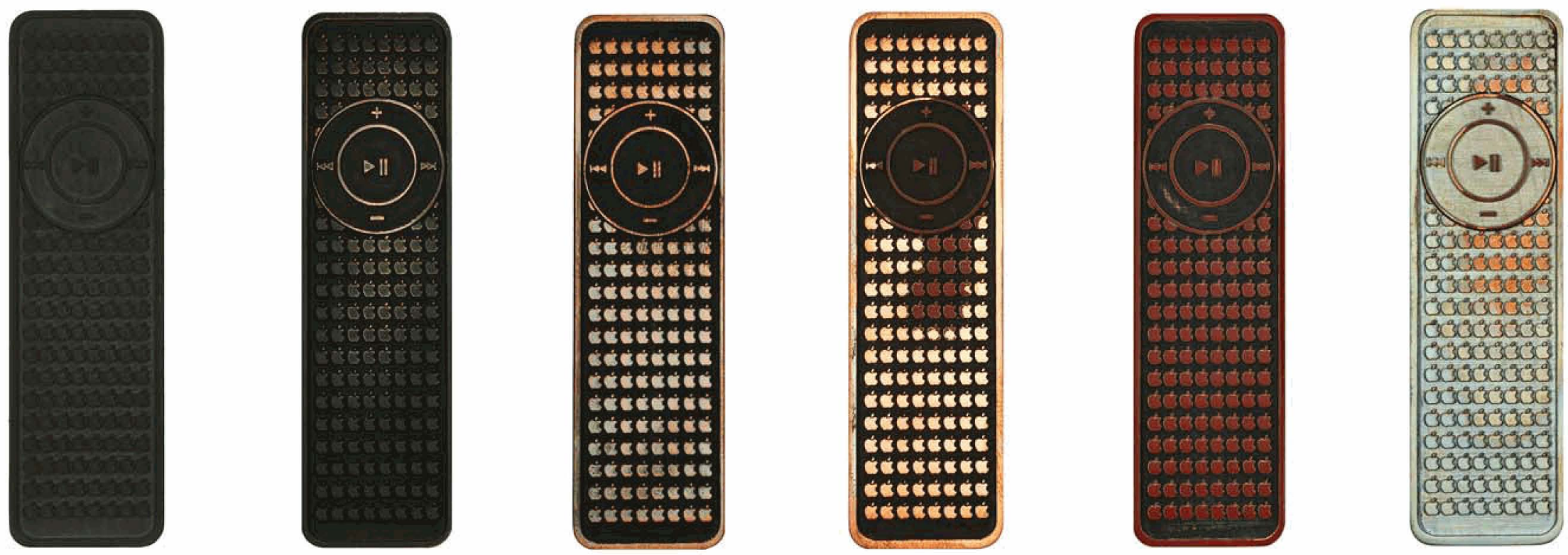

\# 267

\# 268

\# 269

\# 270

\# 271

\# 272 

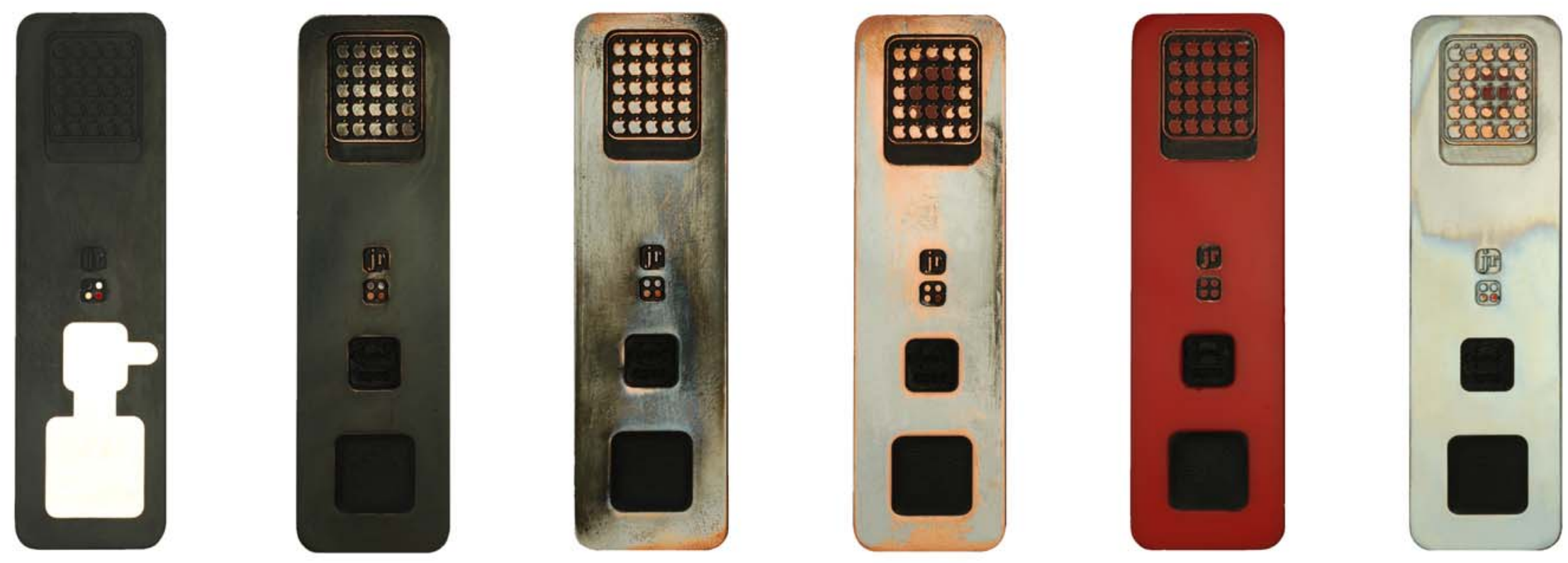

\# 267

\# 268

\# 269

\# 270

\# 271

\#272 


\section{Observations}

Metallic electroplating over the base plastic created a range of finishes of higher precision and material diversity than the initial painted samples. Reactive metals revealed the potential to create ongoing transformations in the product beyond its initial

manufacture.

The original bright brass and silver plating was oxidised in a water and sulphur solution to create a darker matt finish. As the product wore the oxidisation layer was removed allowing the polished metal to be revealed, creating a perception of improvement through use (ref samples \#

244-245). Retarding the initial finish quality with an oxidisation layer meant that the visual qualities of the product would be reduced when sold which could have detrimental commercial implications.

The passivation process (ref samples 261-266) of submerging the zinc plated products in a solution of deionized water and liver of sulphur created a reaction that formed a highly reflective spectrum of colours over the surface of the product (similar to a CD). Unlike the dull

oxidisation on the brass and silver samples the glossy passivation appeared desirable when new. As the passivation layer wore through the colours became more intense around the areas touched (ref sample \# 262) until consistent zinc below was reached (ref sample \# 264).

This process offered highly visible transition as the product became worn without the need to retard the visual qualities at any stage.

The visual transitions that occurred on the non reactive metal were more subtle yet still perceivable. The non-reactive qualities of nickel were utilised to create a product that remained "clean" as it wore. The satin finish on the nickel eroded to produce a polished surface that subtly revealed use patterns (ref sample \# 250). By focusing the wear energy onto a smaller area ( $2.5 \mathrm{~mm}$ circles) the variability of the wear pattern was visually controlled and contained. The use of bright colours below the metallic plating allowed a dramatic and deliberate contrast between the layers with an abrupt transition between them as sections of the metallic plating delaminated.

In an attempt to acknowledge and harness the delaminating quality of the electroplating a series of raised perforated lines were engraved into two products (ref samples \# 261 \& 255) as weak points similar to the perforated lines used in cardboard that allow defined tear paths . Once the edges wore through large sections of the plating pealed away revealing the bright colour below (ref samples \# 260 \& 265). The delamination of the plating from the base plastic was not initially desired (ref sample \# 253). When considered as a transition, distinct from traditional slow erosion, it emerged as a valuable wear process capable of creating more abrupt change.

The requirement to consider the visual balance of a product throughout its life became apparent with the black zinc product (ref sample \# 267). As it eroded through to the underlying zinc the fine detail became evident in a user specific pattern (ref sample \# 268). The use of the repeated manufacture's brand to create the focused wear areas (Apple logo) produced a product that became visually more intensely branded during use: A preferred transition would reduce the prominence of the brand as the product became more intimate and related to the user. The order of the transition needed to be re-considered during the initial design process.

The flexibility of computer controlled processes used to make the samples allowed large individual product serial numbers to be incorporated into the front of the brass and silver products (ref sample \# $243 \& 255$ ). As the oxidisation wore through this detail became more legible. 

6. Conclusion 
Arguably an underlying tenet of the Industrial Design profession is to create products with maximum visual appeal when they are new. Implying a designer's engagement and responsibility ceases once the product is sold. Through this research new ideas and strategies have emerged which

extend the designer's vision and control of the product beyond inception into its life to be transformed through their interaction with people.

The capacity of designers to engage and to comprehend the implications of their actions extends their responsibility to know more about the future of their creations. With increasing public awareness of the accumulating ecological, social and cultural effects of mass consumption, a need is emerging to rethink the role, focus and methods of the Industrial Design profession.

The responsibility for the creation of products and their ensuing effects is equally shared by academics with a role in determining a critical perspective. In the architectural profession the relationship between theory and practice has a much longer established history and range of research methodologies. An interplay between the practice of architecture and the theories related to architecture has offered critical insights that inform and evolve each others perspective. Industrial Design with a younger, less defined and developed relationship between theory and practice has a limited discourse which inevitably has impacted upon the development of the discipline.

This Design Research by Composition proposes an approach that mediates between theory and practice by transposing the traditional physical modelling process (used to create products) into a research method to explore issues related to products. The information and knowledge gained through this research approach is inherently physical and visual and thus evidently relevant and transferable into design practice. The ability to materialise theoretical issues through physical design experiments has allowed a rigorous method to evolve; one that is capable of creating a reaction between people and products beyond the imaginary and thus purely, theoretical discovery. The development of research methods to understand complex interactions and their implications will allow the Industrial Design profession to mature and establish a critical role in evolving the philosophies underlying consumerism into a contemporary, sustainable and visionary philosophy of post-consumerism.

The ongoing cycle in the process of observation and experimentation has evolved into a design research method which has become the foundation of this research. The initial tightly defined pure design experiments that focused on specific issues evolved into applied design experiments which synthesised the information into tangible products. The sequence and evolution of the experiments illustrates the growth of subject specific knowledge. As a primarily visual research method and resource, all results of the Worn Out or Worn In? experiments have been catalogued and displayed to substantiate the process and outcomes. 
The interpretation of the results from the design experiments and the speculation regarding their broader implications have evolved into a process termed design fiction; borrowed from science fiction but grounded in the material sensitivities of Industrial Design. As the speed of technological change increases, so does the need to look into the future to foresee the implications of the emerging technologies. The ongoing development of computer technology offers a potential tool to access the 4D design realm. The use of physics based programs capable of calculating material qualities could allow the interaction between people and products to be accurately, visually and quickly simulated to offer a lucid vision of the product's complete life cycle.

Comprehension throughout a product's life would enable Industrial Designers to work with the dimension of time to create products with a defined life expectancy; from disposable products with a clear vision of their construction, use and de-construction to enduring products with a designed transformation from generic commodities to personal artifacts. An understanding of time not as a singular instant but as a continuum allows multiple design opportunities to exist within one product. This extends the scope of design beyond a narrow definition of physical function into a personal function that connects people to their possessions and ultimately records the history of a person in their possessions. Evidence of a human relationship embedded in a product can transform it into a meaningful artifact, an heirloom capable of connecting generations. This understanding of products as possessions, artifacts and heirlooms acknowledges their role as collectors and connectors between people as individuals and people as socially and culturally interrelated groups.

As the Industrial Age evolves into the Information Age, a challenge is emerging for Industrial Design to maintain its relevance by transferring allegiance to the emerging field of Information Design. This realignment shifts the focus from the manufacture of products into the design of interactive and informed relationships between people and products. The designer's role of foreseeing, choreographing and disseminating information offers the creation of functional, evident and symbiotic relationships. The increase in knowledge about physical and digital qualities offers a celebration of change and life manifested through 4D designs as they become Worn Out or Worn In? 

Appendix: Site Observations 



\section{Site Observations}

This research started by observing actions and reactions that occur through time in an attempt to understand the ongoing relationships between people and products.

The lack of a specific site for industrial designers to exist within and create from is arguably why mass produced products are often lacking in

qualities that emerge for context. The human body is considered generic, measured and categorised into ergonomic percentiles or market demographics. This averaging of information denies the subtlety and diversity of interaction between people and products and the ways these become recorded in the site. The site in this understanding is not the product itself or the person, rather the 'space' between the product and person. The site is formed through the evidence of occupation.

The following images taken at the formation of this research explore evidence of occupation between people and products. Beyond the physical evidence, they offer a glimpse into the emotive qualities evolved from the intimate and personal relationship that exist between people and the products they have made through construction and re-made through use. 
arose from the focus on choosing a silverware pattern whose supposedly functional ends followed in line with their fashionable handles rather than adhering to the classic designs that had evolved in response to the failure of their predecessors effectively to separate food on the plate and convey it to the mouth.

Whatever the shape of its tines, making a fork out of a more common metal, such as britannia (a compound of tin, copper, and antimony that surpassed pewter in luster and hardness), and plating it with a fixed amount of silver produced an affordable piece of flatware. If thicker tines and more elaborate handles added surface area to be plated, then the plating could be thinner. The often heavily decorated handles that distinguish one silverplate pattern from many others were clearly the focus of illustrations in mailorder catalogues. Frequently the identical bowls of spoons were overlapped as the handles fanned out in a display of choices in the same price category. Elsewhere, handles only were pictured in an arrangement of patterns radiating out from a text announcing "quality" and "charm" and assuring the shopper that "no effort has been spared in making this service correct, individual and charming." Individuality seems to have been an important selling point,

with engraving offered either free or at a nominal charge, and the guarantees of at most a lifetime suggested that the silver was not intended to be bought for one's descendants but to express one's own individuality.

In the wake of the government-sponsored simplification system, there appears in such catalogues also to be a diminishing emphasis on specialized pieces of silver, such as oyster forks and fish knives, and a growing emphasis on serving pieces, such as sugar shells and gravy ladles. This was in keeping with a return to dining en famille after the late-nineteenth-century vogue of diner à la Russe, in which the entire dinner was served in the Russian fashion from a side table, with no serving dishes appearing before the diners. Today, the dexterous use by waiters of the large fork and spoon in concert, rather than the employment of specialized serving pieces, reminds us of how versatile a few pieces of silver can be in practiced hands.

Even in catalogues of some of the best modern silver, handles are much less likely than blades or bowls or tines to be obscured or omitted from an illustration. In an exhaustive catalogue of silverplate patterns intended for collectors, nothing but handles are 
The lifetime guarantee was introduced to limit consumers' expectation to pass the product between generations. 
Products were marked with a full description of the maker and materials used.

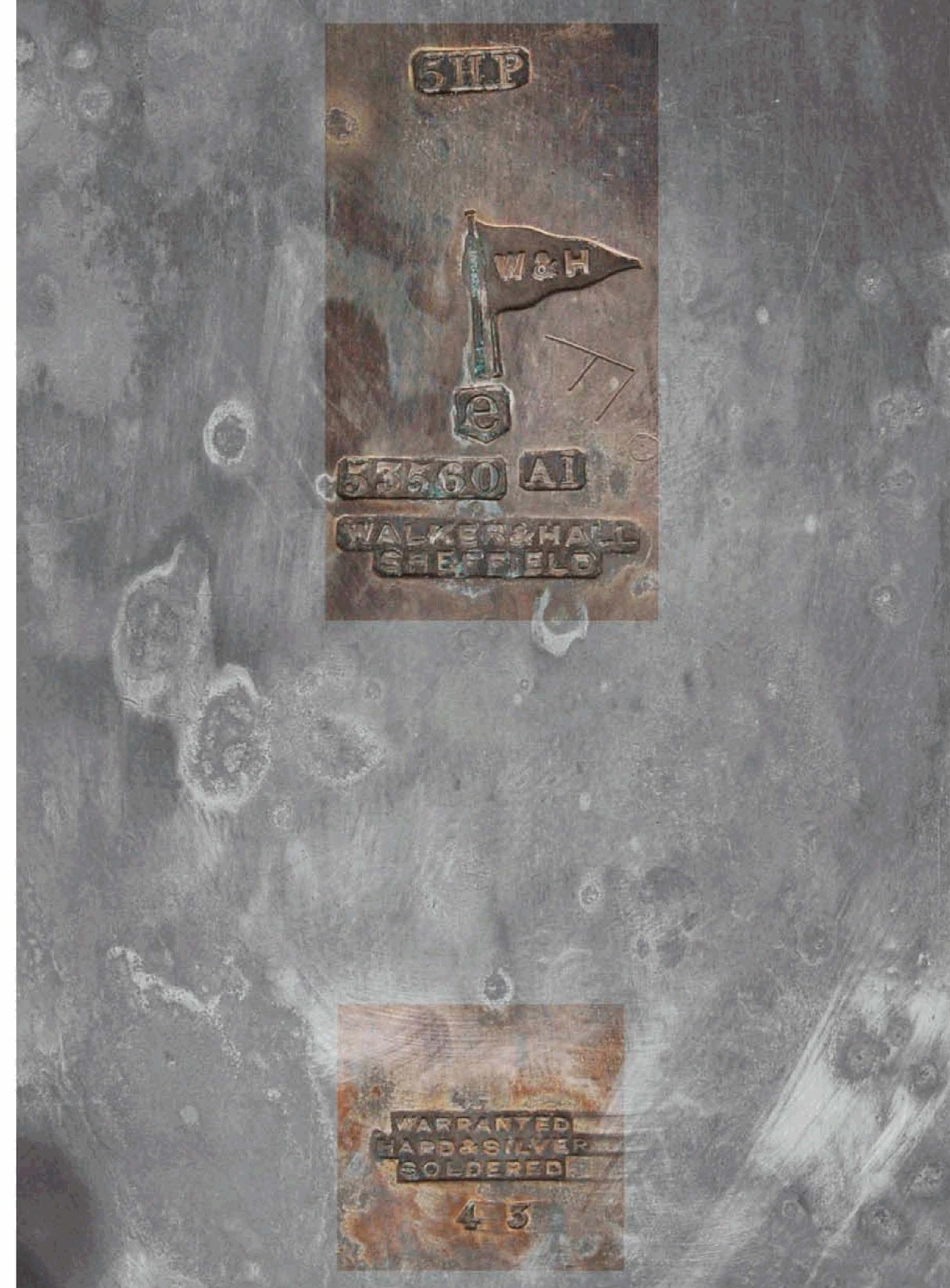



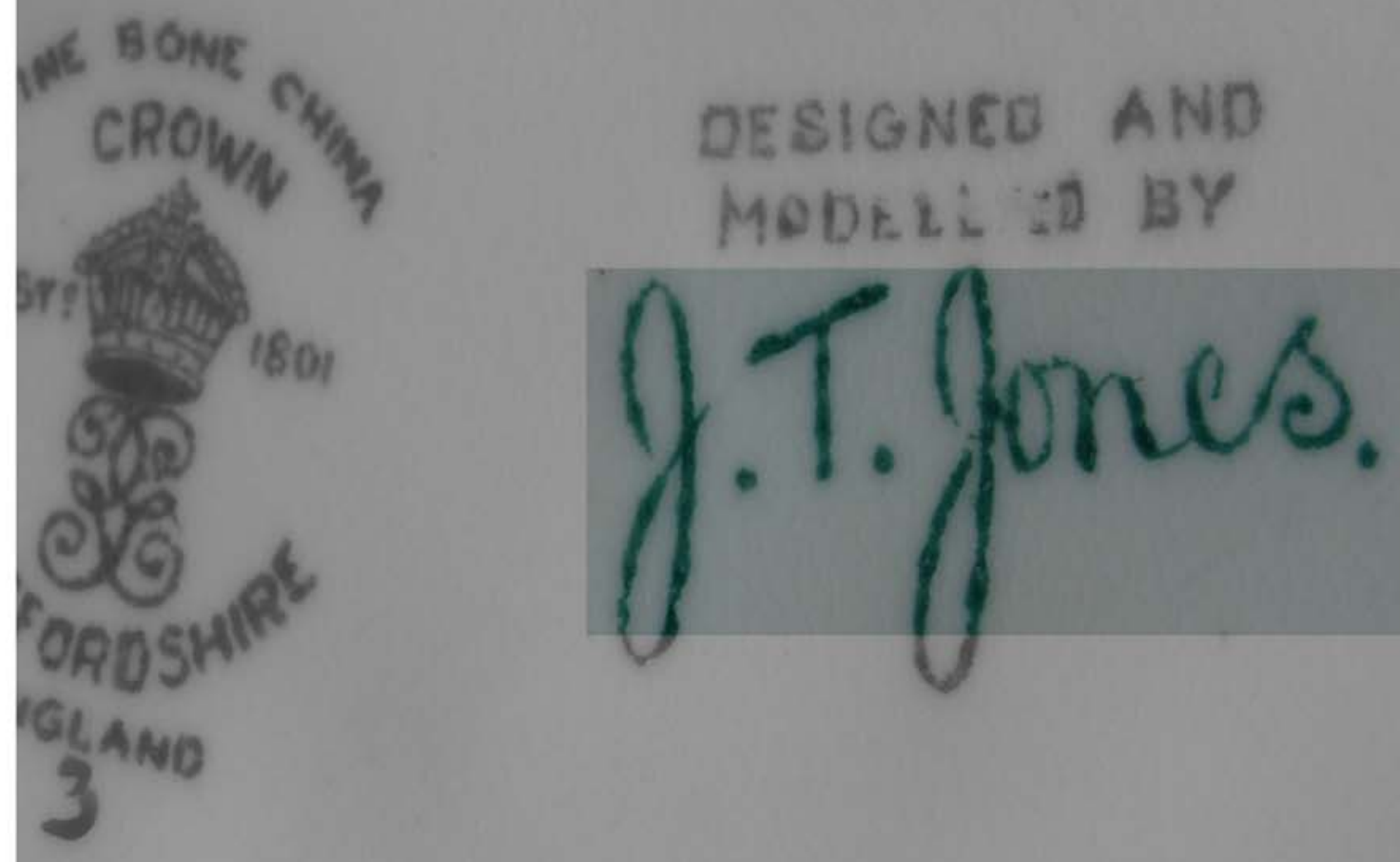

The designer and maker were acknowledged. 
The use of engraved initials defines ownership both physically and emotionally.

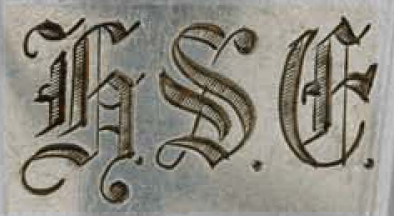


The punishment for falsifying a hallmark when it was introduced was death. 
The common practice of imitating materials has made products unknowable when new. 
This 21 st birthday present serves both a practical and ceremonial function. This image

was taken when the person was 22 .

It reveals the unsuitability of the materials and

the lost opportunity to create an heirloom. 


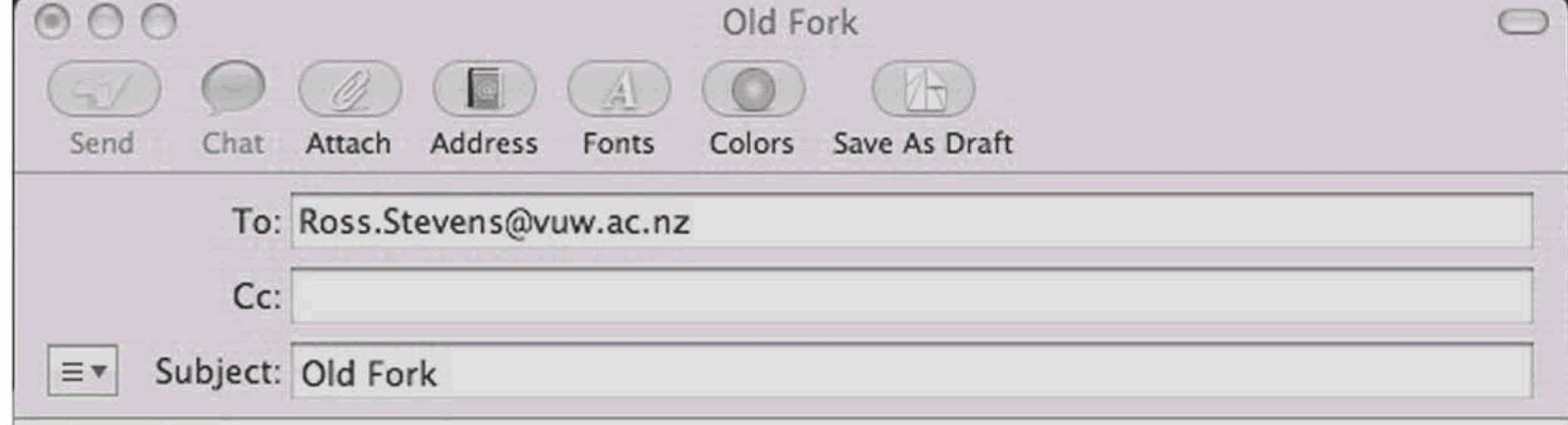

Hello Ross,

How are you?

I am in The Netherlands at the moment, looking for work and experiencing the culture.

I have attached a file of some photos that i thought you might like, there is a little story that goes with them.

Every night $i$ eat dinner with my Oma and every night $i$ have the pleasure of watching her eat with the same fork. First of all $i$ asked her "Oma why do you eat with the same fork every night?" Her reply was that when Manus (a relation and helpful hand with the Orchid, whom is now 100 years old) used to come and eat with family she decided that she wanted her own fork. I think part of her was afraid of sharing the same utensils with him. She also said that she eats well with the fork. Then I asked her a few nights later how long have you been using this fork for, and her reply was 40 years, Which i find just amazing... My Oma is now 83 years old and $i$ know that this fork is like a stable part of her day, infact $i$ just don't know if she could exsist the same without it.

So anyway the photos attached are of this fork, and i just thought that you Ross would be interested in seeing what 40 years + of eating with the same fork would look like. A great experiment, if you have the patience. 


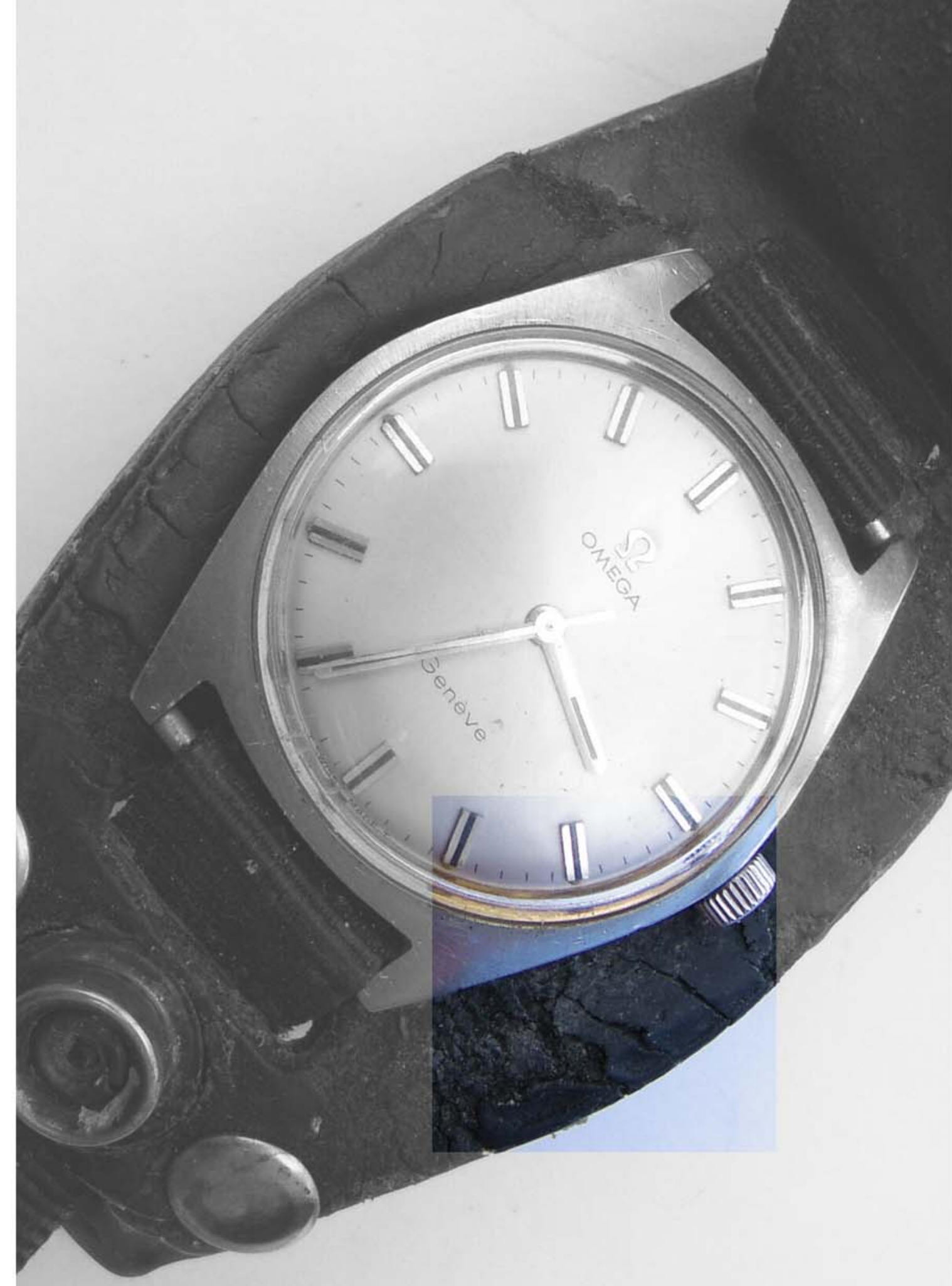

Through its forty year life this watch has continually shed its protective leather skins yet the stainless steel body has remained constant. 
Through use, damage and repair this pocket watch has transformed from a "Tissot Antimagnetique" into simply my "grandfathers" watch. 


$$
\text { L.M.STEW }
$$

The engraved name in the watch confirmed my grandfather as the author of the wear. 


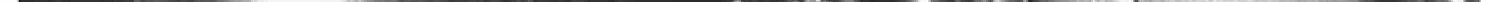


The damage in this product reveals careless use and proven durability.

\section{inis}

1. 50,270 s

mas .

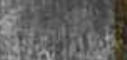

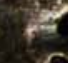

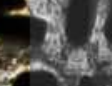

1

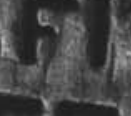

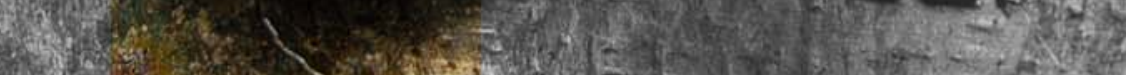

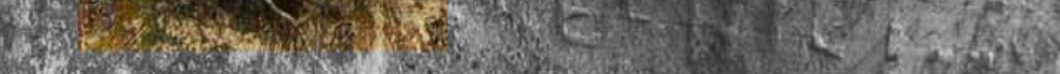

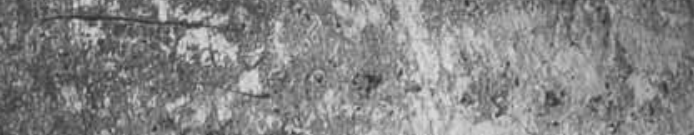

6. 1 .

Q

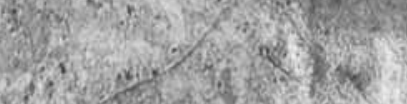

H.

2.0.

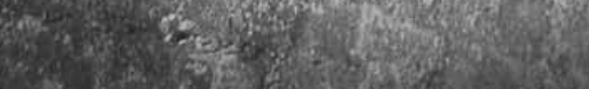

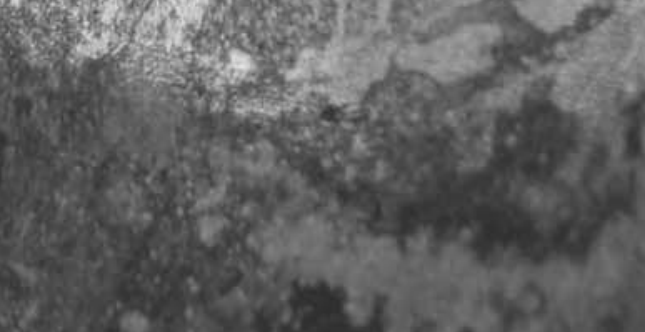




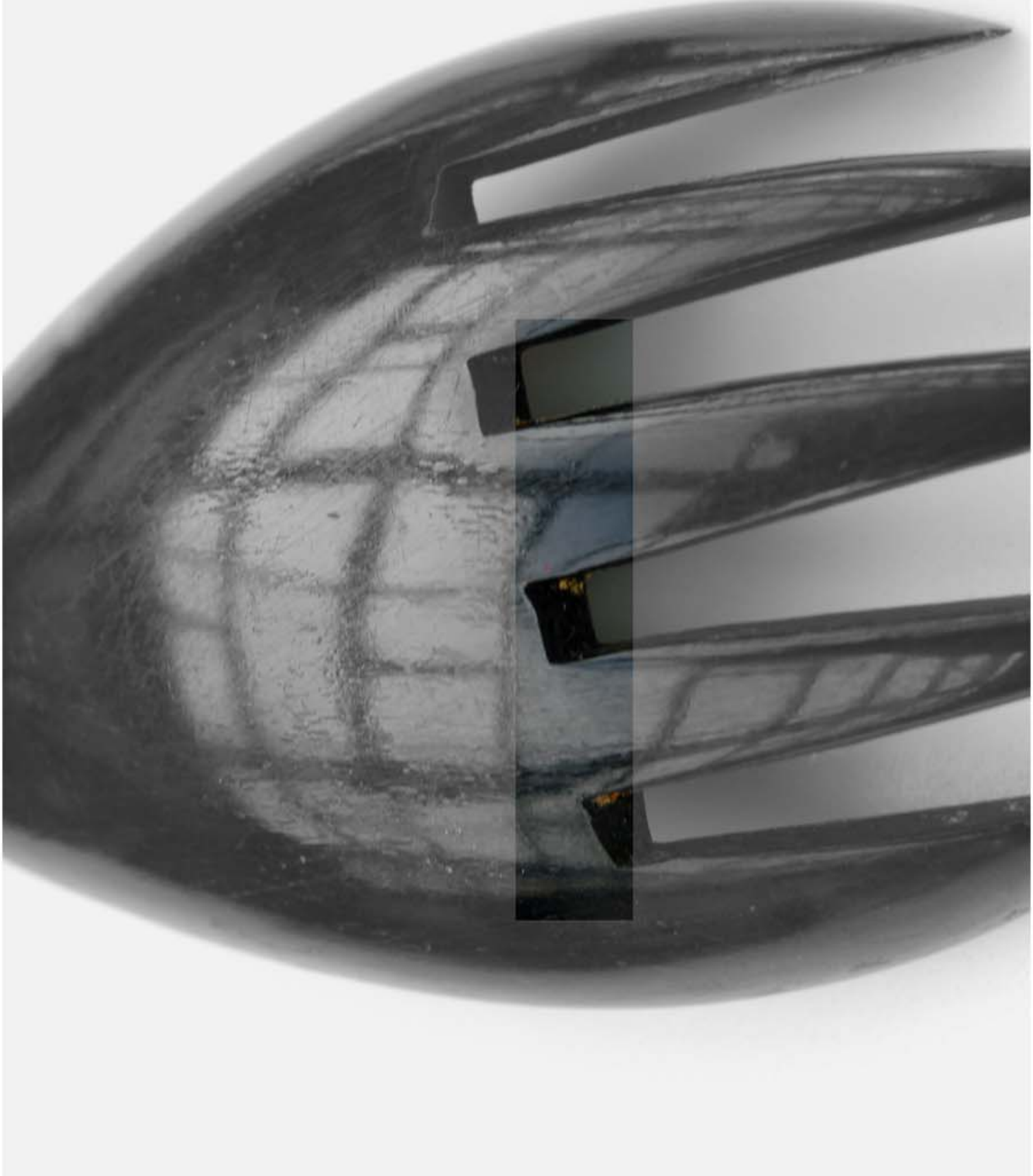

Variation is one of the valued qualities of handmade product. 


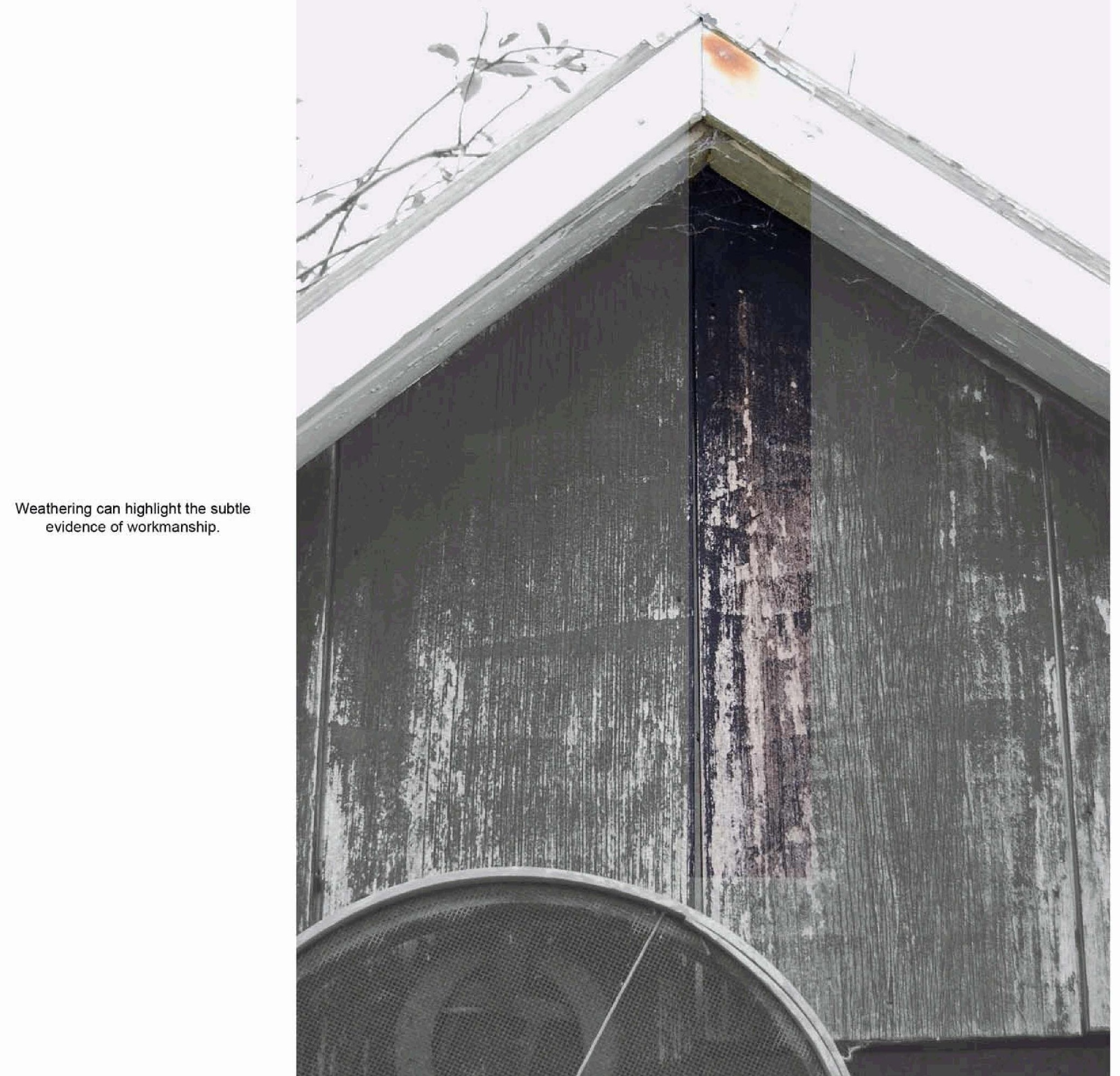


Wear can hightlight manufacturing variations. 
The form of the product can focus or diminish wear. 
Orientation and balance can influence wear patterns. 
Wear intensity reveals the frequency of use (level 1 of building)

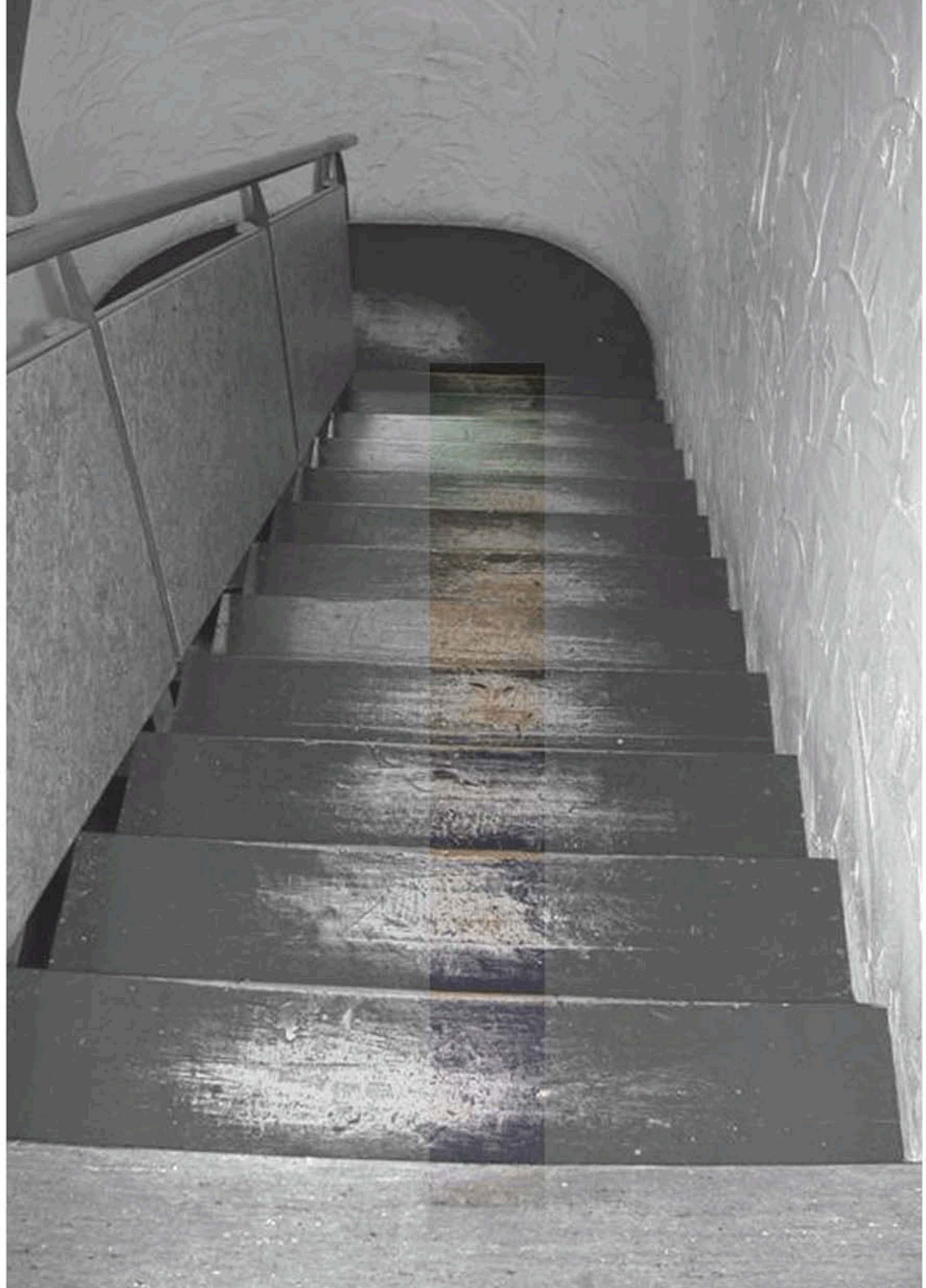


The primary tool used to create wear

patterns in portable electronic

products is the human hand. 
The diversity of the human hand is transferred and evidenced in the product. 
The interaction between the body and the product is evidence through wear patterns.

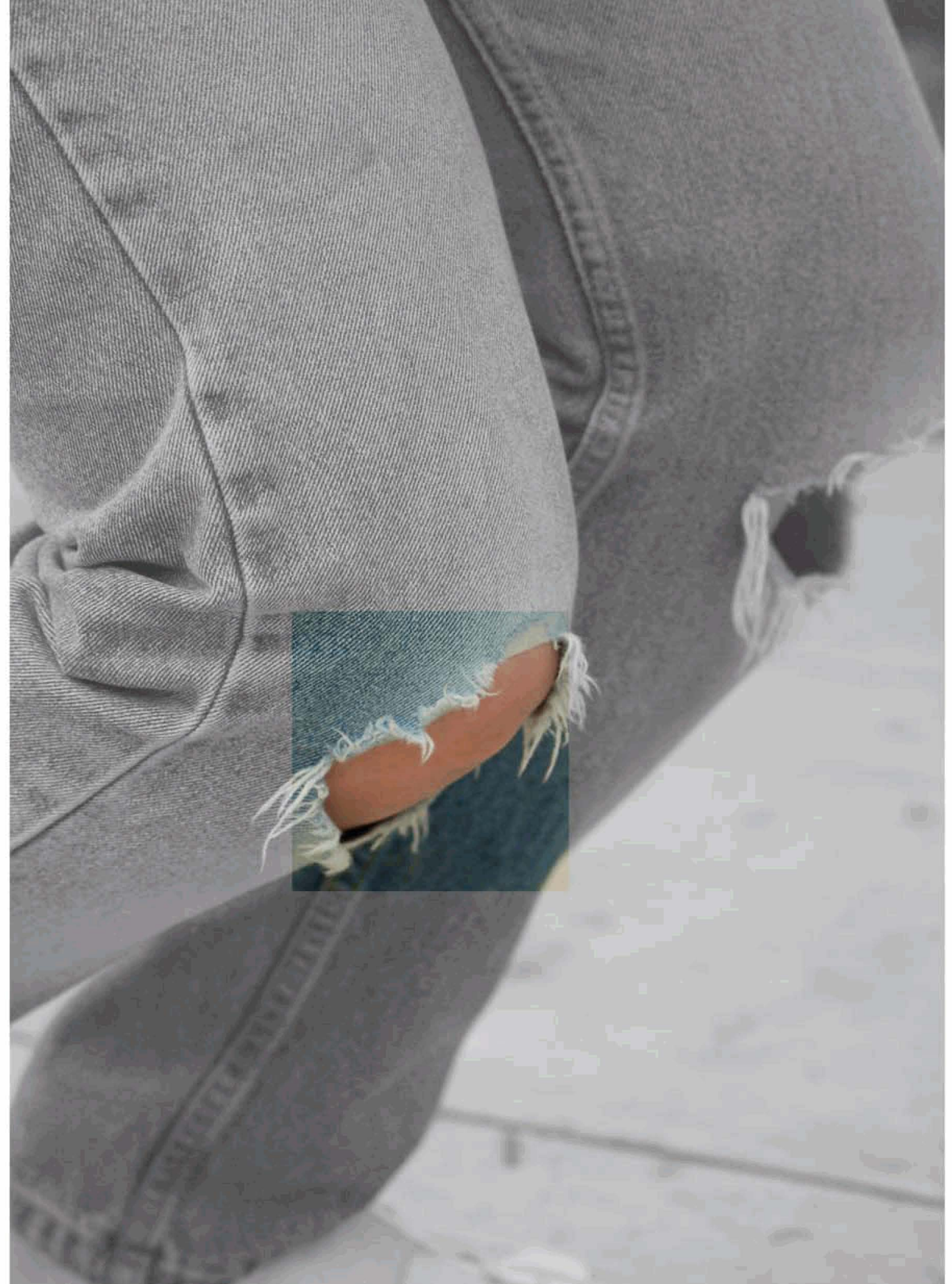


Corresponding wear occurs in the body that is concealed through continual growth. 

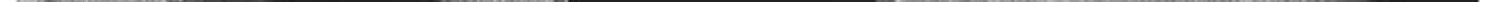

Wear has shaped the product to fit the body. 
Contrast between exterior and interior colours creates highly visible wear patterns. These patterns reveal soft continual erosion and hard abrupt damage.

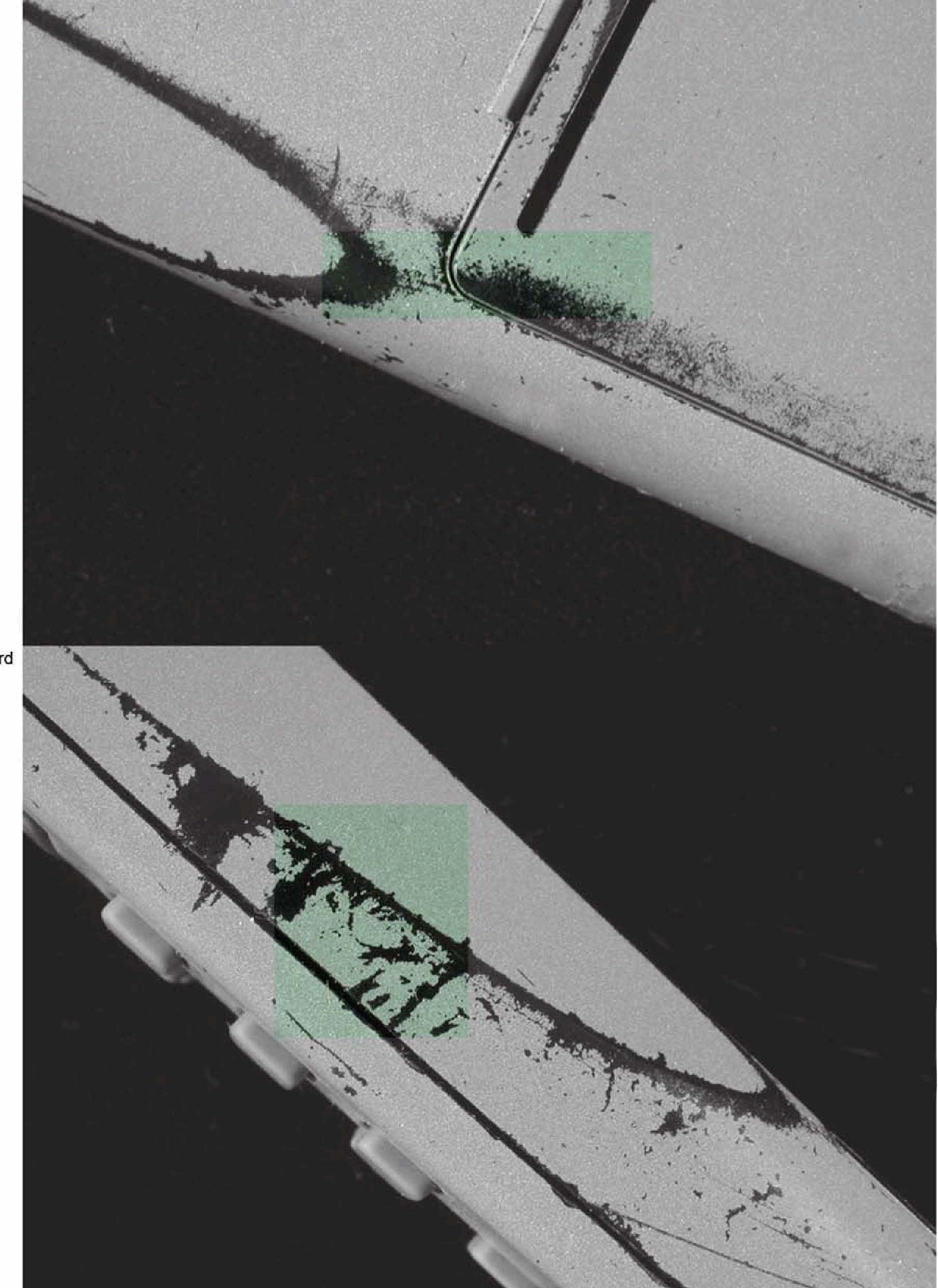




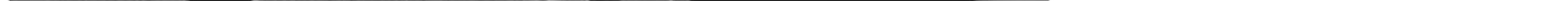


Variation to generic products that occur through use create a person specific fit and history. 
Complex reactions occur between materials.

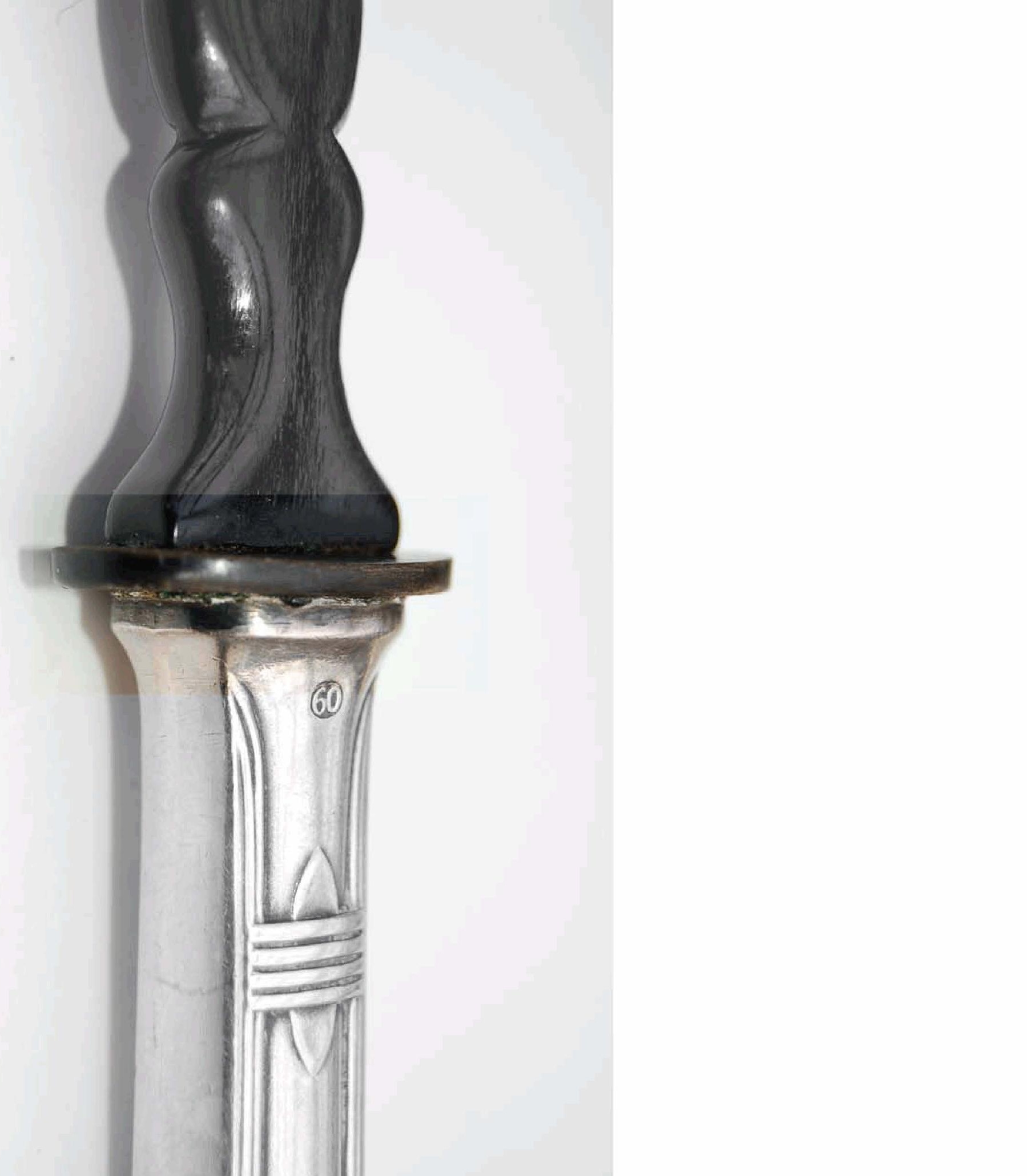


Weathering is a catalyst in creating reactions between materials. 
The reactivity of a material determines the speed of change. 
Two histories have been combined to create this scenario. The 60year old stirring wand was inherited from the grandmother of my wife and th drink recipe was created by my mother. 
Variations in the growing process have created a complex material. 


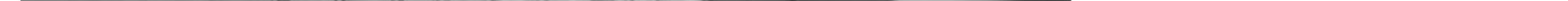



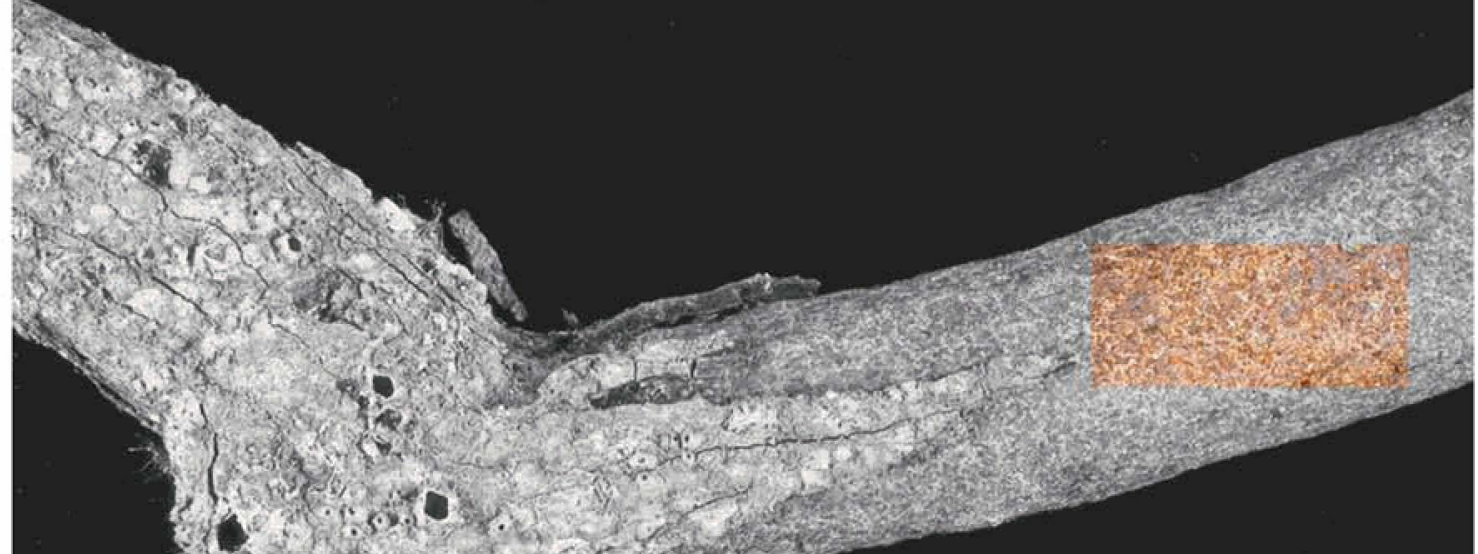

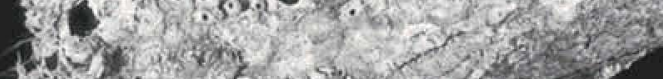

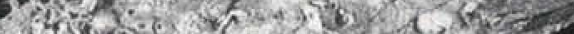

(1) 10

c. 32 : 12

ifis

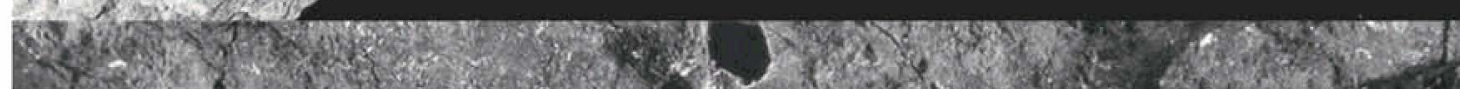

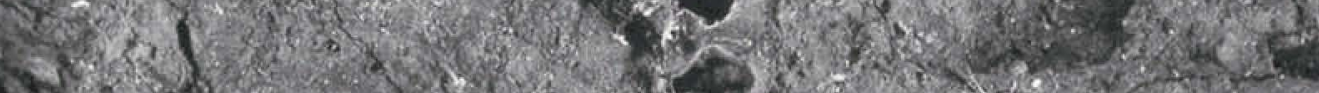

tis

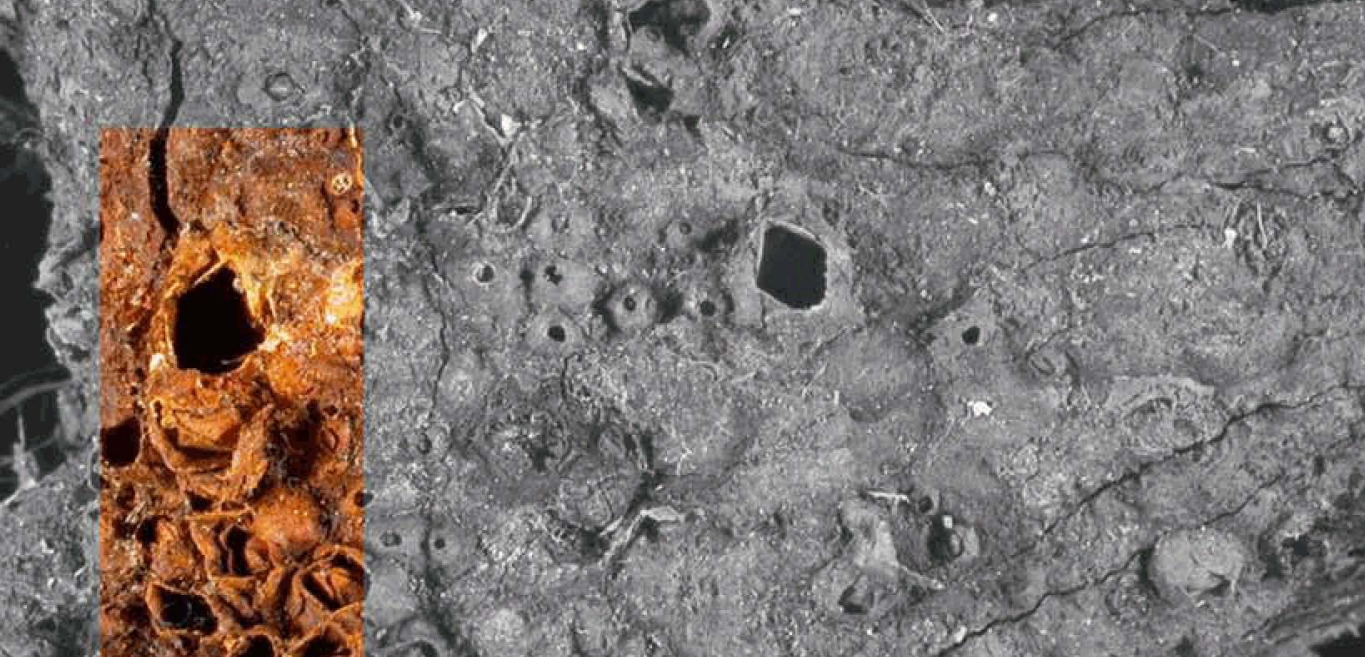

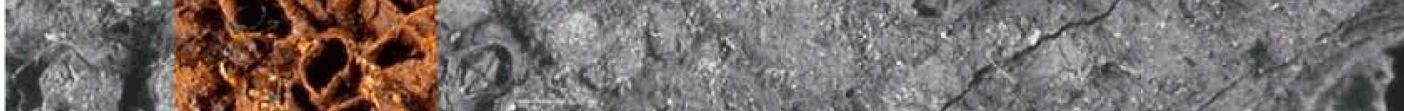

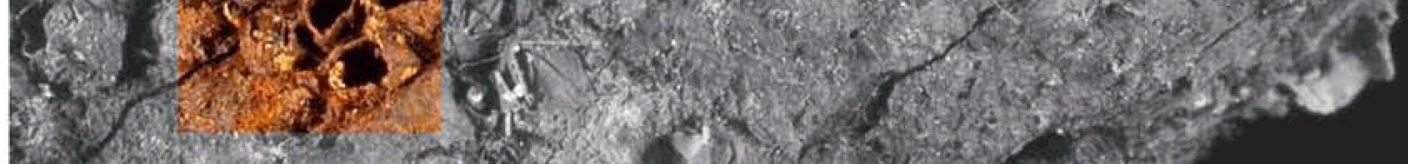

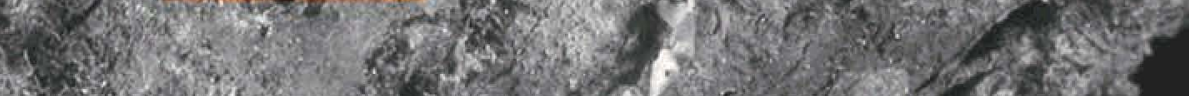

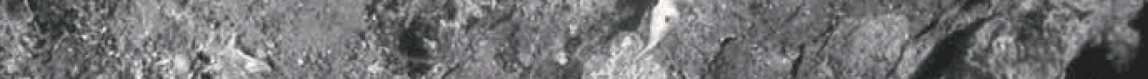




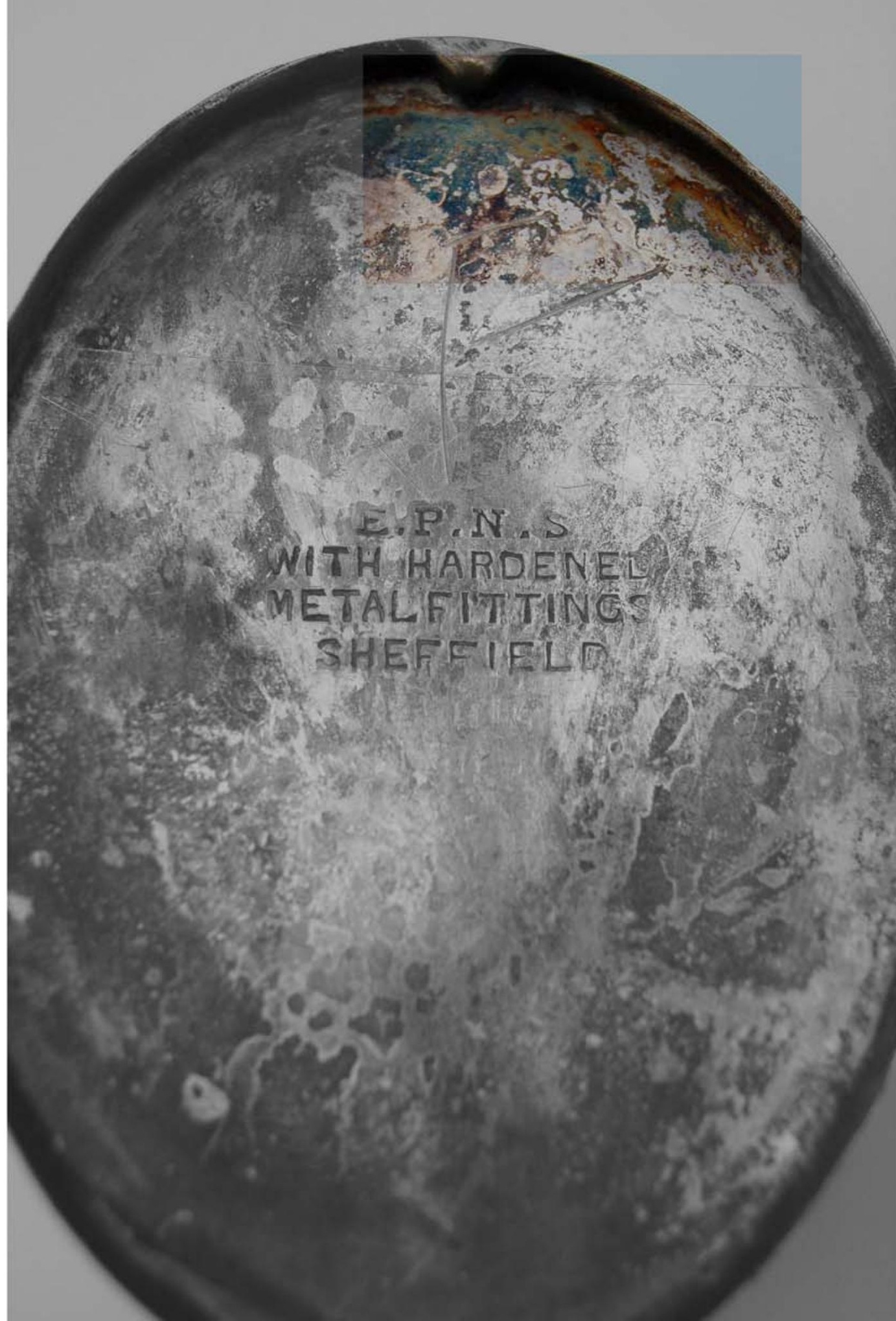

The fluidity of the oxidation process remains evident in the dry stain. 
The growing plant and the rusting stee interact to create a pattern expressive of the prevailing wind.

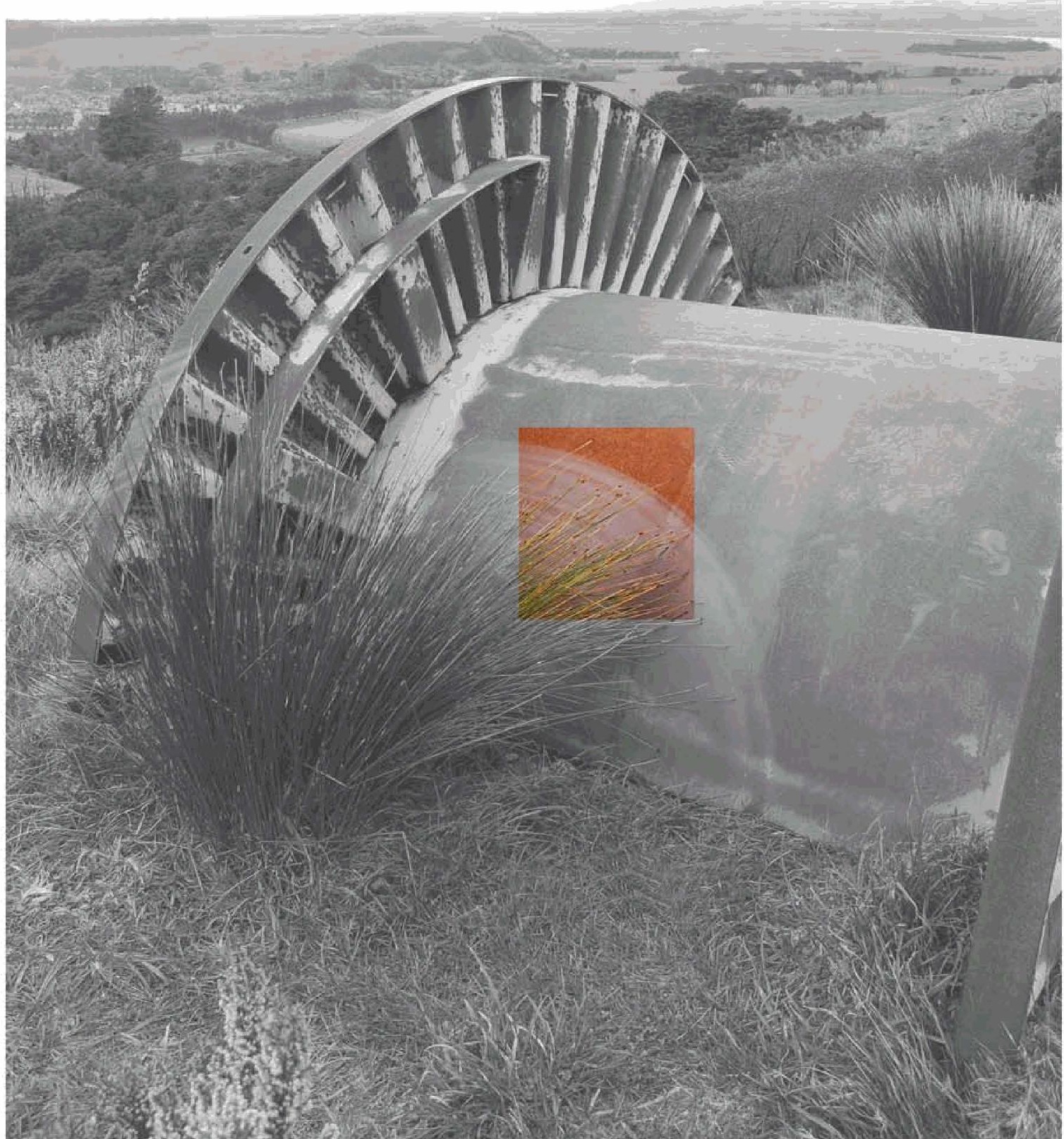




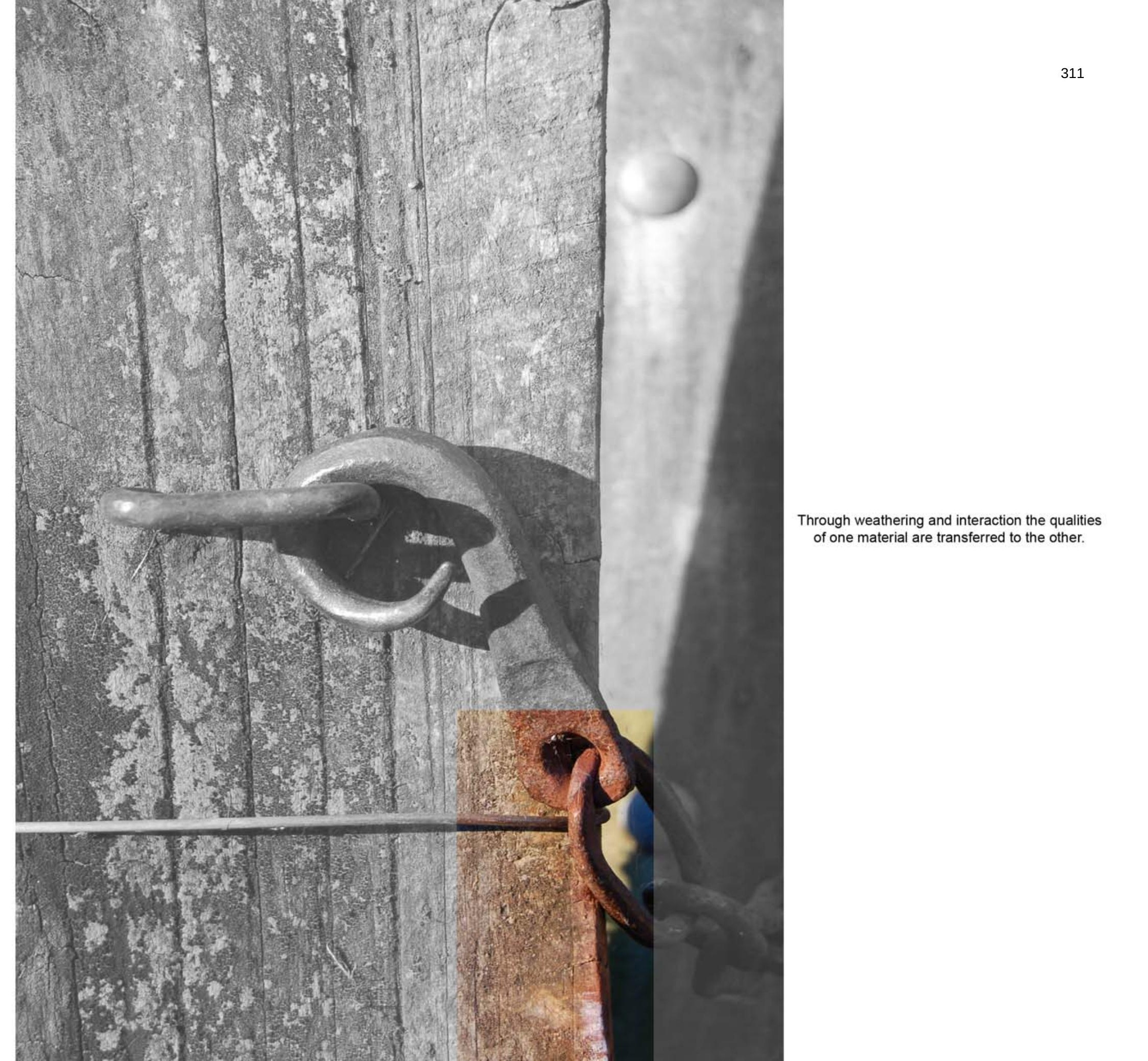




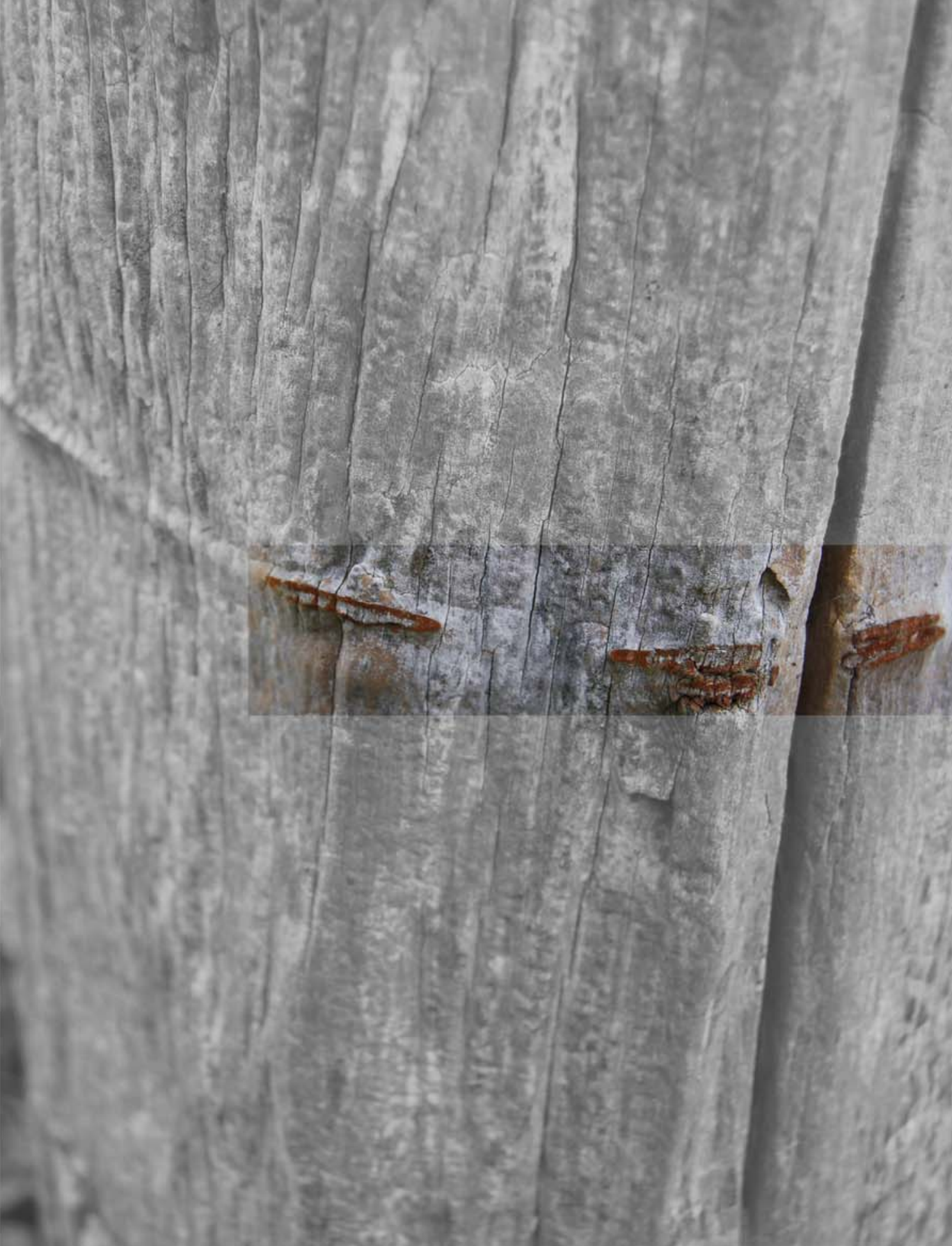

The previous existence of the wire is left evident in the weathered timber. 
The lines inherent in the 3D printing process become visible through wear. 
The basic function of the tea spoon has become secondary to a display of technical prowess.

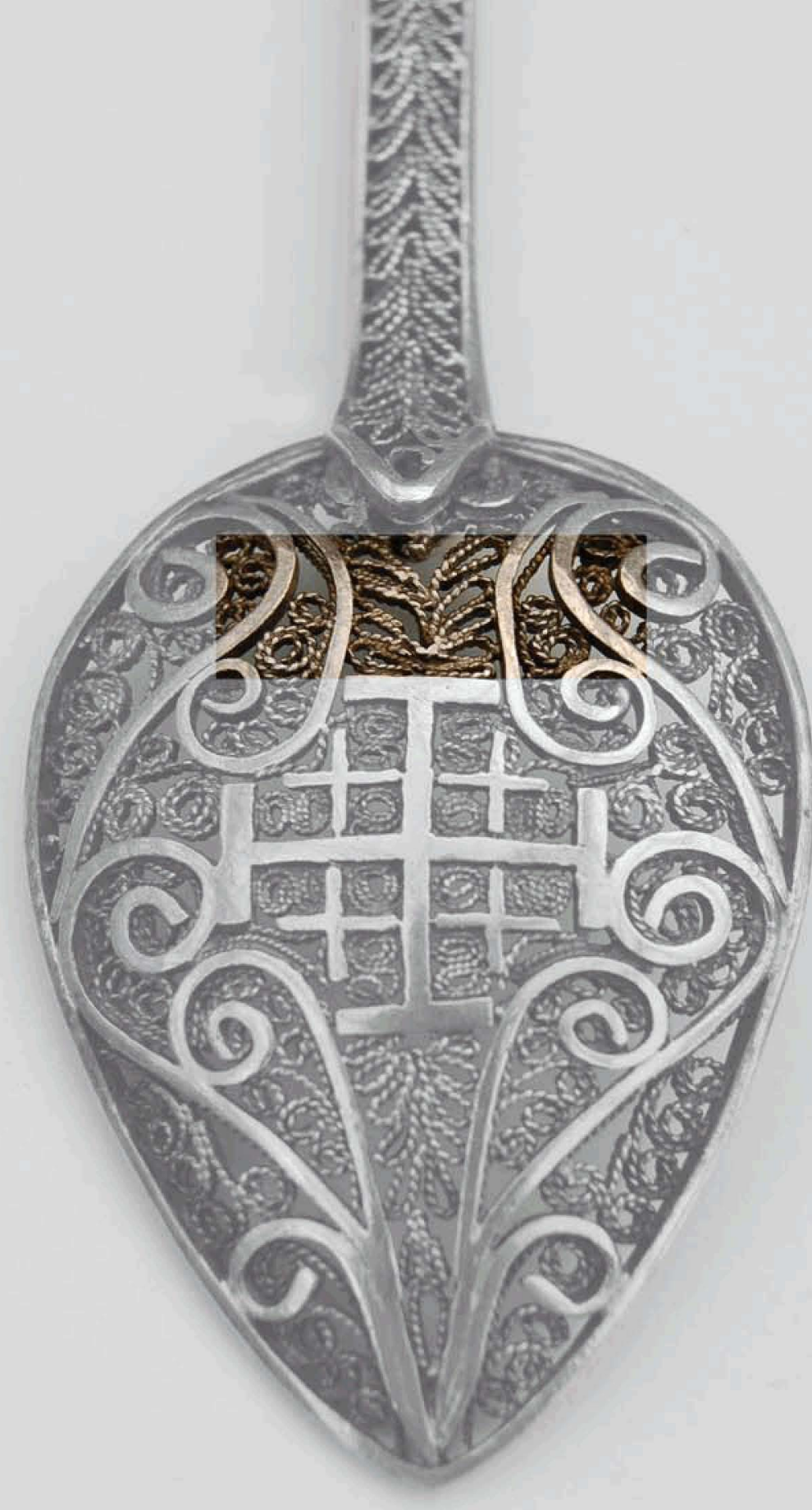




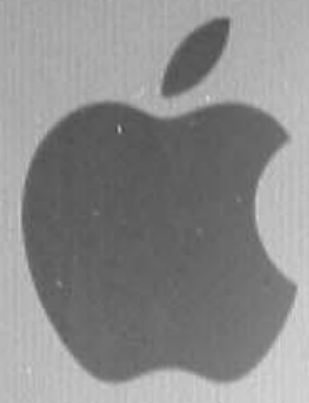

The technology with which we share our lives is empowering yet fragile.

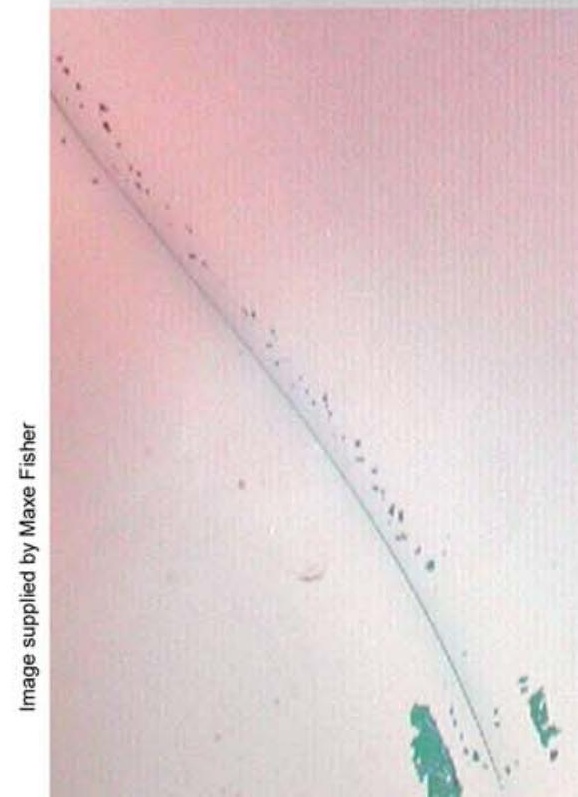



Bibliography 
Primary Reading

Garreau, Joel. (2004) Radical Evolution. New York, USA, Doubleday, pp53-65.

Hinte, Ed van. (2004) Eternally Yours: Time in Design. Rotterdam, Netherlands, 010 publishers, pp 198-213.

Hinte, Ed van. (1997) Eternally Yours: Vision on Produot Endurance. Rotterdam, Netherlands, 010 publishers, pp 101-119.

Lupton, Ellen., Miller J.Abbott. (1992) The Bathroom, The Kitchen, and The Aesthetics of waste. Cambridge, USA, MIT List Visual Arts Center, pp 2-9.

McDonough, William., Braungart, Michael. (2002) Cradle to Cradle. New York, USA, North Point Press, pp 27-66.

Mostafavi, Mohsen., Leatherbarrow, David, (1993) On Weathering. Cambridge, USA, The MIT Press, pp 80-108.

Pye, David. (1978) The Nature and Art of Workmanship. London, England, Cambridge University Press, pp 30-58.

Sterling, Bruce. (2005) Shaping Things. Cambridge, USA, The MIT Press, pp 37-57.

Taylor, Mark C. (1997) Hiding. Chicago, USA, The University of Chicago Press, pp 165-217.

Verbeek, Peter-Paul, (2005) What Things Do. Pennsylvania, USA, Pennsylvania State University Press, pp 135-166. 


\section{Secondary Reading}

Abram, Joseph. (2005) Pathfinders. Basel, Switzerland, Birkhauser, pp12-35.

Adamson, Glenn. (2003) Industrial Strength Design. Cambridge, USA, The MIT PresS, pp195-208.

Callicott, Nick. (2001) Computer-Aided Manufacture in Architecture. Oxford, England, Architectural Press, pp 27-43.

Frens, Joep. (2006) Designing for rich interaction. Eindhoven, Netherlands, Technische Universiteit, pp14-22.

Gausa, Manuel. (1999) Spirals: open time, fractal time. Barcelona, Spain, Collegi d'Arquitectes de Catalunya, pp 38-49.

Koren, Leonard. (1997) Wabi - Sabi. Berkeley, USA, Stone Bridge Press, pp54-73.

Krieger, Martin H. (2000) What's wrong with plastic trees? Connecticut, USA: Praeger Publishers, pp124-130.

Lupton, Ellen. (2002) Skin : surface, substance, and design. New York, USA, Princeton Architectural Press, pp31-61.

Lynn, Greg., Rasid Hani (2002) Architectural Laboratories. Rotterdam, Netherlands, NAi Publishers, pp52-61.

Manzini, Ezio. (1989) The Material of Invention. Cambridge, USA, The MIT Press, pp28-58.

Margolin, Victor, (2002) The Politics of the Artificial. Chicago, USA, The University of Chicago Press, pp 28-37.

Massad Fredy., Guerrero, Alicia Yeste. (2002) a+a architecturanimation. Barcelona, Spain, Actar, pp300-341.

Molotch, Harvey. (2003) Where stuff comes from. New York, USA, Routledge, pp5-13.

Rahim, Ali. (2000) Architectural design: Contemporary Processes in Architecture. London, England, Wiley-Academy, pp26-35. 
Websites

Arthur, Charles. (2005) The Register, www.theregister.co.uk/2005/09/23/ipod_nano_scratching

Bennett, Mathew. (2006) British Chiropractic Association (BCA), www.theregister.co.uk/2006/02/22/text_injury

Carucci, Elinor. (2002) Closer, www.elinorcarucci.com

Center for Materials and Surface Analysis, www.latrobe.edu.au/surface/about.htmI

Clifford, Bronwyn. (2005) Two Thumbs Down, www.ergoweb.com/news/detail.cfm?id=1188

Eno, Brian. (1996) The Clock of the Long Now, www.longnow.org

Gershenfeld, Neil. (2003) The Personal Fabricator, www.kurzweilai.net/meme/frame.html?main=/articles/art0574.html?

Hekkert, Paul. (1999) The Design and Emotions Society, www.designandemotion.org

Morton, Chip. (2002) Post-Consumerism R, www.post-consumerism.com/post.htmI

Piller, Frank (2007) http://mass-customization.blogs.com/mass_customization_open_i/2007/01/the_next_custom.html

Sanford, Glen. (1996) www.apple-history.com

Van Hinte, Ed. (1996) www.eternally-yours.nl

Wood, Bethan. (2006) Stain, www.wood/ondon.co.uk/wood_pages/3rd year/stain_pages/stain3.html

(2006) www.vertu.com 